
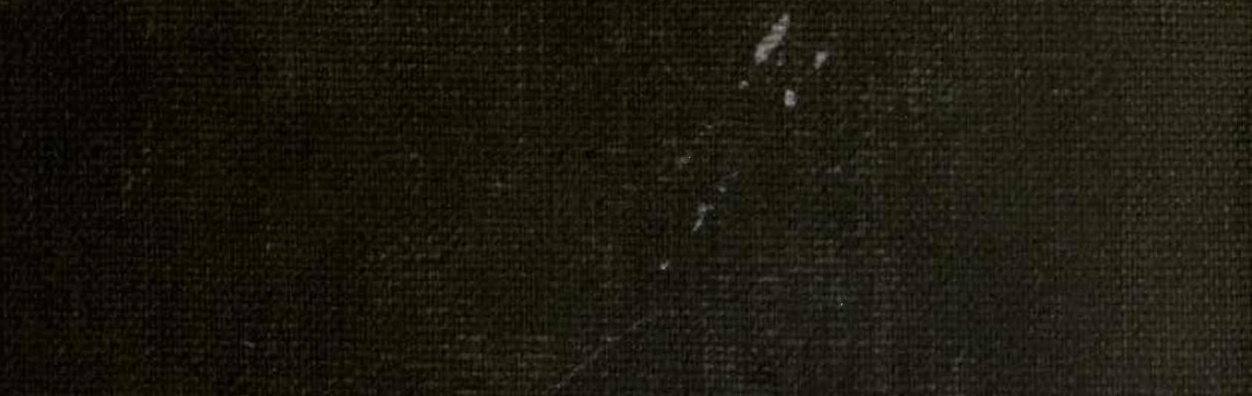


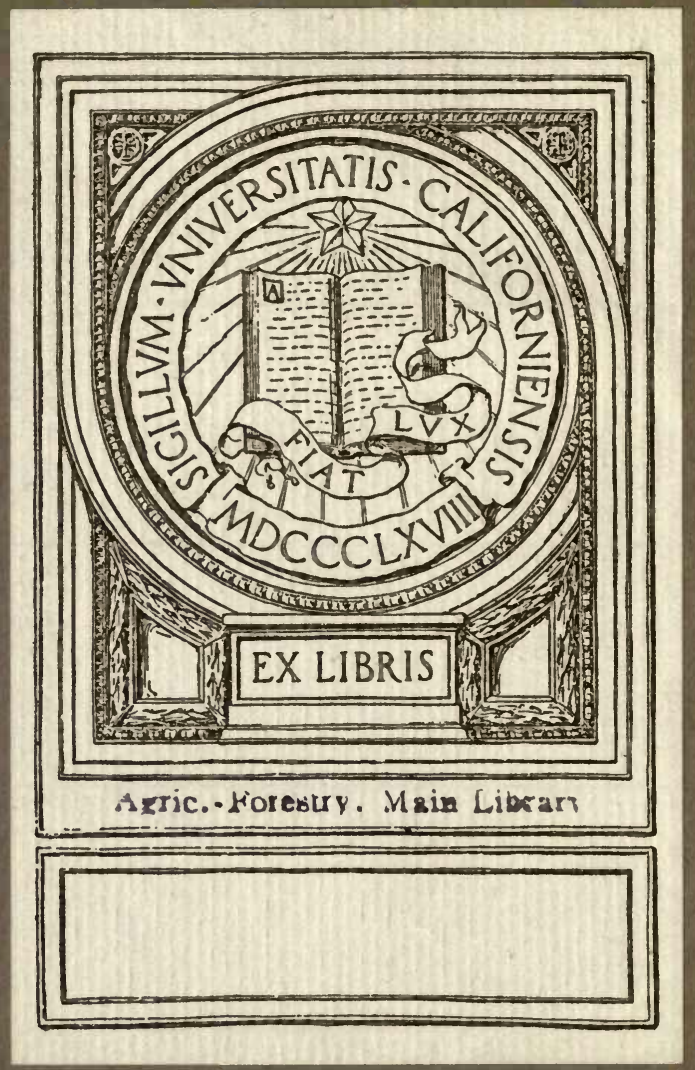





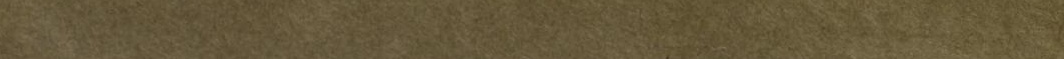

(3)

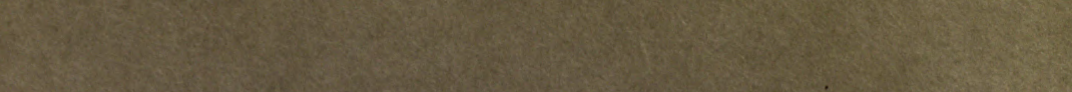

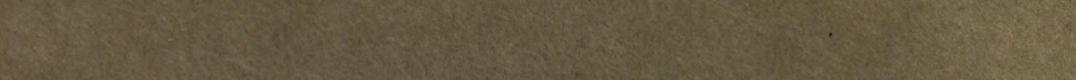

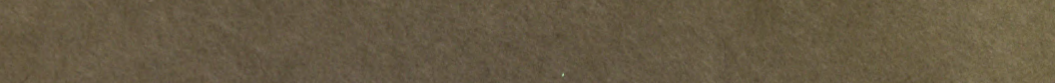

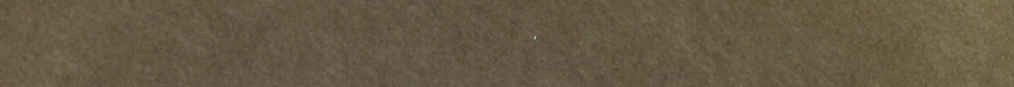

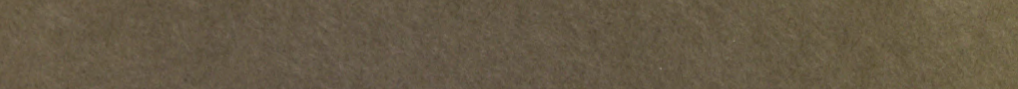

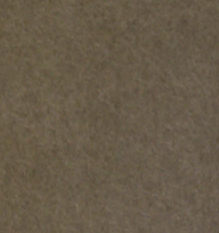
(3) (5)

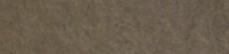

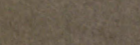
(4) (6) 5n: 20. 1.7. 5.20. m.6.

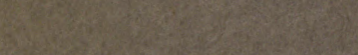
tis? W.

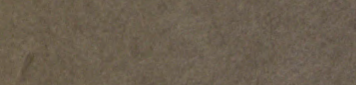

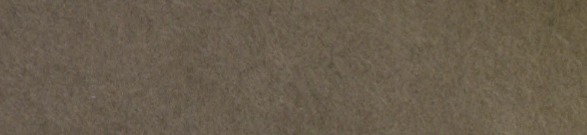
4. 25.

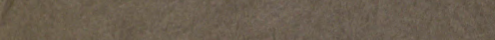

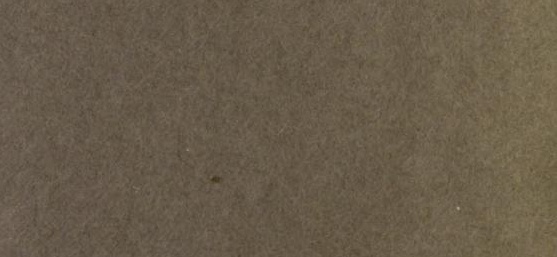

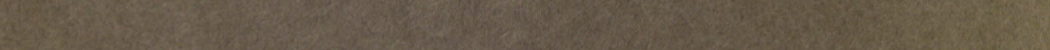
$6 x^{2} x^{2}+1$ 





\section{Direct Colour Photomicrograph.}

Screen-plate process (Autochrome).

Crystals of Salicin under Polarised Light $\times 18$

Objective ... ... Zeiss Planar $25 \mathrm{~mm}$. F. 4.5 .

Ocular ........ None.

Condenser ... Watson's UniversaI Achromatic, top lens removed. Polariser fitted under the condenser. A Selenite plate was also used.

Illuminant ... Liliput 4 amp. arc.

Aux. Condenser Double convex lens, projecting parallel light.

Screen $\quad \ldots \quad \ldots$ Lumière's screen for arc light.

Plate $\quad \ldots \quad \ldots$ Lumière's Autochrome.

Exposure ... ... 4 sec.

Developer $\quad$... Rodinal 1 in 10 , for $4 \mathrm{~min}$. 


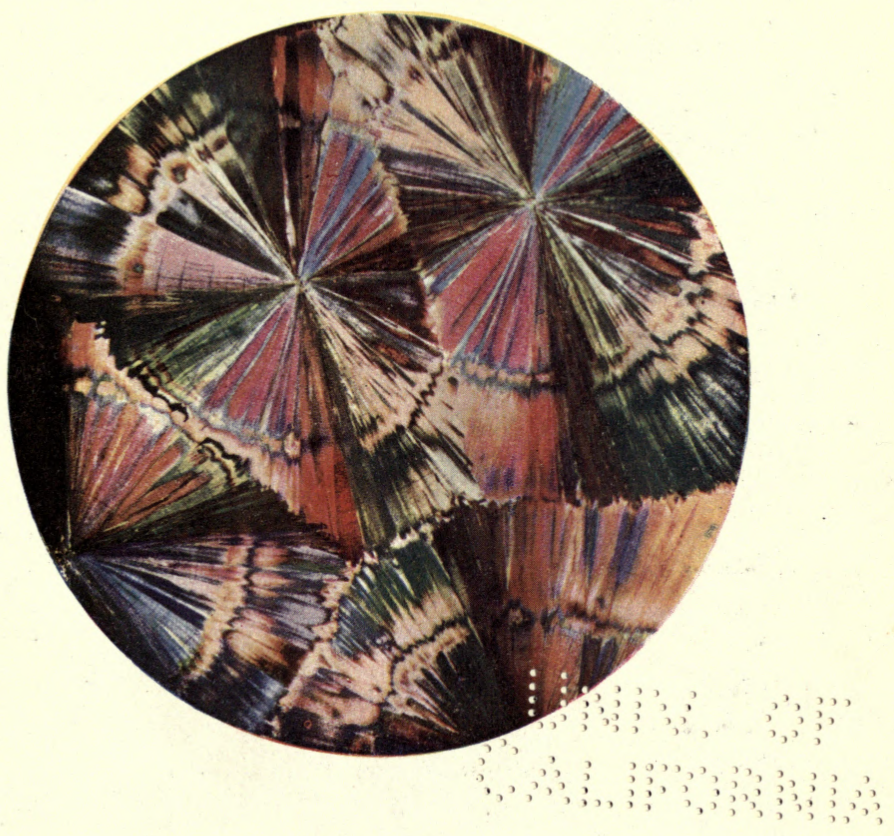


यद्य and 


\section{HANDB00K}

$\mathrm{OF}$

\section{PHOTOMICROGRAPHY}

BY

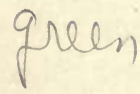

H. LLOYD HIND, B.Sc., F.I.C.

W. BROUGH RANDLES, B.Sc.

LECTURER IN BIOLOGY, DERBY TECHNICAI. COLIFGE:

With 44 plates, comprising 8 three-colour reproductions of direct colour-photomicrographs and 85 half-tone reproductions

of photomicrographs, and 71 text-illustrations

NEW YORK

E. P. DUTTON AND" CO.

LONDON : GEORGE ROUTLEDGE \& SONS, LTD. 


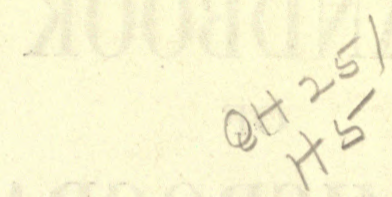

First published, December, 1913

Mum enchain

Agriculture 


\section{PREFACE}

THIS Handbook is the outcome of a series of articles originally published in the Photographic Monthly and intended as an introduction to photomicrography from a photographer's point of view. In this volume the subject has been approached from its two aspects, with descriptions of both photographic and microscopic technique, and it is hoped that experts in either field will look with indulgence on an elementary treatment of the subjects with which they are familiar. At the same time, however, the processes are discussed in sufficient detail to be of use in research, for which the full possibilities of the combination of microscopy and photography are scarcely yet realised.

In a handbook devoted to general processes and methods it is impossible to give an adequate account of the many uses of photomicrography in technology and industry, but an attempt has been made to indicate briefly some of its main applications. Many, perhaps equally important, have necessarily been omitted. The methods described may, however, be applied by any worker with the microscope to his own particular investigations.

In the preparation of some of the more specialised portions, for reading of proofs, and for valuable suggestions, the authors have to acknowledge the kind help of several friends, in particular Mr. E. Collier Green, Drs. N. S. Finzi 
and C. T. Holford, and Messrs. L. Archbutt, G. H. Broom, and R. Lyon. Their thanks are also due to manufacturers and their representatives for the loan of valuable apparatus and of blocks for many of the figures in the text.

Several of the illustrations are from photomicrographs of objects procured from slide-lending libraries. Access to slides which have been mounted by specialists is invaluable for practice in photomicrography and for the study of objects otherwise unobtainable, but the worker is strongly recommended to make his own preparations whenever possible. As a help in that direction the methods adopted in preparing some of the objects illustrated in the plates are described.

In conclusion we must recognise with thanks the great care and courtesy with which the Publishers have always met our wishes in regard to the illustrations and general arrangement of the book.

$$
\begin{aligned}
& \text { H. L. H. } \\
& \text { W. B. R. }
\end{aligned}
$$

November, 1913. 


\section{CONTENTS}

CHAPTER

PAGES

I. Photomicrographic Apparatus . . . . 1-24

Horizontal cameras - Vertical cameras - The workroom-Prevention of vibration.

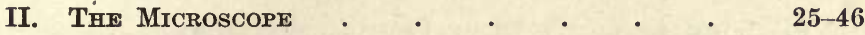

Essentials of a photomicrographic stand-Descriptions of typical microscopes.

III. ObJectives AND Eyepieces a . . . 47-82

Defects and correction of lenses-Types and properties of objectives-Eyepieces-Magnification.

IV. Lamps and Illuminants . . . . . . 83-101

Suitability of various radiants for photomicrography-Description of lamps-Selection of an illuminant.

V. Condensers . . . . . . . 102-113

Collecting lenses-Substage condensers.

VI. Experiments on Illumination * • • 114-126

Critical light-Experiments with condensers and microscope-Use of the auxiliary condenser.

VII. Low Power Photomicrography . . . 127-141

Photography of small solid objects-Transparent objects-Telephoto lenses.

VIII. Critical Photomicrography . . . 142-152

Preliminary examination of the object-Illumination without an auxiliary condenser-With collecting lens systems.

IX. Colour Screens and Colour Sensitive Plates 153-168

Light filters and their use-Colour sensitive plates.

X. Exposure . . . . . . . 169-175

Trial exposures and exposure records-Systematised exposure. 
CHAPTER

PAGES

XI. Oblique and Dark-Ground Illumination

Cinemato-micrography-Polarised light.

XII. Opaque Objects-Metallography .

Low powers-Stereoscopic photomicrographyMetallography.

XIII. Colour Photomicrography

203-213

Three-colour and two-colour processes-Screenplate processes.

XIV. Рhotographic Operations

Plates and development-Printing processes

-Enlarging - Lantern slides-Safelights.

XV. Some Applications of Photomicrography

Medical practice-Bacteriology-Brewing-Living micro-organisms-Solid objects-Insects-Diatoms - Plant and animal sections.

Appendix of Formule, Etc. .

$263-278$

Fixing reagents-Stains-Photographic formulæ Refractive indices-Weights and measures-Enlargement table.

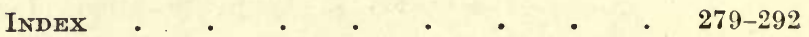

\section{LIST OF PLATES}

PLATE

1. Crystals of Salicin under polarised light $\times 18$

(Colour plate) Frontispiece

2. $\left\{\begin{array}{l}\text { A. Embryo Chick in Shell } \times 1 \\ \text { B, C. Fossils } \times 5 \frac{1}{2}\end{array}\right\}$

TO FOLLOW PAGE

3. Eye in Tail-feather of Peacock $\times 12$ (Colour plate) . . 32

4. $\left\{\begin{array}{l}\text { A. Group of Marine Shells } \times 8 \\ \text { B. Young Colony of Mould on gelatine plate } \times 25\end{array}\right\}$

5. Flower-bud of Salsify $\times 9$. . . . . . 48

6. Petiole of Cycas revoluta $\times 10$. . . . 48

7. $\left\{\begin{array}{l}\text { A. Section of Human Skin } \times 12 \quad \text { B. Cylindrospermum macrospermum } \times 250 \\ \text { B. }\end{array}\right\} \quad$. 64

8. $\left\{\begin{array}{l}\text { A. Proboscis of Blowfly } \times 15 \\ \text { B. }\end{array}\right\} \quad . \quad$. 80 
PLATE

9. $\{$ A. Proboscis of Blowfly $\times 105\} \quad 80$

10. Quartz Felsite under Polarised light $\times 10$ (Colour plate) . 96

11. Embryo Chick $\times 15$. . . . . . 112

12. Some faults commonly met with . . . . . . 120

13. Dog Flea $\times 40$. $\quad$. $\quad$. $\quad$. . . . 128

14. $\left\{\begin{array}{l}\text { A. Window Gnat } \times 7 \\ \text { B. Louse of } \operatorname{Cat} \times 70\end{array}\right\} \quad$. $\quad . \quad$. $\quad . \quad 132$

15. Longitudinal section of Mouse $\times 6 \frac{\pi}{2} \quad$. $\quad . \quad$. 136

16. $\left\{\begin{array}{l}\text { A. Section of Pitchstone } \times 60 \\ \text { B. Section of Lepidodendron selaginoides } \times 13\end{array}\right\} \quad$. 144

17. Control of contrast and definition by Colour Screens . $\quad 152$

18. $\left\{\begin{array}{l}\text { A. Hoof of Horse } \times 6 \\ \text { B. Section of Human Scalp } \times 45 \text { (Colour plate) }\end{array}\right\} \quad 160$

19. The same to illustrate the use of Colour Screens _. . 160

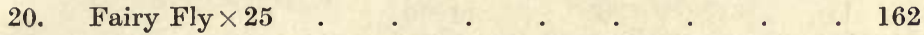

21. Comparison of Ordinary and Colour Sensitive Plates 168

22. Group of Diatoms $\times 56$ (Colour plate) . . . $\quad 176$

23. $\left\{\begin{array}{l}\text { A. Fairy Fly } \times 50 \\ \text { B. Group of Sponge Spicules } \times 50\end{array}\right\} \quad . \quad . \quad 176$

24. $\left\{\begin{array}{l}\text { A. Lophopus crystallinus } \times 20 \\ \text { B. Group of Polycistina } \times 45\end{array}\right\} \quad$. $\quad . \quad$. $\quad 182$

25. $\left\{\begin{array}{l}\text { A. Crystals of Cinchonidine } \times 45 \\ \text { B. Quartz diorite } \times 20\end{array}\right\} . \quad . \quad . \quad 188$

26. $\left\{\begin{array}{l}\text { A. Hind-leg of Bee } \times 16 \\ \text { B. Leaping Spider } \times 12\end{array}\right\} \quad$. $\quad . \quad . \quad . \quad . \quad 192$

27. Stereoscopic photomicrograph of Insect Eggs . _ 192

28. $\left\{\begin{array}{l}\text { A. Spiegeleisen } \times 45 \\ \text { B. }, \quad \times 500\end{array}\right\}$. . . . . 200

29. $\left\{\begin{array}{l}\text { A. Mild Steel } \times 50 \\ \text { B. Monel Metal } \times 150\end{array}\right\}$. $\quad$ • . . . $\quad 202$

30. Section of Head of Mouse $\times 8$ (Colour plate) • • . 208

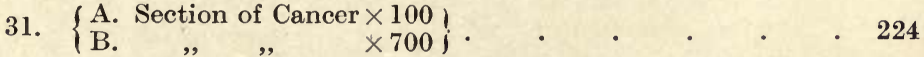
32. $\left\{\begin{array}{l}\text { A. Section of the same Cancer after Radium treatment } \times 100 \\ \text { B. , , , , , } \quad \times 700\end{array}\right\} 224$

(A. Bacillus anthracis with Spores $\times 1200$ )

$\left\{\begin{array}{l}\text { B. Kle "typhosus } \times 1200 \mid \\ \text { C. Klob̆ffler bacillus } \times 1200 \\ \text { D. Spirochota plicatilis } \times 600 \\ \text { E. Micrococcus in Pus } \times 1500\end{array}\right\}$ 
PLATE

TO FOLLOW PAGE

34. $\left\{\begin{array}{l}\text { A. Bacteria from an infusion of Peas } \times 1250 \\ \text { B. Bacillus tuberculosis } \times 1250\end{array}\right\} . \quad . \quad . \quad 240$

35. $\{$ A. Malaria parasite $\times 1200.2480\}$.

36. $\left\{\begin{array}{lll}\text { A. } & \text { Unstained micrococci } \times 1000 \\ \text { B. } & \text { C. } & \text { Yeast } \times 700 \\ \text { D. } & \text { Spirochoeta pallida } \times 1000, \text { dark ground } \\ \text { E. } & \text { ", Yeast } \times 700, \text { dark ground } & \text { Wild Yeast } \times 700\end{array}\right\}$

37. Culture Tubes (Colour plate) . . . . . 246

38. $\left\{\begin{array}{l}\text { A. Daphnia pulex } \times 20 \\ \text { B. Cyclops quadricornis } \times 18: \\ \text { C. Trypanosomes }\end{array}\right\} \quad . \quad . \quad 248$

39. $\left\{\begin{array}{l}\text { A. Arachnoidiscus ehrenberghii } \times 375 \\ \text { B. Orthoneis splendida } \times 620 .\end{array}\right\} . .256$

40. Triceratium favus $\times 620$. . . . . . . 256

41. $\left\{\begin{array}{l}\text { A. Actinoptychus heliopelta } \times 270 \\ \text { B. },, \quad \times 1250\end{array}\right.$

C. Pleurosigma angulatum $\times 720$

D. Navicula lyra $\times 1035$

42. Pleurosigma angulatum $\times 5000$. . . . . 256

43. $\left\{\begin{array}{l}\text { A. Surirella gemma } \times 1620 \\ \text { B. Amphipleurapellucida } \times 1250 \\ \text { C. }, \text {, } \times 3750\end{array}\right\} . .256$

44. $\left\{\begin{array}{l}\text { A. Root apex of Fritillaria } \times 420 \\ \text { B. Egg of Ascaris } \times 1200 .\end{array}\right\} \quad$. . . . $\quad 262$ 


\section{LIST OF ILLUSTRATIONS IN THE TEXT}

FIG.

PAGE

1. Camera with sliding carriers

2. Arrangement for enlarging camera . .

3. Watson's Laboratory camera

4. Reflex vertical camera .

5. Vertical camera . . 16

6. Bausch and Lomb's vertical and horizontal camera.

7. Leitz' large photomicrographic camera .

8. Mr. Barnard's geometric slide photomicrographic apparatus

9. Simple box camera

10. Simple vertical camera for instantaneous exposures

11. Sliding objective changer

12. Premier microscope

13. Circuit stage Van Heurck

14. Spencer microscope No.10

15. Zeiss' microscope No. 1 .

16. Leitz' microscope A

17. R.M.S. microscope .

18. Edinburgh Students' microscope $\mathrm{H}$

19. Diagram, Spherical aberration . . .

20. Diagram, Chromatic aberration
FIG.

PAGE:

21. Comparison of oil immersion and dry systems .

22. Mechanical and Optical tube lengths . . 71

23. Projection eyepiece $\quad 73$

24. Zeiss' 5-ampère automatic arc lamp . $\quad 87$

25. Enclosed arc lamp . $\quad 90$

26. Zeiss' Nernst lamp . . 91

27. The Hageh mercury vapour lamp . . $\quad 93$

28. Injector jet . . . 96

29. Incandescent gas lamp . 97

30. Oil lamp with adjustable condenser and screens

31. Bull's-eye condenser with iris on adjustable stand 104

32. Nelson's aplanatic condenser . . . 106

33. Abbe condenser $\quad . \quad 107$

34. Low power illuminator by Swift . . 108

35. Achromatic condenser, Watson's Universal . 108

36. Aplanatic oil immersion condenser by Leitz . 109

37. Swift's apochromatic condenser . . .

38. Diagram of illumination with auxiliary con denser .

39. Diagram of illumination by means of two auxiliary condensers .

125 
FIG.

PAGE | FIG.

PAGE

40. Diagram of reflecting illuminator

41. Camera for photography of insects . . .

42. Vertical camera for solid objects . . . . 131

43. Illumination of transparent object . . . 133

44. Zeiss' vertical and horizontal camera arranged for use with planars . 135

45. Diagram of illumination for small anastigmats . 136

46. Photomicrographic apparatus arranged for use with small anastigmats

47. Illumination by a parallel beam of light . . 149

57. Nelson's dark-ground condenser . . . 183

58. Diagram of illumination by Watson's paraboloid . . . .

59. Course of rays in Leitz' dark-ground illuminator.

60. Ernemann's cinemato micrographic appara tus . . . 188

61. Objective stop for stereoscopic photomicrography

62. Vertical illuminator 195

63. Prism vertical illuminator 196

64. The Rosenhain metallurgical microscope . $\quad 198$

48. Zeiss' large camera and Optical bench . . 151

49. Troughs for light filters . 160

50. Stops for oblique illumination . . . 177

51. Resolution by oblique light . . 178

52. Wheel stop . . 180

53. Traviss' expanding stop . 180

54. Diagram of dark-ground illumination by central stop . . . 181

55. Davis' diaphragm . . 181

56. Leitz' dark-ground illuminator

65. Arrangement of camera for micro-metallogra phy . . . .

66. Prism attachment for

- vertical illuminator . 201

67. Metallographic apparatus with inverted microscope . . . 202

68. Halation . . . . 218

69. Optical bench arranged for enlarging and projection . . . 231

70. Simple enlarging apparatus . . . 232

71. Apparatus for living organisms . . 248 


\title{
HANDBOOK OF PHOTOMICROGRAPHY
}

\author{
CHAPTER I \\ PHOTOMICROGRAPHIC APPARATUS
}

A PHotomicrograpHic outfit comprices a camera, microscope, and illuminating system, carried on a suitable baseboard or table. Each item is more or less elaborately fitted up with accessories, but the great majority of workers will find that quite a simple apparatus is adequate, and it may be said at the outset that the refinements in optical and mechanical details, which so rapidly run up the cost, are made primarily to facilitate difficult or high-power work, and to enable the most delicate adjustments to be carried out with rapidity and certainty. Difficult photomicrography can indeed be done with the simplest apparatus, provided the lenses are good, but the calls then made upon the skill and patience of the worker are much greater than when the adjustments are more or less automatic.

An ordinary stand camera, a student's microscope, and an oil or incandescent gas lamp, with a bull's-eye and Abbe condenser, low and medium-power objectives, and an eyepiece, will do perfectly well for many purposes, and very few workers require anything more elaborate. But the photography of diatoms requiring very high resolution, or of bacteria necessitating magnifications of 
"a thousand diameters or more, calls for lenses of high power ond igreat perfection, and a microscope and camera with correspondingly delicate movements.

Photomicrography should not be regarded merely as a method of recording observations, but also as a valuable means of research. Its evidence must therefore be unimpeachable, and this can only be the case when the chances of false images, due to defects in the optical parts of the apparatus or to their improper use, have been eliminated as far as possible. The optical equipment is consequently the most important part of the outfit, and the aim of the designer and maker of the microscope stand, the camera, and optical bench, is to provide a framework which will carry the lenses, and to fit it with mechanical adjustments of sufficient refinement to bring them to bear on the object, and allow them to work to their best advantage. This does not involve the use of the most expensive objectives and condensers when slightly less accurately corrected, and consequently considerably cheaper ones, will serve, as they undoubtedly will in the majority of cases; but it does mean that the accuracy of the fittings and the delicacy of the movements which carry and control the objectives, etc., must be commensurate with the corrections of the lenses, and that the latter be suitable for the work contemplated.

Although this view of the value of photomicrography is not lost sight of in what follows, the needs of the worker with restricted capital to outlay also have consideration. An endeavour is made to show how a considerable part of the apparatus can be made or adapted, and in the descriptions of camera, microscope, and illuminating system, the items which are luxuries, or refinements that may usually be dispensed with, are differentiated from those that are absolutely necessary.

Horizontal Cameras.-The Baseboard or Optical Bench.-There are two ways made use of for setting 
up the apparatus for photomicrography: the Horizontal, in which the optical axis is parallel with the table, and the Vertical, in which the camera is supported above the microscope. Preference is generally given to the former, particularly if a rather complicated illuminating system is employed. But many workers prefer the vertical arrangement, which has the great advantage that, with a simple lamp and condenser, an optical bench is unnecessary, as correct adjustment of the illumination can be carried out by means of the mirror. It is also more portable and compact, and with preparations in liquids it is generally the only practicable method to use. Each method has its advantages, and if a camera has to be adapted the choice may depend on its greater suitability for one or the other system.

No doubt the most universally serviceable stands are those made for both horizontal and vertical work, but caution must be exercised to make sure that no loss of stability is involved in the arrangement, and every possibility of vibration of the camera should be avoided.

A photomicrograph may be taken after no further preparation than is involved in placing the camera, microscope, and lamp in a straight line on a table, but the difficulties that have to be faced when using such an apparatus for anything but the lowest powers are at once apparent. Movement of the camera is almost inseparable from the action of drawing the slide, and renders accurate focussing of no avail. The readiest way to prevent this disturbance is to clamp the camera to the table; but other difficulties connected with the proper centration of the various lenses are also encountered, and as a rule it takes much longer to get the apparatus properly together and the illumination adjusted than to take the photograph and develop it. When changes of magnification involving alteration of camera extension have to be made, there 
will be further loss of time, as either the camera or microscope, or possibly both of them, will have to be unclamped and fixed in another position. These difficulties and others may be so easily overcome by the provision of a baseboard or optical bench of some kind that no attempt should be made to take photomicrographs without one, and a baseboard is so easily made by anyone having ordinary proficiency in the use of tools that the first thing to consider, after the camera, microscope, and illuminating system are obtained, is how to design one suitable for the apparatus. It must be constructed so that the optical axes of the microscope, lamp, and condensers shall be truly in line and perpendicular to the focussing screen of the camera, and so that any of the pieces of apparatus can be placed in its exact position without loss of time. Above all, it must be so rigid as to prevent vibration as far as possible in any part of the apparatus. Illustrations are given of arrangements we have made ourselves to adapt apparatus already possessed. They have proved adequate, and are offered primarily as an indication of the lines to be pursued. A different camera, microscope, or lamp may involve a different arrangement, but very little ingenuity is necessary to devise one suitable to the materials at hand.

Illustrations are also given of several cameras on the market, each of which is fitted with an accurately made optical bench. They are not necessarily any more efficient than home-made apparatus, though they may be expected to be much better finished, to work with greater nicety and precision, and all these points become of great importance in difficult work, and add immensely to the ease with which photomicrography is carried out.

The Camera.-A camera specially made for photomicrography can hardly be described as a necessity, and many workers are content to adapt the one they use for other purposes to microscopical work, or else to fit 
up a studio or field camera, and reserve it for photomicrography. Nor is there any need for discouragement when faced with these alternatives to a first-class professionally made outfit. If the camera fulfils the requirements set out below, it will answer the purpose admirably, and if set up in conjunction with the other apparatus with care and judgment, there is no reason at all why it should not serve for work of the very highest class.

In the first place, the base of the camera must lie perfectly flat on the table or board on which it is fixed, and the back must be capable of being adjusted so rigidly at right angles to this that the insertion of the dark slide, or the drawing of the shutter, causes no movement. The lens must be removable, as it is not used in photomicrography, and there must be a ground-glass focussing screen. In addition, a long extension will be found very advantageous, and if the camera can be extended from the back whilst the front remains in its original position, so much the better. A square bellows stand camera with a front of the same size as the back meets these requirements admirably, and it is easy to obtain an old-fashioned one without a lens for a very few shillings. A box camera fitted with focussing screen and dark slides can be used, and has the advantage of great rigidity; but the very great disadvantage that only one magnification can be obtained with one combination of objective and eyepiece, an objection that almost rules this kind of camera out for all-round work. A hand and stand camera of the type represented by the "Sanderson," "Vaido," or "Una" is perfectly suitable, but lighter types will be found rather trying to use. Reflex cameras are in certain cases adaptable, and sometimes, as for instance in the photography of living micro-organisms, are very useful. The apparatus illustrated in Fig. 4 includes a new ccmera, designed and patented by Mr. Lumley Cator, and marketed by Staley, called the "Convertible Britisher," 
which combines in itself the features of the last two groups of cameras. The reflex mechanism is contained in a separate segment that can be attached to the rest of the camera, or dispensed with at will, leaving an ordinary hand and stand camera.

The most generally useful size for the camera is half plate $\left(6 \frac{1}{2}{ }^{\prime \prime} \times 4_{4}^{3 \prime}\right)$, or its American or Continental equivalents, but quarter plate $\left(4_{4}^{\prime \prime} \times 3 \frac{1}{4}\right)$, or better, $5^{\prime \prime} \times 4^{\prime \prime}$, or $9 \times 12 \mathrm{~cm}$., is usually quite large enough. The $5^{\prime \prime} \times 4^{\prime \prime}$ has an advantage over the others in that its shape is better proportioned to include a circle without waste. Larger cameras than half plate are seldom required, but $10^{\prime \prime} \times 10^{\prime \prime}$ - or $10^{\prime \prime} \times 8^{\prime \prime}$ are often used on specially built outfits, and are very useful for enlarging purposes as well as photomicrography; the dark slides of the larger sizes should be fitted with adapters to take the smaller plates down to lantern-slide size $\left(3 \frac{1{ }^{\prime \prime}}{4} \times 3 \frac{1}{4}^{\prime \prime}\right)$ and $3 \frac{1}{2}^{\prime \prime} \times 2 \frac{1}{2}^{\prime \prime}$.

Adaptation of an Ordinary Camera.-Fig. 1 shows a method of fitting up an ordinary half plate camera. The baseboard is of well-seasoned wood $4^{\prime} \times 9^{\prime \prime} \times 2^{\prime \prime}$, with a groove $\frac{3}{16}$ " broad down its centre. Suitable blocks are cut to carry each component of the system, and strips of hard wood about $\frac{1}{2}$ " deep are let into them along their centre line; about $\frac{1}{4}$ " of these projects and slides in the baseboard groove. The blocks may warp if not of wellseasoned wood ; their thickness is very carefully adjusted so that the optical axes of the camera, microscope, and lamp may be in line. The microscope carrier is first made, and depressions cut in it to receive the foot of the microscope, and to hold the latter so that the tube lies truly along the centre line and perfectly horizontal. If the microscope will not stand steadily when the tube is horizontal, some means must be taken to support it. A clamp or wooden turn-button pivoted between the horseshoe serves very well, and if nothing better is at hand a temporary support may be made by placing 
a bottle and cork adjusted to the right height under the tube.

The optical axis of the microscope is then taken as the axis of the apparatus, and the block to carry the camera is planed until the centres of the lens aperture and ground glass fall in this line. The means adopted for clamping the camera to its carrier will necessarily be dependent on the form of the camera base. Sometimes screws or turn-buttons ean be used, in other cases a piece of wood may be placed over the baseboard and screwed to the block at either side to grip the camera tightly.

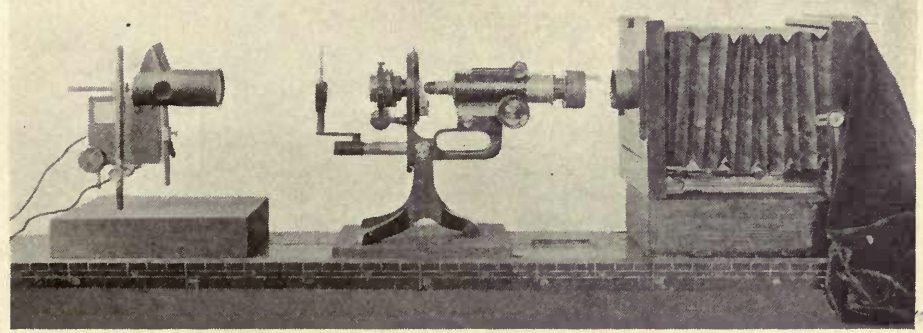

Firg. 1

CAMERA WITH SLIDING CARLIELS

It may be more convenient to provide two blocks for some cameras with long extension, the front and back being carried separately and sliding apart when the bellows are extended. If there is a centre panel three supports may be used.

Another block is adapted to the lamp. In the figure it will be noticed that this carries a rod on which the lamp slides up and down. The Liliput are lamp shown is fitted with a condenser, but in other cases it may be necessary to cut a further block for the condenser.

Arrangement for an Enlarging Camera.-The arrangement shown in Fig. 2 differs from the first mainly in 
the method used for sliding the carriers along the baseboard.

The camera is a large one $\left(10^{\prime \prime} \times 8^{\prime \prime}\right)$, with an extension of $26^{\prime \prime}$ and a breadth of $11^{\prime \prime}$, so that a baseboard $6^{\prime} \times 15^{\prime \prime}$ is used. A block to carry the camera is screwed on one end, and fitted with angle-irons at the front; the camera has a rigid base, so that it can be readily placed in exact position against these, and held there by a screw clamp at the back.

Two strips of wood about $3^{\prime}$ or $3^{\prime} 6^{\prime \prime}$ long, and $1^{\prime \prime}$ square section, are bevelled along one edge and screwed to the baseboard parallel to its length, and about 8 " apart,

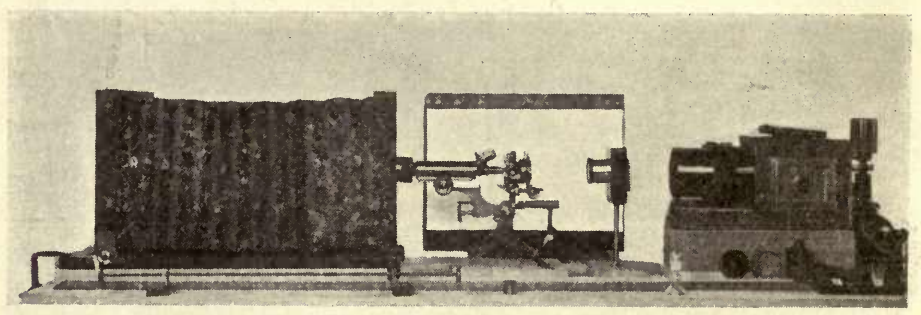

liig. 2

ARRANGEMENT FOR EXLARGING CAMERA

to form runners between which the carriers for the microscope and illuminating apparatus slide. The carriers are made of inch-board bevelled to dovetail into the strips, and on them the microscope, lamp, and condenser are fitted as in the first example.

An ordinary optical lantern with $4^{\prime \prime}$ condenser is shown in the figure as the source of light. A cooling trough for water or fluid light-filters is carried in the holder provided for lantern slides. A bull's-eye condenser is also shown, and can be used in conjunction with the large condenser, or in place of the latter; it is screwed into a tube mount which can be pushed in or out of a wooden holder for focussing purposes. Colour screens and dia- 
phragms are carried in a recess on the other side of the holder, which is fitted with a dovetail slide for transverse motion on a cross-piece of wood carried on a vertically extensible brass tube-an ordinary telescopic bull's-eye carrier. These two motions provide a rough means for centring the condenser.

An important point in both these arrangements is the means adopted for removing the camera when it is desired to examine the object directly through the microscope. In the first, its carrier slides off the baseboard; in the second, the camera can be lifted off bodily, but in either case it can be replaced without any disturbance to other parts of the apparatus. In other cameras the microscope and.illuminant are carried on a turn-table which can be readily swung in or out of the optical axis; but this plan is rather difficult for an amateur to carry out satisfactorily unless well provided with tools. In professionally made outfits such as that of Mr. Barnard (Fig. 8), the turn-table works excellently.

Focussing Arrangements.-A long extension is of great advantage, in that it permits of a wider range of magnification from one combination of objective and eyepiece, and when a short camera has to be utilised it may even be advisable to add an extra bellows extension, such as is made by Messrs. Lancaster and other firms, to the back, or a conical tube blackened inside, to the front. A long bellows necessitates the use of a mechanical focussing gear to reach the fine adjustment of the microscope while examining the image on the ground glass. In the camera above described it consists of a brass rod carried on two bearings screwed to the baseboard; a milled head is soldered on one end and a pulley on the other. A piece of thread is used to form a band round the pulley and fine adjustment head, and motion is thereby conveyed with very fair delicacy to the fine 
adjustment, but no mechanical focussing device gives quite such delicate control over the fine adjustment as is obtained by direct manipulation of the milled head. Other focussing arrangements are shown at Figs. 41 and 46 , and a well-known device for the purpose is a Hooke's handle (Fig. 67). A little play must be allowed in the gear wheels connecting an unsupported handle to the fine adjustment, otherwise the focus will be upset when the rod is laid down.

Screen for Light Adjustment.-An easel covered with white paper forms part of the apparatus shown in Fig. 2. The camera can be removed and replaced by this screen for the preliminary adjustment of the light, as it is easier to detect uneven illumination on the white surface than on the ground glass. The image is also more readily examined and focussed on the white paper, and it is possible to place the focal plane of the camera exactly in the position originally occupied by the screen by means of a scale fitted along the baseboard, and pointers on the camera back and easel. The scale is also of use for determining by calculation from the camera extension the magnification produced by any objective and eyepiece whose powers are known.

Light-tight Connection between Microscope and Camera.The connection between the camera and microscope must be light-tight. A velvet sleeve with an indiarubber ring sewn into one end to fit over the eyepiece, and the other end joined to the camera, answers the purpose very well; or a metal or cardboard ring about an inch deep can be fitted into the lens aperture of the camera, and a cell into which it slips placed over the eyepiece. One of these cells is shown in Fig. 1.

Focussing Screens.-The ground glass fitted to the camera back for focussing purposes should be as fine as possible, in order that delicate structures may be made 
out. Tyler's micro-grain screens are very suitable, and they usually have a clear glass centre. If there is no clear space on the ground glass, a cover slip should be cemented with Canada balsam on to its centre, as it is so much easier to focus fine detail accurately on clear glass than on ground glass, however fine. A focussing lens is used to see the image under these conditions, and it is necessary to make sure that it is focussed on the glass and not on an aerial image in another plane. For this purpose a scratch is made on the clear glass, or lines drawn across the ground glass before the cover glass is cemented on, and these are kept in focus at the same time as the image. Some workers prefer a clear glass screen which can be substituted for the ground glass for final focussing, after the preparation has been searched and properly centred on the latter. Lines are ruled diagonally across the ground glass to aid in centring the light or the image, and it is also advisable to rule rectangles of lantern-slide size, and of the sizes of all the plates that may be used, to coincide with the positions of the adapters in the dark slides.

Centring the Apparatus. - The greatest care must be taken when making the apparatus to ensure that the optical axes of all the parts are truly in line; if this is done at the outset all the components can be placed very rapidly in their proper positions, and only very small adjustments of the lamp or of the condensers, by means of their centring screws, are necessary before taking a photomicrograph. The most careful measurements are hardly sufficiently accurate for this purpose, and the directions given on page 145 for centring the microscope, lamp, and condensing lenses should be followed out before their positions are definitely fixed.

Watson's Laboratory Camera.-The two cameras just described were made without any expert assistance, 


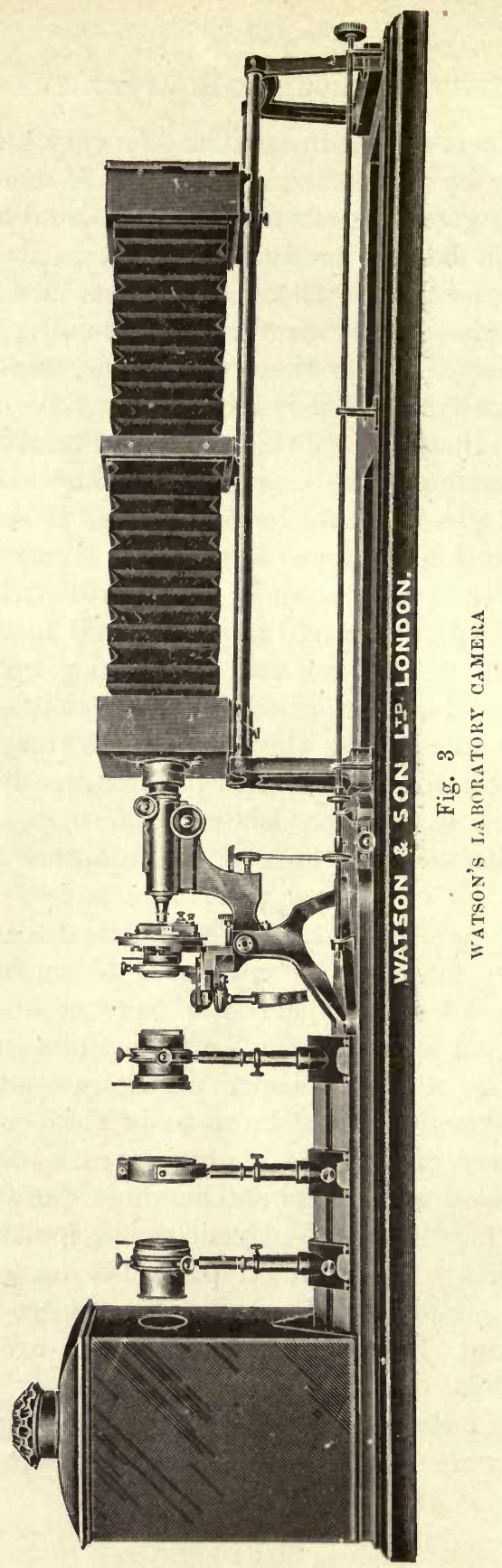


and lack some of the delicate movements provided for centring condensers, etc., in some of the better professionally made outfits, and it is particularly in refinements of this sort and in general perfection of detail that the latter prove their superiority for difficult photomicrography. Fig. 3 shows a camera built by Messrs. Watson on the same lines as the other two, but with improvements in detail. The optical bench is of triargular section, and on it the condenser, diaphragms, and $\approx$ Kingsford trough are carried by means of saddle stands with vertically extensible pillars and centring screws for the condensers. The condenser is the Watson-Conrady system, as described later. The camera can be moved along its supporting rails-made of steel tubing-either from the back or front. Almost any sort of illuminant may be mounted on a suitable stand and used inside the case, and it may be well to point out that some such casing or lantern ought always to be used to enclose the illuminant, as it is much easier to focus when all extraneous light is shielded. The support next to the lamp carries the condenser and iris, the next is the trough, and the third an iris diaphragm, so mounted that a supplementary condensing lens can be fitted into it when required.

\section{Vertical Cameras.-Adaptation of Hand and Stand} Cameras.-The stand, with blocks running in a groove, may very readily be used in a vertical position, as shown in Fig. 4, provided that the back of the camera can be fixed rigidly at right angles to the baseboard. The camera shown is the "Convertible Britisher," arranged for reflex instantaneous photography. A slotted angle-iron is screwed on to either side of the carrying block, and two long screws are attached to the baseboard; these pass through the slots in such a way as to allow the camera a considerable range of vertical movement. Two thumbscrews suffice to clamp the block 
rigidly at the desired height, their position limits the range of motion. The baseboard is screwed to a wall at such a height that the focussing screen is easily ac-

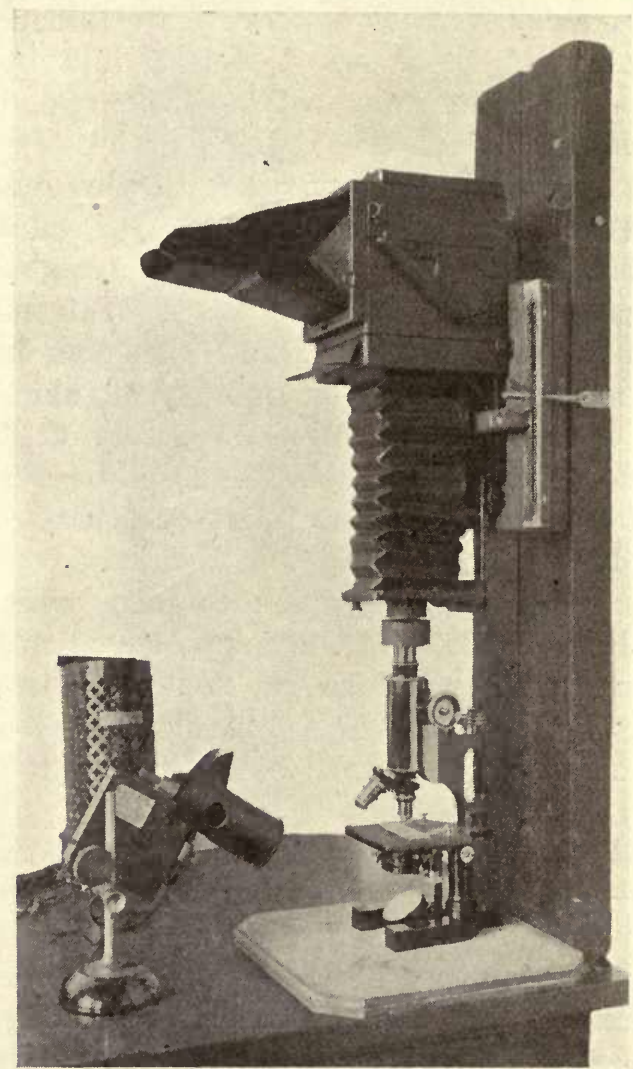

Fig. 4

REFLEX VERTICAL CAMERA

cessible, the microscope and illuminating system being placed on a low table or box. The position of the focussing screen in reflex cameras is a great point in their favour, 
as it permits of the fine-adjustment head being reached even when long extensions are employed.

Van Heurck Camera.-Dr. Van Heurck was the greatest advocate of the vertical system and employed it very largely, and a camera named after him made by Messrs. Watson is a most rigid and efficient instrument. It consists of a box standing on four legs over the microscope, and connected thereto by a velvet sleeve ; it is inextensible, and hence no change in the magnification can be made otherwise than by a change of lenses. There is a door in the front of the camera large enough to allow of the head being inserted to examine the object through the microscope, if desired, or to focus on a white card in place of the ground glass (Fig. 71).

Adjustable Vertical Cameras.-Fig. 5 shows a modification that can be made to fit any suitable camera that may be at hand. It consists of a four-legged support strongly and rigidly made. The top pieces are rebated, so that the camera fits exactly and firmly into them, but is removable at will. The baseboard of the camera also falls firmly into place on the stand, and supports the front rigidly and permits of any desired extension being made with ease. In this it offers an advantage over the Van Heurck model, and, to make use of different extensions easily, two bars are screwed across the legs about $6^{\prime \prime}$ from the ground, and across these a removable board is clamped to carry the microscope, and, if desired, the illuminating apparatus. Another pair of bars can be fitted higher up, or blocks may be provided on which to raise the microscope still more when a shorter extension is used. This stand has proved very useful even for large pictures with an enlarging camera, but generally when long extensions are required the horizontal arrangement is better. In any case, when making use of a particular camera its adaptability to either method should be carefully studied, and 
the stand made in accordance with that to which it is best suited.

Many vertical cameras are on the market which permit

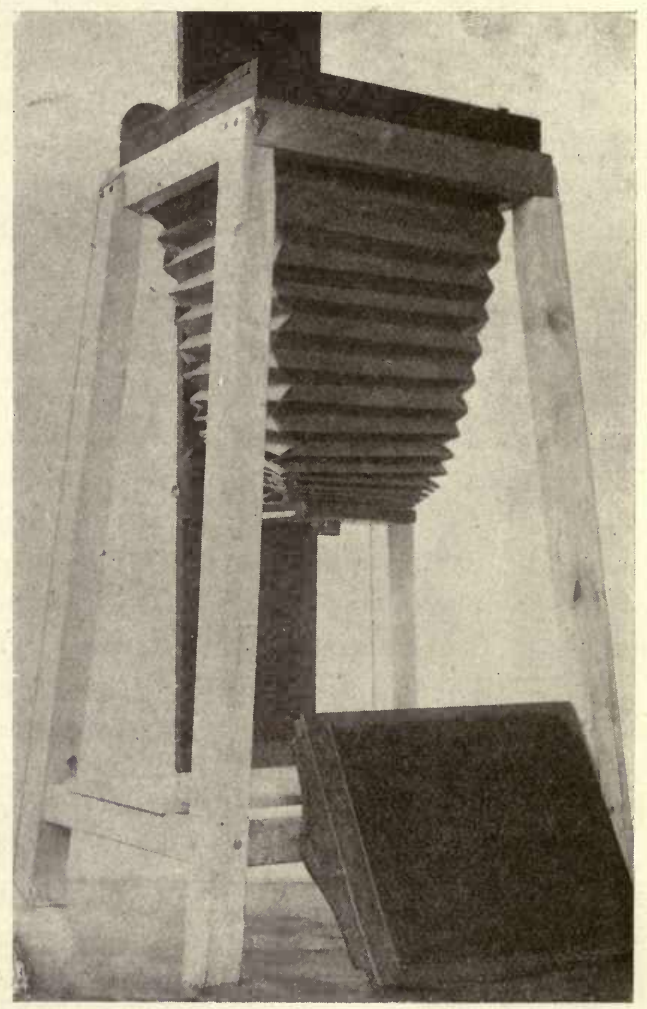

Fig. 5

VERTICAL CAMERA

of a varying bellows extension; some of them are supported by two arms running on a single upright metal pillar, and may be clamped at any desired position on the pillar. These cameras are very convenient to use, but have the drawback that unless very substantially 
<smiles>C1C[Te]C1</smiles> 


\section{Photographs at Natural Size or small magnification with ordinary Photographic and Telephoto Lenses.}

A Embryo Cuck ix SHeLt after 19 days incubation $\times 1$

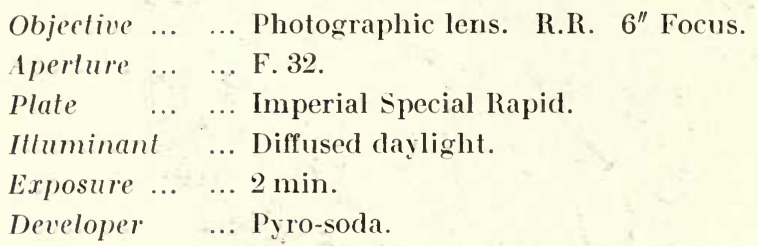

The chick was exposed by carefully cutting away the upper part of the shell under water.

The photograph was taken with the object completcly immersed in water; the shell supported on a base of plasticine.

The black background is a sheet of black paper soaked in methylated spirit previous to immersion in water, to avoid air bubbles rising from the paper.

\section{FossiLs $\times 5 \frac{1}{2}$}

B Hoplites laulus from the Gault, Cambridge, England.

C Potamides cristatus from the Lutetien, Chambors, France.

Objective ... ... Dallmeyer's "Adon" Telephoto, with extension tube.

Aperture ... ... Iris in extension tube closed down to its limit. Extension . ... Image distance, $19 \frac{1}{2}{ }^{\prime \prime}$. Separation of lenses, $5 \frac{1}{2}$ ". Plate ... ... Schleussner's "Paragon." Fine grain ordinary. Illuminant $\quad \ldots 4$ amp. arc, diffused.

Exposure ... ... B. 2 min. Dark-coloured fossil. C. $1 \mathrm{~min}$. White fossil.

Developer ... Netol-quinol.

The fossits were laid on dead black paper and a vertical camera used.

The greaf restriction of aperture is necessary to get covering power and depth of focus, as the "Adon" was made for distant objects. 

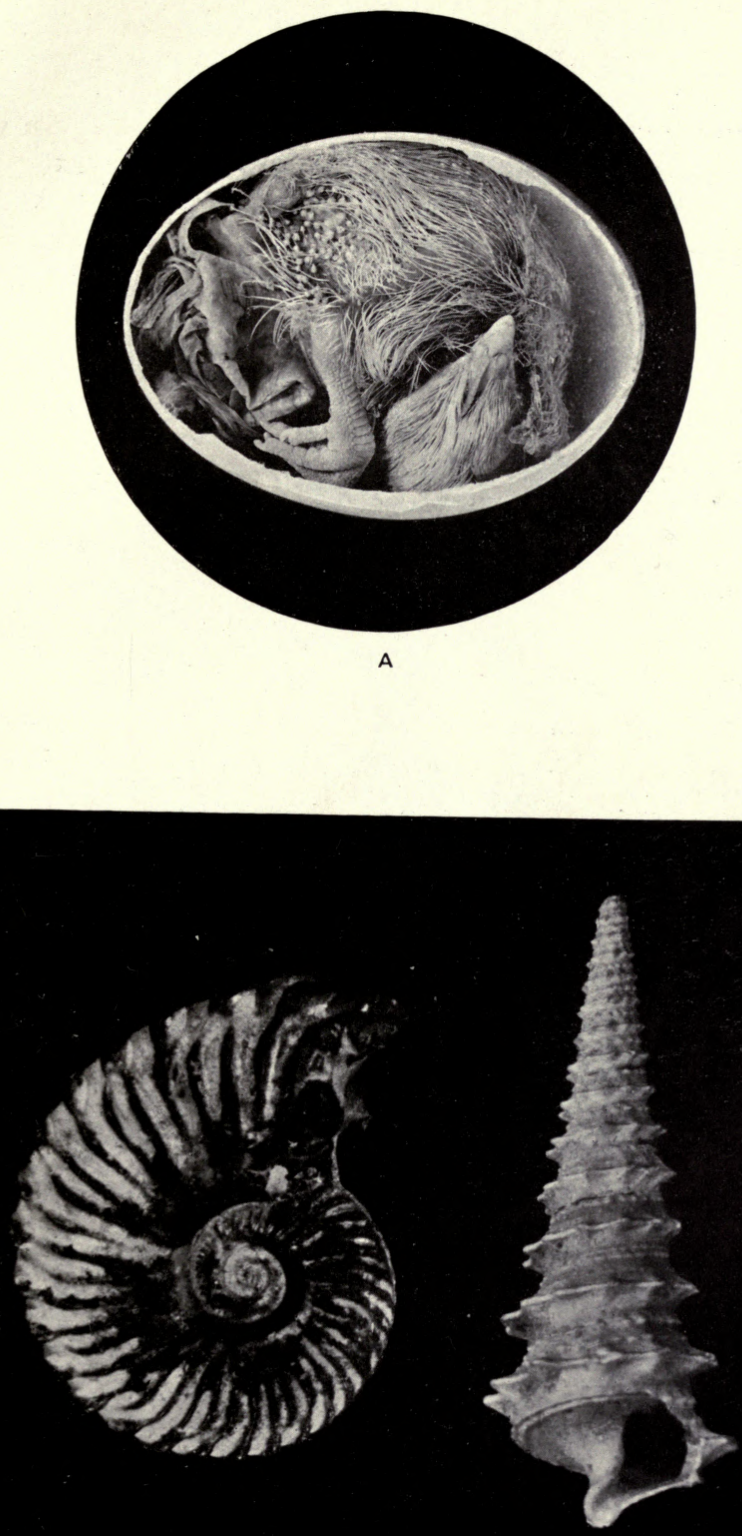

made they are very liable to tremors transmitted from any vibration of the table. The arms that carry the camera must be very rigidly connected to the pillar,

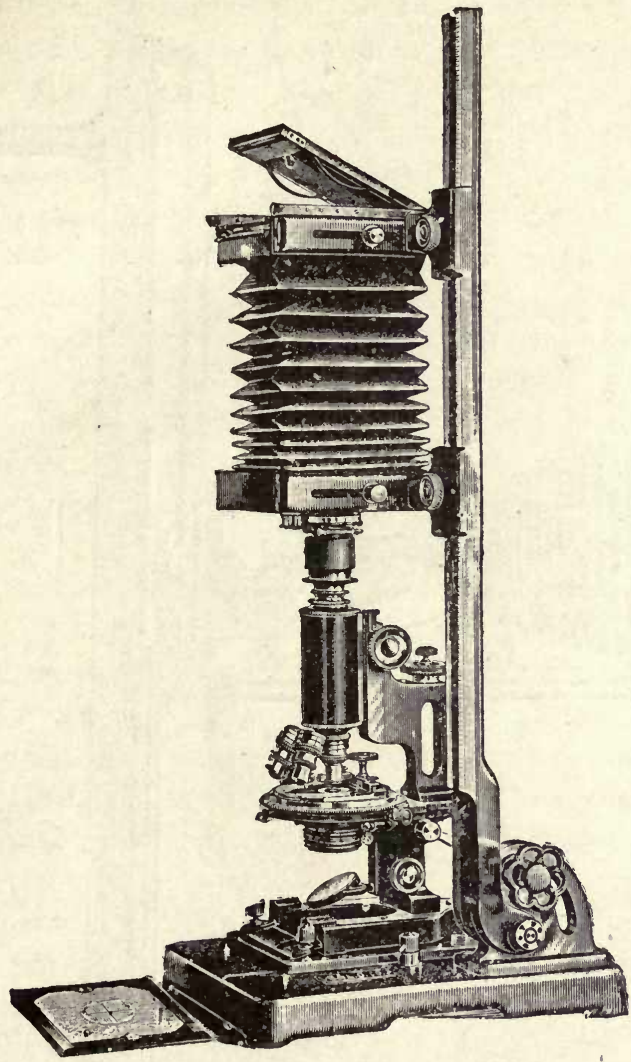

Fig. 6

BAUSCH AND LOMB'S VERTICAL ANI HORIZONTAL CAMERA

or after wear the back of the camera may fall away from its perfectly horizontal position. Cameras carried between two vertical columns are better in this respect.

Universal Cameras. - It is a natural transition from the last two arrangements to an apparatus that may be 
used in either a vertical or horizontal position. This is undoubtedly the most universally applicable form of

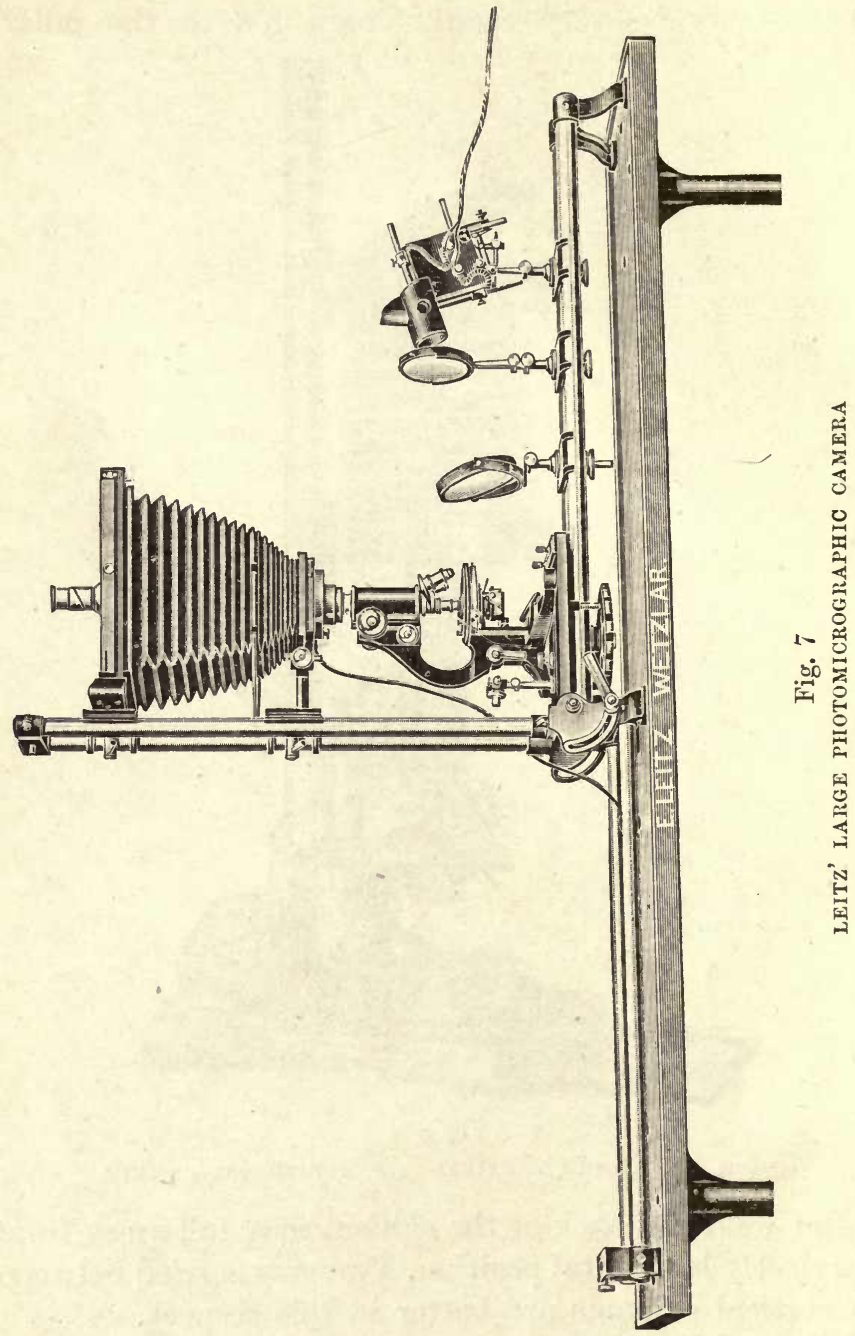

camera, and becomes an instrument of the bighest precision when fitted with an optical bench on which the 
microscope, condensers, and lamp are carried. Fig. 6 illustrates a camera made by Messrs. Bausch and Lomb, and Fig. 44 one by Zeiss. Suitable optical benches can be obtained separately, or are supplied with the camera as a complete photomicrographic outfit such as is illustrated in Figs. 7 and 8. Fig. 7 shows Leitz' large photomicrographic apparatus with the camera raised into the vertical position. A wheel may be noticed beneath the steel tubes which form the optical bench; it is used for vertical adjustment of the stand carrying the microscope when the camera is used in a horizontal position. A flexible rod is supplied for focussing purposes, carrying a little wheel with india-rubber round its circumference, and bearing on the side milled heads of the microscope. The bellows of the camera can be drawn away from the back, if it is preferred to focus on white card.

Mr. Barnard's Geometric Slide Photomicrographic Apparatus.-Mr. J. E. Barnard has designed a very fine photomicrographic apparatus, in which he has employed the system of the geometric slide in the carriers supporting the camera, microscope, and illuminating system. As made by Baker it is shown at Fig. 8. The camera has an extension of 3 feet, and can be moved vertically on rods fixed to the slides; a small white screen is supported on one side, and on it the projected image or disc of light may be examined. A carrier for colour screens is attached to the auxiliary condenser-a Nelson aplanatic bull's-eye. The lamp shown is a Nernst of one ampère. The condenser and lamp are both carried on adjustable centring supports. The rails on which the camera runs are hinged to the main casting, and can be raised into a vertical position; and a strut is provided to hold the camera rigidly over the microscope. The part of the stand carrying the microscope, lamp, and condenser is pivoted so that it can be swung round to enable direct observations 


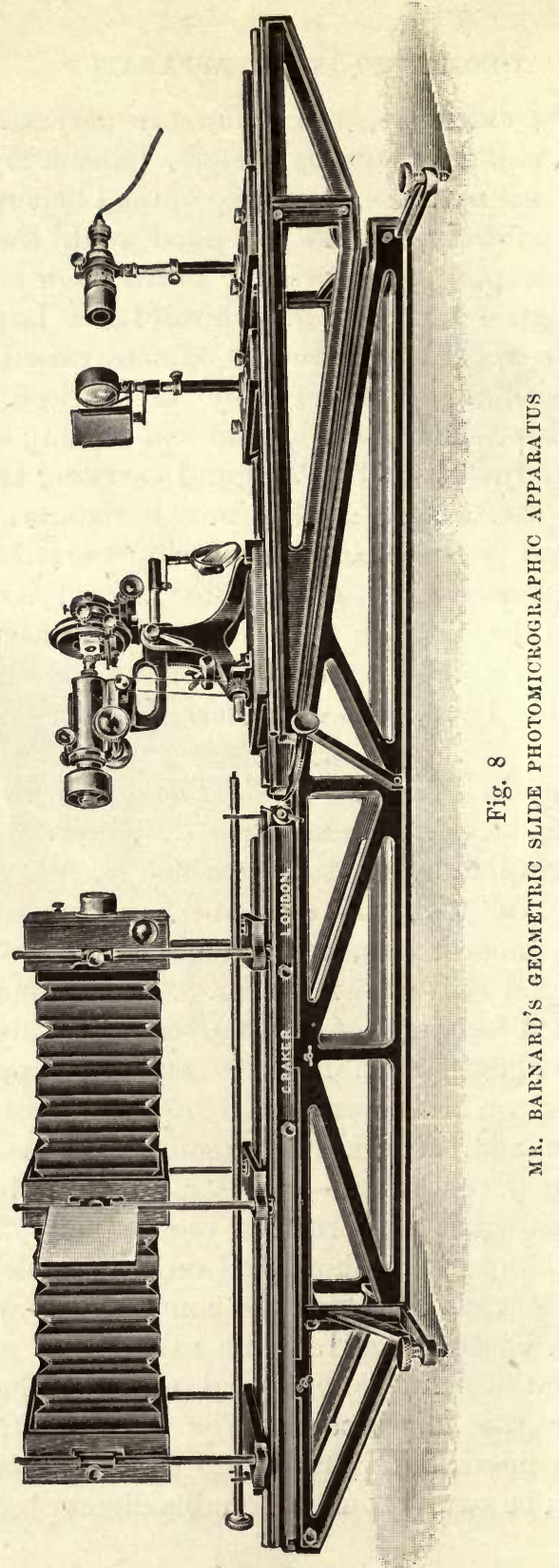


to be made with the microscope, or for projection of the image on to the white screen.

Zeiss' Large Camera. - The large apparatus made by Zeiss is one of the most elaborate and complete outfits made. The camera and optical bench are carried on separate tables as indicated in Fig. 48. The chief advantage claimed for this arrangement is that any vibration given to the camera when moving the focussing

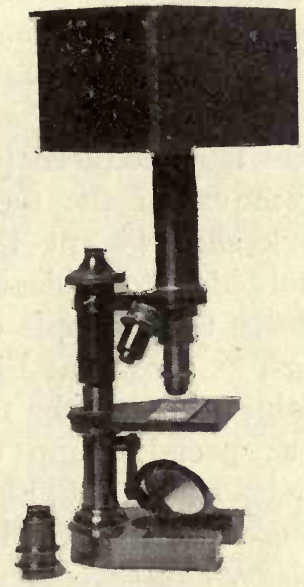

Fig. 9

SIMPLE BOX CAMERA

screen or dark slide is not transmitted to the microscope, and does not upset the adjustment.

Small Cameras.-There are many small cameras made to slip on to the top of the microscope, and intended for use without any further support. Some are box-shaped and others conical, while the Gordon apparatus made by Messrs. Beck is a brass tube with a chamber at its upper end, in which a small plate is placed. The simplest apparatus that we have ever used was made from a small wooden box, the lid of which was removed and flanges fixed so as to receive a focussing screen 
and metal dark slide (Fig. 9). In the opposite end of the box a hole was drilled, and a metal tube affixed of such a diameter as to slip over the eyepiece end of a microscope; this was fastened to the microscope tube by a clamping screw. The box was blackened inside and rendered light-tight, and answered quite satisfactorily for low-power work. Although we do not advocate the use of quite such simple apparatus as this, yet in place of better, in the hands of a careful worker, it is capable of giving good results. To illustrate this a photomicrograph of a diatom (Pleurosigma angulatum) taken with this particular apparatus, using a $\frac{1}{12}$ inch objective, and No. 41.c eyepiece is reproduced (Plate 41). Unless care is used, the removal of the focussing screen and insertion of the dark slide is apt to alter the focus, especially if moderately high magnifications be attempted, and it is better to have these simple cameras supported on three or four legs, as shown at Fig. 10, particularly as their weight is liable to cause the microscope tube to slide down, unless a special clamp to prevent this is supplied. The camera shown at Fig. 10, although in essentials nothing more than one of these small cameras supported on four legs, has several points of interest. It is a simplified reflex camera of quarter-plate size. The ground glass is at the side, a very convenient position when the camera stands on a table, and the image is projected on to it by a mirror which turns aside before the exposure is made by means of a flap-shutter controlled by a release.

The Workroom.-A workroom set apart for Photomicrography is not often available, but whenever possible a table, shelf, or cupboard should be reserved for the apparatus, which can then always be kept ready for use. The dark room is invariably somewhat damp, and consequently it is not advisable, even when space permits, to keep the apparatus there. The best arrangement 
is that of a dark room of comfortable size adjoining the workroom, and the latter should be fitted up with curtains, so that it can be darkened when desired. Ready access to every part of the apparatus must be assured, and

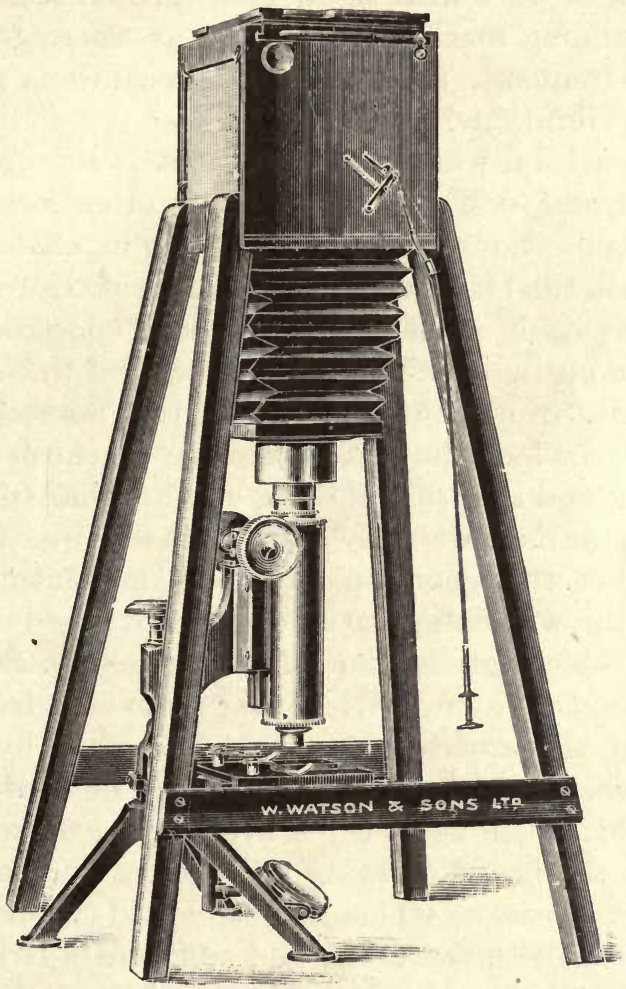

Fig. 10

SMALL VERTICAL CAMERA FOR INSTANTANEOUS EXPOSURES

comfort, without which delicate operations are almost impossible, must not be neglected. Draughts and great fluctuations in temperature should be avoided, as liable to upset fine adjustments.

Prevention of Vibration.-Vibrations are very detri- 
mental, and every care should be taken to select a room as far away from disturbing influences as possible. A basement room is freest from vibrations, and a stone floor less liable to convey them than a wooden one. It is almost impossible to work with any degree of comfort near heavy traffic, running machinery, boilers, or where banging of doors is frequent, unless special precautions are taken to avoid vibrations.

The bench on which the apparatus is set up must be of the firmest description, and it is often less liable to transmit vibrations if carried on strong brackets attached to the wall, than if placed in the middle of the room. The apparatus itself must also be solidly constructed, and supported on india-rubber pads or several thicknesses of carpet felt. Sufficient rigidity in the microscope and camera is in fact the most effective precaution against vibration, and as a rule nothing further than the rubber or felt pads is required. But if these are insufficient to prevent the communication of movement to the camera, the whole apparatus can be arranged on a stout board or table, and suspended from the ceiling. Suspension is often a space-saving device if the room is high enough to permit of the apparatus being lifted clear by suitable pulleys when not in use. Mr. J. T. Pigg devised a modification which is, in many cases, even more convenient. He suspends the board carrying the camera and microscope by means of catgut violoncello strings to two iron arches firmly screwed to the table, and sufficiently large for the apparatus to swing beneath them. A special bench in the basement built on masonry foundations, and insulated entirely from the building, is the best support for photomicrographic apparatus, when circumstances allow of such an elaborate arrangement. 


\section{CHAPTER II}

THE MICROSCOPE

As the question of cost is usually one of the chief considerations governing the choice of a microscope, it can hardly be ignored here, and any advice on the selection must of necessity be qualified by the price which the purchaser is willing to pay. The particular requirements of the branch of microscopy in which the worker is most interested must first be considered, and the mechanical or optical arrangements of a certain stand may point to its selection as the most suitable in preference to others of equal merit, but differing in detail. A microscope so selected will generally do for photomicrography, although it must be borne in mind that the calls to be made upon it are more exacting than those usually demanded by visual work.

Petrological, metallurgical, and other microscopes of special construction are not considered in the present chapter, and attention is confined to stands of the ordinary form intended for general use. The chief points to which attention should be directed are mentioned, but it is not to be expected that all the movements and fittings described will be found in any one stand unless a very high price is given, and a microscope comprising a selection of the most necessary is quite as useful for most work, and its greater simplicity is even an advantage. But it is remarkable how some of the adjustments that will be referred to as luxuries become almost necessities to a worker who has become accustomed to 
their use. The soundest advice is to buy at the outset the best stand that can be afforded, even if its price involves the sacrifice at first of one or more lenses. Convenience and pleasure in use, combined with accuracy in the movements and good wearing qualities, well repay the initial outlay, and further objectives can be added at any time.

The Stand.-The first essentials in any microscope, however simple or elaborate it may be, are freedom from tremor, and rigidity in the vertical or horizontal positions when clamped to the table or baseboard. Many stands, even of the best makes, will fall forward when the tube is placed horizontally, and it can hardly be claimed as essential that they should not, as a clamp easily gets over the difficulty, but at the same time it is a great advantage to find a stand that will not require clamping. Two types of foot are described in connection with the illustrations, "Tripod or Claw" and "Horseshoe," the former generally gives the more rigid support. The horseshoe must be very substantial, with the single toe projecting some distance behind the pillar, to give proper support to a horizontal microscope.

Microscopes are now very often finished in black, instead of brass, and the appearance is very good. The only thing that can be urged against the practice is that the black may cover defects in brasswork, but we have not known this to occur.

The Limb.- The shape of the arm which is attached to the foot and carries the tube and stage is not of great importance, provided that it holds the tube rigidly, but it is very conveniently made in such a way that it can be used as a handle for lifting the microscope without risk of injury to any of the adjustments. Its size should permit of large specimens being placed on the stage, and allow for sufficient up-and-down movement of the tube to focus $3^{\prime \prime}$ objectives as well as high powers. 
The Mechanical Stage.-A mechanical stage is not at all an essential, although of great convenience for searching a slide, and for accurately placing the part to be photographed in the most suitable position in respect to the dimensions of the plate. For the latter purpose a rotating stage is exceedingly useful, and indispensable for petrology. Verniers or scales on the stage movements are most useful for recording the position of any particular spot on a slide that may be required for future reference with high-power objectives. But with all its convenience a mechanical stage is far better dispensed with than other parts of the microscope to be mentioned, and if its addition involves the purchase of a less accurately finished instrument, there should be no hesitation about omitting it altogether; with practice it is quite easy to give a most delicate movement to a slide with the fingers if it is held on a good flat stage by means of clips. If no mechanical stage is fitted, either a square or circular and preferably rotating stage may be selected, according to requirements, and assurance should be made that the stand is suitable for the addition of an adaptable mechanical stage. Most makers provide excellent stages of this description to be added to existing stands, for instance, the stage shown with the Leitz stand at Fig. 16. Another very reliable one with which we are familiar is made by Ross; it may, if desired, be fitted with a micrometer arrangement for measurements. On the whole, mechanical stages built into the stand are preferable to the adaptable type, which are more liable to wear slightly loose or out of true. A very good compromise, combining the rigidity of the built-in and the convenience of the adaptable, is fitted to the Zeiss and Baker stands shown at Figs. 15 and 17. In these the motion to and from the limb is built in, while the transverse movement is given by means of a bar which can be very firmly attached and readily removed when 
a large plane stage is required. To be perfectly finished a rotating stage should be graduated and fitted with centring screws, and a complete mechanical stage would have milled heads for the rotating as well as the rectangular movements. Such refinements are only found on the most expensive stands, and for most purposes are not required. The aperture in the stage ought to be of sufficient size to allow of the use of any condenser that may be required, and there must be no tendency to foul the latter at any point of the traverse of a mechanical stage.

The Substage.-The fittings situated beneath the stage for carrying the condenser and its accessories, should comprise suitable movements for placing the condenser in the best position for illuminating the object, and these are almost as important in a photomicrographic stand as the coarse and fine adjustment of the tube. The simplest form is a fixed sleeve, in which the condenser slides, but this is not adequate for accurately focussing the latter. The better microscopes have a mechanical focussing substage consisting of a ring or sleeve raised and lowered either by a quick-acting lateral screw (Fig. 4), or by a rack and pinion (Fig. 17). The rack-and-pinion arrangement is to be preferred, but the quick-acting lateral screw is a fairly efficient substitute and usually fitted to the cheaper stands. Some of the most complete substages have a fine adjustment as well as the rack and pinion, but this is not often required.

An Iris diaphragm is an essential part of an illuminating system, and is either fitted to the condenser mount itself, or forms an independent part of the substage. An iris is also fitted in the thickness of the stage of some microscopes, or supplied as a cylinder fitting over the condenser (Figs. 15 and 16), and is a most useful accessory.

The substage is sometimes made to swing out of the optical axis when racked down to its limit, and this is 
very convenient for changing condensers, etc. On the other hand, there is the fear that after some wear the condenser will not return to its correct position unless the fitting is very substantially built.

Centration of Condenser and Objective.-Some provision for centring the condenser with the objective is necessary for delicate work, and centring screws are, in many cases, fitted to the substage ring or to the condenser mount itself for this purpose. On many stands, however, no centring screws will be found, and, in the absence of a centring nose-piece, reliance must be placed on the accuracy with which the maker has adjusted the microscope. In such cases the centration of the condenser should always be carefully examined (ps ge 145). Unfortunately it leaves much to be desired on some of the cheaper stands, and, however well it has been carried out, it is only accurate for the condenser and objective originally fitted, and the absolute necessity of centring screws to the substage or nose-piece becomes obvious when either condenser or objective is changed. When a condenser is found to be slightly out of adjustment in a non-centring substage, rotation in the ring may bring it into more accurate alignment with the objective. The absence of centring screws is often associated with an Abbe illuminator, as that does not require such accurate adjustment as an achromatic condenser.

Some workers prefer to attach the centring screws to the nose-piece which carries the objectives, claiming the advantage that once the lamp, condenser, and objective have been accurately centred, any change of objective requires only a slight adjustment of the nose-piece, whereas it may be necessary to adjust the lamp as well as the condenser to get them all in line again if the centring screws are attached to the condenser mount; and objectives are changed more frequently than condensers. 
Others maintain that the substage ring is the correct place for centring screws, as the errors of centration introduced by a change of objective are not so great as those usually found when the condenser is changed, and they consider that it is best to take the objective as fixed and centre the condenser to it. Either method is perfectly satisfactory when properly employed, but

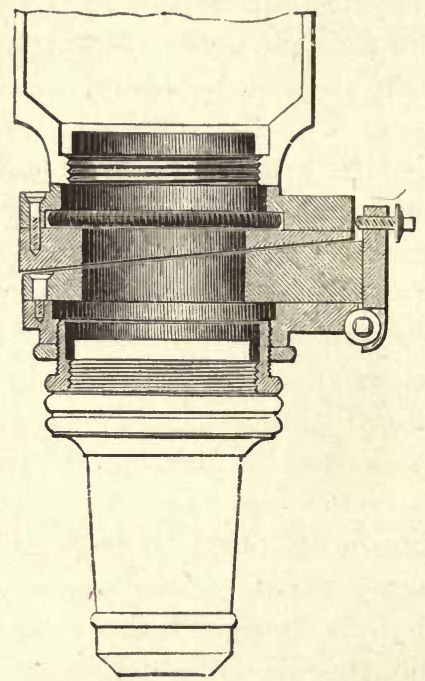

Fig. 11

SLIDING OBJECTIVE CHANGER

there seems less likelihood of improper use when the centring screws are attached to the condenser ring.

Centring Nose-pieces and Objective Changers.-If the microscope has a fixed substage a centring nose-piece should certainly be added. A simple form consists of a ring with centring screws, which screws into the microscope tube, and to which objectives are attached in the ordinary way.

There are several types of nose-piece designed for the rapid change of objectives. The rotating form shown 
in Figs. 16, 17, etc., is that most generally adopted, and is very convenient, but has the drawback in common with one or two other devices for the same purpose that reliance cannot be placed on a proper centration of the objectives when it is used. The sliding objective changers of Zeiss shown on the stand at Fig. 15, and in section at Fig. 11, allow of an almost instantaneous change of objectives, and at the same time permit of their accurate centration by means of screws. A slider is attached to the microscope tube, and each objective is screwed into another provided with centring screws, and so can be rapidly slipped into position.

The Mirror.-The mirror is always provided with both plane and concave reflecting surfaces, and should be carried on a swinging arm capable of universal movements. Some sort of catch to show when the arm is in the plane containing the optical axis is useful, and there should be some means of raising or lowering the mirror to focus the light when using the concave side without a condenser.

The Coarse Adjustment.-The focussing arrangements are the most important mechanical details of a microscope, and the fineness of motion which they impart to the tube must be suitable to the power of the objective in use. On most stands there are a cbarse and a fine adjustment, the former actuated by a diagonal rack and spiral pinion. But some students' microscopes are focussed by means of a sliding sleeve, a poor substitute, however, for a good mechanical arrangement.

The coarse adjustment should be sufficiently delicate to focus low-power objectives, and if well made it should work very smoothly and without any shake. There must also be no tendency for the tube to slide down by its own weight. The range of motion allowed ought to be sufficient to permit of the use of long-focus objectives. An extra range for lenses of 3 " focus or 
over is obtained by screwing the lens into the bottom of the draw-tube, which can be racked further away from the stage than the outer tube.

The Fine Adjustment.-The fine adjustment calls for most eareful attention when choosing a microscope, and can only be dispensed with for very low powers. Most makers have a form of their own, but it is unnecessary to describe them in detail, as the catalogues generally give figures and explanation of the types employed, and a collected description of the principal patterns will be found in Spitta's Microscopy. Most of them depend primarily on a micrometer screw which causes the tube to move up or down on a slide. The slide in the typical Continental pattern (Fig. 9) takes the form of a sleeve fitting over a triangular prismatic bar, and the micrometer screw generally bears directly on the prism, and moves both the tube and limb. This is a very smoothly acting arrangement, but, in some forms, is open to the objection that, if the microscope is lifted by the limb, the micrometer screw may be damaged. Several manufacturers make special provision against this however, by completely casing the prismatic bar and slide, or by attaching a small handle to the solid part at its base. But, in any case, the pitch of a direct-acting screw is limited to about $\frac{1}{10}$ or $\frac{1}{100}$ of an inch, in order that it may withstand wear and tear, and carry the weight of the limb and body. The motion such a screw imparts is too rapid for high-power wide-angle objectives. One rotation of the milled head usually raises or lowers the tube $\frac{1}{50}$ or $\frac{1}{70}$ of an inch, the screw of $\frac{1}{100}$ inch pitch being less frequently used.

In other microscopes the slide is a dovetail fitting carrying the tube and coarse adjustment only, and the limb may be lengthened to make room for a larger stage, or strengthened to form a handle, without adding to the weight that the fine adjustment has to lift. Motion 



\section{Direct Colour Photomicrograph.}

Screen-plate process (Dufay).

Part of the "Eye” in Tall Feather of Peacock $\times 12$

Objective ... ... 3" Achromat.

Ocular ... ... Watson's B.

Illuminant ... Sunlight.

Condenser ... Bull's-eye, projecting convergent beam.

Screen $\quad . . .$. Dufay compensating screen for daylight.

Plate $\quad \ldots \quad \ldots$ Dufay colour plate.

Exposure ... ... 30 secs.

Photomicrographs in colours of opaque objects are more difficult to take satisfactorily than a subject illuminated by transmitted or polarised light. It is necessary to have a very strong illuminant, in this case sunlight. 


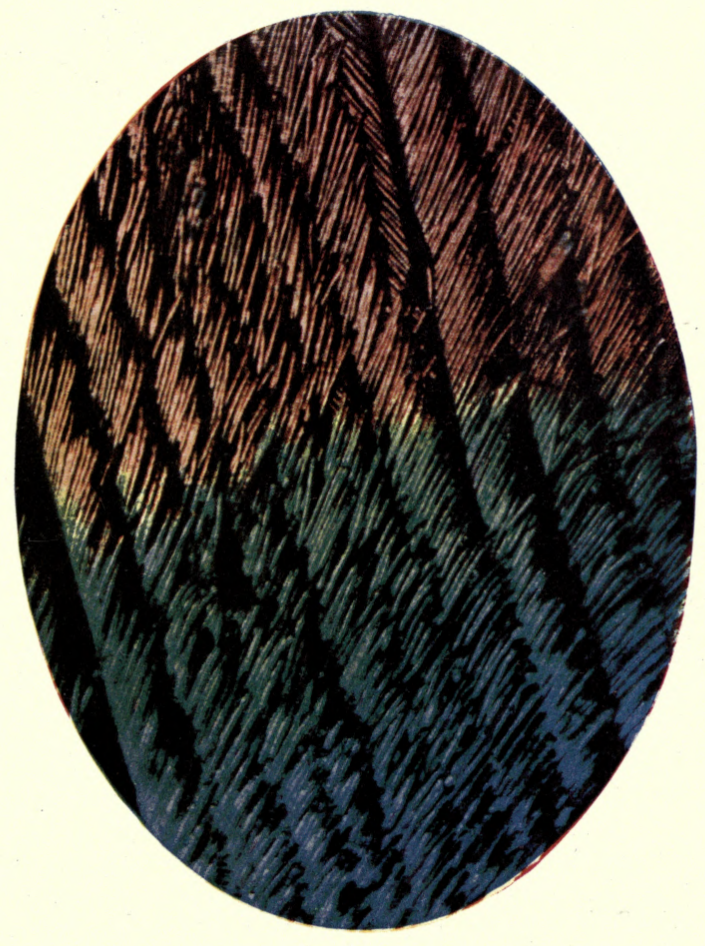






\section{Photography of Opaque Objects.}

A Group of Marine Shells from the W. Donegal Coast $\times 8$ Objective ... ... Crouch $2^{\prime \prime}$ Achromat. Stopped down. Ocular ... ... None.

Illuminant ... Sunlight reflected from a concave mirror and focussed on to the object.

Plate $\quad \ldots \quad \ldots$ Imperial Special Rapid.

Exposure ... ... $20 \mathrm{sec}$.

A small diaphragm was placed between the component lenses of the objective to give depth of focus.

Diameter of aperture of diaphragm, $1.5 \mathrm{~mm}$.

\section{Photography of Plate Cultures of Micro-organisms.}

Dark ground illumination.

B Penicillium glaucum, young colony of mould on gelatine plate $\times 25$

Objective ... ... Seibert No. 0. $36 \mathrm{~mm}$.

Ocular $\quad \ldots \quad \ldots$ Seibert I.

Condenser ... Abbe, with wheel stop.

Illuminant ... Sunlight reflected from the mirror.

Plate $\quad \ldots \quad \ldots$ Ilford process.

Exposure ... ... $5 \mathrm{sec}$.

Petri dish, without cover, laid on microscope stage. 

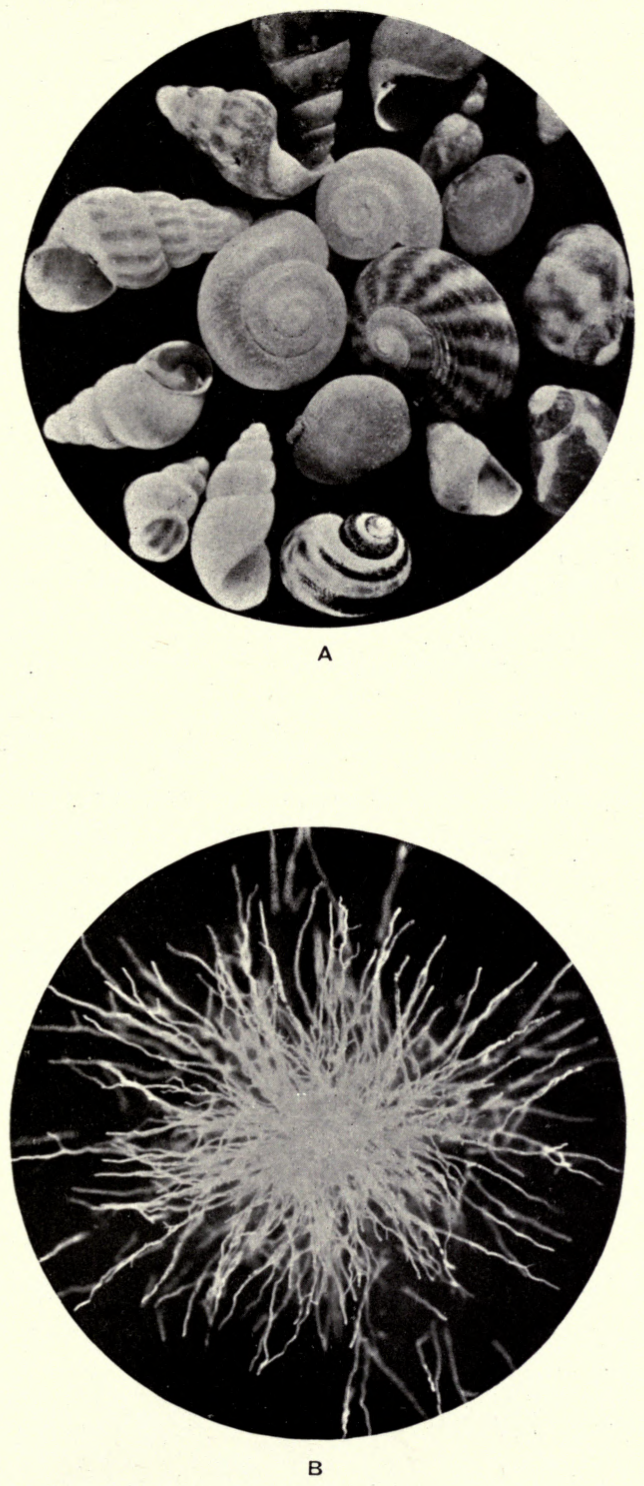

in this case is conveyed from the screw to the slide through an intermediate lever or other device which makes the movement slower. Such speeds as $\frac{1}{200}$ or $\frac{1}{30} 0$ of an inch for each revolution of the milled head are usual. A lever is the simplest and one of the most perfect devices, and the ratio of the lengths of its arms governs the rate of motion. One rotation of a screw of $\frac{1}{50}$ " pitch bearing on the longer arm of a lever whose ratio is $5: 1$ raises the other end $\frac{1}{25}$ of an inch, and so moves the tube through that distance. A lever of this description is placed in the arm of the Van Heurck and Edinburgh microscopes (Figs. 13 and 18). Other makers adopt other devices for reducing the motion imparted by the micrometer screw. Zeiss, for instance, employs Berger's micrometer screw, and Leitz a cam whose rotation raises the tube or allows it to fall. Figures and descriptions of these are given in the respective catalogues.

A fine movement of this description is essential for the highest power work, but for low and medium powers a quicker movement is easier to work with, and $\frac{1}{50}$ " to $\frac{1}{10} \sigma^{\prime \prime}$ is quite adequate. If the milled head is graduated, its rotation may be used for measurements in a vertical direction, provided the pitch of the screw is known, or the movement caused by one complete rotation is given by the maker.

The position of the actuating milled heads is not of very great importance, provided that they can be easily manipulated while the hand or arm rests on the table. The lateral position is very popular now, and when using it the hand may be rested on the tripod foot, but it is somewhat easier to connect a milled head situated on top of the limb to a focussing rod.

Testing the Fine Adjustment-Any of the forms or modifications of fine adjustment adopted by makers of repute can be relied on to give satisfaction, but in testing a microscope it is well to pay particular attention to 
this movement. An object such as a diatom should be examined by the highest power likely to be used, and the fine adjustment should be capable of focussing accurately the different planes. There must be no backlash, and any part that is focussed should remain in focus, even if the instrument receives a slight jar, such as a rap on the table might impart, and no lateral movement of the object should be visible when the fine adjustment is used. It is advantageous to have one of the so-called "Safety" types, by which little or no pressure is applied to the cover-glass if the objective is accidentally brought into contact with the slide, so obviating risk of damage to both preparation and objective. In microscopes so fitted the downward movement of the tube depends on its own weight, together with a spring acting against the fine adjustment, and it is well to see that the spring is sufficiently strong to cause the downward motion when the microscope is horizontal.

Tube Length.-English makers used to fit tubes of a fixed length of $10^{\prime \prime}$ to their microscopes, but these are now practically obsolete, as the more compact Continental tube of 160 or $170 \mathrm{~mm}$. is so much more convenient. The modern practice is to make the tubes telescopic, as it is essential, as will be mentioned later when discussing objectives, to be able to vary the tubelength by means of one or two draw-tubes. These drawtubes should be engraved with a millimetre scale, to indicate the total length in use between objective and eyepiece, and it is very convenient to have a rack-andpinion movement fitted to one of them. They ought to allow of the extension being varied from about 130 or 140 $\mathrm{mm}$. to at least $250 \mathrm{~mm}$., to permit of the use of objectives that have been made for the long tube and for adjustment for cover-glass thickness ; many microscopes, however, only allow of an extension to about $200 \mathrm{~mm}$., and this is generally enough, as objectives are now nearly always 
constructed for tube-lengths of 160 or $170 \mathrm{~mm}$., and only to order for $250 \mathrm{~mm}$. An old-fashioned instrument with a fixed $10^{\prime \prime}$ tube should only be bought under special conditions, and if of very superior make, as were those of Powell and Lealand. The cheaper ones are usually very liable to vibrations, and in any case the fixed tube is a disadvantage for refined work.

Diameter of Tube. - The width of the tube is important, as, when working without eyepieces, it governs the area of the projected disc, and, within the covering power of the objective, the size of the object that may be photographed. Tubes are usually made $37 \mathrm{~mm}$. (1 $\left.1_{4}^{\prime \prime}\right)$ in diameter, but for photomicrography $50 \mathrm{~mm}$. is much to be preferred. There are several makes of intermediate size. To take full advantage of the wide tube, provision must be made for the removal of the draw-tube. The interior of the tubes must be dead black, so as not to give rise to reflections.

Illustrations are given of several stands with which the writers are familiar. They are selected to show as wide a range of ${ }^{\circ}$ constructional details as possible, and for convenience in description and comparison divided into three groups, according to the completeness of their mechanical equipment. They can all be very highly recommended, but that must not be taken to imply that others not described are not equally good. The instruments turned out by the well-known makers are of such consistently high standard that the choice of a particular microscope is generally more a matter of convenience, or on account of the presence of some detail not found in others, than of general excellence or efficiency. The makers usually have stands corresponding to each group, and at approximately the prices mentioned below for the stand alone, without optical equipment or accessories of any kind.

Group 1. Large microscopes costing $£ 15$ and upward. 


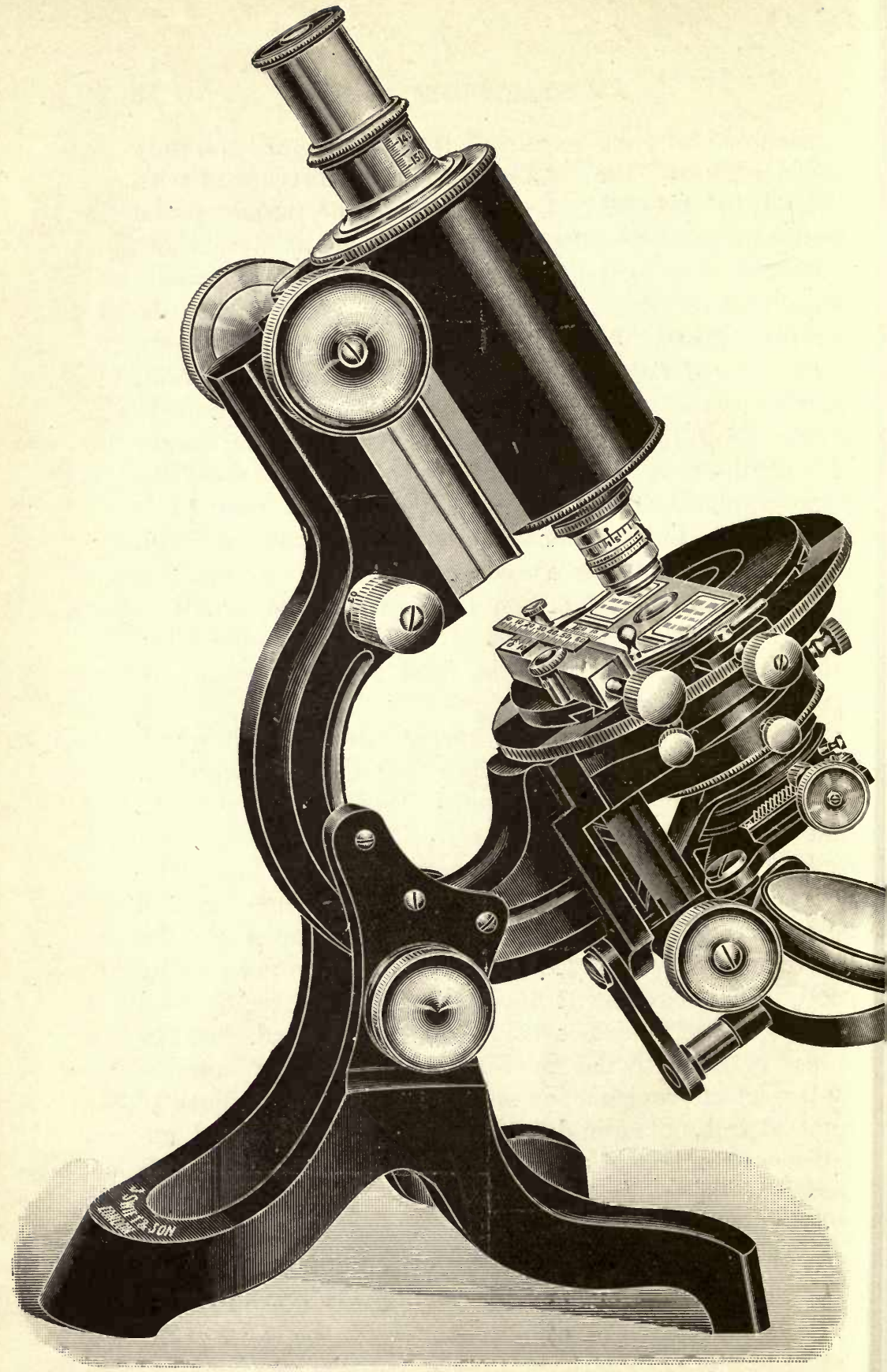

Fig. 12

THE PREMIER MICROSCOPE 
Group 2. Medium microscopes costing about £10.

Group 3. Students' stands at about $£ 5$ or somewhat less.

Large Microscopes.-Six examples of the first-class stands of different makers are given. The first three are more elaborate than the others, and are fitted up with all, or nearly all, the features that have been described as desirable for photomicrography. They are consequently more expensive than the microscopes exemplified by the next three figures, and, with other similarly complete instruments, cost between $£ 20$ and $£ 30$. The next three, Figs. 15, 16, and 17, deserve equally well the name of first-class microscopes. They do not suffer in comparison with those at higher prices on account of any inferiority of workmanship and finish, but they are not so complete in their equipment, although fitted with every essential for critical work.

Swift's "Premier" (Fig. 12).--In the design of this microscope Messrs. Swift had the advantage of a specification given by Mr. Barnard, and the result is an instrument that may be given here as a model of what a photomicrographic stand should be. The "Wales" principle of inclination, in which the limb slides round on the foot, is adopted, and gives a stand that is equally rigid in both horizontal and vertical positions. The foot is a very substantial claw tripod. The tube is $2^{\prime \prime}$ in diameter, and fitted with draw-tubes extending to $250 \mathrm{~mm}$. The mechanical stage permits of complete rotation, is fitted with centring adjustment, and allows of the systematic examination of the entire surface of a $3^{\prime \prime} \times 1 \frac{1}{2}^{\prime \prime}$ slide. The fine adjustment is of a very slow lever pattern actuated by twin milled heads which are graduated.

The substage is focussed by rack and pinion, and centring screws are fitted to the ring into which the condenser slides. There is an Iris diaphragm beneath it with rack-and-pinion motion for oblique light. 


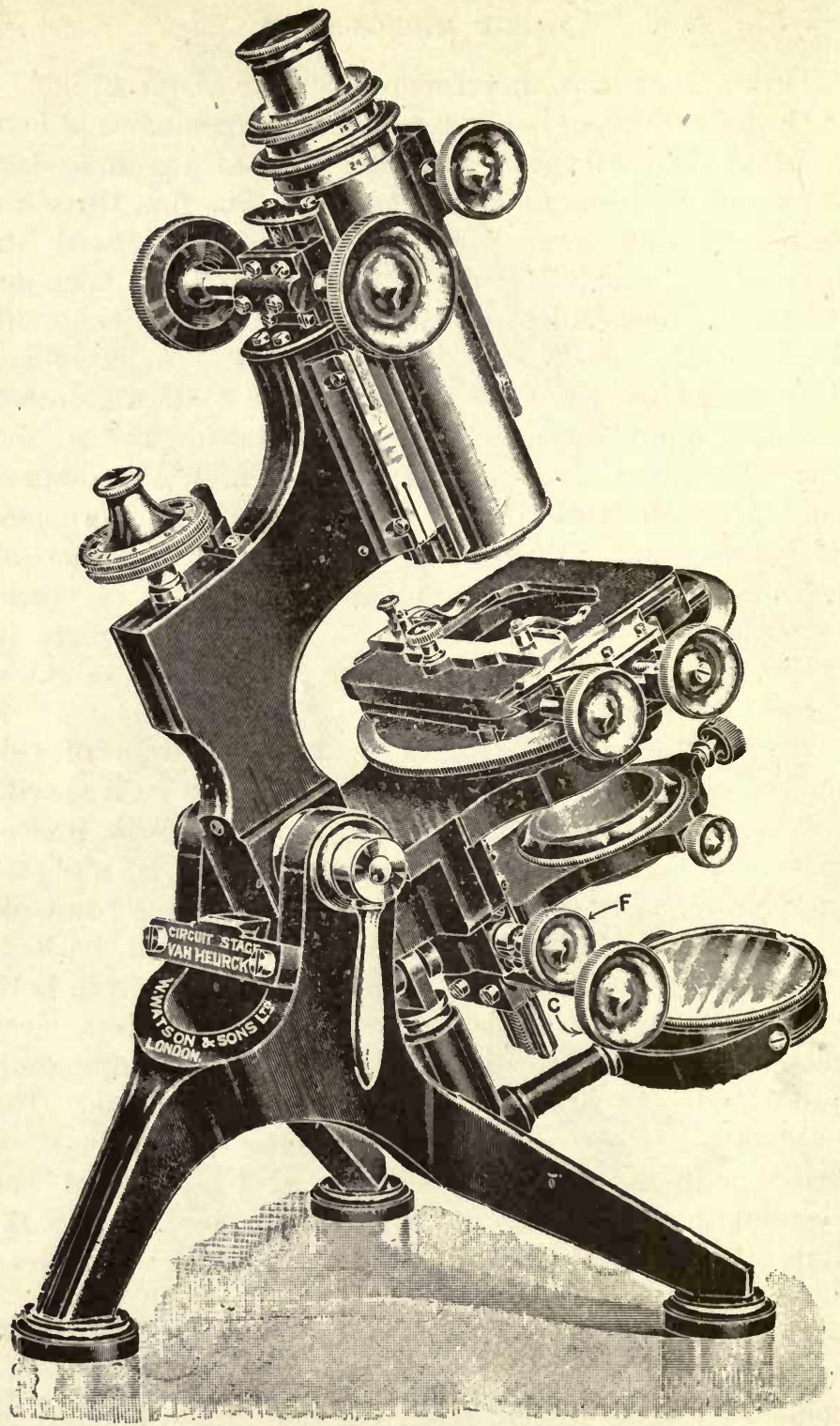

Fig. 13

THE CILCUIT STAGE VAN HEURCK 
The Circuit Stage Van Heurck Microscope (Fig. 13).This is one of a series of three models made by Watson based on designs by the late Dr. Henri Van Heurck, and intended to comprise every movement that can be desired for photomicrography. Being so completely equipped, the stands are very large; the one figured is the medium size.

The suspension of the body on a large tripod foot is typical of English stands, and gives a perfectly steady support both horizontally and vertically. The fine adjustment is Watson's' well-known lever pattern, giving a movement of $\frac{1}{33} 0^{\prime \prime}$ for each revolution of the milled heads. The coarse adjustment allows of the use of 4 " objectives. The stage is very completely equipped with rotating and mechanical movements, and can be fitted with centring screws, verniers, or graduations on the circumference. The substage has centring screws, and is focussed by rack and pinion. In addition a fine adjustment $(\mathrm{F})$ can be fitted if desired. The substage can be turned out of the optical axis for change of condensers, etc.

The Spencer Microscope, No. 10 (Fig. 14), is an admirable example of American practice. A similar stard by Bausch ard Lomb is shown at Fig. 6. The chief difference between the two is in the position of the milled heads actuating the fine adjustment, which are both lever types, but of different construction.

The mechanical stage can be removed from the revolving stage; the milled heads are on concentric axes one above the other. The revolving stage is covered with vulcanite, and fitted with centring screws and a clamp. Its edge is graduated in degrees. The tube is $50 \mathrm{~mm}$. in diameter. The substage is similar in principle to that of the "Premier," but the Spencer Lens Co. fit a second iris diaphragm between the lenses of their condensers. The foot is of the horseshoe form, and 


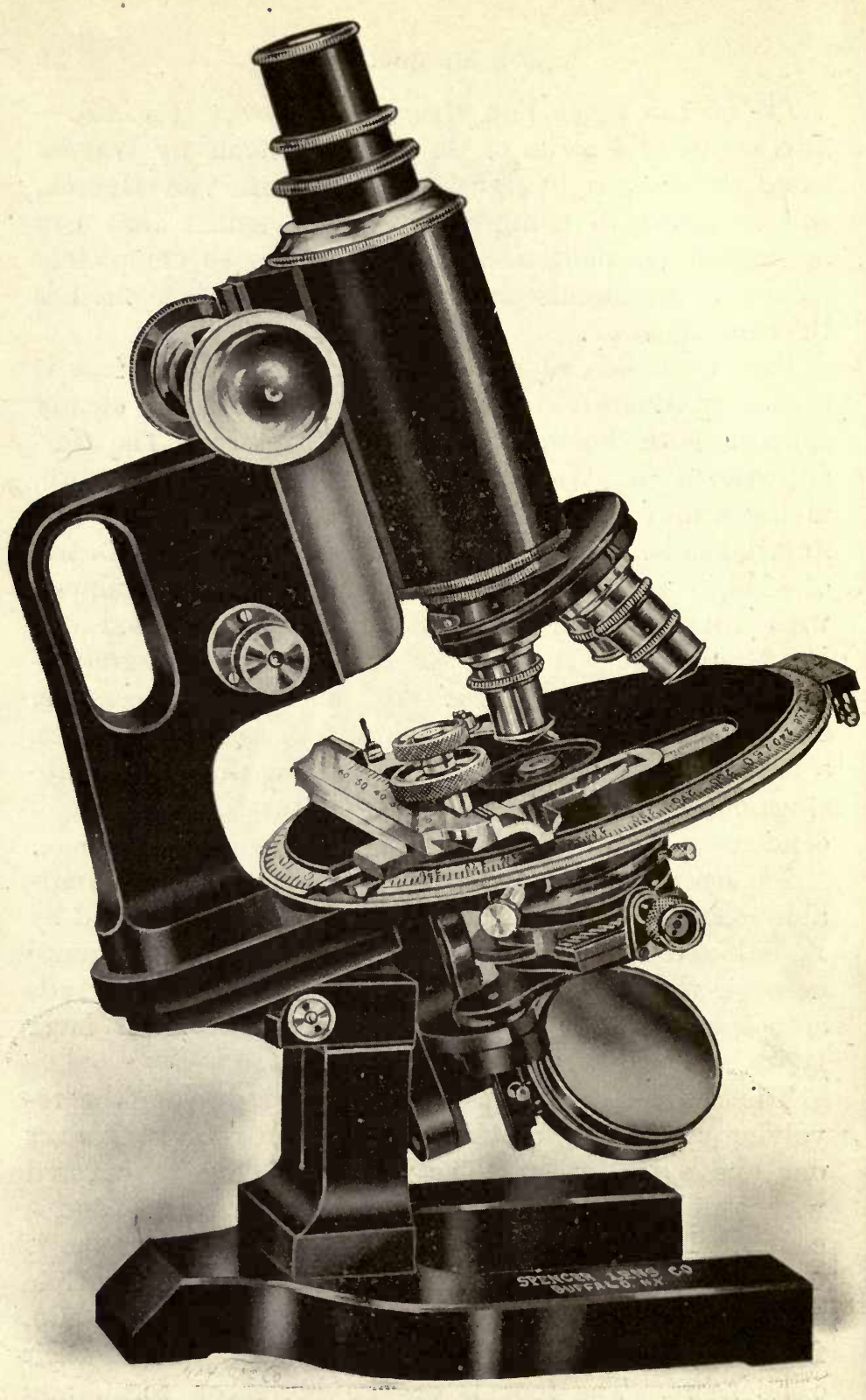

Fig. 14

SPENCER MICROSCOPE NO. 10 . 
although exceptionally massive, it does not give perfect stability when the tube is horizontal unless clamped.

Zeiss, No. 1 (Fig. 15), is a typical Continental stand of the very best construction. The makers supply a special rotating and centring mechanical stage, with very slow movements governed by coaxial milled heads for photomicrography, but the ordinary stage is preferable for most purposes; in this the vertical movement is built into the stage, the bar carrying the lateral movement is removable. The substage is simpler than that illustrated in Fig. 14; it is focussed by rack and pinion, but does not carry centring screws. The Berger fine adjustment is very good, and fitted with lateral milled heads; the body tube is of wide diameter. A simpler stand of similar construction, but without mechanical stage and wide tube, is shown at Fig. 27.

Leitz Stand " $A$ " (Fig. 16) is another fine Continental design with considerable difference in detail from Zeiss' No. 1. The body-tube and fine-adjustment position are similar to the latter, as also is the compact substage arrangement. But the handle-arm is of different form, and gives this stand, and some of the cheaper ones of this make, a distinctive appearance. The mechanical stage is an adaptable one, but of very sound construction; its omission would reduce the cost of the stand by $£ 4$. The stage is circular, revolving, and centring. The fine adjustment is of a special safety pattern depending on the rotation of a cam.

Baker's "R.M.S." (Fig. 17).-This stand is of the English pattern, but simpler than the "Van Heurck." The substage is of the usual rack-and-pinion and centring type, but cannot be swung out of the optical axis; it can be fitted with fine adjustment or not, as desired. In the form of the body, position of fine-adjustment heads, and mechanical stage it resembles the Zeiss No. 1, though differing in constructional details. The fine 

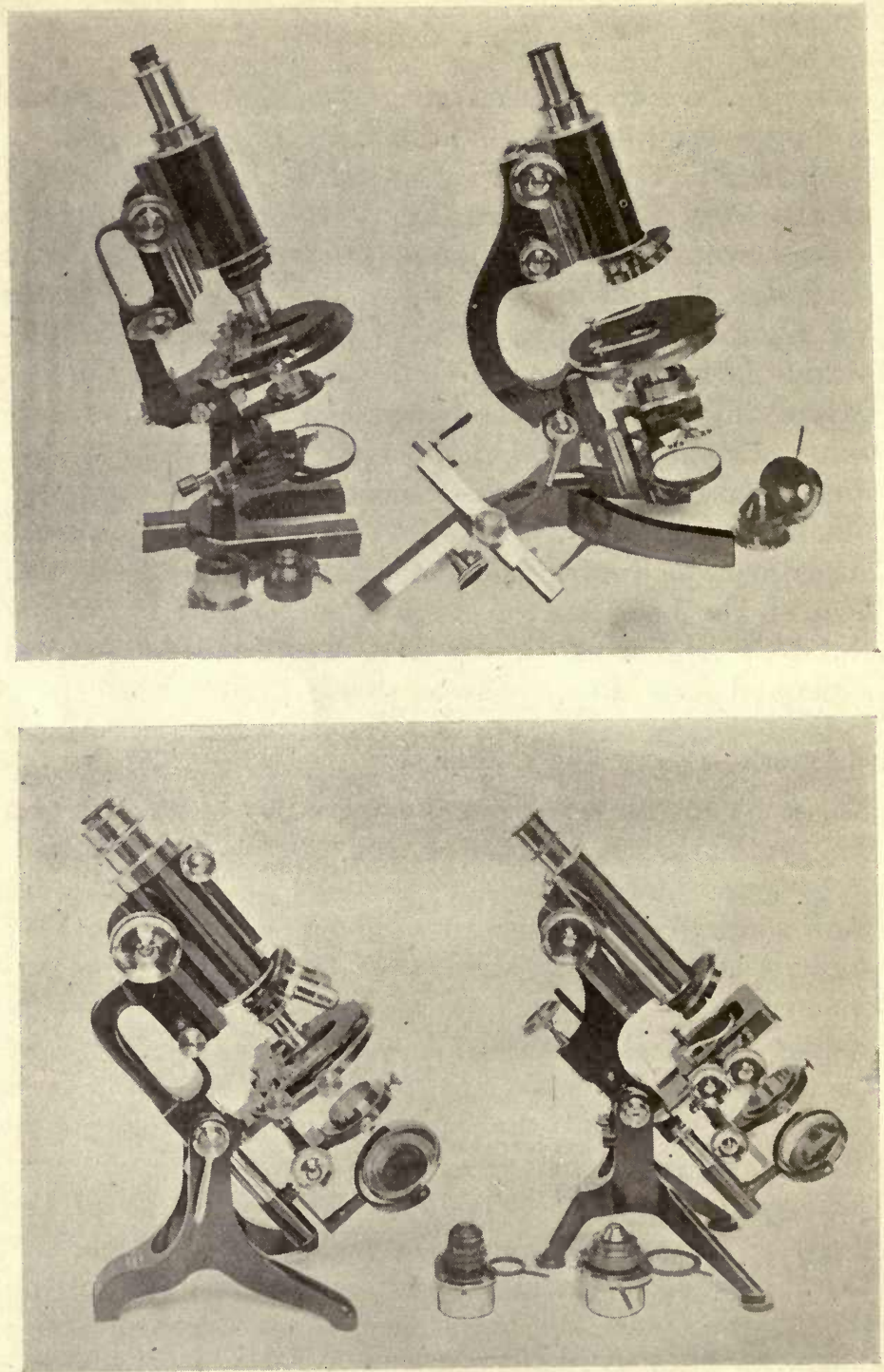

Fig. 15

ZEISS MICROSCOPE No. 1

Fig. 17

“R. M.S.' MICIOSCOPE
Fig. 16

IEITZ MICROSCOPE “A”

Fig. 18

EDINBURGH STUDENTS' MICROSCOPE " H" 
adjustment is of a lever pattern. There is a rack and pinion for adjusting the tube-length.

Microscopes of Medium Size.-The most popular microscopes are undoubtedly to be found among the many excellent stands costing between $£ 10$ and $£ 12$, designed generally on the same lines as the first-class instruments of the same maker, but rather smaller and simplified in mechanical details, in order to bring their price down to a moderate figure. Taken as a group no doubt need be felt as to their capacity to do good work with the highest powers and most refined lenses, but the wide tube and a few of the movements that make the first group so advantageous for photomicrography are omitted.

Watson's Edinburgh Students' Microscope " $H$ " (Fig. 18 ) is given as an example, and shows pretty well what fittings may be expected on a stand of this group. It is built somewhat on the lines of the Van Heurck, and has a built-in mechanical stage, rack-and-pinion swingout and centring substage, and moderately wide tube.

Students' Microscopes.-A photograph of Bausch and Lomb's "B.H." stand is given at Fig. 4 as an example of the third group of microscopes. It has a plain stage and screw-focussing substage, but with no arrangement for centring the condenser. Stands of this class are scarcely adequate for the most serious high-power photomicrography, but for medium and low-power work they are satisfactory. The lack of centring mechanism for the condenser is the most serious drawback. If such a simple stand is bought, it is important to make sure that a mechanical stage can be added later, and that the stand is sufficiently stable, and accurate in its focussing movements, to be worthy of additions.

Most makers have a simple stand which corresponds to this one, and more or less closely resembles their better instruments in design. The cheapest form of 
students' microscope is non-inclinable, and fitted with a sliding-sleeve coarse adjustment (Fig. 9). The usefulness of these stands is very restricted when compared even with the last group, but for simple work with a vertical camera they answer very well. The instrument figured is by Leitz, and an excellent example of its kind.

Comparison of Stands.-If the microscopes described are compared, a difference is at once noticeable between the English stands, on the one hand, and the Continental and American stands, on the other. The English microscopes are supported on a tripod or claw foot, the axis on which the body swings being above the level of the stage, with the consequence that the balance between body and stage and substage is so good that the centre of gravity always falls well within the spread of the tripod, and the microscope is perfectly stable in a horizontal position. The Continental and American stands, on the other hand, are supported by a pillar rising from a horseshoe foot, and the point of support is below the stage. Consequently the microscope will not as a rule stand steadily by itself in a horizontal position, but requires to be clamped down to the table or otherwise supported.

Another difference is found in the substage mechanism, as is obvious on comparing Figs. 15 and 16 with 17 and 18 . There is more room between the stage and the table in the English patterns, and the condenser is carried in a ring, which is focussed by rack and pinion, and bears two or three screws to centre the optical part. The Iris diaphragm forms part of the condenser mount, and oblique light is obtained by dropping patch stops into a stop-carrier, also fitted into the mount. In the typical Continental stands, as shown in Figs. 15 and 16, the condensing system is more compact. The sleeve carrying the condenser is not usually fitted with centring screws, and the Iris diaphragm is carried on 
an independent swinging arm attached to the same support that carries the condenser sleeve. It may be rotated and moved across the condenser by rack and pinion to give oblique illumination. There is also a ring attached to carry patch stops, and frequently there is an Iris diaphragm above the condenser, which may be used in the stage when the condenser is withdrawn.

Too much stress must not be laid on the distinction here drawn between English and Continental practice, as many English makers have adopted the Continental pattern, in response to the demand for that style. The excellent series of "London" microscopes made by Beck are fitted with a horseshoe foot, and the Continental type of substage and foot has been adopted on Ross' Standard Microscopes. Leitz, on the other hand, is giving a claw tripod to some of his stands.

The chief points for and against each pattern may, however, be broadly stated as follows :-

Stability. - The greater stability given by the tripod foot and high axis is now generally recognised, and the horseshoe is being increased in size or altered in shape to approach it in that respect, but the small claw foot adopted by some makers does not give the stability of the original, on account of the lower point of support of the limb.

Compactness.-The horseshoe foot is superior in this respect, and permits readier access to the substage mechanism than the large tripod. On this account many workers prefer it for visual work, when the microscope is not often used in a horizontal position. The claw tripod shown in Figs. 17 and 18, is, however, almost as convenient as the horseshoe, and has more or less superseded the original wide-spreading pattern.

$\because$ Substage Mechanism.-The chief disadvantage of the Continental pattern lies in the absence of centring 
mechanism for the substage, and its smaller adaptability to a wide range of condensers, but centring screws are now added to the condenser rings of a few stands of this pattern, or to the condenser mount itself, or, as an alternative, to the nose-piece.

The diaphragm with rack-and-pinion movements permits of a more complete control over the illumination than is usually obtained by the English substage, but it increases the complication of the fittings, and the movement is not often required.

On the whole, the writers prefer the English pattern, with its greater stability and centring substage arrangements. These features are strong points in the design of the "Premier" microscope, which also includes the more complete illuminating device of the rackwork substage diaphragm, so combining the advantages of both types. 


\section{CHAPTER III}

\section{OBJECTIVES AND EYEPIECES}

In order to make this chapter as helpful as possible in the selection of objectives and eyepieces, a brief account is given of the main defects inherent in a simple lens, and of the methods adopted to correct them. Several of the more intricate errors are either entirely passed over or merely mentioned, as a discussion of them, and of the mathematical laws which govern their correction, is neither essential to a realisation of the advantages of

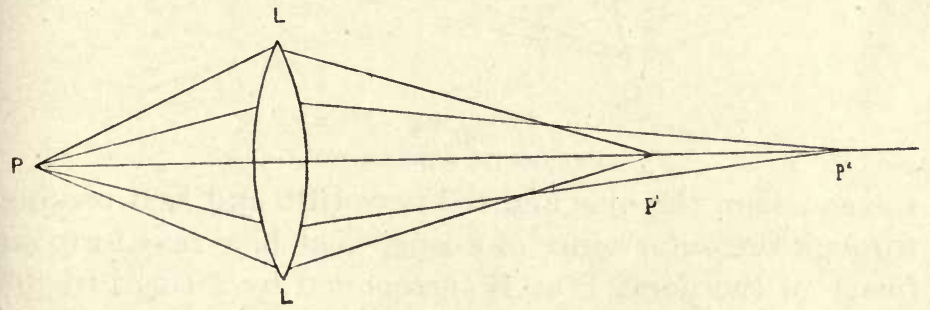

Fig. 19

SPHERICAL ABERRATION

one type of objective over another, nor to an intelligent application of the principles underlying the various methods and devices employed in photomicrography to overcome them. Many of the better-known optical terms are used without definition, and a knowledge is assumed of the general principles of refraction and of the formation of the solar spectrum.

Spherical Aberration.-Rays of monochromatic light from a point $\mathrm{P}$ incident upon different parts of the surface of a simple lens, LL, Fig. 19, are refracted in such 
a way that, although roughly brought to a focus after emerging, they do not actually pass through one single point. Those rays which pass through an annular zone near the circumference of the lens are focussed to a point on the axis nearer the lens than rays passing through a more central zone. The resulting deviation from an exact focus is called Spherical Aberration, and prevents the formation of a sharp image of the point $P$.

Chromatic Aberration is due to the difference of refrangibility of the different coloured rays which make up white light. Fig. 20 represents, in an exaggerated way,

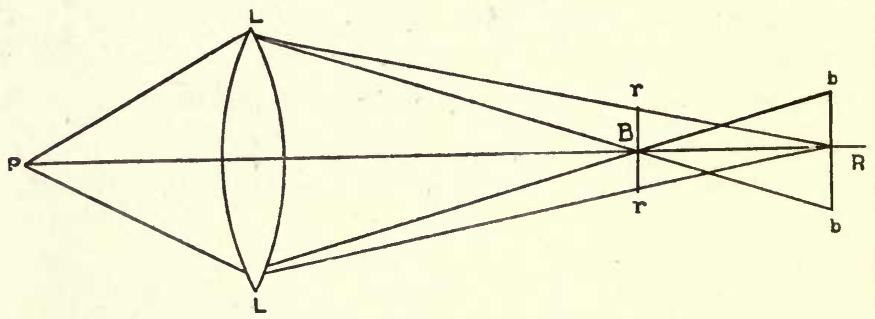

Fig. 20

CHROMATIC ABERRATION

the course of the blue and red rays $\mathrm{LBb}$ and $\mathrm{LrR}$ passing through the outer zones of a lens. The blue rays form an image of the point $\mathrm{P}$ at $\mathrm{B}$ surrounded by a ring of light formed by the red rays, and similarly the red rays form an image at $R$ surrounded by a blue ring $b b$. The other components of white light form images between $\mathrm{B}$ and $\mathrm{R}$. The amount of deviation suffered by any ray depends on the material of the lens, on its shape, and on the wavelength of the light in question.

Figs. 19 and 20 indicate that a single lens does not give a sharp image of a point, and further difficulties arise in photomicrography from the difference in luminosity and actinic power of the rays of different colour. The yellow-green rays are the brightest, and the image they form is that most accurately focussed on the 



\section{Low-power Photomicrography.}

Transverse Segtion through the Flower Bud of Salsify $\times 9$, stained with Borax carmine.

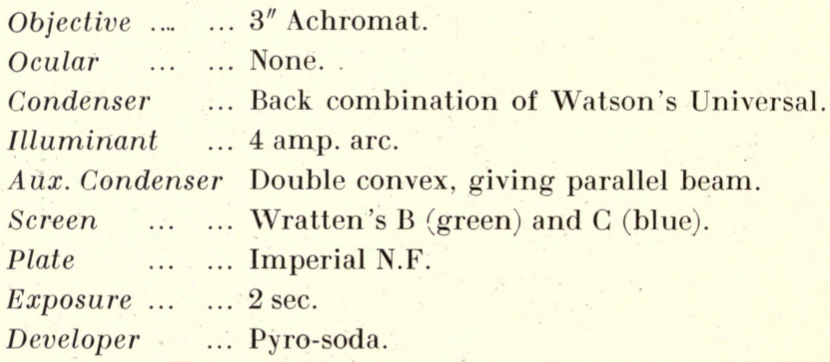

The section shows the structure of the inflorescence of the Compositæ, consisting of a number of individual florets, surrounded by an involucre of bracts.

An example of low power photomicrography, in which the use of the back combination of an achromatic condenser is to be noted as the illuminator employed with an ordinary $3^{\prime \prime}$ achromat. Good definition and contrast are ensured by using monochromatic light of wave length 4600 to 5100. The objective has sufficient covering power and flatness of field for an object of this type, but a micro-anastigmat is generally to be preferred. 


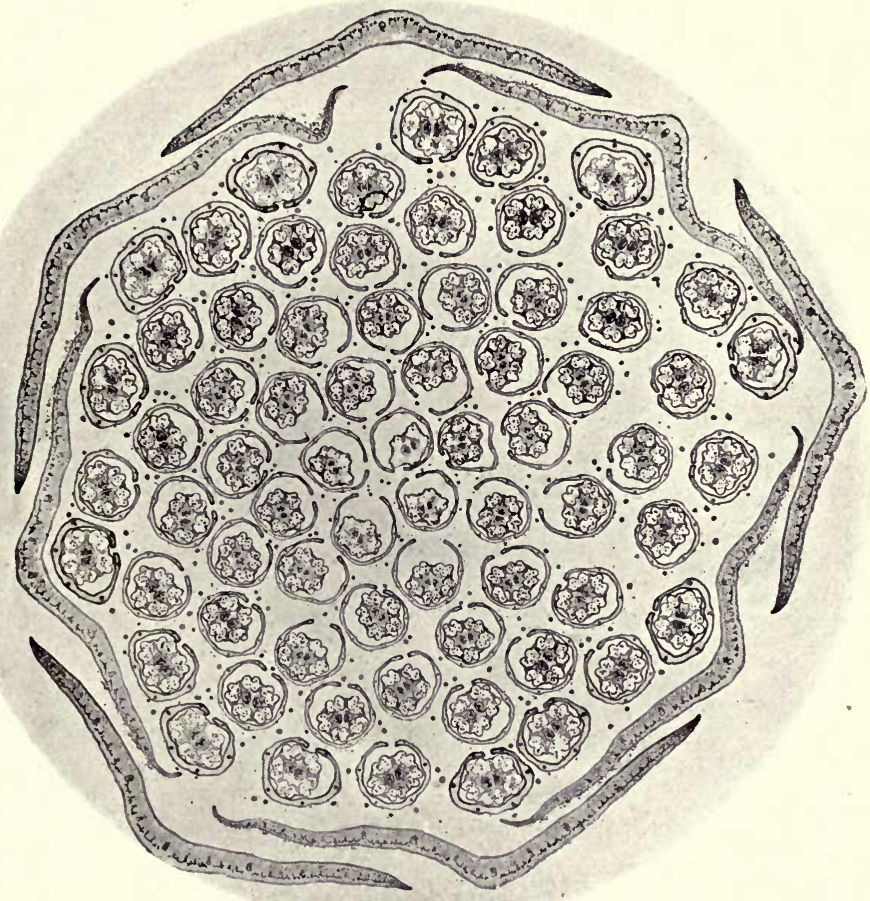






\section{Low-power Photomicrography.}

Transverse Section through the Petiole of Cycas revoluta $\times 10$

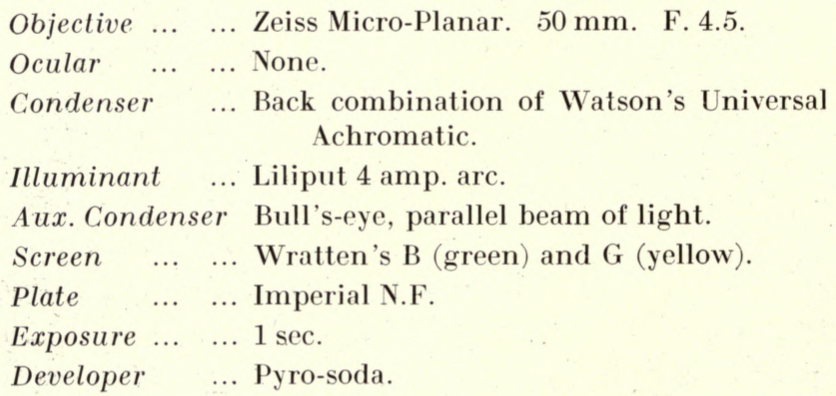

Section stained with Hæmatoxylin and Saffranin. Lignified tissue stained red, unlignified blue. Process given on page 261.

This is an example of low-power photomicrography for which the use of a planar lens is very desirable in order to obtain a flat field. The screens B and $\mathrm{G}$, together giving approximately monochromatic light of wave length 5100-6000, were used on account of the colour of the section, and also because their transmission corresponds with the colour with which the lens works to the best advantage. 


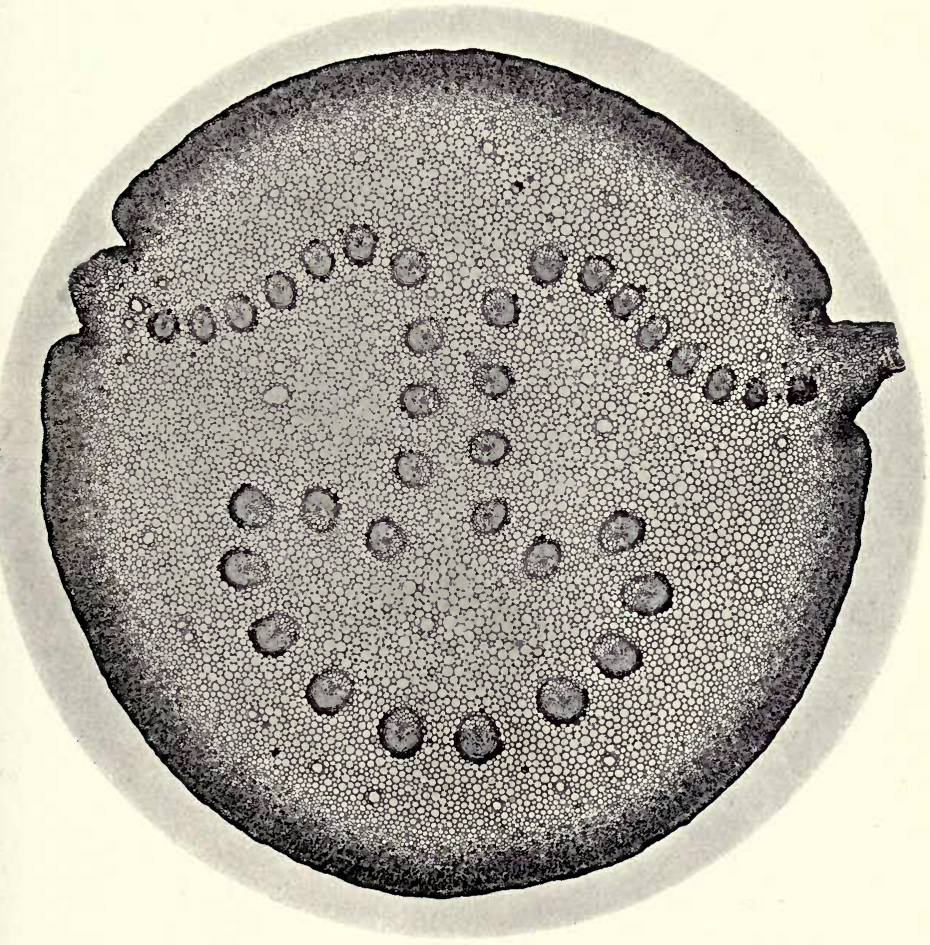



ground glass; the images formed by other rays would not be in accurate focus at the same time, but they are less luminous and may not detract very much from the sharpness of the composite image on the ground-glass. On the other hand, the effect produced by the different coloured images on a photographic plate is, under ordinary circumstances, quite different from that produced on the eye. The blue rays are the most actinic, and consequently the indistinct image they form, though not noticed when focussing, is registered more easily by the plate than the sharp yellow-green one, and gives a fuzzy photograph.

The Correction of Lenses.-There is a wide selection of glasses suitable for lens-making, each with its own special physical properties-Refractive Index, Dispersive Power, etc. - and a lens in which the errors are more or less eliminated can be constructed by a proper combination of two or more simple lenses whose shapes and materials have been carefully calculated and selected so that their errors balance.

The Refractive Index of any medium is the sine of the angle made by a ray of light in vacuo (or practically in air) incident upon its surface, and the perpendicular to that surface, divided by the sine of the angle made by the same ray to the perpendicular, after it has passed into that medium. It will make the methods of correction clearer if figures are given for a typical crown and flint glass :-

Ref. Index of Crown Ref. Index of Flint Glass. Glass.

\begin{tabular}{c|l|l}
\hline For a red ray " C" & 1.515 & 1.710 \\
" yellow "D " & 1.518 & 1.717 \\
" blue-green "F" & 1.524 & 1.735
\end{tabular}

The Dispersive Power measures the difference between the refractions of two selected rays compared with that of 
a definite ray, the Sodium line "D" for example; for the above glasses it is

$$
\begin{array}{ll}
\text { Crown } & \frac{1.524-1.515}{1.518-1}=.017 \\
\text { Flint } & \frac{1.735-1.710}{1.717-1}=.035
\end{array}
$$

In order to make a corrected lens from the glasses whose constants have been given, a double convex lens of crown glass is cemented to a negative lens of flint, in the way shown in the sections of lenses (Figs. 34 to 36). The crown glass lens is of higher power than the flint, so that the combination still functions as a positive lens, and the two components are so selected as to give the desired power to the combination. The dispersive power of flint glass is greater than that of crown, so it is possible to find a positive crown and a lower-power negative flint whose dispersions, for two selected colours, will balance each other. As the figures given above indicate, the differences of Refractive Index of different pairs of colours are not proportional in the two glasses, and it is not possible to bring more than two colours to a common focus by their combination.

The Spherical error of a simple lens is comparatively small when only a narrow pencil of light through a central zone is employed, but as microscope objectives have to utilise wide-angled pencils over their entire surface, spherical aberration becomes of the greatest importance. Lenses of the same power can be made of different shapes, and the spherical aberrations depend on their shapes, so it is possible to select a powerful positive lens with small aberrations, and a less powerful negative lens of greater relative aberration, which will form a corrected lens when combined. As the different coloured rays are differently refracted, it is necessary to 
select a definite colour for which this correction is made; a yellow-green from the brightest part of the spectrum is usually chosen.

The possibility of the simultaneous elimination of chromatic and spherical aberrations arises from the circumstance that the first is corrected by a careful adjustment of the foci of positive and negative elements whose shapes are immaterial. The second is eliminated by adjusting the shapes of the lenses whose foci have been predetermined. But in the best lenses made from ordinary crown and flint glass the colours, other than those specially selected for correction, still deviate from exact focus, and form the "Secondary Spectrum," focussing together in pairs further away from the lens. Accurate spherical correction, even for the selected colour, is not usually achieved over the entire surface of the lens, and rays from an intermediate zone do not focus strictly with the rays passing through central and peripheral zones. The residual "zonal aberration" is most difficult to overcome. For these reasons the formation of Colour Fringes is still noticeable in the examination of certain objects, particularly when oblique light is used.

This is necessarily a very incomplete statement of the defects and corrections of lenses, but the outline given is sufficient for an elementary explanation of the differences found in objectives and their bearing on photomicrography. A discussion of the other difficulties which have to be overcome-for instance, those involved in satisfying the "sine law" and preventing "coma" would introduce unnecessary complication, and reference for that should be made to Mr. Conrad Beck's Cantor Lectures, The Theory of the Microscope, to Dr. Spitta's Microscopy, or works on Optics where a fuller survey of the subjects will be found. The adjustment of the different elements that go to make up a corrected lens 
is a mathematical problem of great delicacy, involving a very careful selection of the materials and an accurate computation of their curvatures, powers, and shapes, so as to eliminate all the errors simultaneously, and full correction is only attained by the combination of as many as six or even ten lenses in one objective.

Objectives.-A lens corrected for spherical error is called "Aplanatic," and for chromatic error, "Achromatic." The objectives generally used are divided into two classes, according to the way in which these corrections are carried out, called respectively, "Achromatic" and "A pochromatic." Messrs. Zeiss have recently added a third group for use with ultra-violet light, to which they give the name "Monochromatic." The newer types of Achromatic objectives are such an advance over their predecessors that they are almost worthy of classification in a special group between the Achromatic and Apochromatic and, in fact, several makers call them "Semi-Apochromatic."

Achromatic Objectives are chromatically corrected in such a way that two colours come to the same focus, while the most accurate spherical correction is confined to rays of one selected colour. They show more or less zonal aberration.

As these objectives are primarily intended for visual work, the colour selected for the most accurate spherical correction is usually a yellowish green from the most luminous part of the spectrum. If matters are so arranged that the other images are practically coincident with that formed by the preferred colour, the lens will give very good photomicrographs, and good modern achromats fulfil this condition very well, but an absolutely sharp negative cannot be obtained when using white light, although the error is frequently small enough to be unimportant except for the most exacting work; on the other hand, many older lenses are very deficient in this respect, unless they were specially corrected for 
photography rather than for visual work, an arrangement still advised by certain makers, but becoming more and more unnecessary as improvements in lens-making are made. With older lenses, not specially corrected to focus simultaneously the actinic and visually brightest rays, it is frequently impossible to get a sharp photomicrograph unless monochromatic light is used, except by moving the back of the camera, after focussing, by a distance that previous experiments have shown to be necessary. This is obviously a most unsatisfactory method of working and should not be needed with modern objectives, and any lens that does not give a reasonably sharp photograph after careful focussing in ordinary light should be rejected. Colour screens provide a remedy for this state of things by intercepting rays other than those for which the objective is corrected before they reach the lens (see p. 161).

Apochromatic Objectives.-It has been mentioned that perfect correction of objectives is unattainable by the combination of ordinary crown and flint glasses, and up to $\mathbf{1 8 8 6}$ the difficulties involved in the use of imperfectly corrected lenses were unavoidable. But about that time, as the result of extensive researches, Prof. Abbe and Messrs. Zeiss introduced a new series of objectives, in which they utilised a mineral called Fluorite and new glasses made at the Jena Glass Works. Substances not previously used in glass-making were included in the composition of the new glasses, Phosphoric and Boric oxides in place of Silica, for instance, and by that means materials of optical properties hitherto unattained were produced. A higher refractive index was found in conjunction with a lower dispersion, or vice versa, and a greater proportionality existed in the dispersion of the different parts of the spectrum. The objectives, made as a result of these discoveries, mark a very great advance on anything produced up to that time, and 
as their corrections are altogether superior to those found in achromatic objectives, Prof. Abbe introduced the term "Apochromatic" to distinguish them. Spherical aberration is corrected in them for two colours instead of one, as in achromatic objectives, and three colours are brought to a common focus instead of two, which so reduces the distance between the foci of the various parts of the spectrum that secondary spectrum is almost eliminated. These corrections can, moreover, be accurately made for all zones of the objective. Their construction is, however, more complex than that of achromats of corresponding power, and their cost very considerably higher.

Semi-Apochromatic and Fluorite Objectives.-Improvements in the corrections of achromatic objectives are continually being made, mainly by the introduction of the new glasses and fluorite in their construction, with a consequent recomputation of the components. Several makers call the lenses so produced "Semi-apochromatic," others use such terms as "Improved achromatic," and "Fluorite" to describe them, or give them a special name, such as "Holoscopic." They really are improved achromats, and have not the complete correction of the apochromats, but, on account of the high transparency of fluorite and the more perfect corrections made possible by the use of that mineral and the new glasses, these lenses approach very closely to apochromats in working properties, although only chromatically corrected for two colours, and yet, on account of their comparatively simple construction, they are produced at much lower prices than the more complicated apochromatic objectives.

Under-Correction of High-Power Objectives.-High-power objectives of wide aperture (p. 160), both apochromatic and achromatic, have an uncorrected front lens almost hemispherical in shape, and exhibit an outstanding defect 
known as "chromatic difference of magnification." The different coloured images are of varying size, the blue larger than the red, and consequently they do not unite to form a colourless final image. This is corrected by using oculars specially constructed to magnify the original red image more than the blue, and called "compensating oculars" (see p. 72). In order that these oculars may be used with the whole series of apochromatic objectives, the same under-correction is intentionally introduced into the lower powers.

Superiority of Apochromats. - The chief advantages of apochromats may be stated thus :-

1. Their corrections are so good, and the images they produce so perfect, that a high eyepiece can be used to further magnify them without showing defects.

An eyepiece magnifying the image produced by the objective 6 or 8 times is about the highest that can be used with an ordinary achromat; a good apochromat will stand 18 or even as much as 27 times without breaking down.

2. They can be given a greater numerical aperture (p. 160), and the aperture can be used to its limit, which is not usually the case with an achromat.

3 . They produce a more brilliant image than an achromat, and can therefore be used with higher eyepieces without an undue loss of brightness.

4. Their corrections are so good all over that the colour defect, introduced into the high powers by the hemispherical front lens, can be corrected by an eyepiece much more perfectly than is possible in an achromat.

5. They form a perfect image right to the edge of the field. This does not imply that the whole field can be simultaneously focussed. In fact, it is frequently less flat than "that of an achromat.

6. An object focussed by white light will still give 
a sharp image if a coloured light replaces the white without alteration of the fine adjustment. That is, an object can be focussed with white light, a colour screen then introduced, and a photomicrograph taken without further adjustment, which is, as a rule, not possible with achromats.

7. They are also very desirable for visual use, as they do not put such a strain on the accommodation of the eye, and can be used for longer periods without fatigue. A pochromats and Achromats compared for Photography.From what has been said, it will be gathered that the objectives par excellence for photomicrography are apochromats, and they should always be chosen if their cost does not prevent it. But the question naturally arises as to whether much is lost by substituting the less expensive achromats for them; and the results given by the modern achromatic objectives, and particularly those containing fluorite or described as semiapochromats, are so good, when used with suitable screens (Chapter IX), that the majority of workers need feel no reluctance in selecting them. At the same time, having once become accustomed to apochromats, either for visual or photographic purposes, one would very reluctantly go back to achromats. High-power achromats have usually one distinct advantage over corresponding apochromats, namely, a flatter field.

Testing Objectives.-Many important factors have to be considered when selecting the individual objectives. Their capabilities can only be determined with certainty by a very careful examination of test objects, and by actual use in photomicrography; but objectives are now so carefully made and tested that the claims of the maker as to their working properties will usually be found to be well sustained, and the probabilities are that the differences between similar objectives of different makers of repute, or of the same maker, can only be detected and their 
importance realised by expert workers. Consequently, it is always advisable for a beginner in microscopy to be guided by an experienced friend, or, failing that, to rely upon the maker, whose lenses he has decided to buy, to supply the best for his particular needs, and on no account to purchase lenses that do not bear the name of a well-known house, or old lenses, unless they have been carefully tested.

Test Objects.-There are certain objects which are generally employed as tests, and from the examination of which a skilled observer can judge of the performance of a lens. Photographs are given of a few such on Plates $9,34,41$ and 43 , and the points of importance are noted with each.

The value of such test objects depends not only on the quality of the mount, but more particularly on the observer's familiarity with them. For instance, although certain diatoms or the markings on the scales of Podura are perhaps the favourite test objects for high powers, many observers would use with greater success certain species of bacteria with which they are more familiar. Many of the defects, or otherwise, of objectives may be made out with considerable facility by means of the Abbe test-plate, which is a slide with six silvered cover-glass discs of certain definite thicknesses, or a strip of graduated thickness, cemented on to it. These are silvered on the under side and ruled with fine lines. The lines on the cover-glasses or strip should be examined, and the thickness of cover-glass determined for which the objective has been most accurately corrected (p. 69). When this has been found, the lines under it are examined with axial and oblique illumination, and if focussed for axial, should remain in focus, when oblique light is used, showing that the spherical correction of the lens is satisfactory. Chromatic aberrations, and the nature of the corrections embodied in the ob- 
jective, are evidenced by the appearance of the colour fringes on either side of the lines under suitable conditions of illumination. Apochromatic objectives, when used with compensating oculars, give practically no colour, while a good achromat gives only slight fringes.

Finally, it is practically impossible to pick out the objectives of any one maker as better than those of others. The output of any of those whose microscopes have been mentioned can be relied upon as being of the highest class. The following have been used to make the photomicrographs from which the illustrations are taken :-

Achromatic Objectives-

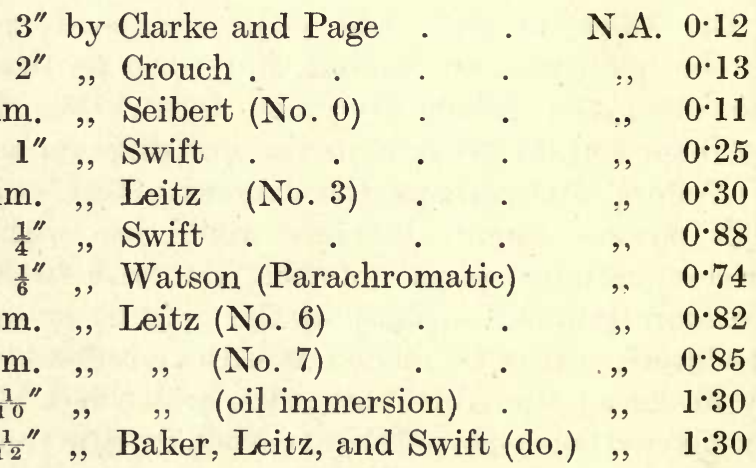

Apochromatic Objectives-

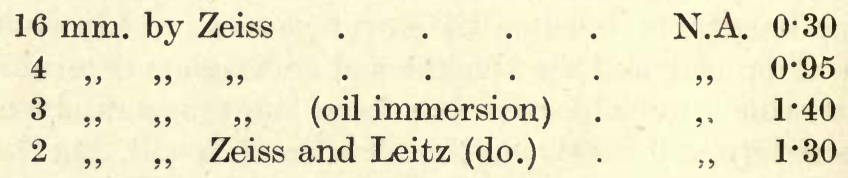

Micro-Anastigmats-

$25 \& 50 \mathrm{~mm}$. Micro-planars by Zeiss

$75 \mathrm{~mm}$.

Ross

$3^{\prime \prime} \cdot$ Anastigmat (No. 0) by Aldis 
Photographic Lenses -

7" Goerz Anastigmat by Ross

$5 \frac{1}{2}$ " Homocentric " Ross

Dallmeyer's “Adon " Telephoto Lens

Properties of Objectives.-The following factors must all be taken into account when selecting objectives, and many of the points can be made out by a careful examination of one or other test object; but it must be borne in mind that it is usually necessary to make a compromise when constructing a lens with a view to general work. Some good property, almost essential for one purpose, must be sacrificed to a slight degreé to gain others more necessary for other purposes; instances of this are mentioned.

Focal Length and Power of Objectives.-Objectives are designated either by their "equivalent focal lengths" or by certain arbitrary letters or numbers, and the term equivalent focal length calls for some explanation. It is possible to imagine the substitution of an "equivalent" simple lens for any compound lens, so that the simple lens will have the same magnifying effect as the other; but the path of the rays through the compound lens can only be reproduced by the simple lens if it is further imagined to exist in two different planes at once. In the first plane it may be supposed to receive the rays from the object, in the second to transmit them. The focal length of this simple lens is taken as the "equivalent focal length" of the objective, and the two planes in which its focus is supposed to exist are the upper and lower focal planes of the objective. The power of an objective varies inversely as its equivalent focal length.

Magnification always refers to increase in linear dimensions, and is more fully dealt with in connection with the use of objectives and eyepieces together. An 
estimate can be made of the magnification produced in the photomicrograph of any object, when the plate is placed at a given distance (say, $10^{\prime \prime}$, or $250 \mathrm{~mm}$.) from the shoulder of the objective, by dividing this distance

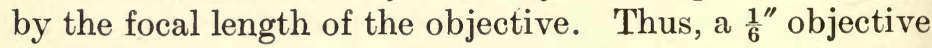
will give an amplification of $10 \div \frac{1}{6}=60$ times, and a $2 \mathrm{~mm}$. will give $250 \div 2=125$ times under these conditions when used without an eyepiece.

Numerical Aperture.-Most objectives are marked by a figure preceded by N.A., and this figure is of the greatest importance, as it indicates the properties of the lens in a way unattainable even by long description. It is found that the wider the cone formed by the extreme rays proceeding from a point which the objective can utilise, the better, and that the perfection of the image depends on the amount of light so gathered. The number of degrees in the angle of the cone was used at one time as a measure of the "aperture" of an objective, but it did not give any direct comparison of the properties of dry and immersion objectives. Prof. Abbe consequently substituted for it the sine of half the angle of aperture multiplied by the refractive index of the medium (air, oil, etc.) in which the objective works, and called this value "numerical aperture," so that-

$$
\text { N.A. }=n \sin \alpha
$$

where $\alpha=$ half the angle of aperture,

and $n=$ the refractive index of the immersion fluid.

The following properties depend on the width of the cone of light gathered by the objective, and of them the "numerical aperture" forms a measure.

Resolving Power.-Varies directly as the N.A.

Brightness of Image.-Varies as the square of the N.A. for any given magnification.

The following properties generally become less as the N.A. increases :- 
Penetration.

Flatness of Field.

Working Distance.

Some of these require fuller discussion, as they are of great importance in photomicrography (see pp. 255-257).

Resolving Power is the ability to display, distinctly and separately, lines or dots which are very close together. This property is equally as important as magnification, for without adequate resolution no increase of magnification will make fine detail distinct. Detail must first be resolved into its component parts, and then amplified until the eye can see it well, and this involves the necessity of giving high-power objectives greater resolving power than low ones, so that their increased magnification may be accompanied by the ability to distinguish finer detail.

Resolution is not only dependent on the N.A. of the objective, but also increases as the wave length of the light employed decreases, and this is equally true of the finest apochromat as of an achromat. To achieve the highest resolution of which either is capable in ordinary photomicrography, monochromatic blue or violet light is used ; but green often gives appreciably better results with achromatic objectives, as it is nearer the wave length with which the objective performs to the best advantage. The greater ease with which focussing is carried out in green over blue light must also not be lost sight of. Resolution is impossible without a previous elimination of the spherical and chromatic errors, and this also is a most important function of monochromatic light.

The greatest number of lines to an inch, or millimetre, that a lens can resolve by oblique light of given wave length is twice the numerical aperture divided by the wave length in fractions of an inch or millimetre :- 


\section{Limiting resolving power $=\frac{2 \mathrm{~N} . \mathrm{A}}{\lambda}$}

Equivalent to N.A. $\times 96,410$ lines per inch, for white light, $\lambda=\cdot 5269 \mu$ or 5269 A.U. (see p. 158 for definition of units).

An objective illuminated by a $\frac{3^{\prime \prime}}{4}$ solid axial cone should resolve-

\section{N.A. $\times 70,000$ lines per inch for white light.}

A normal eye can resolve lines separated by about $\frac{1}{50} 0^{\prime \prime}$ at $10^{\prime \prime}$. Consequently, if a microscope magnifies 100 times, lines $\frac{1}{25,000 "}$ apart ought to be resolved by the objective, so that they may appear as $\frac{1}{2} \frac{1}{50}$ "apart in the image. A $1^{\prime \prime}$ objective used with a $\times 10$ eyepiece gives 100 magnification, and, if full resolution is to be obtained under these conditions, the objective should be capable of resolving 25,000 lines to the inch. If it is given a N.A. of 0.26 , it will be able to do this with an oblique beam of white light.

Under the same conditions a $\frac{1}{4}$ " objective should magnify 400 times with the $\times 10$ ocular, and be capable of resolving 100,000 lines to the inch, requiring a N.A. four times as great, or N.A. 1.04. That is, to obtain the utmost resolving power appreciable by a normal eye, an objective should be given a N.A. of 0.26 for every. 100 magnifications it gives with a $\times 10$ ocular, selected as about the highest with which an achromat can perform to advantage.

The following table gives the limiting resolution of objectives of various numerical apertures calculated from the formula $\frac{2 \mathrm{~N} . \mathrm{A} \text {. }}{\lambda}$ for orange, green, and violet light, and also includes values for (N.A.) ${ }^{2}$, which measures the brightness of the image, and is an important factor in the determination of exposures, and for $\frac{1}{\text { N.A. }}$, which 
gives an approximation to the depth of focus of an objective.

\section{Limiting Resolution}

\begin{tabular}{|c|c|c|c|c|c|c|}
\hline Objective. & N.A. & $\begin{array}{l}\text { Yellow line D } \\
\lambda=5896 \text { A.U. }\end{array}$ & $\begin{array}{l}\text { Green line } \mathrm{E} \\
\lambda=5269 \text { A.U. }\end{array}$ & $\begin{array}{l}\text { Violet line H } \gamma \\
\lambda=4341 \text { A.U. }\end{array}$ & (N.A.) $)^{2}$ & $\frac{1}{\text { N.A. }}$ \\
\hline $1 \frac{1}{2}$ & $\cdot 10$ & 8,617 & 9,641 & 11,699 & $\cdot 01$ & $10 \cdot 0$ \\
\hline$\frac{2}{3}$ & $\cdot 25$ & 21,542 & 24,103 & 29,247 & $\cdot 063$ & $4 \cdot 0$ \\
\hline$\frac{1}{3}$ & $\cdot 6$ & 51,700 & 57,846 & 70,193 & $\cdot 36$ & 1.67 \\
\hline$\frac{1}{6}$ & $\cdot 9$ & 77,550 & 86,769 & 105,289 & $\cdot 81$ & $1 \cdot 11$ \\
\hline Oil immersion $\frac{1}{12}$ & $1 \cdot 4$ & 120,638 & 134,974 & 163,783 & $1 \cdot 96$ & $\cdot 71$ \\
\hline
\end{tabular}

The highest resolution as yet obtained is achieved by the use of ultra-violet light of a wave length of 2750 or 2800 A.U. But the special properties of these rays, involving the use of quartz lenses instead of glass, which is opaque to ultra-violet rays, make the process so expensive, that a description of the apparatus, which is made by Messrs. Zeiss, is not given in this book.

Brightness of Image. - This property requires no further explanation, but it is of importance, as it governs exposure. The superiority in this respect of apochromatic and fluorite lenses over achromats is very marked, and is a great point in their favour, particularly with high powers which need a very good light or long exposure.

Penetration is the power of an objective to bring points in various planes to a sufficiently exact focus at the same time, and is of importance when photographing an object of any thickness. It decreases as the square of the equivalent focal length, the numerical apertures being the same. Thus, a $4 \mathrm{~mm}$. objective has four times the depth of focus of a $2 \mathrm{~mm}$. of the same numerical aperture. 
The image of a point formed by a lens is a disc of very small size. The image is in focus when that disc or circle of confusion is less than $\frac{1}{250} "$ in diameter, in which case it appears as a point to a normal eye at $10^{\prime \prime}$ distance. The condition under which the images formed in one plane of two points in different planes shall appear sharp, is that they are both smaller than the greatest permissible circle of confusion. If one point is in focus the size of the image of the other depends not only on its vertical distance from the first, but on the breadth of the cone of light producing the image, that is, on the N.A. of the objective, and, of two lenses of the same power, that with the lower N.A. will have the greater penetration. The best means of obtaining greater depth of focus, without altering magnification, is to substitute a lens of lower N.A. of the same power, or a lower-power lens combined with greater camera extension. There is in either case a loss of resolution, but resolution, as a rule, is not required at the same time as penetration.

Another method for increasing penetration, and without a change of objective, is to reduce its aperture by a diaphragm above it, or by restricting the aperture of the condenser; as the iris is closed points further and further away in vertical direction come progressively into sufficiently accurate focus. But there is a limit to this use of the diaphragm, as diffraction effects are also produced (p. 121).

An increase of the refractive index of the medium in which an object is mounted helps the objective to simultaneously focus more than one plane. As an illustration of this the well-known experiment, whereby a stone invisible in an empty jug becomes visible when water is poured in, may be cited. In a similar way, supposing the bottom layer of an object mounted dry is slightly out of focus when its top is focussed, it may be brought into 



\section{Low-power Photomicrography.}

A Section of Human Skin $\times 12$

$$
\begin{array}{llll}
\text { Objective } & \ldots & \ldots & \text { Zeiss } \mathrm{A}_{2} . \quad 37 \mathrm{~mm} . \\
\text { Ocular } & \ldots & \ldots & \text { None. } \\
\text { Plate } & \ldots & \ldots & \text { Ilford ordinary. } \\
\text { Illuminant } & \ldots & \text { Incandescent electric, } 16 \text { c.p., in frosted globe. } \\
\text { Exposure } & \ldots & \ldots & 30^{\prime \prime} .
\end{array}
$$

This photomicrograph was taken with the simplest apparatus; the definition and detail are quite satisfactory for a low-power photograph of a histological section, and indicate that the most limited resources are frequently adequate. No condenser or colour screen was used, the illuminant was of the crudest description, and the objective a simple achromatic lens.

Sweat ducts are visible in the section, passing through the dermis, with spiral portions in the epidermis.

\section{Medium-power Photomicrography.}

B Cylindrospermum macrospermum $\times 250$

Objective ... ... Leitz No. 3. $16 \mathrm{~mm}$.

Ocular .... ... Watson's B.

Condenser ... Watson's Universal, front lens removed.

Illuminant ... Liliput 4 amp. arc.

Aux. Condenser Biconvex lens, parallel light.

Plate $\quad \ldots . .$. Wellington Anti-Screen.

Exposure $\ldots \quad \ldots 2^{\prime \prime}$.

Cylindrospermum is one of the blue-green algæ forming slimy gelatinous masses on stones; it was photographed in the living state, mounted in a drop of water. 


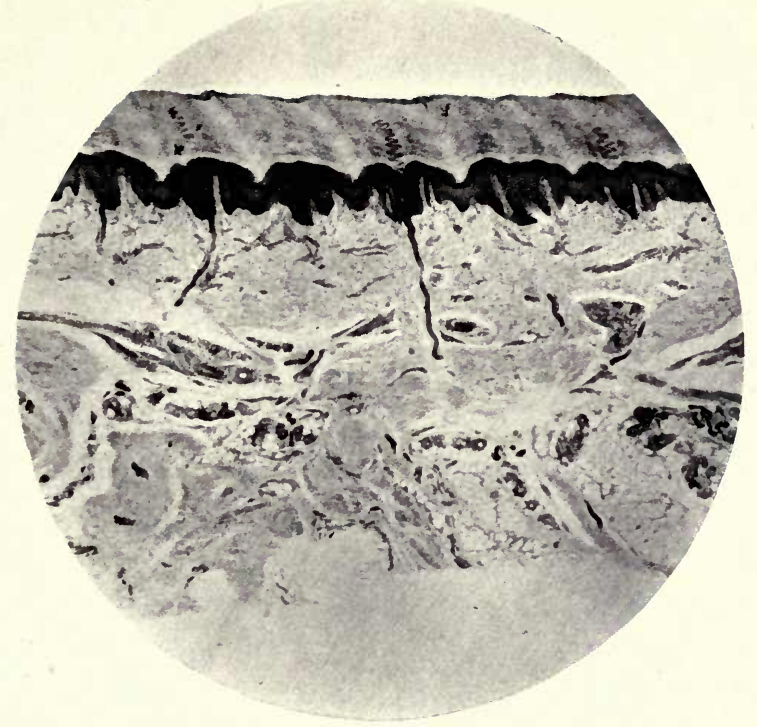

A

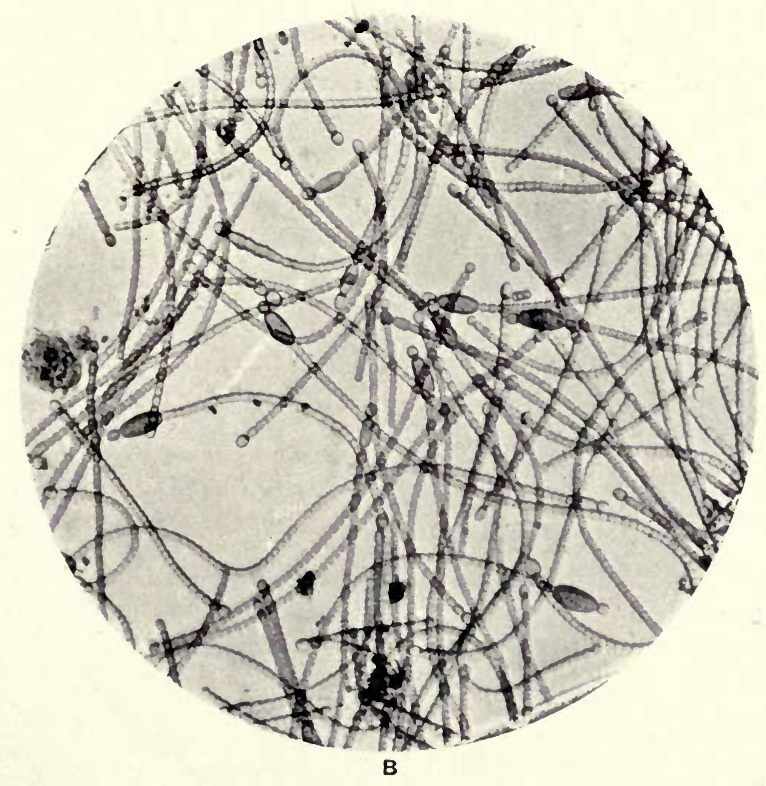



focus all over by mounting it in Canada balsam, or by immersing it in water. The latter is a device sometimes useful when photographing fairly large objects.

Flatness of Field.-A "flat field" is one in which objects at the centre and periphery are simultaneously in focus, and it is very desirable that low-power objectives should simultaneously focus every part of an object that they cover. At present it is not possible to combine this property with perfect corrections in an objective of the ordinary construction, and when a photomicrograph of a large surface is required it is best to use one of the small photographic lenses specially constructed for that purpose, and described on page 79. High-power objectives are usually required to give an image of a small part of the field with perfect detail, and great flatness of field cannot be expected under these circumstances from an objective of higher power than $\frac{1}{4}^{\prime \prime}$ or $\frac{1}{6}$. . The roundness of field of high-power apochromats is very noticeable, but the real test of a high-power objective is found in the perfection of definition of an object focussed at the edge of the field, which should be as good as when focussed at the centre.

Working Distance is the distance between the front lens of the objective and the object, or cover-glass, when in focus. It must not be confused with focal length, which is not measured from the front lens, but from the lower equivalent plane of the objective, which may be above or below the front lens, according to the construction of the combination. Lenses of the same power vary considerably in working distance, which becomes important in those of $\frac{1}{6}$ " and upwards, particularly when thick mounts have to be examined. It is also much easier to focus rapidly a lens of long-working distance than one which involves very near approach to the object, and consequent danger of fracture of the coverglass. 
As an example of the variation of working distance with N.A., three lenses of approximately the same focal length made by Zeiss may be instanced:-

The $4 \mathrm{~mm}$. apochromat has a N.A. of 0.95 and a working distance of $0.2 \mathrm{~mm}$.

The $4.3 \mathrm{~mm}$. achromat has a N.A. of 0.85 and a working distance of $0.4 \mathrm{~mm}$.

The $4.2 \mathrm{~mm}$. achromat has a N.A. of 0.65 and a working distance of $0.6 \mathrm{~mm}$.

N.A. and the Power of Objectives.-Although an objective is judged by the definition it gives, which depends on the perfection of its corrections, on its resolving power, and on the brightness of the image formed, a lens of high N.A. is not always the most satisfactory. Penetration, flatness of field, and working distance must all be taken into account, and the special requirements considered; and for many purposes it is best to make a compromise and select one with a moderate N.A.

N.A. of Low-Power Objectives.-In low-power work the chief considerations are usually flatness of field and penetration. Resolution may generally be neglected, so a low N.A. is all that is required.

N.A. of Medium-Power Objectives.-The selection of an aperture suitable to the work in hand is of considerable importance with medium-power objectives. To take the Zeiss $4 \mathrm{~mm}$. objectives given above, as examples, the resolving power of the apochromat and of the achromat of N.A. $0 \cdot 85$ are as great as can be desired with dry lenses of each class, but their penetration and working distance are notably less than those of the achromat of N.A. $0 \cdot 65$, and it is well to sacrifice some of the resolving power and be content with a N.A. of 0.65 when working distance or penetration is important.

N.A. of High-Power Objectives.-The maximum resolution possible must be achieved by high-power objectives, 
and, as a rule, great working distance and penetration are not required. For the finest resolution of diatoms, $\frac{1}{12} "$ oil immersion apochromats with a N.A. of $\mathbf{1 . 4}$ can be obtained, and Messrs. Powell and Lealand make a $\frac{1}{10}$ " of N.A. $1 \cdot 5$, but objectives of such high aperture are so easily damaged, owing to the difficulty of holding the hyperhemispherical front lens firmly in the mount without cutting down its aperture, that an objective of N.A. 1·2 or 1.3 is to be preferred for general work. The N.A. usually associated with objectives of various powers is given in the table on page 81 .

Oil-Immersion Lenses. - The formula N.A. $=\mathrm{n} \sin \alpha$ indicates that with dry lenses the greatest possible numerical aperture is $\mathbf{1}$, as $\mathbf{n}$ for air is $\mathbf{l}$, and the greatest value of $\sin \alpha$ is 1 . The maximum reached in practice is about 0.95 , for instance, in the Zeiss $4 \mathrm{~mm}$. apochromat. At the same time the formula points to a possible method of increasing N.A. by increasing the value of $n$, and this is done by making the objective work in a medium of higher refractive index than air. An objective so used is called an oil- or water-immersion lens, according to the medium employed. Cedar oil is the most usual immersion fluid, as its index of refraction is practically equal to that of the cover-glass, namely, 1.515 , on which account an oil-immersion objective is spoken of as a homogeneous immersion lens. Water and glycerine are also used, but as their indices of refraction are less than that of cedar oil they are not so efficient, and Messrs. Zeiss have introduced the use of Napthalene Monobromide (index of ref. 1.65) for use with some lenses of extreme aperture, but it is necessary to use special slides and cover-slips made of glass having the same refractive index, otherwise the great N.A. cannot be utilised. These are not much in use. The following diagram explains the superiority of oil-immersion over dry lenses, by indicating how they gather more light from an object than similar dry ones. 
There is also much less reflection at the lens surface when the light passes through oil.

The Effect of Oil Immersion on Numerical Aperture.-

lens of objective

Immersion Fluid

Cover-glass

Object Slide

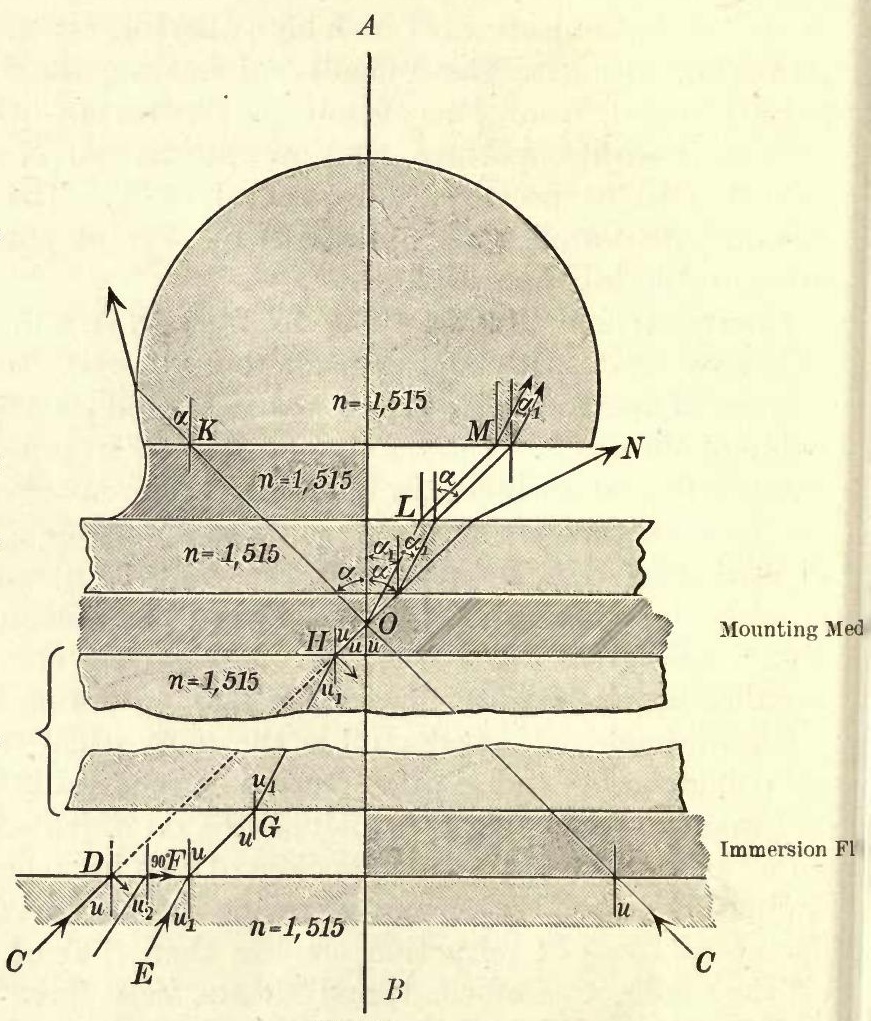

Fig. 21

COMPAIISON OF OII,-IMMERSION AND DRY SYSTEMS

The aperture of the objective and its light-gathering power depend on the obliquity of the rays which can enter it. In the diagram OK represents the extreme ray. The effect of the condenser on the illumination of the object and on the N.A. of the objective will be considered 
later on, in reference to the part of the diagram below $O$. In the figure the mounting medium, the glasses, and the immersion fluid are all represented as of the same refractive index 1.515 , that of cedar oil, and consequently the ray OK passes through the system without any refraction. $\mathrm{AOK}=\alpha$ is half the angle of aperture of the objective whose N.A. ${ }^{\text {(oil) }}=\mathrm{n} \sin \alpha$, where $\mathrm{n}=1.515$.

Now suppose the objective is used dry, but the object still mounted in the same medium as shown on the righthand side of Fig. 21. A ray leaving $\mathrm{O}$ at the angle $\alpha$ to the perpendicular no longer enters the objective, but is refracted at the surface of the cover-glass to N. Suppose $\alpha_{1}$ is the greatest angle at which a ray can leave $O$ and still be received by the objective. It will be refracted at $\mathrm{L}$, forming there an angle $\alpha$ to the perpendicular, and the numerical aperture of the dry lens is given by

$$
\text { (N.A.) }^{\mathrm{dry}}=1 \sin a \therefore \frac{\text { (N.A. }^{\mathrm{dry}}}{\text { (N.A.) }^{\text {oil }}}=\frac{\sin \alpha}{\mathrm{n} \sin \alpha}=\frac{1}{\mathrm{n}}
$$

That is, the N.A. of the objective is increased $\mathrm{n}$ times when immersion oil is used.

It follows that much higher resolution can be achieved by an immersion over a dry lens of the same magnification, whilst at the same time the image is much brighter. For this reason the lenses of higher power than $~_{8}{ }^{\prime \prime}$ are now almost universally made for oil immersion, and have a large N.A. of 1.2 to 1.4 . Their increased resolving power over dry lenses of similar magnification is very marked, and the exposures when they are used for photomicrography may even be less than those required by dry lenses of half their power.

Correction for Cover-Glass Thickness.-Owing to refraction in the cover-glass the optician has to. compute his objectives for a standard thickness of cover-glass, giving a definite amount of refraction. $0 \cdot 18 \mathrm{~mm}$. is that usually chosen and marked on the objective. For low powers 
any moderate deviation from this thickness will not have much effect. But when using high-power dry lenses it is necessary in critical work either to be careful to use cover-glasses of the specified thickness, or to make an adjustment for unavoidable differences found in various mounts. Correction collars are fitted to many dry objectives of $\frac{1}{4}$ " and upwards, and the adjustment is made by a slight alteration of the distance between the component lenses of the objective. Alteration of the tube-length of the microscope may also be employed to obtain a suitable correction. If the cover-glass is too thick, decrease the tube length, and if too thin, increase it by means of the draw-tube until the best definition is obtained. For instance, when a certain $4 \mathrm{~mm} .0 .95$ N.A. objective, corrected for cover-glasses $0.18 \mathrm{~mm}$. thick and a $160 \mathrm{~mm}$. tube, was used with thin covers of $0 \cdot 15 \mathrm{~mm}$., it was necessary to lengthen the tube by $30 \mathrm{~mm}$. It is a very difficult matter to make this adjustment, but it may be done by examining a fine line or point in the object, and focussing above and below it; if the image goes out of focus in the same manner both ways the adjustment is correct.

Oil-immersion lenses are not fitted with correction collars, as the cedar oil and cover-slip are practically one optical system, and, within wide limits, the thickness of cover does not influence the performance of the lens.

Tube Length.-All objectives are computed to perform best with a definite tube length, that is distance between the shoulder of the objective and the top of the tube on which the eyepiece rests, indicated in Fig. 22 as the "Mechanical tube length." Unfortunately, all makers do not select the same tube length for which to correct their objectives, but most lenses are now marked with the standard adopted. The old English tube was $10^{\prime \prime}$, but this is seldom used now; most makers have adopted a length 
of $160 \mathrm{~mm}$., but others use $170 \mathrm{~mm}$. or $200 \mathrm{~mm}$. The corrections of high-power objectives are so delicate that care must always be taken to adjust the tube length to

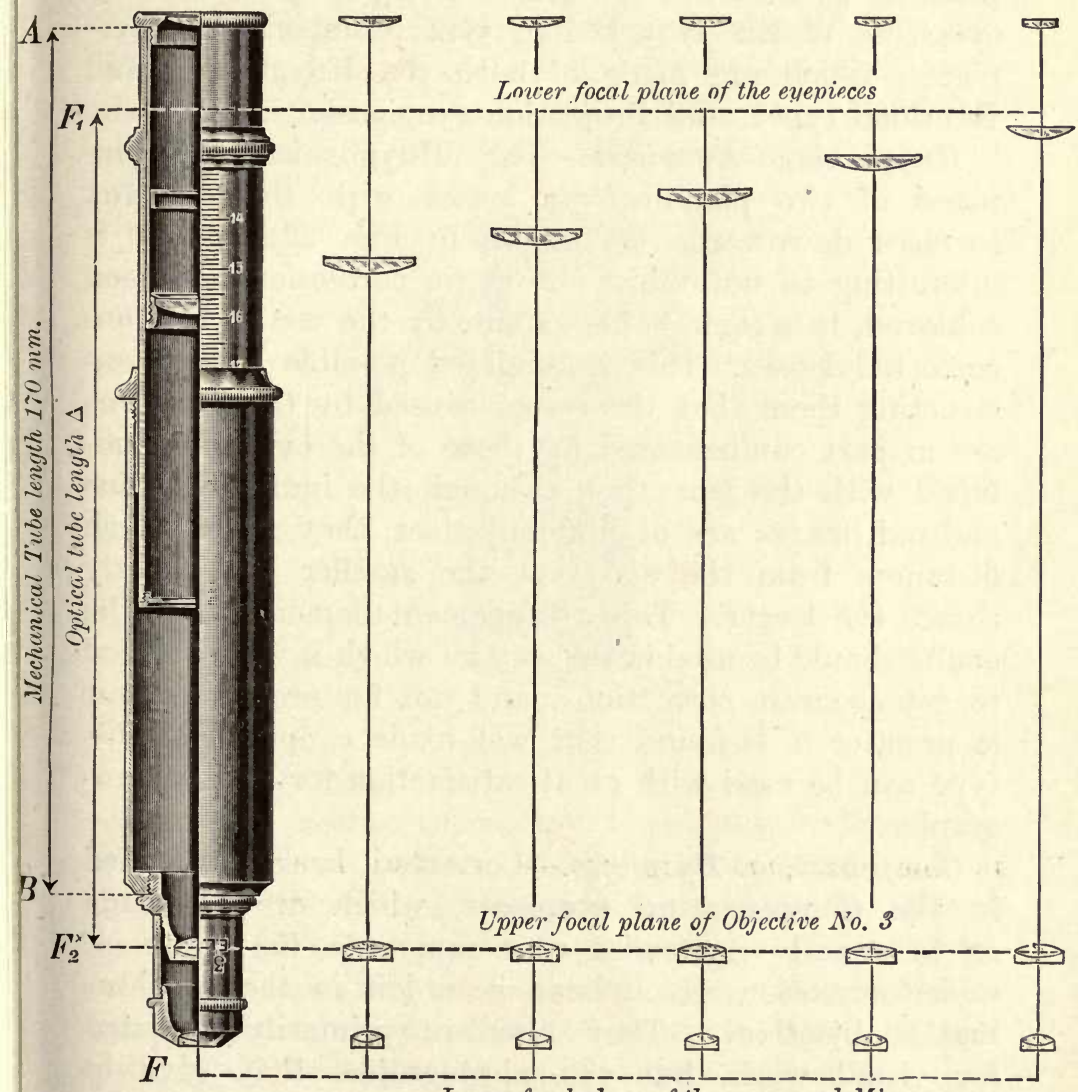

Lower focal plane of the compound Microscope

Fig. 22

MECHANICAL AND OPTICAL TUBE LENGTHS

the objective in use, and to correct for any variation from the standard thickness of cover-glass if the objective has no correction collar. The thickness of any nose-piece or 
other apparatus used between the tube and objective must be taken into account with the tube length.

Oculars or Eyepieces. - There are three kinds of eyepiece in general use for photomicrography. Ordinary eyepieces of the Huyghenian type, Compensating eyepieces, which are made of both the Huyghenian and Ramsden types, and Projection eyepieces.

Huyghenian Eyepieces.-The Huyghenian is composed of two plano-convex lenses with their convex surfaces downwards (as shown in Fig. 22), and it is interesting to note that chromatic correction has been achieved, to a remarkable extent, by the use of two uncorrected lenses. This is rendered possible by so constructing them that the errors caused by the field-lens are in part compensated by those of the eye-lens, combined with the fact that although the individual blue and red images are of different sizes, they fall at such distances from the eye that the smaller one exactly covers the larger. This arrangement demands that the ocular should be used in the way for which it was designed to get accurate corrections, and not for projection, but in practice it is found that well-made eyepieces of this type can be used with great satisfaction for photomicrography.

Compensating Eyepieces.-Corrected lenses are used in the Compensating eyepieces, which are so made as to exactly neutralise or compensate the errors of under-correction which have been left in the apochromatic objectives. They also are primarily intended for visual work, but the photographs they give in conjunction with apochromatic or achromatic objectives of high power leave practically nothing to be desired.

Projection Eyepieces.-Projection eyepieces are specially made for photomicrography to give an image on a screen situated beyond them. They are intended for 
use with apochromatic and high-power achromatic or fluorite lenses, and are only made to give small magnifications, 2 and 4 times for the $160 \mathrm{~mm}$. tube, which become 3 and 6 times if used on the $250 \mathrm{~mm}$. tube. The 4 is the more useful eyepiece of the two. When in use an image of the diaphragm they carry must be focussed on the ground glass by rotating the movable eye-lens (Fig. 23). The graduations serve to

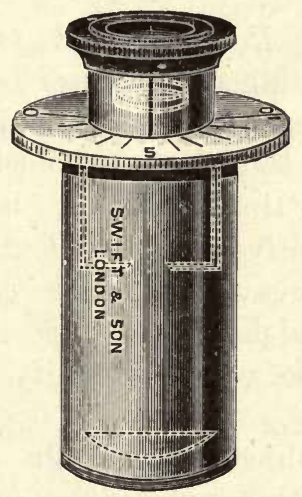

Fig. 23

PROJECTION EYEPIECE

record the position necessary to obtain accurate correction for any camera extension. In practice it is somewhat difficult to get the best focus of the diaphragm, and Messrs. Zeiss supply a tape by which the extension of the camera can be measured, and which indicates by a number corresponding to the graduations the best position of the adjusting ring for that extension.

Demonstration Eyepieces are made by Leitz with a second eye-lens, through which part of the light is deflected by prisms, in order that two observers may examine a preparation at the same time, and they are very useful for instantaneous or other photomicrography in which it is necessary to watch and focus the pre- 
paration right up to the moment of exposure. The method of using them is given on page 247 .

Achromatic and Holoscopic Eyepieces.-There are other types of ocular made, but not so frequently used. For instance, there are achromatic oculars, and the Holoscopic eyepieces made by Watson. The latter are so arranged that by adjusting the separation of their component lenses they may be used with either achromatic or apochromatic objectives at will.

Combination of Eyepiece and Objective.-The best results with low- and medium-power achromatic objectives are usually obtained without an eyepiece, and in any case it is not advisable to use an ocular with greater magnifying power than 8 times. Firstly, because any residual error of the objective is magnified by the eyepiece; and, secondly, because there is considerable loss of light with the higher eyepieces. The most useful eyepieces are those which magnify about 4,6 , and 8 times.

Apochromatic objectives, on the other hand, should always be used with compensating or projection oculars, their corrections are so good and the brilliance of the image so great that very high-power oculars, giving magnifications up to 18 or 27 diameters, can be used without any serious depreciation of the image. The $\times 6$ and $\times 12$ are the most useful of the compensating eyepieces. The higher power achromats ( $\frac{1}{6}{ }^{\prime \prime}$ and over), with large N.A., are also generally under-corrected, and perform to better advantage with a compensating or projection eyepiece than with a Huyghenian.

Most makers now compute their eyepieces so that the lower focal planes fall at the same position in the microscope tube (Fig. 22), and, in consequence, a change of eyepiece may be made with, at most, a slight alteration in the fine adjustment.

Designation of Eyepieces.-There is no uniformity 
among makers in the designation of their eyepieces, some marking them with numbers, others with letters or with their equivalent foci, and others, again, with a magnifying power. Reference must accordingly be made to their catalogues for information as to the power of the respective eyepieces. Those marked A, B, C, etc., and 1, 2, 3, etc., are always in ascending order of magnification, but the same intervals between each are not used by all makers who ađopt one or the other notation. Even when magnifying power is marked, the information is not of great use unless the tube length for which the maker in question adjusts objectives, and the convention he adopts for expressing their initial power, are known.

Magnification.- The magnification produced in a photomicrograph is determined most accurately by replacing the object, after exposure has been made, by a stage micrometer, or slide on which lines $\frac{1}{100}$ and $\frac{1}{1000}$ of an inch, or $\frac{1}{10}$ and $\frac{1}{100}$ of a $\mathrm{mm}$. apart are ruled, and measuring the intervals between the lines when focussed on the ground glass. This should always be done, when exact measurements are required, before any alteration is made in the camera extension or other parts of the apparatus. Sliding calipers with verniers reading to $\frac{1}{10} \mathrm{~mm}$., or $\frac{1}{100} 0^{\prime \prime}$, are very useful for measuring the distance between the lines on the ground glass, and the mean of several divisions should be taken to avoid slight errors in graduations on the micrometer. The magnification produced is the quotient of the measured distance between any number of lines and their actual separation on the micrometer. Thus, if three of the $\frac{1}{100}{ }^{\prime \prime}$ graduations cover $4 \cdot 2^{\prime \prime}$ on the ground glass, the magnification is $4 \cdot 2$ divided by $\frac{3}{100}$, or 140 diameters.

The magnification given by a lens is measured by the distance between its upper focal plane and the image plane, divided by the equivalent focal length. 
The magnification produced by a microscope depends on two factors:-

1. The magnification produced by the objective in an image formed in the lower focal plane of the eyepiece, called the "initial magnifying power" of the objective, and expressed by-

$$
\frac{\Delta}{\text { focal length of objective }}
$$

where $\Delta$ is the optical tube length, that is the distance between the upper focal plane of the objective and the lower focal plane of the eyepiece (see Fig. 22).

2. The magnification of that image by the eyepiece when forming the final image, supposed to exist at the normal minimum distance of distinct vision from the eyepoint, called the power of the eyepiece, and expressed by-

250

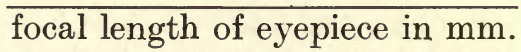

or

10

focal length of eyepiece in inches

There are two other factors which govern magnification in photomicrography, besides the powers of the objective and eyepiece, and they are :-

3. The tube length, on which will depend the initial power of the objective as defined above.

4. The camera extension, or distance between the upper focal plane of the eyepiece and the ground glass.

In practice the positions of the upper and lower focal planes of the objective and eyepiece (shown in Fig. 22 for definite lenses) are generally unknown to the worker, and consequently it is convenient to make all measurements of magnifications at some standard distance from given points on the microscope. A table 
should be drawn up, showing the magnification produced by every possible combination of lenses at this standard image distance, the tube length given by the maker being always maintained. When using the objective alone the image should be formed $10^{\prime \prime}$, or $250 \mathrm{~mm}$., from the shoulder of the objective, and when an eyepiece is used the camera extension should be $10^{\prime \prime}$, or 250 $\mathrm{mm}$., measured from the top of the tube on which the eyepiece rests. The magnifications given in the table of objectives and eyepieces, page 8, approximate to those found under these conditions. Such a table is very useful, as the magnification produced in any photomicrograph can be instantly determined, with quite sufficient accuracy for most purposes, without the trouble of actual measurement, if the baseboard of the camera is furnished with a scale to measure the image distance.

Camera Extension and Magnification.-The effect produced by any variation in camera extension, measured from the objective or eyepiece according to circumstances, and the result of alterations in the tube length from the standard adopted by the maker of the objective are determined as follows without any great error. The factors obtained being used to multiply the magnifications given in the table :-

Factor for camera extension-

extension in inches 10

Factor for tube length- extension in $\mathrm{mm}$.

$$
250
$$

actual tube length

standard tube length

Magnification and Tube Length.-The camera extension may be varied as desired to influence the magnification, but the tube length must only be altered with caution. With high powers any alteration from the standard, unless 
made to counteract cover-glass thickness, will introduce spherical errors. With low powers the error introduced in this way is insignificant, and the tube length may be altered to obtain a final adjustment of magnification to plate size, etc., but the method should only be resorted to when no other is available. This factor is consequently not often used, unless the tube length is altered to correct for cover-glass thickness.

Initial Power of the Objective and Power of the Eyepiece.-When making up a battery of lenses it is necessary to know their powers. The magnification an objective will produce in a photomicrograph at $10^{\prime \prime}$ image distance can always be roughly determined by dividing the image distance by its focal length $\frac{10}{\mathrm{f} \text {. in inches }}$ or $\frac{250}{\mathrm{f} \text {. in } \mathrm{mm} \text {. }}$ And most makers follow Abbe's suggestion, and call this its "initial magnification." But at least one, Leitz, uses the term "initial magnification" in the sense defined by $\frac{\Delta}{\text { focal length }}$.

Whichever method is adopted, the table of magnifications for objectives and eyepieces given in the maker's catalogue will give the magnifications produced in an image at $10^{\prime \prime}$ from the eyepiece; but in order that this may be so, the power of the eyepieces must be expressed in accordance by-

$$
\frac{\Delta}{\text { focal length of eyepiece, }}
$$
or if $\Delta$ occurs in objective factor by-

$$
250
$$

focal length of eyepiece.

Consequently the values given in the catalogues for the powers of the eyepieces are of no use unless used with that maker's objectives, or with those of another maker who expresses the initial magnifying 
power under the same convention. This confusion is made all the worse by the various tube lengths, 160 , 170,200 , and $250 \mathrm{~mm}$., employed by different makers, and unless all the factors are known the power engraved on the eyepiece, or the value given in the catalogue for the letter or figure used in its place, cannot be used as the basis of an estimation of the magnification produced when using objectives and eyepieces by different makers. Two eyepieces of the same focal length may be called by different makers $\times 10$ and $\times 7$, and therefore the practical worker is driven to make up his own table of magnifications, unless he uses one make of lenses only.

Small Photographic Objectives.-Many makers construct small lenses on the lines of photographic anastigmats of $1^{\prime \prime}, 2^{\prime \prime}$, and $3^{\prime \prime}$ focal length $(25,50$, and $75 \mathrm{~mm}$.), fitted with the R.M.S. screw for microscopes. To these they give special names, such as Planar, Micro-Summar, Holostigmat, etc. They are largely used for low-power photomicrography, and have an advantage in flatness of field and covering power over ordinary microscopic objectives. They are used without eyepieces, and should be chosen for general views of large objects, for which their magnification is sufficient. Their aperture is given in the "f" ratio notation commonly used for photographic lenses, denoting the ratio of the aperture to their focal length, and forming a convenient measure of their rapidity.

Comparison of " $f$ " Ratio and N.A.-

$$
\mathrm{N} \cdot \mathrm{A} .=\frac{\mathrm{m}}{2 \mathrm{f} \times(\mathrm{m}+\mathrm{l})}
$$

is a convenient formula for converting " $f$ " ratios into terms of N.A.; in it $\mathrm{m}=$ magnification.

If, as a concrete case, the " $f$ " aperture of one of 
these lenses is taken, and the N.A. calculated, the latter will be found to be small. A lens with an aperture given by $f / 4 \cdot 5$, and used to obtain a magnification of 4 diameters,

is working with a N.A. given by N.A. $=\frac{4}{9 \times(4+1)}=\cdot 09$. This will indicate that no high degree of resolution is to be expected, but for the purposes for which these lenses are intended this is of small account, their flatness of field is a much more useful property.

These lenses are fitted with an iris diaphragm, which is used to close down the aperture when greater depth of focus is desired. If the iris is not placed in quite the correct position in the lens, it will restrict the field of view as it is closed down. They will, in some cases, cover an object whose diameter is as much as their focal length.

The magnification they give under various conditions of camera extension can be calculated from the formula

$$
\mathrm{M}=\frac{\mathrm{E}}{\mathrm{F}}-1
$$

Where $\mathrm{M}=$ magnification.

$\mathrm{E}=$ distance of focussing screen from diaphragm of lens.

$\mathrm{F}=$ focal length.

It is interesting to note that some of these objectives can be used on small pocket cameras, so giving them a double usefulness. If the lens is unsymmetrical, it should be placed in the camera with the lens which faces the object in photomicrography turned towards the focussing screen.

Sets of Objectives and Eyepieces.-A Complete Set of Objectives comprises the following lenses given in tabular form with approximate magnifications measured under the conditions described above. 



\section{Proboscis of Blow-fly.}

These two photomicrographs, together with those reproduced on Plate 9, are given to illustrate the wide range of magnification obtainable with a very small battery of lenses.

Objectives of $1^{\prime \prime}$ and $\frac{1}{4}^{\prime \prime}$ focal length and No. 3 eye-piece only were used, with extensions varying from $5^{\prime \prime}$ to $20^{\prime \prime}$.

\begin{tabular}{|c|c|c|c|}
\hline & & A & B \\
\hline Magnification . & $\ldots$ & 15. & 35. \\
\hline Objective .... . & $\ldots$ & \multicolumn{2}{|c|}{ Swift's 1" Achromat. } \\
\hline Eye-piece .... & $\ldots$ & None. & Swift's No. 3. \\
\hline Extension & $\ldots$ & $15^{\prime \prime}$ (from objective). & $5^{\prime \prime}$ (from eye-piece). \\
\hline Condenser & $\ldots$ & $\begin{array}{r}\text { Watson's Universal } \\
\text { front lens }\end{array}$ & $\begin{array}{l}\text { Achromatic, with } \\
\text { removed. }\end{array}$ \\
\hline Illuminant & $\ldots$ & Incandesc & ent gas. \\
\hline Light Filter & $\ldots$ & Saturated solution & of copper acetate. \\
\hline Plate & $\ldots$ & Imperial Special & Rapid Ortho. \\
\hline
\end{tabular}




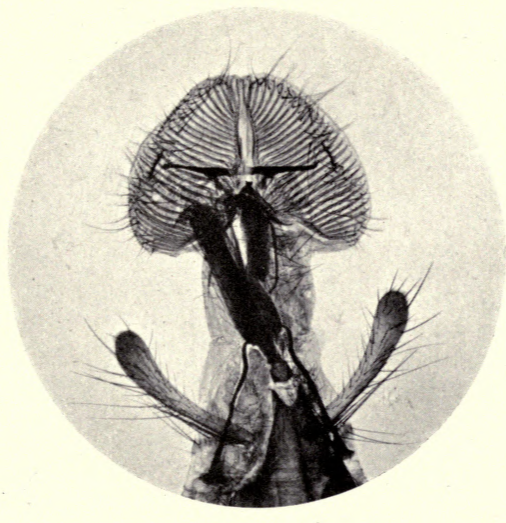

A

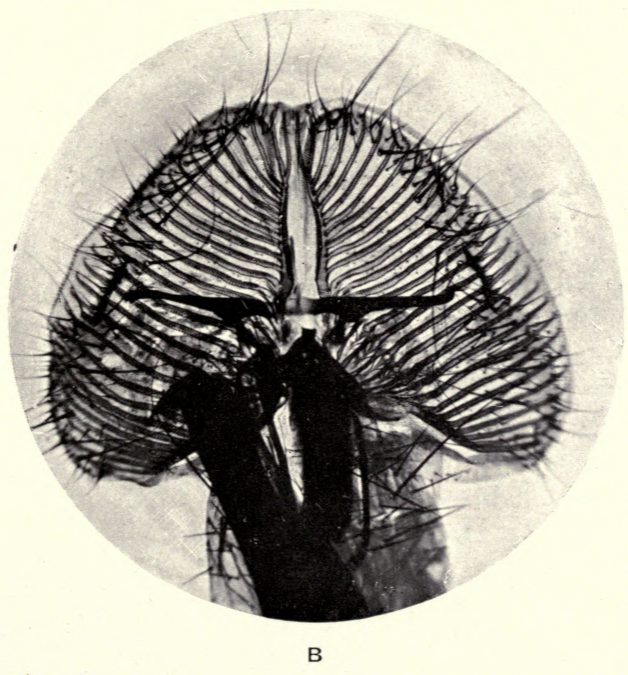






\section{Proboscis of Blow-fly.}

(See also Plate 8.)

\begin{tabular}{|c|c|c|}
\hline & & B \\
\hline Magnification & $\ldots$ & 105. \\
\hline Objective ... & $\ldots$ & Swift's $1^{\prime \prime}$ Achromat. \\
\hline Eye-piece ... & $\ldots$ & Swift's No. $3 \times 7$. \\
\hline Extension & $\ldots$ & $10^{\prime \prime}$. \\
\hline Condenser & $\ldots$ & $\begin{array}{l}\text { Watson's Universal, Universal. N.A. 1.0. } \\
\text { front lens removed. }\end{array}$ \\
\hline Illuminant & $\ldots$ & Incandescent gas. \\
\hline Light Filter & $\ldots$ & Saturated solution of copper acetate. \\
\hline Plate & $\ldots$ & Imperial Special Rapid Ortho. \\
\hline
\end{tabular}

The proboscis of Blow-fly is a very favourite test object for lowpower objectives. It must be mounted quite flat, and examined with a $\frac{3}{4}$ or $\frac{7}{8}$ cone of critical light. Under these conditions the spirals on the suctorial tubes should be clearly defined, and the fine hairs round its margin shown distinctly and without blurring. 


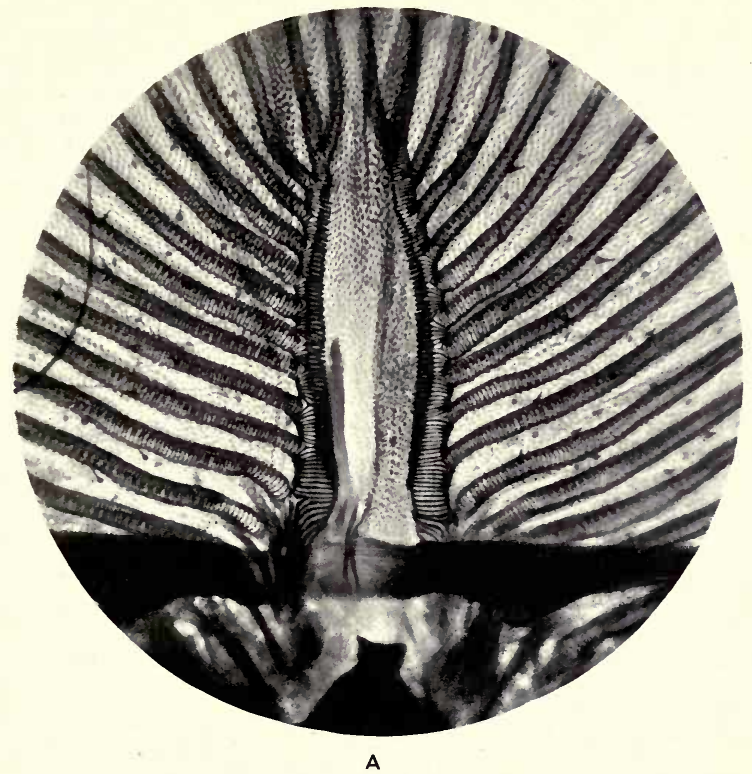

A

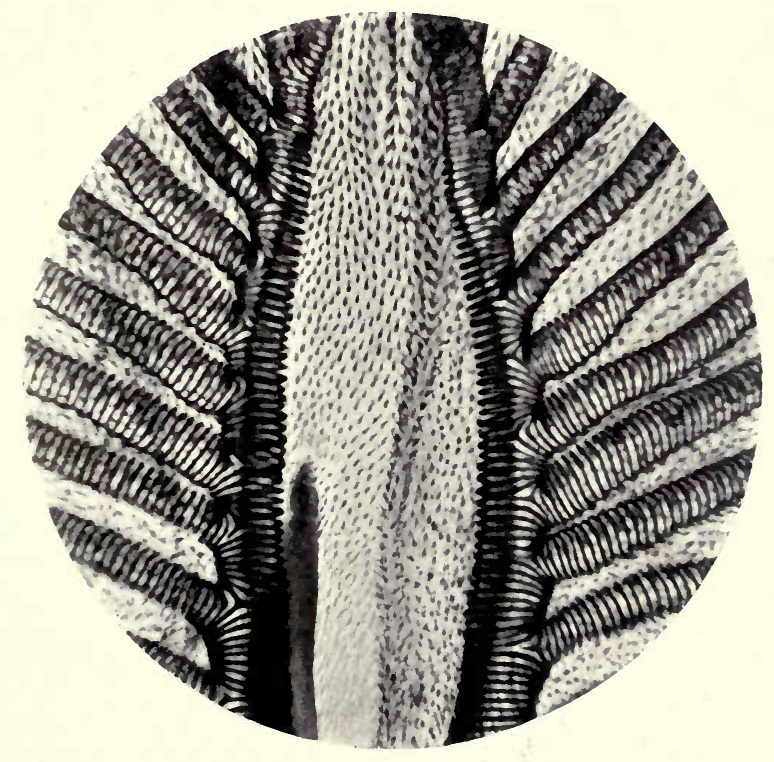





\begin{tabular}{|c|c|c|c|c|c|c|c|}
\hline \multicolumn{2}{|c|}{ Focal length. } & \multirow{3}{*}{$\frac{\text { Suitable. }}{\text { N.A. }}$} & \multicolumn{5}{|c|}{$\begin{array}{l}\text { Magnifications at } 10^{\prime \prime} \text { from Oljective or } \\
\text { Eyepiece. }\end{array}$} \\
\hline \multirow{2}{*}{$\mathrm{mm}$. } & \multirow{2}{*}{ inch. } & & \multirow{2}{*}{$\begin{array}{l}\text { Objective } \\
\text { used } \\
\text { alone. }\end{array}$} & \multicolumn{4}{|c|}{ With Ocular. } \\
\hline & & & & $\times 4$ & $\times 6$ & $\times 8$ & $\times 12$ \\
\hline 75 & 3 & $\cdot 10$ & 3 & 12 & 18 & 24 & 36 \\
\hline 48 & 2 & $\cdot 15$ & 5 & 20 & 30 & 40 & 60 \\
\hline 36 & $1 \frac{1}{2}$ & $\cdot 17$ & 6 & 24 & 36 & 48 & 72 \\
\hline 24 & 1 & $\cdot 2-\cdot 3$ & 10 & 40 & 60 & 80 & 120 \\
\hline 18 & $\frac{3}{4}$ & $\cdot 2-.35$ & 13 & 52 & 78 & 104 & 156 \\
\hline 16 & $\frac{2}{3}$ & $\cdot 25-\cdot 4$ & 15 & 60 & 90 & 120 & 180 \\
\hline 12 & $\frac{1}{2}$ & $\cdot 35-\cdot 5$ & 20 & 80 & 120 & 160 & 240 \\
\hline 8 & $\frac{1}{3}$ & $.5-.65$ & 30 & 120 & 180 & $240^{\circ}$ & 360 \\
\hline 6 & $\frac{1}{4}$ & $.65-.9$ & 40 & 160 & 240 & 320 & 480 \\
\hline 4 & $\frac{1}{6}$ & $.7-.95$ & 60 & 240 & 360 & 480 & 720 \\
\hline 3 & $\frac{1}{8}$ & $.8-.95$ & 80. & 320 & 480 & 640 & 960 \\
\hline $2 \cdot 5$ & $\frac{1}{10}$ & $1 \cdot 3$ & 100 & 400 & 600 & 800 & 1200 \\
\hline 2 & $\frac{1}{12}$ & $1 \cdot 2$ to $1 \cdot 4$ & 120 & 480 & 720 & 960 & 1440 \\
\hline
\end{tabular}

And Small Photographic Lenses of 3", 2", and 1" or 75,50 , and $25 \mathrm{~mm}$. focal length.

A battery sufficient for practically any purpose should include 3 " photographic lens.

$$
1 \frac{1}{2} \text { ", } \frac{2}{3}, \frac{1}{3}{ }^{\prime \prime}, \frac{1}{6}, \frac{1}{12} 2^{\prime \prime} \text { objectives, }
$$

with the following oculars :-

$\times 12$ Compensating.

$\times 4$ Projection.

$\times 6$ and $\times 8$ Huyghenian or Compensating.

Convenient powers for less ambitious sets, but ample for most workers, are-

(i) $3^{\prime \prime}$ planar, $1 \frac{1}{2}, \frac{2}{3}, \frac{1}{6}$ ", and $\frac{1}{12}$ " oil-immersion, with $\times 4$ and $\times 8$ eyepieces. 
(ii) $2^{\prime \prime}, \frac{1}{2}{ }^{\prime \prime}, \frac{1}{8}$, , or $\frac{1}{10}{ }^{\prime \prime}$ oil-immersion, with $\times 4$ and $\times 8$ eyepieces.

(iii) In cases where the higher powers are most frequently used, $\frac{2}{3}^{\prime \prime}, \frac{1}{6}$, , and $\frac{1}{12}$ " oil-immersion, with $\times 4$ and $\times 8$ eyepieces.

(iv) If only two are bought, a great deal can be done with $1^{\prime \prime}$ and $\frac{1 "}{6}$ with $\times 4$ and $\times 8$ eyepieces. 


\section{CHAPTER IV}

\section{LAMPS AND ILLUMINANTS}

Critical Light.-This subject is discussed in Chapter VIII, but it is necessary before describing the different illuminants available for photomicrography to state that the term "Critical" is used in reference to the most delicate microscopical work necessitating the employment of special methods of illumination- "Critical Illumination," and that the conditions for the best performance of the objective, and the formation of the "Critical Image," are attained when an image of the radiant is projected across the field of view by means of a substage condenser whose back lens is filled with light; the image of the radiant being focussed simultaneously with that of the object.

Suitability of various Radiants for Photomicrography.The great difficulty connected with illumination in photomicrography is to get critical light, and at the same time fill the field of the objective evenly and with sufficient intensity. Several methods, depending on the magnification of the image of the radiant until it covers the field of view, are described in Chapter VIII, and they demand that the intensity of light should be uniform all over the radiant surface. Many of the difficulties encountered in lens making would be surmounted and the errors of lenses more easily corrected if a point source of light could be utilised. This is, however, unattainable in practice, and the nearest approach to it must be sought; a circular radiant 
surface of about $\frac{1}{8}$ inch in diameter would be very suitable.

No lamp gives a perfect illumination and the drawbacks of a few of the most convenient must be considered, it will then be easier to select the most suitable in any given circumstances. The intensity of large radiants is not even over the surface, and nearly all show structure or inequalities of light when their image is focussed across the object. The mesh of an incandescent mantle, and inequalities in a flame, or in the crater of an arc lamp, are cases in point.

The light must be absolutely steady, and, for convenience, it should always give the same illuminating effect. The less heat given off by a radiant the better; the more intense illuminants suffer greatly in this respect, and it is dangerous to use them with delicate preparations or valuable objectives without a cooling trough.

The spectrum of the radiant is a point of importance, constant results cannot be obtained unless the composition of the light is invariable. Monochromatic light is very beneficial in certain directions; the results of defects existing in the objective can be minimised, and the photomicrograph improved by its employment (Chapter IX). The ease with which it can be obtained from any given light source must therefore not be overlooked.

SUNLIGHT.-The sun would be the best of allilluminants if its light could be relied on for use at any time, but even in the most favourable localities some form of Heliostat is necessary to project a steady beam into the microscope. Heliostats are complicated and expensive instruments, and, considered in conjunction with the uncertainty of sunlight, generally put the sun out of court as a source of illumination for photomicrography. The variation of the actinic rays of daylight at different times of the day also introduces uncertainty in the exposure of the 
photographic plate, and, on that account, the relative constancy of an artificial illuminant is a great point in favour of always using one or other form in preference to daylight. It is possible to dispense with a clockwork heliostat and direct the beam of sunlight by a careful manipulation of the mirror of the microscope, or, better still, to set up a second reflector in such a position that it can be made to follow the sun, and throw the light on to the substage mirror. This simple device is scarcely satisfactory, however, as it is not possible to control the illumination very exactly thereby.

North light, or sunlight reflected from white clouds, is not suitable for critical work, and is not of sufficient intensity for high powers, but is very convenient for low-power work. This is particularly the case when a vertical camera, which can be readily moved into a good position, is used.

ELECTRIC LIGHT.-On account of the difficulties involved in the use of the sun as an illuminant for photomicrography recourse must be had to artificial radiants, and of these the electric arc stands pre-eminent. Under the best conditions a perfectly steady light is obtained, and, with direct current, the crater forms a radiant surface that closely approximates to a small even disc.

Arc Lamps.-These are made for direct and alternating supplies, but a perfectly steady light is never obtained from an alternating current, and the are generally moves so markedly as to be useless for critical work. For many purposes, however, when a brilliant but not critical light is required, an alternating are can be used, but very great care is needed in the adjustment to prevent uneven illumination, which may occur in the few moments intervening between final adjustment and exposure, or even in the middle of the exposure.

Arc lamps are made to work with almost any current from 4 to 60 ampères or upward, giving a light that 
varies correspondingly from 300 or 400 to several thousand candle-power. The most useful for the purpose under consideration are the smaller types taking 5 ampères or less; occasionally the larger lamps may be employed with advantage, for cinematographic exposures for example, but as a rule no greater illumination than 500 candle-power is required. The small lamps have the very great advantage that they can be run with safety on ordinary wiring fitted with 6-ampère fuses. Most supply-circuits are run at voltages of 110 to. 250 , and as arc lamps only require 20 to 65 volts, according to their power, a resistance must be connected to them to reduce the pressure to that extent. Suitable Resistances or Choking Coils are supplied by the lampmaker according to the voltage of the supply and its periodicity if alternating; they, of course, involve a considerable waste of current by conversion into heat.

When obliged to work on an alternating supply a rectifier should be installed; of these the Cooper-Hewitt mercury rectifier is very suitable, and is supplied for any voltage and periodicity; a Nodon rectifying valve or a motor transformer can also be employed. Any of these devices involves considerable outlay and it is frequently advisable on that account to turn to some other source of illumination for purposes for which the alternating arc is unsatisfactory.

The lamp must be so constructed that the crater which forms on the positive carbon, and is the source of light, faces the microscope with as little obstruction as possible from the negative carbon, and in such a way as to avoid loss of light backwards. In some lamps the carbons are at right angles, the positive being horizontal and the negative vertical; in others there is a smaller angle between them, while in others they are almost in line, but inclined at about $30^{\circ}$ to the vertical with the lower negative carbon slightly in front of the upper 
positive, so that the crater is drawn forward towards the microscope.

There must be some arrangement to maintain the correct gap between the two carbons; it may be automatic or depend on adjustment by hand. The automatic is undoubtedly the better, provided the mechanism works smoothly; and a lamp so fitted should give a constant light, and require no further attention when once set in the optical axis of the microscope.

Automatic Arc Lamps. - A description and figure of

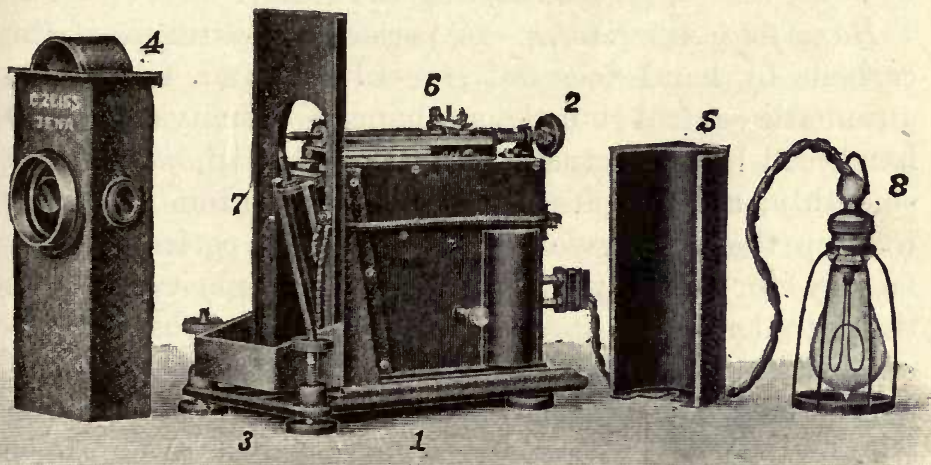

Fig. 24

ZEISS' 5-AMPÈLE AUTOMATIC AliC LAMP

Zeiss' 5-ampère automatic arc lamp is given as an example of the type of lamp best adapted for photomicrography. Siemens' Al Carbons are used, inclined to each other at an angle of about $60^{\circ}$. The horizontal positive carbon is cored, and has a diameter of $5 \mathrm{~mm}$., the negative is solid and of $4 \mathrm{~mm}$. diameter. After careful adjustment by the milled head (2) the carbons are regulated by clockwork mechanism wound up by the screw (1). This causes a slight forward movement of the carbons, every twenty seconds, and the 
desired gap between them is maintained with great accuracy.

The whole of the lamp is enclosed, the front (4) carrying a small aplanatic condenser with a N.A. of $0 \cdot 4$, and an iris diaphragm. There is also a dark glass window through which the arc can be examined.

A lamp of similar appearance is made for hand regulation, but the carbons are arranged at right angles to each other. Either lamp can be carried on a saddle.stand for the optical bench, but that illustrated is fitted with levelling screws (3) for centring the crater.

Hand-feed Arc Lamps.-A periodic adjustment of the carbons by hand does not give so regular a feed as an automatic control ; but the mechanism on many of the new hand-feed lamps, actuated by a milled head, works very smoothly, and despite the constant attention necessary to keep the arc regular, these lamps are perfectly satisfactory for most purposes. Their simplicity enables them to be made at a much lower price than a corresponding automatic lamp, and they are at present undoubtedly one of the most popular illuminants obtainable for photomicrography. They usually run on about 4 ampères current, and so can be used with safety on any ordinary wiring. With suitable adjustments and resistances they can be used either on direct or alternating supply, and give from 300 to 500 candle-power.

r. The "Liliput" lamp of Leitz is shown at Fig. 1, while Baker has more recently introduced a somewhat similar lamp. Both of them carry a bull's-eye condenser, focussing in a sliding mount and a hood which surrounds the light. In Baker's an image of the carbon is projected through a pin-hole on to a ground-glass disc for examination; the Liliput is fitted with a dark blue glass window. Cored carbons are used, arranged at right angles to each other. A single milled head actuates the feed in both lamps ; in Baker's model it is so geared that the horizontal 
positive carbon moves forward at a greater rate than the vertical negative, the speeds being adjusted to accurately counterbalance the rates of burning. In the Liliput a considerably narrower negative carbon is used, $6 \mathrm{~mm}$. in diameter, as against an $8 \mathrm{~mm}$. positive. These burn away at the same speed and are correspondingly moved forward by the adjustment.

Small lamps of this kind are connected directly to a wall-plug or lamp-holder, with a suitable resistance placed in the circuit, and it is important that the wires be connected to the proper lamp-terminals. Fig. 25 shows the method of connection. A flexible lead is fitted to a lamp-holder and its positive and negative wires determined by means of pole paper-paper coated with potassium iodide and starch, for instance. The paper is moistened and the ends of the two wires placed on it near together; the positive wire gives a blue patch. This wire is then connected to one terminal of the resistance, and the other terminal joined to the positive pole of the lamp by means of a length of wire. The circuit is completed by joining the other wire of the supply cable direct to the negative lamp-terminal.

The lamps are adjustable for height on a suitable pillar, and can be tilted if required. The carbons have to be carefully adjusted until their points just touch when moved forward, and so that the negative carbon does not obstruct the crater which forms on the horizontal positive carbon. The are is struck by momentarily bringing the two carbons into contact, by means of the milled head, and then withdrawing them slightly; a good crater forms in a minute or so, and then a steady light is given.

Enclosed Arcs-Alternating Current.-Enclosed arc lamps are very popular for projection purposes, and can be used for photomicrography. They have the disadvantages, in the forms at present made, that disturbing reflections are given by the enclosing glass cylinder, and 
that the carbons are liable to give off a flame which prevents a clear image of the crater being obtained.

With alternating current, on the other hand, they have a distinct advantage over the open arcs already described, in that the arc is steadier and less given to searching; they are, however, still not suitable for critical illumination. The greater length of time for which they will burn without adjustment, when com-

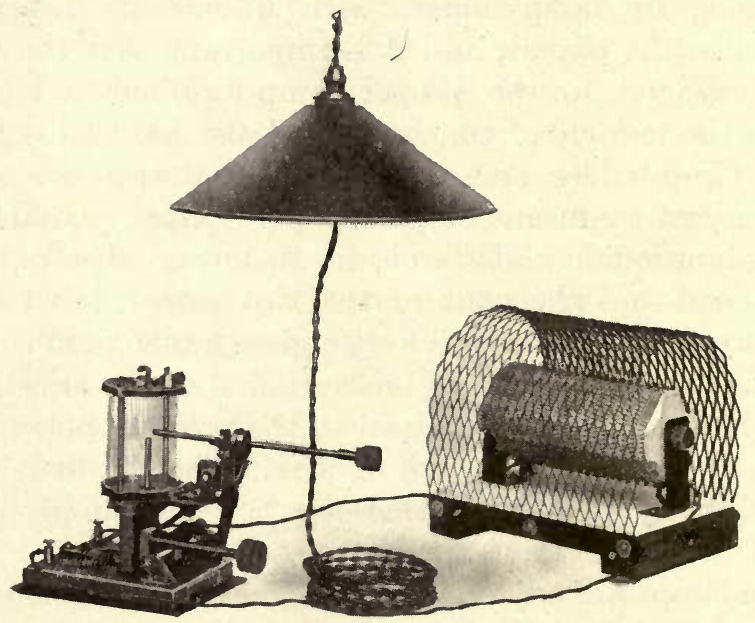

Fig. 25

ENCLOSED ARC LAMP

pared with an open arc, is not of such great importance in photomicrography as for projection.

Fig. 25 shows a very handy form made by the Westminster Engineering Co., Ltd.

If the Liliput lamp is used on an alternating circuit, two carbons of equal diameter are used; and in order that the arc may be as steady as possible, they should be the smaller ones of $6 \mathrm{~mm}$. diameter. The supply wires are connected to the lamp-terminals indiscriminately. 
Incandescent Filament Lamps. - Ordinary filament lamps are not at all suitable for photomicrography, as the filament is so thin that it is practically impossible to obtain a sufficiently broad image of it to fill the field of the objective. They may sometimes be used, in ground-

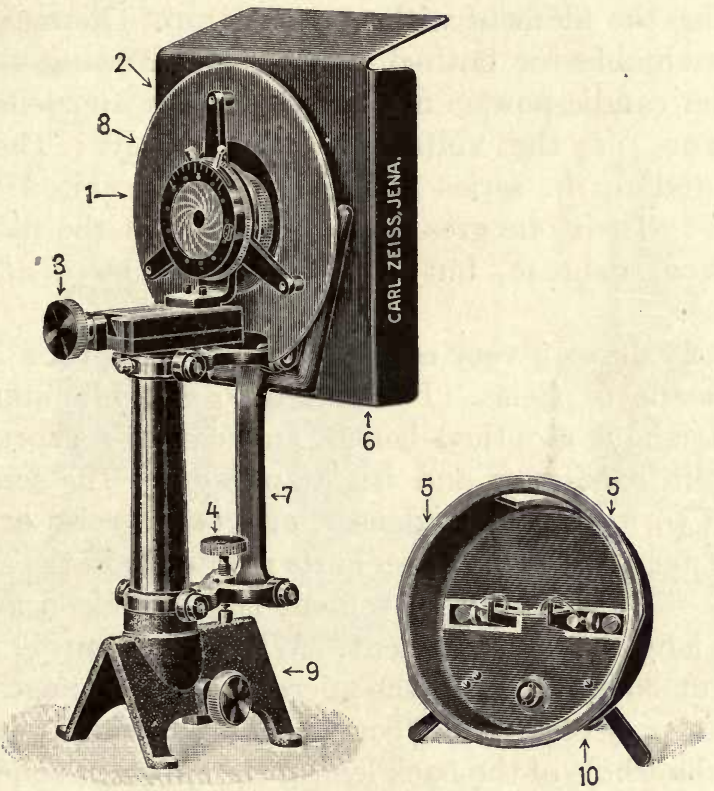

Fig. 26

ZEISS' NERNST LAMP

glass globes, for low-power work, but they are never very satisfactory, as their intensity is low.

Nernst Lamps. - The Nernst lamp is a very convenient illuminant in certain of its forms. The usual l-ampère type has a single straight filament broad enough to fill the field of an objective with even illumination when a suitable condenser is used, and of sufficient intensity to use with high powers. There are other types with more 
than one filament, or with heaters coiled round a single filament, but these cannot be used for critical work, as the spirals of the heater give the appearance of dark bands across the light when focussed on to the object plane. There is no need to use a lamp fitted with an automatic heater, as it is quite easy to start the light merely by warming the filament with a spirit-lamp. Nernst lamps are obtainable for both direct and alternating current, and the candle-power may be taken as approximately the same as the voltage of the supply. They are connected up in series with a resistance suited to the voltage. Their one great drawback is that the filaments are rather delicate, but they can be replaced 'at small expense.

Fig. 26 shows a very convenient mounting for a Nernst lamp made by Zeiss. It is fitted to a saddle stand for their triangular optical bench, and carries a short focus aplanatic condenser and iris diaphragm. The screw (3) is used to focus the condenser, and (4) to raise or lower the filament which is shown in its mounting in the smaller figure. No horizontal movement is required on account of the length of the filament. When the lamp is placed at about 30 inches from the microscope, the image of the filament projected by the condenser is sufficiently large to cover the whole of the back lens of the substage condenser. Another convenient mount for a Nernst lamp is shown at Fig. 8.

Mercury Vapour Lamp.-The spectra of all the foregoing radiants consist of a continuous band of colours, similar to the well-known solar spectrum, but varying in the proportions of rays of different colours. Incandescent vapours, on the other hand, give spectra consisting of isolated bands or lines in various positions, depending on the nature of the vapour. Lamps in which an incandescent vapour forms the source of light are very useful when monochromatic light is required, as it is a 
simpler matter to cut off the few superfluous bands and leave light of one wave length, than to find a filter which will pass rays of one wave length only from light having a continuous spectrum. The vapour of mercury has been found most convenient for this purpose, and lamps are made consisting of a highly exhausted glass tube, containing a small quantity of mercury which is vaporised on the passage of the current, forming a conductor between the two poles and glowing with considerable intensity.

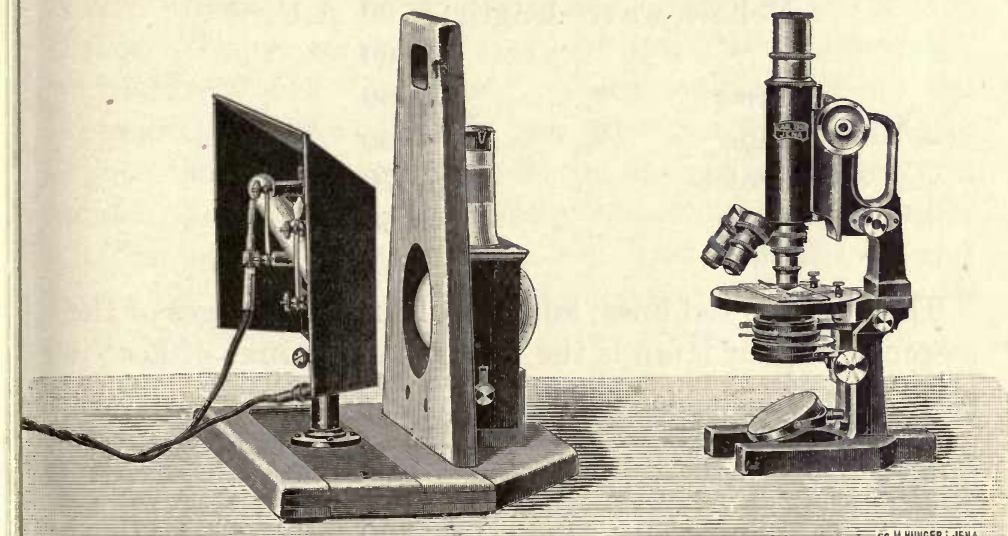

Fig. 27

THE HAGEH MERCURY VAPOUR LAMP

The intensity of the monochromatic light obtained from the mercury vapour lamp by the use of suitable light filters is sufficient for high powers, but unfortunately the tubes are rather fragile and expensive to replace, and its use has so far been rather limited on that account. Tubes made of quartz are sometimes used; they are better in wearing quality than those of glass, but are more expensive.

Fig. 27 is an illustration of the Hageh mercury vapour lamp made by Zeiss. The tube is about $20 \mathrm{~cm}$. long and carried on an adjustable support. Flasks of some 
300 c.c. capacity are supplied, to hold suitable solutions for light filters, and to act as condensers.

To start the lamp the positive and negative supply wires are joined to the proper terminals of the tube, and the latter is slowly tilted to cause the mercury to flow from one end to the other and back again, repeating the movement if the lamp does not begin to glow.

The following are the lines of the mercury vapour spectrum :-

$\begin{array}{cccc}\text { Yellow wave length } & 5790 & \text { A.U. } \\ , & , & 5760 & , \\ \text { Green } & , & 5460 & , \\ \text { Blue } & , & 4360 & , \\ \text { Violet } & , & 4070 \quad, \\ \quad, & , & 4050 \quad,\end{array}$

There are no red lines, and one of the advantages of the mercury vapour lamp is the very small amount of heat it gives.

Suitable solutions to act as light filters can be made as follows :-

1. To transmit yellow light $\lambda=5790$ and 5760 A.U.

Potassium bichromate. . . 15 gram.

Copper sulphate . . . . $3 \cdot 5$,

Sulphuric acid . . . . 1 c.c.

Distilled water . . . . 300 c.c.

2. To transmit green light $\lambda=5460$ A.U.

Picric acid .

0.4 gram.

Copper sulphate . . . . $3 \cdot 5$,

Didymium nitrate . . 15 ,

Distilled water . . . . 300 c.c.

If the Didymium nitrate is omitted from this filter, yellow-green light is passed of wave lengths 5790, 5760, and 5460 . 
3. To transmit blue light $\lambda=4360$ A.U., and also the violet lines 4070 and 4050 A.U.

Copper sulphate .

Distilled water

Ammonia $\cdot 880$
1 gram.

225 c.c.

75 c.c.

LIMELIGHT.-Limelight is so largely used among lanternists that many who take up photomicrography will have it at hand, and will find the ordinary lantern jets quite satisfactory for the purpose, when used with hard limes. There are three types of jet, "Blow-through," "Mixture," and "Injector." The blow-through are the simplest, but the mixture jets give the most powerful light; the latter require both the oxygen and hydrogen under pressure incylinders, and the gases mix in a chamber in the jet. The Gwyer is an excellent mixture jet and gives a brilliant light. For most purposes the blow-through jets are preferable because of their greater safety and simplicity ; hydrogen is seldom used with them, as coal gas from the mains is an efficient substitute, and requires no cylinder. Oxygen is always obtained under pressure in cylinders, which should be fitted with a regulator. The oxygen is connected to the inner jet of the blowthrough burner, and the coal gas to the outer annular tube. The coal gas is first lighted and turned fairly well up to play over the lime, the tap connecting the oxygen cylinder to the regulator is then opened slightly more than will be needed to supply a sufficient current of oxygen. The regulator having thus been put in connection with the cylinder, the tap connecting it to the jet is opened, and then the tap of the jet itself, which last is used to regulate the flame. Efficient regulation cannot be obtained if the oxygen is admitted straight through to the jet without previously connecting the cylinder to the regulator. If coal gas and hydrogen are unobtainable an ether saturator may be used in connection with a blow- 
through jet. The oxygen passes through the saturator and carries ether vapour with it to the jet, and the mixture of oxygen and ether frequently gives a better light than oxygen and coal gas. The distance of the jet from the lime is of importance, as if it is too far away the flame is too large, if too close there is not sufficient light. Experience will soon indicate the best position to get a compact light of great intensity - the jet is usually between $\frac{1}{4}$ and $\frac{3}{8}$ inch from the lime. The candle-power generally attained varies from 500 to 1000 , and with mixture jets 1500 and more can be obtained.

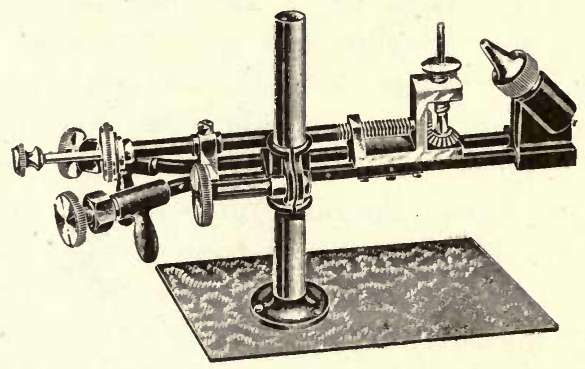

Fig. 28

INJECTOR JET

The "Injector jet," shown at Fig. 28, as made by Messrs. Butcher, is constructed to combine the power of the mixture jet with the economy in gas of the blowthrough. It has the appearance of a mixture jet, and may be used as such when it is desired to get the greatest amount of light from it. Under ordinary circumstances one tube is connected with the oxygen cylinder, and the oxygen enters the mixing chamber under pressure, there to mix with coal gas entering through the other tube from the main.

Gas and Oil Lamps.-Acetylene gives a small and very actinic flame, but the intensity is not quite uniform over its entire surface. The candle-power of an ordinary double 



\section{Direct Colour Photomicrograph.}

Screen-plate duplicating process (Paget).

Segtion of Quartz Felsite $\times 10$, under polarised light.
Objective ... ... Zeiss $50 \mathrm{~mm}$. Planar F. 4.5 .
Ocular ... ... None.
Condenser ... Back lens of Watson's Universal, with polariser and Selenite fitted underneath.
Illuminant ... Liliput 4 amp. arc.
Aux. Condenser Double convex lens, giving parallel rays.
Screen $\quad \ldots \quad \ldots$ Wratten \& Wainwright's $\mathrm{K}_{1}$
Plate $\quad \ldots \quad \ldots$ Paget colour plate.
Exposure ... ... 1 sec.

The specimen shows phenocrysts of quartz, more or less corroded at their edges, set in a microcrystalline ground mass of quartz and felspar. The darker parts point to the presence of ferro-magnesian minerals.

A chip from the rock is ground down and polished until extremely thin to produce the transparent preparation. 


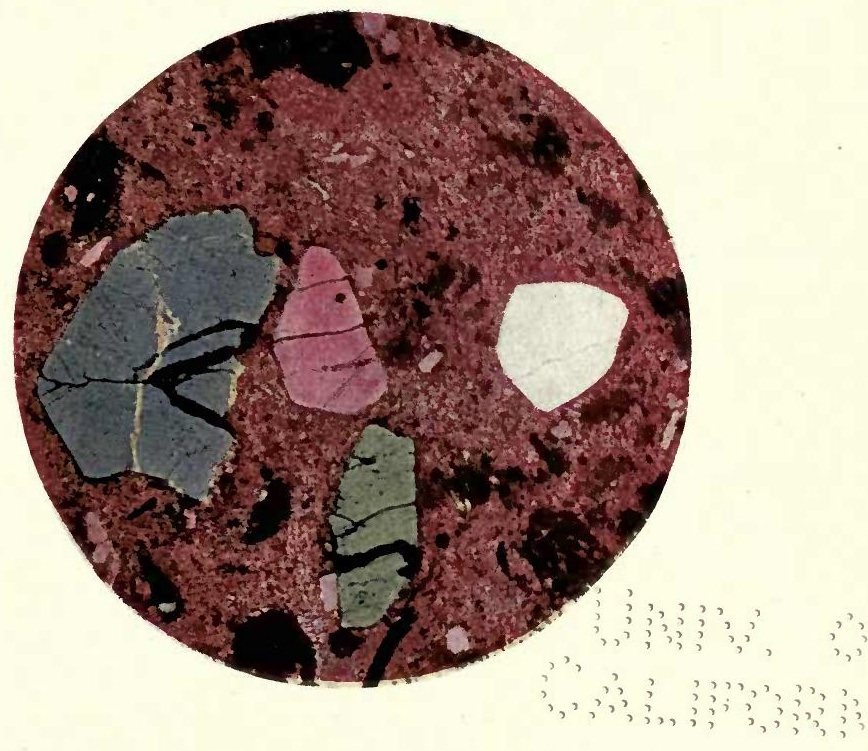



burner is about 80 . The preparation of the gas is seldom free from objectionable smell, but with good forms of automatic generators and pure carbide this is very much reduced, and no trouble or danger need be anticipated. It is a good plan to have a "purifier" connected up between the generator and jet, as it materially increases the brightness of the light. The purifier is merely a box containing pieces of asbestos on which water is deposited

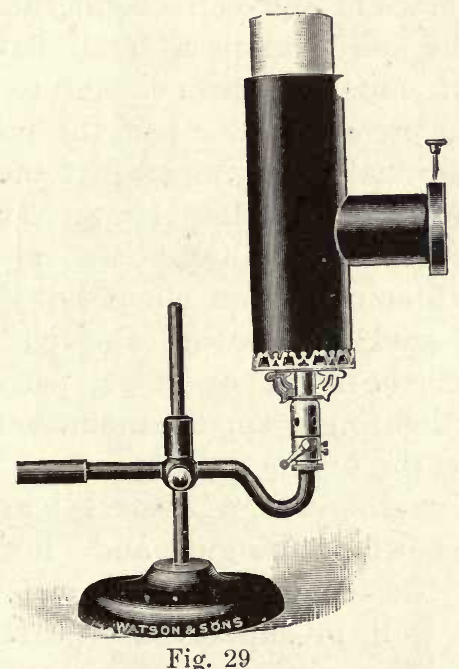

INCANDESCENT GAS LAMP

from the gas ; it may form part of the tray which carries the jet.

Incandescent Gas.-Incandescent gas is probably the most largely used of any illuminant, and possesses many advantages as regards simplicity and convenience. The chief objection to it is the difficulty of getting critical illumination without the formation of an image of the mantle. For work other than critical it is quite efficient, although not very intense ; the inverted form is generally to be preferred. Fig. 29 illustrates a convenient vertical 
burner; the chimney carries an iris diaphragm whose aperture may be used as the source of light and focussed instead of the mantle, in order to eliminate an image of the structure of the mantle in the field of view.

Incandescent Spirit, or Petrol Lamps.-When gas is not available a mantle may be heated by a flame from the vapour of methylated spirit or petrol, which is forced through a small jet, and mixing with air in the burner forms a very efficient and convenient substitute for gas. In some of the spirit lamps a small flame is used to keep the spirit container warm enough to give off vapour under sufficient pressure ; in others the pressure is maintained by occasionally blowing air into the reservoir from a rubber bulb, as in the Meta lamp. The petrol lamps require neither of these alternatives, as the vapour is given off continuously when once the jet is hot. The lamps are started by heating the jet, or small pipe through which the spirit or petrol passes, by a spirit flame, or by lighting a small amount of spirit placed in a cup below the burner.

The "Perfectol" and "Invetrol" are good petrol vapour lamps with upright and inverted mantles respectively. The simplest models can be hung from a nail on the wall, or supported on a stand, as shown in Fig. 46. Whenever petrol, methylated spirit, or even paraffin are used for burning purposes there is danger of fire, but with proper usage the vapour lamps are perfectly safe and they give a brighter light than incandescent gas. The small petrol lamp shown at Fig. 46 gives about 50 c.p., while larger ones give from 100 to 300 c.p. The chief difficulty is the heating up previous to lighting. This takes two or three minutes, and care must be taken that the jet is thoroughly hot. On this account a lamp fitted with a bye-pass is greatly to be preferred, so that the light can be turned off between exposures. The larger Perfectol lamps are so fitted, and 
they are also carried on stands with clamp for adjustment of height on a vertical pillar. Care must also be taken to keep the jet clean; a needle, to ensure this, forms part of the burner.

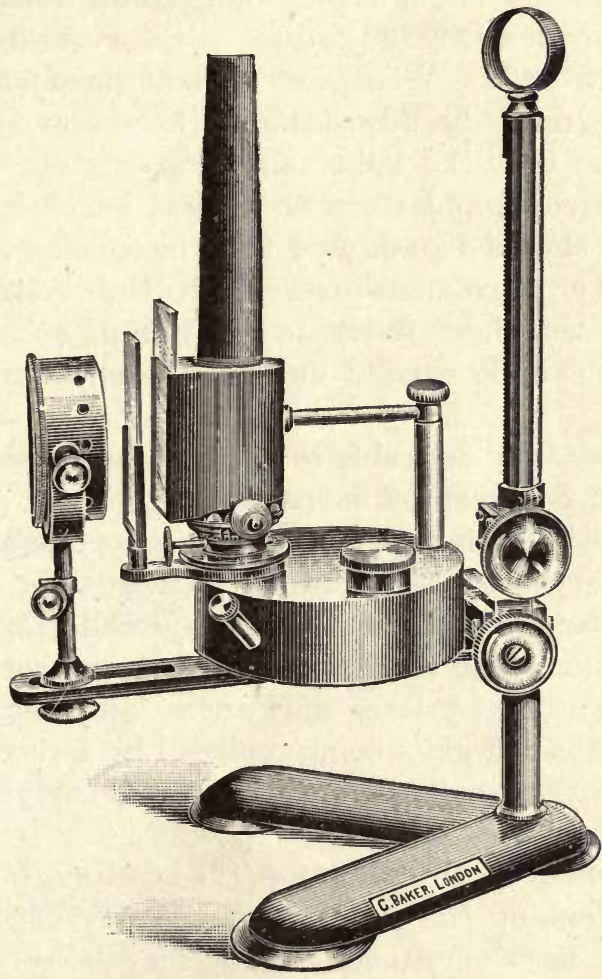

Fig. 30

OIL LAMP WITH ADJUSTABLE CONDENSER AND SCREENS

Oil Lamps.-Even now, with all the advantages attending more intense illuminants, the oil lamp still holds a prominent place. And, apart from its lack of brilliance, it is as good as any illuminant for photomicrography. A flat flame is always used, and when its edge is turned 
towards the microscope critical illumination is easily obtained. The light is improved by dissolving a little camphor in the paraffin, and if the wick is properly trimmed, and the condenser correctly adjusted, it is quite brilliant enough for high-power work. A good form is shown at Fig. 30 .

Lamp Stands.-Whatever radiant is made use of, the lamp must be adjustable in height and from side to side, so that the light can be accurately centred to the microscope, and when an optical bench is employed the lamp should be adapted to it in some suitable way. It is very convenient to attach the collecting lens and also the light filters to the lamp, as in Fig. 30, but they can be carried on independent stands if preferred.

It is also very desirable to enclose the radiant in a box or lantern to screen off extraneous light, and a specially made one is often supplied with large cameras (Fig. 3). An ordinary projection-lantern with lens and condenser removed answers the purpose very well (Fig. 2), or a metal chimney or shade can be made to surround the light (Fig. 29). A large dark-room lamp may even be adapted, the red glass being replaced by a sheet of metal pierced with a suitable aperture, and carrying an iris diaphragm if possible.

Selection of an Illuminant.-The advantages and drawbacks attending the use of the majority of illuminants have now been mentioned, but it may be convenient to summarise the points on which the selection of one or another mainly depend. Sunlight may be left out of consideration, and the choice made from the artificial illuminants. The decision usually turns on the availability of electricity or gas, and the question of expense. Electric light is no doubt the most convenient, and should be chosen whenever possible.

When direct current is available the selection should 
be made in the following order : Automatic arc, hand-feed arc, Nernst lamp.

For alternating current: Nernst lamp, which may be: supplemented when a more powerful light is required by a hand-feed arc.

In the absence of the electric current : Limelight, incandescent gas or vapour lamps, oil, acetylene; the oil lamp taking first place for strictly critical work not requiring a very intense light.

At present mercury vapour lamps hardly take the place to which their good features would entitle them, on account of their fragility and a lower intensity than that of are or limelight, but improvements may at some future time place them quite in the forefront of artificial illuminants.

Approximate power of Illuminants.-The following table may be of service in the selection of an illuminant as showing the candle-power given by some of the most common. The figures are only approximate, and vary in each case according to the particular conditions, but they give a rough idea of the comparative exposures necessary.

Flat-wick oil lamp .

Candle-power.

Incandescent gas

15

Acetylene

$35-60$

Spirit and petrol vapour lamps

40

1-ampère Nernst, 200 volt

$50-300$

Blow-through jet

200

400

Injector jet

1000

Mixture jet

$1000-2000$

4-ampère hand-feed are

300-500

5-ampère automatic are

1000

20-ampère automatic are

2500

35-ampère automatic arc

4000

75-ampère automatic are

10,000 


\title{
CHAPTER V
}

\author{
CONDENSERS
}

THE optical part of the illuminating system consists of one or more lenses, used, as their name implies, to condense the light from the radiant, and illuminate a small area very intensely. The complete system is made up of two separate units, "the substage condenser " and the "collecting lenses." The latter are also known as "auxiliary condensers," "parallelisers," or "bench condensers," denoting that their function is to collect the light from the radiant, and project it on to the substage condenser, or that they are used to project a parallel beam of light, and that they form part of the optical bench rather than of the microscope. The function of the substage condenser is the illumination of the object by a cone of light whose angle is comparable with that of the objective in use. In this chapter the types of lenses available for these purposes are described; a discussion of the manner in which they are used being left over to succeeding sections.

Diaphragms and light filters are also necessary items in the illuminating system, as strict control over the amount of light, the angle of the illuminating cone, the illuminated area, and the constitution of the light, are essential to success in photomicrography.

Aplanatic Cone of a Condenser.-The simplest condensers are uncorrected double-convex and plano-convex lenses, or partially corrected combinations of two or three lenses, but there are others in which the spherical and 
chromatic errors are corrected with an accuracy approaching that of objectives. Parallel rays passing through different zones of an imperfectly corrected condenser are not focussed to the same point, but there is a larger or smaller area around its centre from which the rays focus very closely to one point. The cone made by these rays is called the "aplanatic cone" of the condenser, and it increases in proportion as the spherical errors of the condenser are eliminated, until rays from all zones focus to one point, when its aplanatic cone equals its numerical aperture. At the apex of this cone all the available light is brought to a focus, whereas at no other point within or without the cone is this the case. As there is no such definite point with an uncorrected condenser, it cannot utilise the full power of the radiant so efficiently, and the exposure that even a good one necessitates may be as much as one-third greater than is needed when a corrected condenser of the same aperture is used. Besides giving a cone that is far from aplanatic, uncorrected condensers are not achromatic, and they usually produce colour-fringes round the disc of light they project, and the outer zones of any area on which the light falls are therefore very imperfectly illuminated.

Power of a Condenser. - Condensers are made of varying focal length according to the use for which they are intended, and the size of the image of the radiant that they form increases with the focal length. The focal length of the condenser used must be sufficient to ensure an even illumination of the entire area of the aperture of the substage condenser, or field of the objective, as the case may be. On the other hand, the N.A. and intensity of illumination increase as the focal length decreases, and these must be as high as possible, particularly in the case of the substage condenser, as the N.A. of the objective cannot be fully utilised unless that of the illuminating cone is adequate. The power of the condenser must 
consequently be adequate to give the illumination required by the objective, and increase as the power of the latter increases and its field of view decreases.

Bull's-eye Condensers. - The most commonly used condenser is a plano-convex uncorrected lens known as a

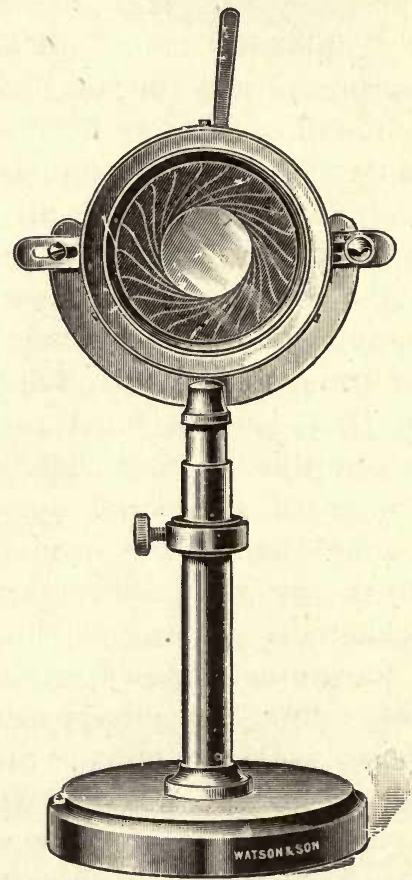

Fig. 31

BULL'S-EYE CONDENSER WITH IIIS ON ADJUSTABLE STAND

"bull's-eye." Some of the cheaper forms have such large errors that they cannot be satisfactorily used for photomicrography, but the better ones can be used quite well for most purposes. Their spherical error is least when the convex side faces the parallel beam, or cone of light of smaller angle. That is, a bull's-eye is placed with its flat side towards a radiant at its focus when a parallel 
beam of light is required, or with the convex side towards the incident light when it is used to converge a parallel beam.

A double convex lens is also frequently used as a condenser. Suitable sizes are $2^{\prime \prime}$ to $4 \frac{1}{2}$ " in diameter, with focal lengths between $3^{\prime \prime}$ and $5^{\prime \prime}$. The Köhler lenses of Messrs. Zeiss, of this type, are specially constructed for condensing purposes. Their use is described on pages 134 and 150 .

Corrected Auxiliary Condensers.-Many workers consider that it is necessary to have the auxiliary condenser corrected with very considerable accuracy, and Messrs. Watson supply an achromatised aplanatic combination, computed by Mr. Conrady, and shown in Fig. 3. The lens is of very short focal length, and consequently has to be placed very close to the radiant to give a parallel beam of light; glycerin is therefore used between the component lenses instead of balsam, which would melt; and to prevent bubbles forming in the glycerin it is essential to use a cooling trough between the condenser and any light source that gives much heat. Although its diameter is only $2^{\prime \prime}$ it gives a much more intense illumination than a considerably larger uncorrected lens.

Aplanatic Condensers.-Intermediate between this very accurate lens and the simpler condensers lie the aplanatic condensers now so largely used, and of which the doublet designed by Mr. Nelson (Fig. 32), is a wellknown example. These are also small lenses of about $2^{\prime \prime}$ diameter, and although not perfectly aplanatic they give very good results; as there is no liquid or balsam between the component lenses a cooling trough need not be used to protect them. In our experience the chief advantage presented by the highly corrected condensers over simpler but well-made types, lies in the shorter exposures they allow, and this is sometimes an important consideration. The photo- 
micrographs obtained only show superiority in exceptional cases over those obtained when good single lenses are employed. The introduction by Messrs. Zeiss of a small aplanatic condenser in place of their older simple

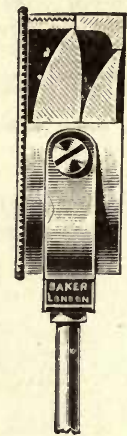

Fig. 32

NEISON'S APLANATIC CONDENSEl:

lenses is, however, evidence of the growing popularity of that form of condenser, and the purchase of one of the small aplanatic doublets is certainly to be advised. Failing that, a double convex lens of about $4^{\prime \prime}$ diameter, such as that supplied by Leitz, and shown in Fig. 7, should be obtained.

Condenser Mounts.-Any of these condensers can be obtained mounted on a suitable stand for the optical bench, and fitted with centring screws. Some of the simpler stands have only a vertical motion, others a horizontal as well (Fig. 31). An iris diaphragm is desirable on the optical bench, and is either fitted to the condenser or carried on an independent centring stand.

Flasks as Condensers. - Spherical flasks about 6 " in diameter, filled with water (Fig. 27), may be used in place of a bull's-eye, and have the advantage that by the addition of suitable colouring matters to the water the use of separate colour-filters is obviated, but they are neither so satisfactory nor so convenient as lenses. 
Substage Condensers.-Substage Condensers are usually combinations of two or more lenses, somewhat similar in construction to objectives, but made to concentrate light upon the object through the thickness of a slide.

Chromatic Condensers-The Spectacle Lens.-The simplest substage condensers are double convex lenses, such as the "Spectacle" lenses of Zeiss, and are very suitable for use with the small photographic lenses of 2 " or 3 " focus. Occasionally an even larger lens than these-about $2^{\prime \prime}$ in diameter-is useful to illuminate very large preparations.

Abbe Condenser.-The chromatic condenser of Abbe

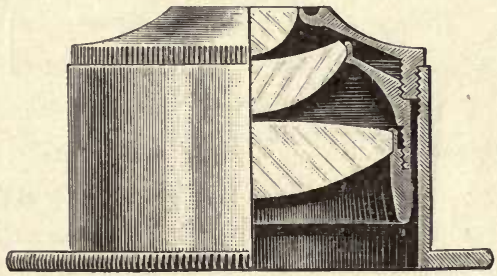

Fig. 33

ABBE CONDENSER

still maintains its position as the most frequently used substage illuminating apparatus, and as made by firms of repute it is a very efficient condenser, although far from aplanatic-its aplanatic cone is at the most $0 \cdot 65$-and only corrected for one colour. It is supplied in two forms, one with two lenses and a N.A. of $1 \cdot 0$ to $1 \cdot 2$, and the other with three lenses and a N.A. of $1 \cdot 3$ to $1 \cdot 4$ (Fig. 33). The latter should be oiled to the slide when used with oilimmersion objectives; and with its top lens removed has a focal length of about $\frac{1}{3}$, or $\frac{2}{3}$ ", forming a suitable illuminator for low-power objectives.

This type of condenser is fitted to most microscopes, as more accurate corrections than those it embodies are unnecessary for many purposes, but the superiority 
of achromatic and aplanatic condensers for critical work is now so generally recognised that quite a large series of corrected condensers is made, among which is the Abbe achromatic condenser of N.A. 1·0. It has a focal length of $\frac{1}{2}$, and an aplanatic aperture of 0.65 ; with front lens removed its power is reduced to $1^{\prime \prime}$ and its aplanatic cone to $0 \cdot 3$.

A planatic and Achromatic Substage Condensers are made of the following types :-

\begin{tabular}{|c|c|c|c|c|c|}
\hline & & \multirow[b]{2}{*}{$\begin{array}{l}\text { Focal } \\
\text { length. }\end{array}$} & \multirow[b]{2}{*}{ N.A. } & \multicolumn{2}{|c|}{$\begin{array}{l}\text { With front combina- } \\
\text { tion removed. }\end{array}$} \\
\hline & Type of Condenser. & & & $\begin{array}{l}\text { Focal } \\
\text { length. }\end{array}$ & N.A. \\
\hline 1 & $\begin{array}{c}\text { Low - power il- } \\
\text { luminators }\end{array}$ & $2^{\prime \prime}$ & 0.4 & & \\
\hline 2 & Ditto. . . & $\frac{2^{\prime \prime}}{3}$ & $0 \cdot 5$ & & \\
\hline 3 & Dry condensers . & $\frac{2^{\prime \prime}}{5}$ & $1 \cdot 0$ & $1 \frac{1}{4} "$ & $0 \cdot 4$ \\
\hline 4 & Ditto . . & $\frac{1^{\prime \prime}}{4}$ & $1 \cdot 0$ & $\frac{2}{5} "$ & $0 \cdot 4$ \\
\hline j & $\begin{array}{l}\text { Oil-immersioncon- } \\
\text { densers. }\end{array}$ & $\frac{1}{4}^{\prime \prime}$ & $1 \cdot 3$ to $1 \cdot 4$ & $3^{3 \prime \prime}$ & $0 \cdot 6$ \\
\hline
\end{tabular}

All the above condensers should have an aplanatic cone nearly as great as their N.A.; that of the condensers of N.A. $1 \cdot 0$, for instance, is usually about $0 \cdot 9$ or 0.95 .

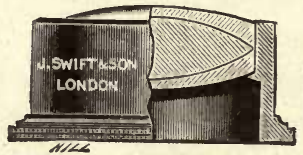

Fig. 34

LOW-POWER ILLUMINATOR BY SWIFT

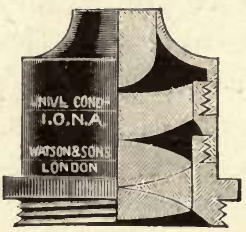

Fig. 35

ACH ROMATIC CONDENSEL, WATSON'S "UNIVELSAL" 
Such a complete series is only made by a few manufacturers, notably Swift, and Watson, others supply the two or three most generally useful, corresponding to Nos. 1, 3, and 5 .

They are intended for use in the following way :-

Low-Power Condensers.

No. 1 evenly illuminates objects up to about $1^{\prime \prime}$ in diameter for planar lenses.

No. 2 is to be used with low-power objectives up to about $\frac{1}{3}$.

Dry Condensers.

Nos. 3 and 4 have the same N.A., and most makers only provide one condenser of that aperture, but Swift, and Watson make two. Swift's "Paragon " and Watson's "Universal " represent No. 3, and have larger back lenses than the "Achromatic" and "Parachromatic" of the same makers corresponding to No. 4. The latter are of higher power and corrected with greater perfection, but require greater skill to use them to their best advantage ; they pass less light than the other two. These condensers are intended for use with medium- and high-power objectives, and with their front lens removed with low powers.

Oil-Immersion Condensers are for use with oil-immersion objectives, when the widest possible cone of light is



Fig. 36

APLANATIC OIL-IMMERSION CONDENSER BY LEITZ

required. A reference to Fig. 21 will show the advantage 
secured by the use of a film of oil between the condenser and the under side of the object-slip, permitting a ray $\mathrm{COK}$ to pass right through to the object without deflection. In the absence of oil a similar ray shown to the left of $\mathrm{AB}$ is totally reflected at $\mathrm{D}$, and the most oblique ray utilised is represented by EFG at a considerably smaller angle to the perpendicular. The highest possible N.A. that can be used with a dry condenser is 1.0 , while oil-immersion condensers are made with a N.A. of 1.3 or 1.4 and an aplanatic aperture nearly or quite as large.

Leitz oil-immersion condenser illustrated at Fig. 36 has a N.A. of $1 \cdot 4$, and by removal of one or more of the component lenses can be used as a medium- or low-power condenser as follows :-

\begin{tabular}{l|c|c|c}
\hline & N.A. & Focal length. & $\begin{array}{c}\text { Working } \\
\text { distance. }\end{array}$ \\
\hline $\begin{array}{l}\text { With top lens removed . } \\
\begin{array}{l}\text { With top, second and third } \\
\text { lenses removed . }\end{array}\end{array}$ & 0.64 & $20.3 \mathrm{~mm}$. & $8.5 \mathrm{~mm}$. \\
\hline
\end{tabular}

Apochromatic Condensers (Fig. 37).-Messrs. Swift, and Powell and Lealand, call the finest condensers they

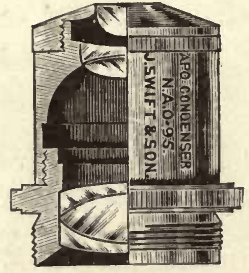

Fig. 37

SWIFT'S APOCHROMATIC CONDENSER

make "Apochromatic," as, by the employment of fluorite in their construction, they claim to achieve the corrections corresponding to that term. The front lens should never be removed from these as they are very 
sensitive to errors in centring of the lenses, and any alteration would be liable to upset the accuracy with which they were constructed.

Choice of a Condenser.- It is not at all easy to make selection of a condenser, when, as is so often the case, a single one has to be used for all purposes. It is generally best to get a moderately high-power condenser, as its front lens can be removed and the back combination then forms a useful low-power illuminator-with some sacrifice of correction however. There is considerable difference of opinion over the extent of the advantages presented by aplanatic and achromatic condensers over that of Abbe, apart from the shorter exposures required if the former are used; and if delicate work, done with an Abbe condenser, is compared with similar work with the more expensive corrected types, it is extremely difficult to find any difference. The superiority of the more highly corrected types is, however, now generally recognised as a factor in the production of a perfect image, and on that account it is certainly best to select one of them, but if cost is a material consideration an Abbe will serve very well.

Although the aplanatic aperture of an Abbe illuminator is, as a rule, only about 0.5 , this does not mean that it will restrict the N.A. of any of the high-power objectives that can be used with it to $0 \cdot 5$. A measure of the effect of the aperture of the condenser on the performance of the objective is given by the mean of the aplanatic apertures of the objective and condenser, and the figure is known as the working aperture. Thus an objective of N.A. 1·4 can only be used to its full advantage with an oil-immersion condenser having an aplanatic cone of $1 \cdot 4$. If an achromatic condenser of N.A. 1.0 and an aplanatic cone of 0.9 is used, the working aperture is reduced to $(1 \cdot 4+\cdot 9)$ $\div 2=1 \cdot 15$, while an Abbe condenser gives a working aperture of only $(1 \cdot 4+\cdot 5) \div 2=0.95$ with the same objec- 
tive. For most purposes the difference between the apertures 1.15 and 0.95 is unimportant, but the use of an Abbe condenser sets a limit to the performance of an objective that is rather low for critical work. A ${ }_{6}^{1 \prime \prime}$ objective of high N.A. ( 0.9 for instance) could be used at full aperture with an achromatic condenser, but an Abbe would restrict its working aperture to $(\cdot 9+\cdot 5) \div 2=0 \cdot 7$, which is a considerable drop.

If it is decided to be content with an Abbe condenser it is best to get that with the larger N.A., as it is adaptable to a wider range of objectives than the lower power; with front lens removed it is a very good low-power illuminator. But if an achromatic condenser is decided upon the choice is wider. The advantages of a large aperture in objectives have been described, and to use this aperture as fully as possible a large illuminating cone is required, but the cost of the oil-immersion condensers is so great that the worker who dces not aspire to such difficult feats as the resolution of Amphipleura into dots may be well content with one of the dry condensers. Those with a wide back lens and a N.A. of 1.0 are the most generally used. They constitute in themselves almost a complete battery; they can be used with highpower oil-immersicn objectives without great sacrifice of aperture, with dry objectives at their full aperture, and with low powers down to about $1 \frac{1}{2}$ " or $2^{\prime \prime}$. They are, in fact, practically universal condensers, and can be used for almost any work, even to illuminate such a large field as is covered by a short-focus planar lens. But they fail with the longer-focus planars, for which one of the low-power illuminators must be chosen, if a condenser is to be employed; and they are also inadequate for the photomicrography of many diatoms and other objects which call for the highest resolving power obtainable. Still, it is between these two extremes that by far the greatest amount of photomicrography is done, and for 


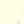




\section{Low-power Photomicrography.}

EмвRYo Сніск, 54 hours' incubation $\times 15$

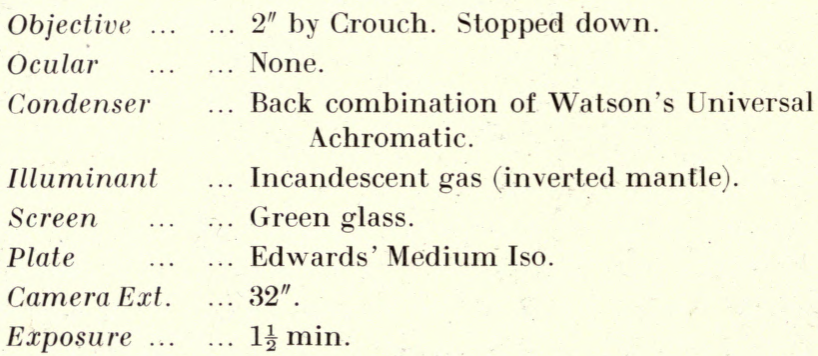

An example of low-power photomicrography with an achromatic objective, illuminated by means of the back combination of an achromatic condenser.

In order to obtain depth of focus the objective was stopped down by placing a circular metal diaphragm having a small central aperture between the two lenses of the objective combination.

The preparation was stained with carmalum, and, as many of the finer blood vessels in the clear area (area pellucida) were only faintly stained, a green pot glass screen was used to increase the contrast of the paler structures. This had the effect, however, of obscuring some of the detail in the more densely stained portions. 

that the dry achromatic condensers of N.A. $1 \cdot 0$ and aplanatic cone 0.9 are entirely suitable, and most workers will find this type the best for all-round use.

Objectives as Condensers.-It is possible to use objectives in place of condensers, and centring mounts with the R.M.S. screw are supplied for that purpose. Nothing, however, is gained by so using them, as, even if they are better corrected than condensers, their corrections are upset by making them work through a slip which is much thicker than the cover-glass for which they are adjusted. 


\section{CHAPTER VI}

\section{EXPERIMENTS ON ILLUMINATION}

Critical Light.-The correct illumination of an object is of the utmost importance when a high degree of resolu-

- tion is required, and the use of the condensers needs even more study than that of the objectives and eyepieces. The object to be attained is a strict correlation of the two optical parts of the microscope, the illuminating and the image-forming, and their exact combination in one complete optical system. This is the most difficult problem to be faced in photomicrography, but no high-class work can be done unless it is understood and practised. The following experiments are intended to pave the way to its complete mastery, and to serve as an introduction to the proper use of the radiant and condensers, and to the procedure by which the best illumination is obtained.

It will be well to start with a concrete conception of the problem, and for that purpose definitions of "Critical Image" and "Critical Illumination" are given in the words of Mr. E. M. Nelson, the protagonist of that method of illumination for microscopical research :-

Critical Image.- "The image of an object is critical when it is obtained by means of an objective of fine quality which has been placed in correct adjustment for that object by its screw collar, or by the alteration of the tube length, and when the illumination is critical."

Critical Illumination.- "An object is said to be illuminated critically when it is placed at the apex of a solid axial cone, the aperture of which is not less than 
three-quarters of the N.A. of the observing objective" (Journal of the Royal Microscopical Society, June, 1910).

Critical illumination is the condition under which an objective will work to its best advantage, and produce the finest image of which it is capable, but the procedure by which it is obtained may seem rather complex to a beginner, and is here led up to through simple experiments. The adjustments are really not difficult, and if the principles underlying them are studied at the outset and always put in practice they soon become almost mechanical, and the worker who has mastered them is in a position to tackle any kind of photomicrography, from the lowest to the highest power.

In the next chapter, dealing with the use of small photographic lenses, these principles seem to be abandoned. But there is then no question of great resolution, and the object field to be evenly illuminated is, as a rule, so great that the apex of the solid axial cone of light, if employed, would only appear as a bright spot in its centre. The difficulties introduced by the appearance of this bright spot or image of the radiant across the object are so great as to overshadow any gain produced by that method of illumination, and consequently another course is usually adopted under these circumstances. On the other hand, where ordinary microscopic objectives are used covering a much smaller field, critical light should always be aimed at, and means to employ it are generally to be found.

\section{Preliminary Experiments with a Condenser:}

1. Hold a bull's-eye condenser or double convex lens in such a position that it throws an image of a radiant of low intensity on to a white card. Remove the card and place the eye in the position of the aerial image, the entire disc of the condenser appears evenly filled with light.

2. Move the condenser into other positions between 
the eye and, lamp, and note that it is no longer full of light, but shows characteristic shadow areas on part of its disc.

3. Keeping the eye and condenser in their first positions, move the lamp out of the axis of the condenser, and notice that shadows appear on the limb of the bright disc.

4. Place the condenser in such a position that the light is at its focus; it projects a parallel beam of light, as is evidenced by moving the card to and fro, when it will be noted that the disc remains constant in size.

5. Move the condenser nearer to the radiant, it projects a divergent beam.

6. Move it further away, a convergent beam is formed; and by moving the card into a certain position an image of the radiant is focussed on it.

7. As the condenser is moved away from the radiant the card has to be moved nearer, and the image on it becomes smaller.

8. The disc projected by most condensers is not uniเorm over its entire surface, even when the light is small and placed correctly, but shows a central bright portion surrounded by a broader or narrower ring of shadow or colour. The central portion is all that is useful for illumination, and steps should always be taken to ensure that the colour fringes lie entirely outside the surface that has to be illuminated, or are cut off by means of an iris diaphragm.

9. Compare the disc projected from the same illuminant by corrected and uncorrected condensers of similar size and focal length. The increase in brilliance and the absence of colour fringes that follow the use of the corrected lens are noticeable.

Experiments with the Microscope.-These experiments can now be extended to the microscope, which is set up vertically or slightly inclined. A flat-wick oil lamp is the most suitable illuminant. It is placed with the edge of 
the wick, instead of the flat, facing the microscope at about $8^{\prime \prime}$ from the mirror.

1. The use of the Mirror.-The mirror has plane and concave surfaces, of which the plane should be employed whenever a condenser is used. To study the use of the concave mirror, place a $\frac{2 \text { " }}{3}$ or $1^{\prime \prime}$ objective and low eyepiece on the microscope, and take a suitable object. Remove the condenser and illuminate with the mirror only. It will be found that the concave gives a more intense light than the plane side, because the rays that it reflects are convergent instead of being parallel or divergent. The mirror should be capable of a vertical motion along the bar that carries it. Move it to or from the object until the position is found in which it gives the brightest possible light over a small portion of the preparation. The focus of the mirror, or the point at which it forms an image of the radiant, is then in the plane of the object. It may be remarked that on account of spherical aberration the mirror has no exact focus, but the region is fairly definite. The illumination under these conditions, though intense over a small area of the object, is of little use for photomicrography, on account of the image of the radiant which usually lies across the field of view, and it is necessary to raise the mirror until the object is evenly illuminated and the field just filled with light. If the mirror is raised any higher a diminution of light will be noticeable. This method of illumination is sometimes satisfactory with low powers, but it is never used when a suitable condenser is available.

2. To Centre the Mirror.-(a) Remove the objective and condenser, and elongate the draw-tube to its full extent. Look into the eyepiece from about $10^{\prime \prime}$ above it, a small circle formed by its diaphragm is seen in the centre of the eye-lens. Then move the mirror until an image of the light source is seen exactly in the centre of this circle. Use the flat side of the mirror. 
(b) Second method.-With the condenser, low-power objective, and eyepiece on the microscope. Rack the condenser and objective up or down until an image of the light can be seen; move the mirror until the image lies centrally across the field of view.

3. To centre the Condenser.-Close the iris.diaphragm below the condenser to a pin-point. Fit a low-power objective, $\frac{2}{3}$ " or $1 \frac{1}{2}^{\prime \prime}$, and low eyepiece, and endeavour to focus the edge of the aperture by racking either the microscope tube or condenser up or down. An Abbe condenser does not give a sharp image of the diaphragm, and when the iris is close beneath it, as in the standard Continental substage, it is praotically impossible to focus the image even with $2^{\prime \prime}$ or $3^{\prime \prime}$ objectives. An achromatic condenser gives a sharp image, and, with the iris further away from it, as is usual in the English substage, focus can be obtained with a $\frac{2}{3}$ " or higher objective. With the margin of the iris as nearly as possible in focus, centre the aperture by gently turning the adjusting screws on the condenser ring, or, if they are fitted to a nose-piece instead, centre the objective.

If there is no means of bringing the optical axes of the objective and condenser into alignment, and the latter appears to be out of centre, turn it round in its mount, and possibly a position will be found in which the centration is fairly accurate.

When it is impossible to focus the iris below an Abbe condenser with the objectives at hand, focus on to the upper surface of the condenser with a $1^{\prime \prime}$ or $\frac{2}{3}$ " objective, until a dimly-defined dark spot appears in the field, and about $\frac{1}{3}$ of its diameter. Centre this as above.

4. To Focus the Substage Condenser.-Use the plane mirror to reflect the light on to the condenser. Place a slide on the stage and focus with a $\frac{2}{3}$ or other low objective and low-power ocular. Then rack the condenser until an image of the radiant is visible across the 
object; when this is as sharp as possible the condenser is said to be focussed. If the image of the lamp-flame is not quite central a slight movement of the mirror will make it so.

5. The Appearance of the Back Lens of the Objective.The back lens of the objective should be examined by removing the eyepiece; or its image, formed by the eyepiece, called the "Ramsden circle," may be examined by a magnifying glass held slightly above the eye lens. The appearances observed can be compared with the results of the first set of experiments with a bull's-eye, and they are most important as indicating whether the whole of the N.A. of the objective is being utilised or not. They are as follows :-

(a) When the condenser is focussed the back lens of the objective, or the Ramsden circle, is filled with light if the N.A. of the condenser is large enough.

(b) When the condenser is racked down from its focussed position shadows appear round the Ramsden circle, and the full N.A. of the objective is not used.

(c) When the condenser is racked up shadows appear towards the centre of the circle, and the entire aperture is again not used.

(d) When the iris diaphragm is closed down a black ring appears round the Ramsden circle, and the amount by which the aperture of the objective is restricted can be estimated by comparing the breadth of this ring with the radius of the illuminated part of the circle.

(e) When the condenser is racked down until the iris is in focus, and the diaphragm then opened, an image of the radiant can be seen in the Ramsden circle.

Errors in centration of the lamp, mirror, or condenser can be detected by the above examinations of the Ramsden circle.

6. The Appearance of the Object Field.-Having made the adjustments described to centre the condenser, and 
to focus an image of the radiant across the centre of the object, which is simultaneously in focus, and made sure by examination of the back lens of the objective that three-quarters or more of its N.A. is in use, the conditions laid down by Mr. Nelson as governing the production of the critical image have been attained. But the appearance of the object itself, with the image of the lamp-flame forming a bright streak across it, is not suitable for photomicrography. The part illuminated is shown to the best advantage possible, but some means must be taken to equalise the light over the whole field before a useful photomicrograph can be taken, and the means by which this is done, without depreciating the image, are most important.

7. Effect on the Field of View of Moving the Condenser.As the condenser is racked above or below its focus notice that the illumination over the field is reduced, and an effect similar to closing down the iris is observable on the back lens of the objective. But it is often possible by a very small movement from exact focus to equalise the light over the field, and do away with the image of the radiant, without any apparent restriction of the N.A. of the objective.

8. The Relation of the Focal Length of the Condenser to the Power of the Objective.-(a) Still keeping the $\frac{2}{3}$ " objective in position, remove the front lens of the condenser, or replace the original condenser by one of longer focal length. Note that the remaining combination must be racked down to form a sharp image of the radiant across the object, and also that the image is larger than that formed by the complete condenser. It may cover the entire object, or fill the field of the objective.

(b) Focus the complete condenser again, and replace the low objective by a higher power, a $\frac{1}{6}$ " for instance. The entire field is now probably covered evenly by the image of the light. 



\section{Some faults commonly met with.}

\section{Diffraction.}

A and B Hains on tie Wing of Honey Bee $\times 186$

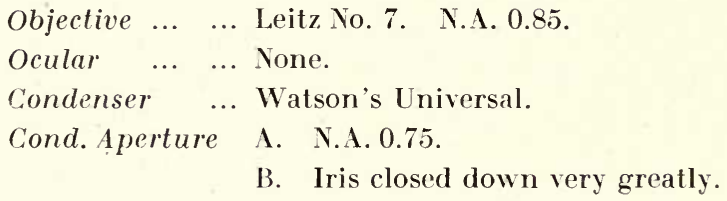

A The hairs are sharply defined, but owing to the small penetration of the objective at the large aperture, the bases of the hairs, and hairs in other planes are out of focus.

B Restriction of the aperture of the condenser causes white line diffraction effects and duplication of the points of the hairs. The penetration of the objective is greatly increased at the low N.A., and hairs previously out of focus become visible.

D Diнтом-Navicula crabro $\times 400$

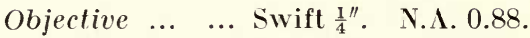

Ocular $\quad \ldots . . .$. Swift No. 2.

Condenser ... Watson's Universal.

Cond. Aperture The print is composite from two negatives, the left half being taken at nearly open aperture (N.A. 0.75), the right with iris greatly closed.

Diffraction effects are visible as a series of concentric lines round the diatom, and specks of dust invisible with the open aperture become apparent as small circles when the N.A. is restricted.

\section{Flare Spot.}

C Group of Diatoms $\times 60$

The white flare spot in the centre is due to reflections from bright portions of the interior of the microscope tube, no eye-piece being used.

Faulty definition, and lack or excess of contrast, due to the use of unsuitable plates or colour screens, are illustrated on Plates 17 to 21 . 


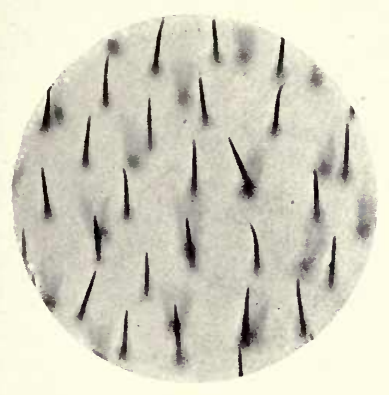

A
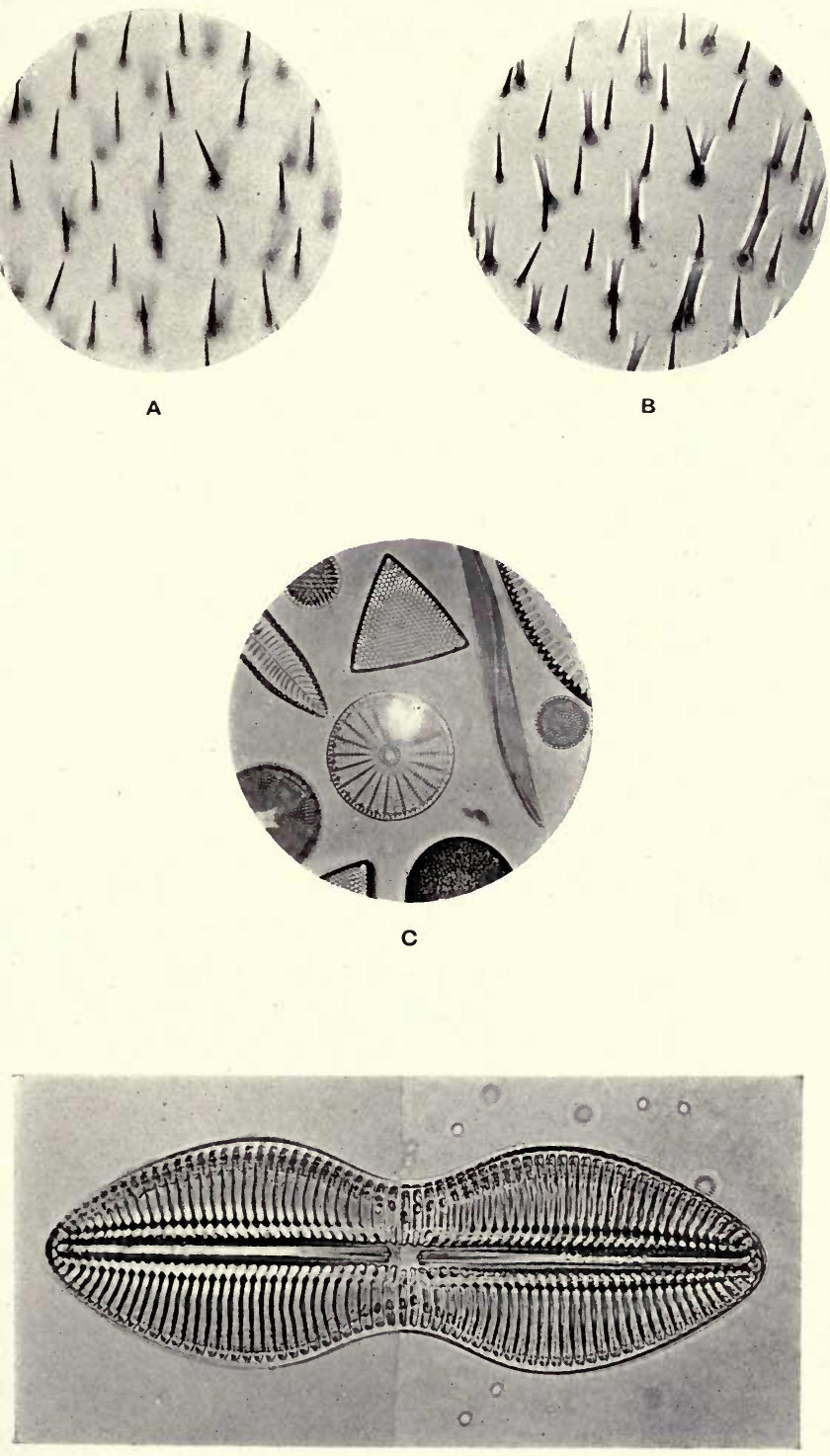

It follows that a condenser must be selected to suit the objective in use. A low-power objective requires a condenser of correspondingly long focal length.

9. Numerical Aperture of the Condenser and Objective. -Focus the image of the light across the object by means of the back combination of the condenser, and fit the $\frac{1}{6}$ " objective. Examine the Ramsden circle; it will be found impossible to fill the back lens of the objective under these conditions, and the light employed is not critical. It may be that no inferiority in the image to that obtained with critical light can be detected; it is only a worker of experience examining special objects who can detect the depreciation, and for a great deal of photomicrography critical light is not essential, but the experiment points to the necessity of having a condenser whose N.A. is adequate for the objective in use. It is generally not desirable to use the entire N.A. of the objective, as very few lenses work quite satisfactorily without a small restriction, but whatever aperture can be used, or is best for the particular object under examination, should be evenly filled with light.

10. Diffraction Effects and N.A.-Examine carefully an object with well-defined lines or dots-an insect with hairs, for instance-first with the iris open, and then gradually closing it down. When the iris is wide open the image appears drowned in light and indistinct, as the aperture is closed to $\frac{3}{4}$ or $\frac{2}{3}$ the clearest image is formed, and as the iris is further closed to $\frac{1}{2}$ or $\frac{1}{4}$ aperture "diffraction effects" are set up, and appear as white margins to lines or dots or as double images (Plate 12). As a rule, these become apparent in a photomicrograph before the iris is closed far enough to make them very noticeable visually, and great care must always be taken to avoid them. Different objects require a varying restriction of aperture. Stained bacteria are best shown with as little curtailment of the aperture as possible, 
while living micro-organisms are only shown up in sufficient contrast to the field when the aperture is closed to about $\frac{1}{2}$ or $\frac{1}{33}$; diffraction is necessary to give them distinctness, but it is very liable to be overdone in the photomicrograph. Oil-immersion lenses can be used with a smaller restriction of aperture than dry lenses, and apochromats than achromats.

11. Depth of Focus and Aperture.-Examine a fairly thick object (e.g. a large diatom), and note that as the iris is closed a greater number of planes come into focus at once, until diffraction interferes with the result. To get as great a depth of focus as possible it is better to replace the objective by one of lower N.A. rather than restrict the aperture of the higher, or to use a lower power objective and longer extension to get the same magnification.

12. Critical Illumination with Various Radiants.-Obtain critical light with whatever radiants are available, using blackened glass to protect the eye from the glare of any that are very intense, and examine the field of view and the Ramsden circle in each case when using a moderate objective (e.g. $\left.\frac{1}{6}{ }^{\prime \prime}\right)$. It will be found that only a few radiants evenly fill the back lens of the objective. The edge of the oil-lamp flame, a Nernst filament in some cases, and a mercury vapour lamp, are satisfactory. Structure can usually be made out in the image of an arc or limelight, and incandescent mantles always give a network over the field. An ordinary electric lamp is worse than any, showing its filament across the object, and it does not fill the back lens.

13. Introduce a piece of Ground Glass between the Radiant and Microscope.-A diffused light is obtained, and the field becomes evenly illuminated. If the surface of the ground glass is focussed as the source of light the image is covered with a granulation. Ground glass is never used when critical illumination is required. 
14. Use the Flat of the Oil Flame.-The field of view is evenly covered, and so far as can be judged the back lens of the objective is filled with light, but such a large light source is never of even intensity over its surface. And the condition for critical illumination is far from attained ; instead of a solid axial cone there are many cones, both axial and oblique, overlapping.

An Evenly Illuminated Field with Critical Light.-The process adopted to obtain critical light has now been outlined, and the consequent appearance of the object noted. In certain circumstances, particularly with highpower objectives, the best conditions of illumination for photomicrography are attained, but more frequently an image of the radiant quite precludes the possibility of taking a photomicrograph, and it is necessary to consider how the illumination may be made even over the entire field, without destroying the perfection of the image. The use of a larger radiant surface and diffused light have been mentioned, and their inapplicability to critical illumination pointed out. There are, however, two courses that may be pursued to get even light, without in any way depreciating the image formed.

The first is based on a slight departure from absolute focus of the image of the radiant.

The second depends on the amplification of that image by means of an auxiliary condenser, or the replacement of the image of the radiant by that of the illuminated disc of the auxiliary condenser.

Slight Departure from Strict Focus of the Radiant.Exp. 7 has shown that an evenly illuminated field may be obtained by racking the substage condenser up or down from its position of focus, and that, in many cases, this can be brought about by a very slight movement, involving no curtailment of the aperture of the objective. Many experienced workers are of opinion that no loss of definition results from a slight upward motion of the 
condenser, provided the back lens of the objective remains full of light, but the method should only be used with caution, and an examination of the back lens of the objective never omitted in critical work under these conditions.

Use of the Auxiliary Condenser.-The use of the auxiliary condenser is most readily studied with the microscope in a horizontal position on the optical bench, employing a piece of white card on which to focus the light.

Magnified Image of the Radiant.-Place a bull's-eye or double convex lens $2^{\prime \prime}$ to $4^{\prime \prime}$ in diameter in such a position that it projects a magnified image of the radiant across the diaphragm of the substage. Fig. 38 shows in dia-

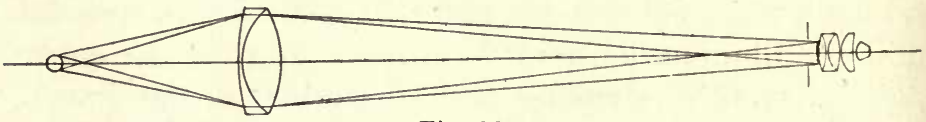

Fig. 38

DIAGRAM OF ILLUMINATION WITH AUXILIARY CONDENSER

grammatic form the course of the rays when a WatsonConrady condenser and Nernst lamp are used. The condenser is placed about $3 \frac{1}{2}$ " from the lamp, in such a position as to project a sufficiently large image of the radiant to cover the maximum aperture of the substage condenser that it is desired to make use of. The substage condenser is now in the position of the eye in the first experiment with the condenser page 115. The bull's-eye is full of light, and may be considered as the source of light to be focussed by the substage condenser across the image plane. This is effected either by holding a pencil point against its surface and focussing it by the substage condenser, or by closing down the attached iris, if there is one, until the aperture is so small that it can be focussed in the object plane. If the auxiliary condenser is suitable and properly centred the field of view should be evenly illuminated, and an examination of the 
Ramsden circle should show the back lens of the objective full of light. But if the image of the radiant is not sufficiently large to cover the back lens of the substage condenser, the full aperture of the latter is not made use of, and the effect produced by a partially closed substage iris may be noted on the back lens of the objective. If black shadows appear on the Ramsden circle their cause may be found in bad centring of the bull's-eye, or in its spherical aberrations. Any falling off in brightness towards the edges of the image or disc of light projected, has the same effect on the aperture of the substage condenser as a disc of light too small to cover its back lens or an iris diaphragm restricting it.

The next diagram, Fig. 39, is intended to demonstrate

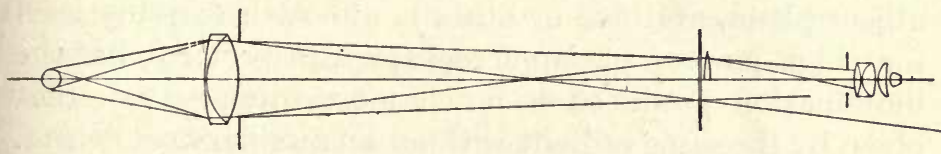

Fig. 39

DIAGRAM OF ILLUMINATION BY MEANS OF TWO AUXILIARY CONDENSERS

a slight weakness inherent in this method of illumination. A second single condensing lens is shown close behind an iris diaphragm in the upper part of the figure. The lower half indicates the course of the pencils of rays when this secondary lens is not introduced, and if the substage condenser is imagined as situated in the position of the secondary lens (the conditions of Fig. 38), and if its full aperture is to be employed, the diagram shows that the outer parts of the image of the radiant covering it are largely ineffective, owing to the pencils of light forming them, more or less missing the substage condenser. On the other hand, when the auxiliary lens is introduced, as shown in the upper part, all the light is directed into the substage condenser.

- Parallel Light.-A beam of parallel light, obtained by placing the radiant at the focus of the condenser, may be 
employed in place of the convergent beam just described. The condenser is focussed just as before, and the following conditions must be complied with. The beam of light must be sufficiently broad to cover the entire back lens of the substage condenser, or such part of its aperture as is made use of, and any colour fringes round the disc of light must fall right beyond the condenser for the reason above mentioned. Such a falling off of light at the edges often occurs when an uncorrected condenser is used, and may effectually prevent the utilisation of the full aperture of an oil-immersion substage condenser.

Either of the methods described above gives an image that suffers in no respect in comparison with that obtained when an image of the radiant is focussed in the object plane, and one or other is almost invariably used for high-power photomicrography, especially as the illumination produced is much more intense than that given by the same radiant without an auxiliary condenser.

The Auxiliary Iris Diaphragm.-The importance of the auxiliary iris is brought out by the above experiments, and it should always form part of the optical bench equipment. When closed down it is used for focussing and centring purposes. It is also used for cutting off the outer zones of the beam projected by the condenser, as only enough light should be used to cover the aperture of the substage condenser, any more is liable to give rise to reflections and mistiness of image. Its aperture is adjusted, after focussing the substage condenser, by examining the image and slowly opening the diaphragm until its edges coincide with the limits of the field of the objective. 


\section{CHAPTER VII}

LOW-POWER PHOTOMICROGRAPHY

Рнотомicrography strictly refers to the photography of objects so minute that a microscope is necessary to amplify them sufficiently to get a useful picture, but the boundary line between objects that do, and do not require a microscope is rather indefinite, and depends to some extent upon convenience, and upon the apparatus available. Magnifications ranging up to about 12 diameters can be easily attained with photographic lenses of small focal length, so this survey of the processes of photomicrography is opened with a description of some of the methods employed when these lenses are used. It is generally the simplest plan to attach the lens to the camera and dispense with the microscope; the latter, however, forms such a convenient object- and lens-holder that it may be preferred to make use of it merely for that reason.

The very simple arrangement to be first described is useful for reduction in size as well as magnification, the only alteration being a reversal of the relative distances between object and lens, and lens and plate. The apparatus is also suitable for making enlarged prints and lantern slides from negatives.

Opaque Objects. - Natural history objects of various kinds, small works of art, and so forth, may be photographed in the manner illustrated in plan by Fig. 40. A piece of white paper or card is bent as shown and an 
illuminant is placed in each of the two folds. The folds of the paper shield both camera and operator from direct light, and also act as a capital reflector. The background may be white or coloured paper as desired. To avoid shadows the object is fixed by plasticine or other adhesive to a piece of plate glass supported some distance from the background. If illumination of the object from one side is required one or other of the lights is dispensed with or lowered, and in this way the amount of shadow required to give the necessary relief can be obtained at will. To make a permanent apparatus the

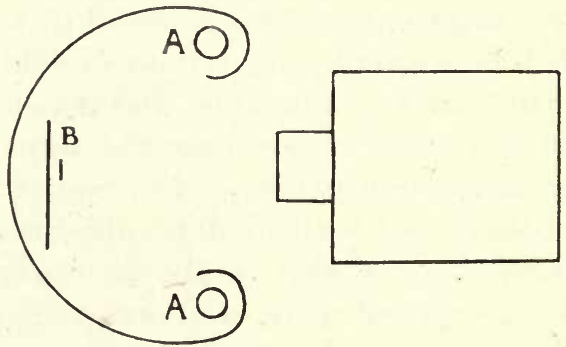

Fig. 40

DIAGRAM OF REFLECTING ILLUMINATOP

reflector is placed in a box fitted with lamp chimneys if gas is used for illumination. Grooves or slides of similar design to those used for the cameras shown at Figs. 1 and 2 can be made to carry the object-holder, and the back of the box is made removable in order that the holder may be moved backward or forward, or the object adjusted. The reflector is not continuous, the background is attached to the back of the box and the lamp reflectors are made of thin sheet metal, either bright or lined with white paper. The front or lid of the box, which is hinged or slides in a groove, is pierced with a central aperture, about $1 \frac{1}{2}$ " in diameter, and two pieces of clock-spring are screwed on to it to hold transparent preparations. Electric light 



\section{Low-power Photomicrography.}

Dog F $F_{\text {LEA }} \times 40$

Objective ... ... Zeiss Micro-planar. $25 \mathrm{~mm}$.

Condenser .. Watson's Universal, back combination.

Screen _.. ... Wratten's B and G (yellow-green), 5100-6000.

Illuminarit ... Liliput arc 4 amps.

Aux. Condenser Biconvex lens, parallel light.

Plate $\quad \ldots \quad \ldots$ Imperial N.F.

Exposure $\ldots \quad \ldots \frac{1}{2}$ sec. 


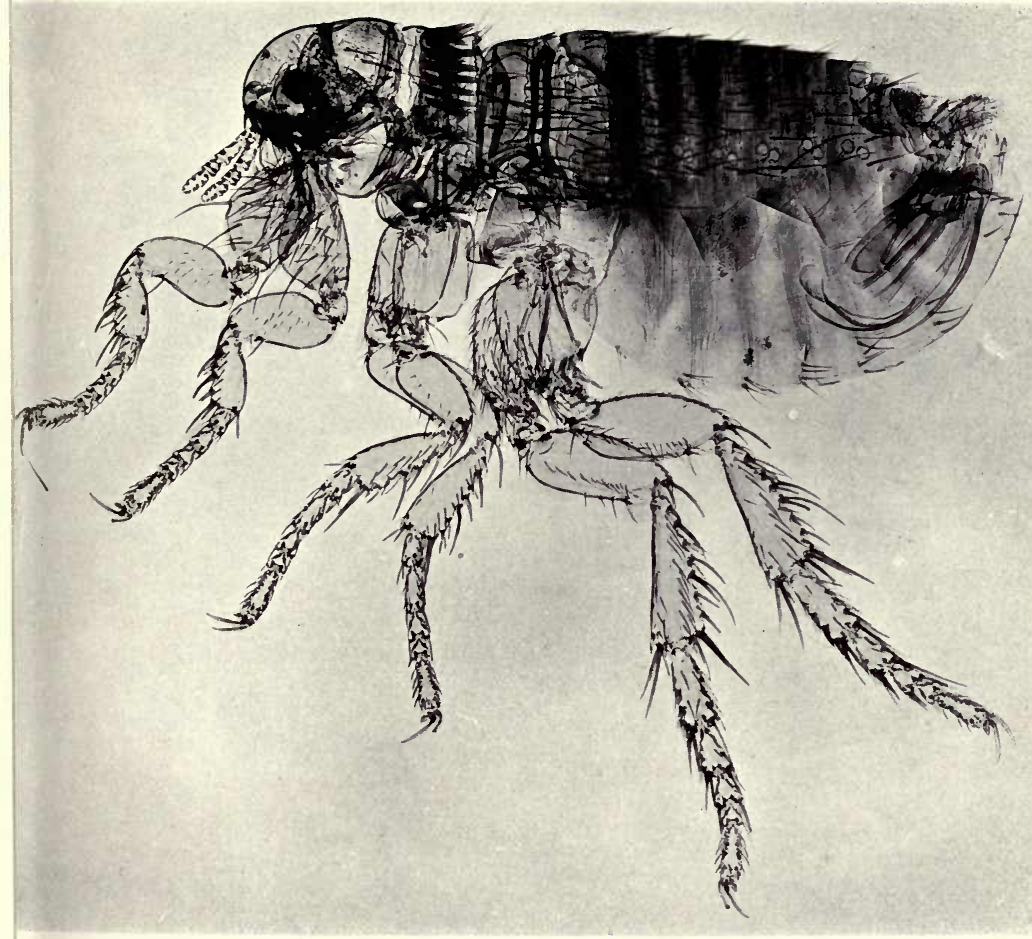



is the most convenient, as exposures can be made by switching it on and off. There are several arrangements of this $k^{*} . d$ on the market, among which Lancaster's Ellipsoid Enlargers may be mentioned; in these the reflectors are of matt opal glass and the lamps are placed at the top of the lantern instead of at the side.

The camera must be clamped to the table or baseboard to give it the necessary rigidity, and its front should be

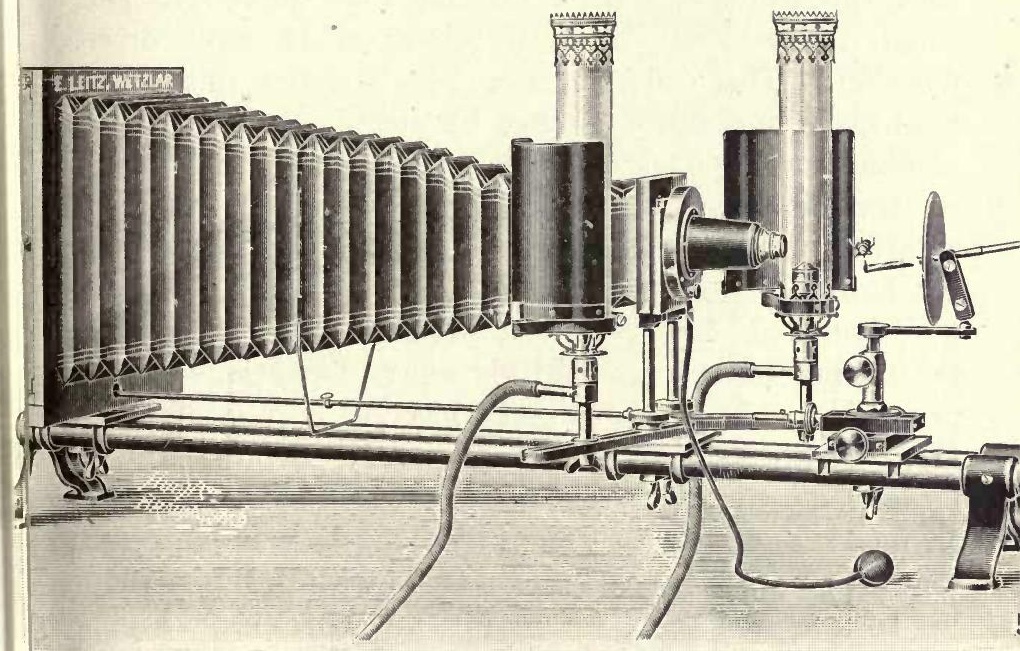

Fig. 41

CAMERA FOI PHOTOGRAPHY OF INSECTS

fitted with a lens board and flange, with the R.M.S. thread, or arranged to carry an adapter into which the small lenses can be screwed. A time and instantaneous shutter is a great convenience when it can be fitted.

A focussing movement at the lens or camera front may be very difficult to manage on account of the length of bellows, and because the lens has to be very often between the lamps or beyond them. These difficulties can be got over in several ways. The bellows can be made 
to draw forward and away from the camera back, and the image then focussed on a piece of paper or card placed over the ground glass, or a rack focussing movement may be fitted to the camera back, or better still to the object-holder.

In the Leitz apparatus arranged for the photography of insects, and shown at Fig. 41, these details are well carried out. The camera slides on tubes in such a way that the front and back can both be moved to get a rough adjustment, and be clamped in any desired position. The object-carrier has a screw motion for final focussing, and is worked by means of a rod from the camera back. A shutter actuated by a bulb is carried on the camera front, to which a conical adapter is attached to bring the small lenses well forward between the lamps.

A vertical camera is sometimes more convenient than a horizontal, particularly when dissections or small preparations in water or spirit have to be photographed. Many of the vertical cameras are very portable, and can be carried into the position where the best light is obtained. Diffused daylight is generally all that is necessary, requiring exposures of a few seconds with ordinary plates, up to about five minutes for autochromes. Fig. 42 illustrates $a$ vertical arrangement, and includes a bull's-eye for projecting a beam of light on to the object.

Lenses. - The lens is the most important part of the equipment. Rapid rectilinear photographic lenses of good make are fairly satisfactory, but the superiority of an anastigmat is very marked. It must be of short focal length, otherwise an impossible bellows extension is required for even small magnifications, and lenses of $4^{\prime \prime}$ to $5^{\prime \prime}$ focus, such as are fitted to quarter-plate cameras, are about the largest that can be conveniently used. The small anastigmat lenses specially designcd for photomicrography are the most suitable. A magnification of 8 
diameters with a $3^{\prime \prime}$ lens, requires a camera extension of $27^{\prime \prime}$, whereas $5^{\prime \prime}$ and $7^{\prime \prime}$ lenses necessitate extensions of $45^{\prime \prime}$ and $63^{\prime \prime}$ respectively to obtain the same magnification.

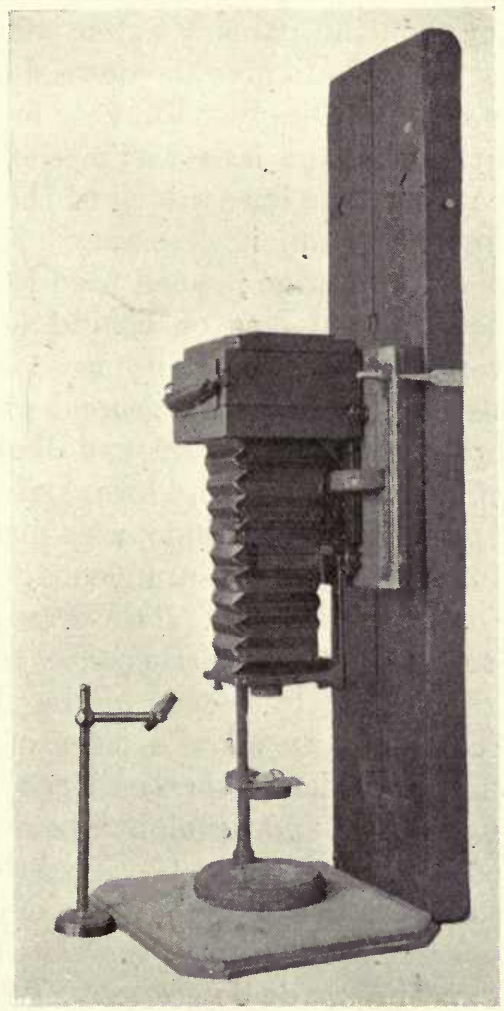

Fig. 42.

VERTICAL CAMERA FOR SOLID OBJECTS

The extension required under any circumstances is calculated from the formula :-

$$
\mathrm{E}=\mathrm{FM}+\mathrm{F}
$$

where $\mathrm{F}=$ focal length of the lens and $\mathrm{M}=$ magnification. 
To Take the Photograph.-Place the object in such a position that it is illuminated to the best advantage, and adjust the lamps, reflectors or condensers until the required amount of shadow is obtained. Refer to the table of enlargements for the camera extension and distance between object and lens to give the desired magnification, adjust the camera accordingly. Roughly focus, and move the object until its image falls within the limits of the plate, as indicated by the lines drawn on the ground glass, altering the magnification if necessary. Colour screens are sometimes of advantage; when used they are placed between the object and lens or behind the lens. The principles governing their use are discussed in Chapter IX. Carefully focus the image on the ground glass, and close the iris diaphragm until the necessary depth of focus is obtained; a lens should be at hand forexamining the image on the ground glass. The dark slide, with plate in position, is then placed in the camera, assurance made that the lens is capped or covered, and the slide drawn, every care being taken to prevent any disturbance of the camera. Exposure is then made by means of the shutter, or by moving the cap, or in its place a piece of card held in front of the lens. The slide is then replaced, and the plate taken to the dark room and developed before the apparatus is disturbed. Time is frequently saved thereby, as any errors in exposure, focus, etc., can be detected during development, or on examination of the plate after fixation, and a second exposure made if necessary. When certainty has been obtained by experience time is saved, however, by making all the exposures necessary one after another, and developing the plates all together in a tank, or two or three at a time in dishes.

The question of exposure is discussed in Chapter $\mathrm{X}$, but the exposures given in the descriptions of the illustrations will serve as a rough guide to the times necessary. 



\section{Photomicrography of Insects.}

Illustrating the use of a blue screen to increase contrast, and the disadvantage of non-colour-sensitive plates.

A Window GNaT $\times 7$

Objective ... ... Ross Planar, 3". F. 4.5.

Condenser ... Lantern condenser and ground glass.

Illuminant ... 30 c.p. electric metal filament lamp.

Screen $\quad \ldots \quad \ldots$ Wratten's H, blue.

Plate $\quad \ldots . .$. Imperial ordinary.

Exposure ... ... $1 \mathrm{~min}$.

Photographed with the apparatus indicated by Fig. 43. The ordinary plate is not capable of showing the detail in the denser brown parts of the thorax; these are, however, readily reproduced on ortho. or panchromatic plates, which should always be used for objects of this sort. The contrasts are increased still more by the blue screen, in this case giving an improvement in the wings and abdomen but not elsewhere.

B LOUSE OF CAT $\times 70$

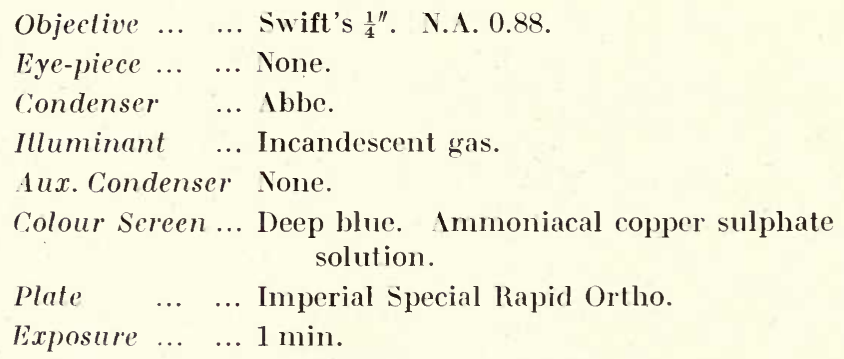

Plate $\quad \ldots$... Imperial Special Rapid Ortho.

Exposure ... $\quad \ldots 1$ min.

The colour of this specimen was very pale yellow, and scarcely differentiated from the balsam in which it was mounted. A deepblue screen was therefore used to give as great contrast as possible, and the iris of the condenser carefully closed down to help in the same direction, but not far enough to produce diffraction effects. 

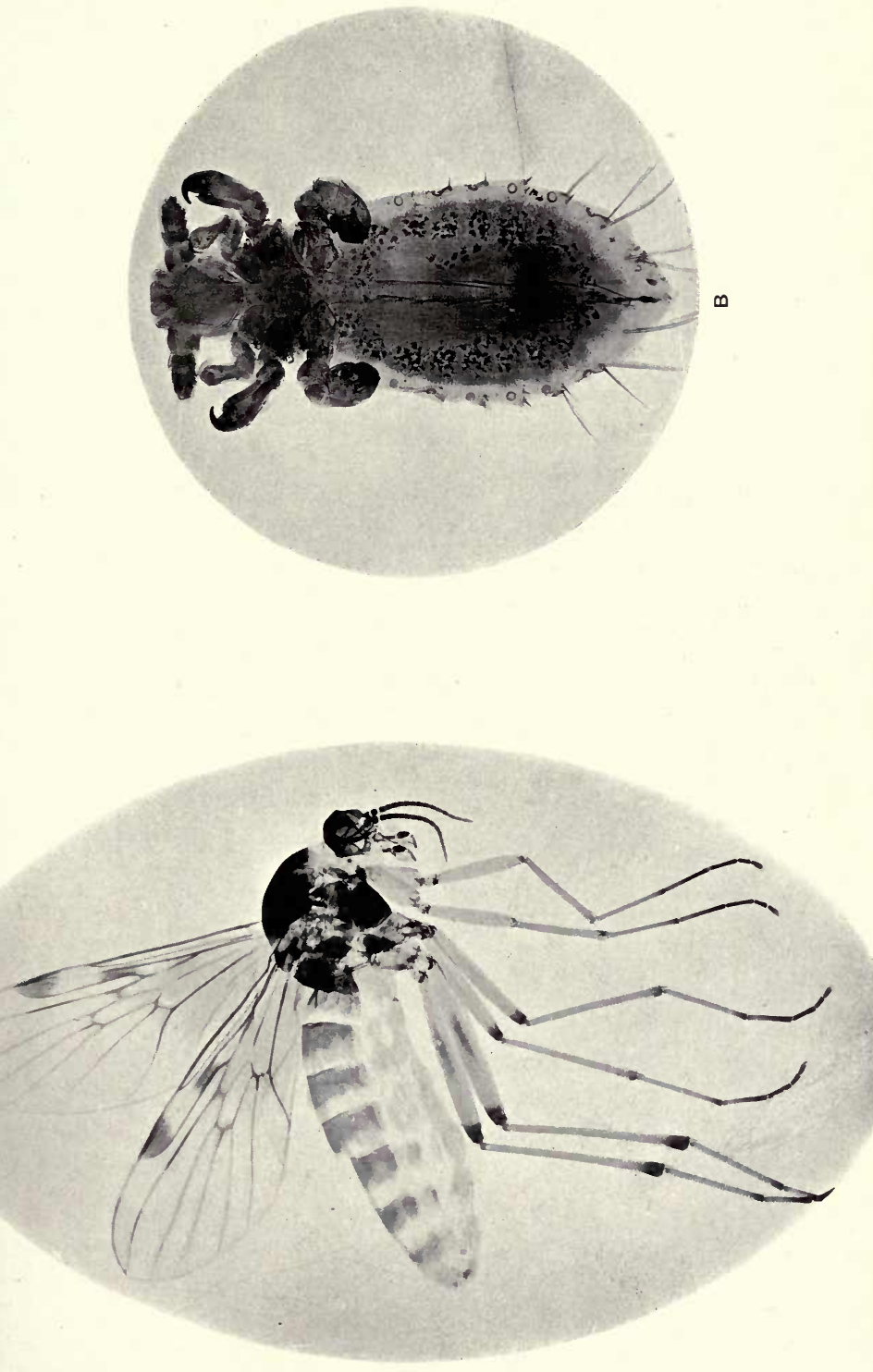



\section{Transparent OBJects}

Illumination by Diffused Light.-Transparent objects may be very evenly illuminated by the simple apparatus described on page 128, and no difficulties arise beyond those already mentioned. Exposures required with two metalfilament lamps of 32 c.p. on a plate whose speed is about 200 H.D. (moderately fast), for an ordinary transparent preparation of an entire insect, are only a few seconds -one to five.

Direct, instead of reflected light, can also be used, if a ground-glass diffusing screen is placed between the lamp and object, but the ground glass causes considerable loss of light, and possibly slight lack of definition in the photograph. Plate 14.A is an example taken in this way.

Illumination by Means of a Condenser.-The above methods of illumination are not sufficiently intense for thick or dark-coloured preparations, and it is best to concentrate the light through the object by means of a condenser. The condensers fitted to projection lanterns are usually quite good enough, and Fig. 43 shows dia-

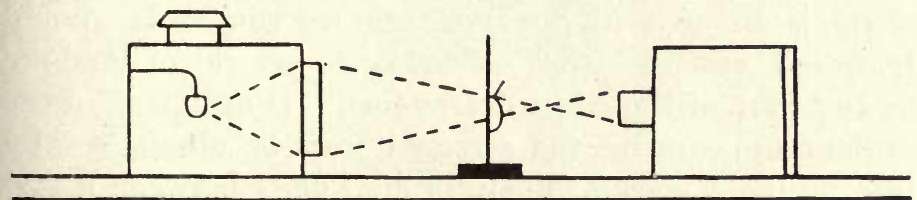

Fig. 43

ILLUMINATION OF TRANSPARENT OBJECTS

grammatically how the apparatus should be set up, and the light managed. The cameras shown at Figs. 1 and 2 can be conveniently arranged for the purpose. An object carrier is made out of a piece of blackened board, in the centre of which a hole $1 \frac{1}{2}$ " in diameter is drilled. Two pieces of clock-spring are screwed to the carrier to hold the object slide across the aperture, and it is attached to a suitable block sliding on the baseboard. 
Approximate positions for the camera and object, and the bellows extension to produce the desired magnification, are found from the enlargement table given on page 276 . A convergent beam of light from the condenser is arranged to cover the object evenly, as shown by the dotted lines in the figure, and fill the aperture of the lens.

Some of the problems connected with illumination, which are among the chief difficulties to be surmounted in photomicrography, are now encountered. There is no question of critical light, but even illumination must be obtained without an image of the radiant appearing on the photograph. At first it is difficult to decide whether the disc of light on the ground glass is even or not, and, if a means for focussing on a card is not provided, it is a good plan to remove the ground-glass focussing screen and place a piece of white card some little distance behind the camera. Inequalities of illumination can as a rule be more easily detected in this way; if shadows appear on any portion of the disc, either the lamp or condenser is moved until the light is perfectly even and white. The defects caused by slight errors of centration of the lamp, or in its distance from the condenser, should be noted, and the steps necessary to get rid of shadows in any part of the circle determined. If an image of the radiant appears on the screen it can be eliminated by interposing a piece of finely ground glass between it and the condenser.

Fig. 44 shows Zeiss' Horizontal and Vertical Camera arranged for the photography of large transparent objects, and a second method of illumination is there indicated, involving the use of two condensers. Those shown in the illustration are two of the Köhler single lens condensers. The collecting lens is No. 1, a large condenser, $8 \mathrm{~cm}$. in diameter and $16 \mathrm{~cm}$. focal length, is suitable for incandescent gas. A somewhat smaller lens $1 a, 6 \mathrm{~cm}$. diameter and $12 \mathrm{~cm}$. focus, is used with smaller radiants, 


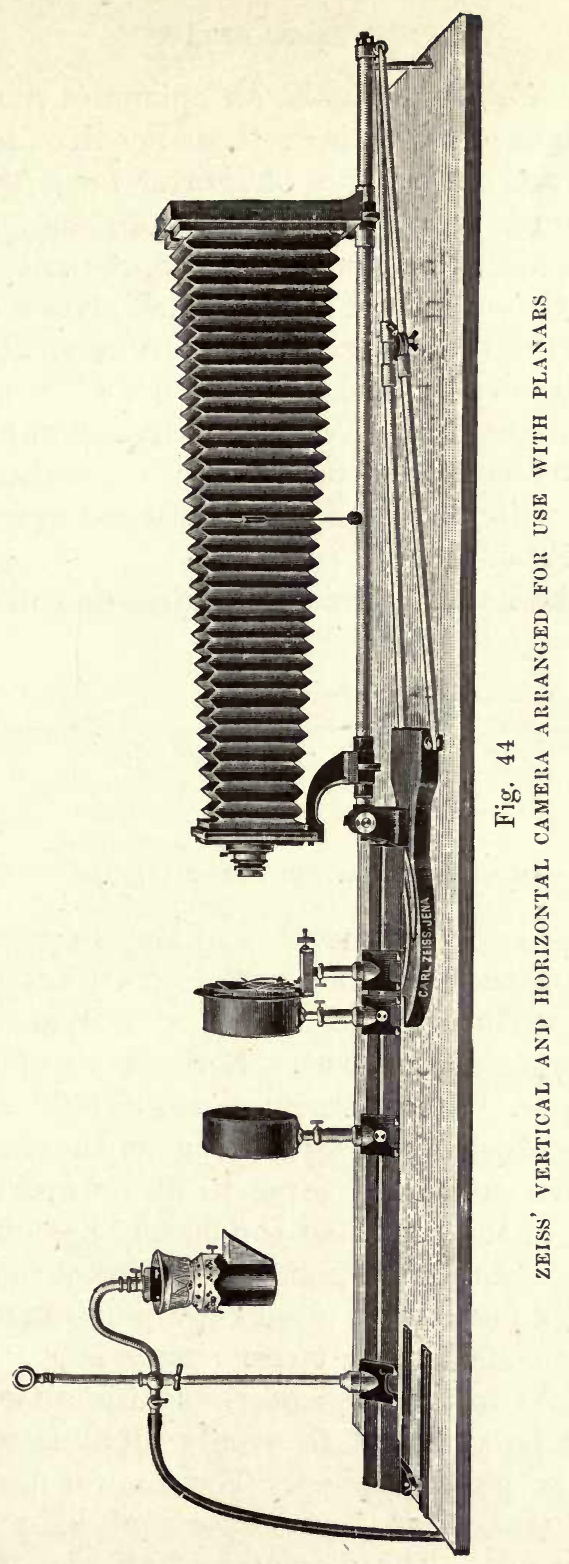


and Messrs. Zeiss now make an aplanatic condenser, 1c, with a N.A. of $\cdot 4$, which is even smaller than $1 a$, and used with small arc lights; it is of shorter focus than 1 or $1 a$, and is placed nearer the lamp than they are. The collector projects an image of the radiant across the surface of the second condenser which gives a convergent beam covering the objective with an even disc of light. The large condenser 3, shown in Fig. 44, is now discontinued and replaced by No. 5, the smallest of the series.

The object is carried on a stage, consisting of an iris diaphragm with clips, and fitted with a screw movement for final focussing.

One of the newer aplanatic condensers can be used in

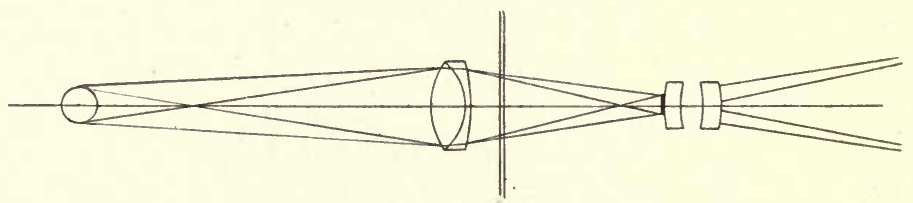

Fig. 45

DIAGRAM OF ILLUMINATION FOR SMALL ANASTIGMATS

place of the two condensers, and the diagram, Fig. 45, shows the method of illumination then adopted. It is drawn approximately to scale for a Watson-Conrady condenser and a $2^{\prime \prime}$ lens, with a Nernst lamp about 5 " from the condenser. A convergent beam of light is projected through the object, forming an image of the radiant across the objective sufficiently large to fill its aperture. The superiority of the corrected condensers has already been referred to, and the fact pointed out that a small one can concentrate a much more intense illumination over a small area than a considerably larger simple lens. The object is placed close to the condenser, and the diameter of the latter must be sufficient to evenly illuminate the whole surface to be photographed. There is no need for it to be greater than that; any excess of light is wasted, or gives trouble. The diameter of an uncorrected con- 



\section{Low-power Photomicrography.}

Median Longitudinal Section of a newly-born Mouse $\times 6 \frac{1}{2}$

Stained in bulk in borax carmine, counterstained in aniline blue and picric acid.

\begin{tabular}{|c|c|}
\hline Objective ... & ... Aldis micro-anasti \\
\hline Ocular. & ... None. \\
\hline Condenser & $\begin{array}{c}\text {... A double convex } \\
7 \mathrm{~cm} \text {. focus pla } \\
\text { microscope sta }\end{array}$ \\
\hline Camera Ext. & $\ldots 25.5^{\prime \prime}$ \\
\hline screen & ... None. \\
\hline Plate & ... Barnet isochromatic \\
\hline Illuminant & ... Petrol vapour. \\
\hline xposure & $\ldots 2$ sec. \\
\hline eveloper & ... Metol-quinol. \\
\hline
\end{tabular}

An example of low-power photomicrography of a large transparent object, involving the use of an objective of great covering power and a condenser of wide diameter. The procedure is described on page 137. 


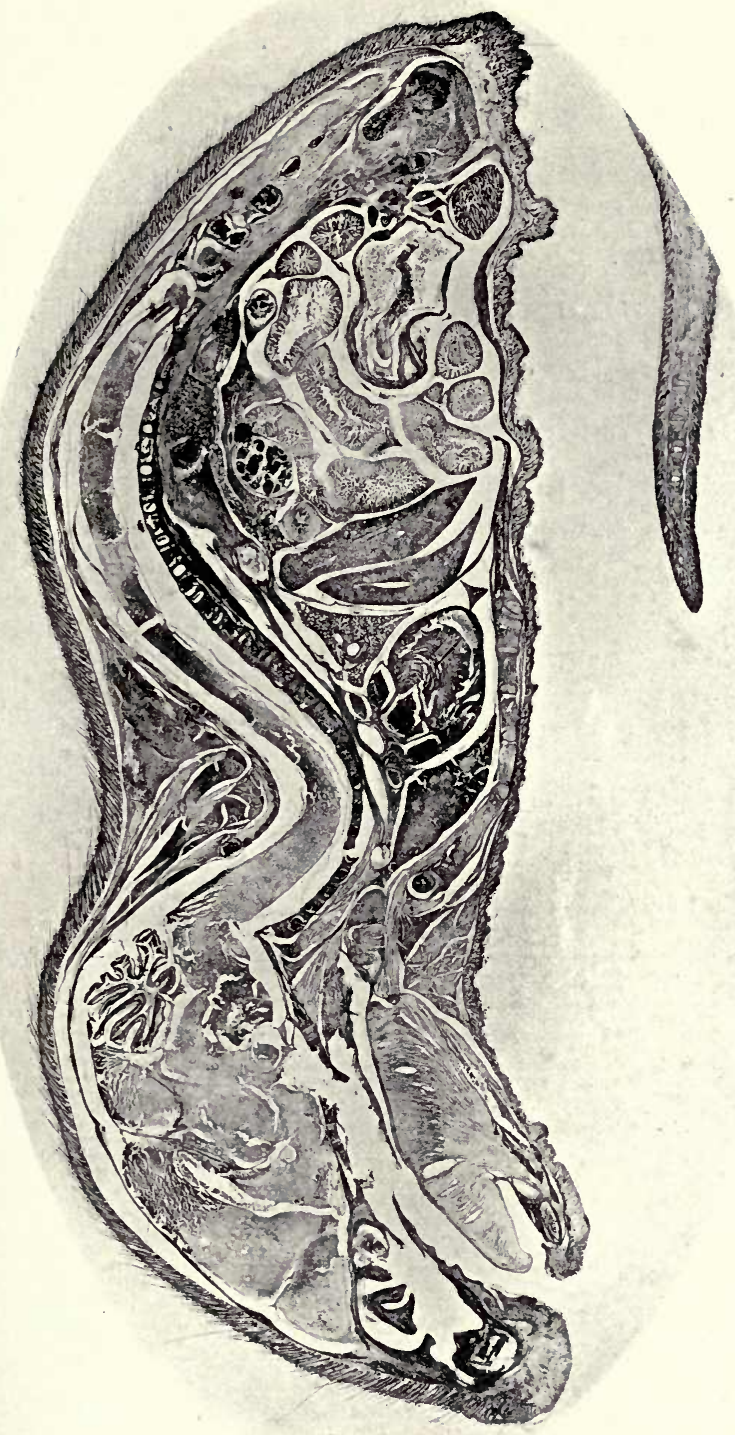



denser must be considerably larger than that of the object, as the light passing through its outer zones must be cut off by a diaphragm ; if utilised, the illumination will not be even, and will fall off at the margin of the field, and show as a distinct circle of under-exposure in the photomicrograph. An apparatus arranged for photography with planars, and using a microscope, is shown in Fig. 46. The substage condenser is seen in use in place of the auxiliary

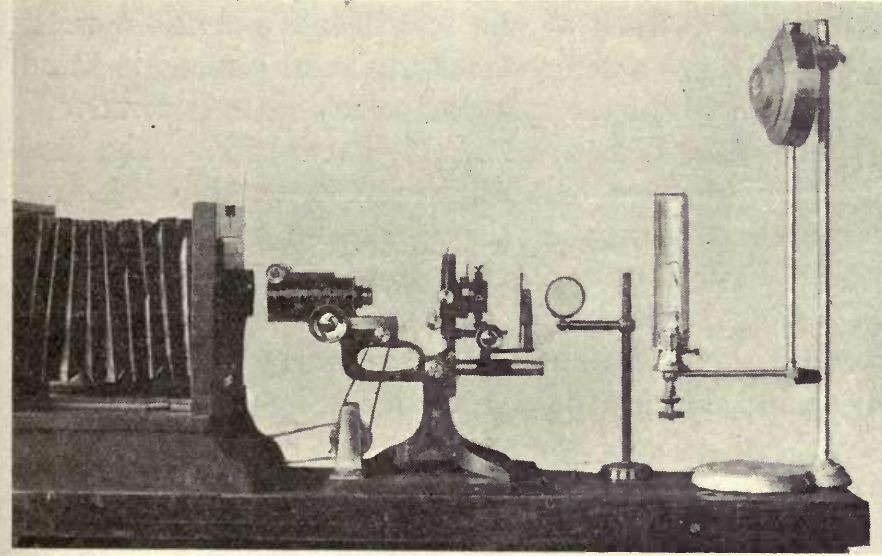

Fig. $46^{\circ}$

PHOTOMICROGRAPIIC APPARATUS ARRANGED FOR USE WITH SMALL ANASTIGMATS

condenser indicated previously. The back combination of an Abbe or achromatic condenser may be of sufficient diameter and focal length to illuminate an object photographed with a $2^{\prime \prime}$ lens, but a $3^{\prime \prime}$ usually requires one of the special condensers mentioned on page 107. A double convex lens of 2 " diameter and 3 " focus, which just fitted the recess beneath the microscope stage, was used to photograph the longitudinal section of the mouse, Plate 15. A Nelson achromatic condenser or an ordinary bull's-eye can also be used. The one chosen is racked up close to the object, or pleced as near to it as the stand will 
permit. There are one or two points to which attention should be directed in this figure. The lamp is a petrol vapour lamp hung on a retort stand, so that its height may be adjusted. A colour screen is attached to the mirror-holder by wire clips, and there is a simple arrangement of pulleys carrying threads by which focussing is carried out. A short dead-black cardboard or metal tube is attached to the camera-front, and makes a light-tight connection by sliding inside the tube of the microscope. The camera is fixed at the front, and extends towards the back, where a screw focussing arrangement is fitted. The draw-tubes of the microscope are entirely removed.

To Adjust the Light and Take the Photograph.-The lamp and condenser are carefully centred to the objective, and the distance between them adjusted, so that an image of the radiant falls across the front of the lens, and fills its aperture. This can most easily be done by holding a card in the position to be occupied by the lens. The camera and microscope are connected light-tight, and the illumination on the ground glass or white card carefully examined, and any unevenness rectified by slight movement of the lamp. The object is then placed in position, roughly focussed, and the bellows extended until the desired magnification is obtained. The stage is rotated if necessary until the object lies in the best position in regard to the plate. The diaphragm attached to the condenser is then restricted until the diameter of the beam of light is just sufficient to cover the object. Excess of light is liable to cause a mistiness over the image. Colour screens, and if necessary ground glass, are put in position. The object is focussed and the iris of the lens carefully closed until the sharpest image is obtained, or to increase depth of focus, and exposure made as previously described. Flare Spot is one of the difficulties encountered when eyepieces are not used, and arises from reflections caused by bright parts in the microscope tube. It appears as a 
black ring in the negative, or white marks in the centre of the print, as in Fig. C, Plate 12. These may be effectually guarded against by covering any bright places with a tube of dead-black paper or velvet.

Advantage of the Wide-Tube Microscopes.-The value of the wide tube adopted almost universally on the better microscopes becomes at once apparent when no eyepiece is used, as the illuminated disc projected by an objective is circumscribed by the tube, and it is frequently impossible to photograph a sufficiently large area of the object with a narrow-tube instrument. The usual diameter of the Continental eyepiece mount is $23 \mathrm{~mm}$. and the tube into which the eyepiece slips, or a diaphragm within it, limits the pencil of rays from the objective, and consequently the area of the object included in the photomicrograph when no eyepiece is used. To increase the field the draw-tubes are removed from the microscope, and the diameter of the projected disc is then limited by the outer tube, which varies from $30 \mathrm{~mm}$. on small stands to $42 \mathrm{~mm}$. and even $50 \mathrm{~mm}$. on the large ones. Most objectives will not project a sharp image of their entire field of view, and the area bounded by the widest tube is often greater than they can satisfactorily cover. But even so, the field is unnecessarily circumscribed by the narrow tube; and to make proper use of the small photographic lenses, which are constructed with a view to large covering power, and have a very large flat field, even the wide tubes are none too large.

Dissecting microscopes are made without tubes, and can be conveniently used with a vertical camera when it is desired to obtain the largest possible field and at the same time make use of a microscope stand to earry the object and lens. Some old-fashioned microscopes from which the tube is removable are also suitable, and can be used in a horizontal position as well.

Telephoto Lenses.-Telephoto lenses can be used to 
obtain magnified images of small objects with very considerable success, and have the advantage over microanastigmats of a much longer distance between subject and lens, combined with a shorter camera extension to give the same magnification. There is, however, the disadvantage that the area of good definition is not very large, but their special properties make them very suitable for natural history photography, in which a large field is not of the greatest moment.

An ordinary anastigmat lens can be made into a telephoto combination by the addition of a negative element of shorter focal length, and many makers supply a suitable negative for use with the photographic lenses. Any defects of the positive lens are magnified if this course is taken, and it is better to use a specially made combination in which the elements have been carefully adjusted the one to the other.

The magnification obtained depends on the focal length of the combination, on the relative object and image distances, and on the separation of the lens components.

The focal lengths of the components of the "Adon," a typical telephoto lens, are, positive $4 \frac{1}{2}$ ", negative $2^{\prime \prime}$. The separation of the two lenses is adjustable by rack and pinion up to $2 \frac{1}{2}$ ". For photomicrography a larger separation than this is needed, and extra tubes are supplied for the purpose. The following figures give the extensions, etc., required by this lens, compared to a $3^{\prime \prime}$ anastigmat.

\begin{tabular}{|c|c|c|c|}
\hline Magnification. & $\begin{array}{l}\text { Ol,ject } \\
\text { Distance. }\end{array}$ & $\begin{array}{c}\text { Camera } \\
\text { Extension. }\end{array}$ & $\begin{array}{l}\text { Extension for } \\
3^{\prime \prime} \text { lens. }\end{array}$ \\
\hline 3 & 14 inches & 10 inches & 12 inches \\
\hline 6 & 10 & 11 & 21 \\
\hline 6 & 14 & 22 & 21 \\
\hline 12 & 7, & 10 & 39 \\
\hline
\end{tabular}


The increased simplicity in illumination obtained by the longer object distance is obvious ; and in the case of larger magnifications the difference in camera extension is very considerable.

The lens separation required in the above cases varies from about $5^{\prime \prime}$ to $11^{\prime \prime}$; a small change in separation is equivalent in its effect on magnification to a rather large change in camera extension, and in consequence it is simpler to obtain final focus by adjustment of the lens after the camera extension and magnification have been roughly fixed. 


\section{CHAPTER VIII}

\section{CRITICAL PHOTOMICROGRAPHY}

In this chapter the methods of Critical illumination, as defined by Mr. Nelson, and the modifications that have been mentioned as suitable, are applied to photomicrography, and details are given of the adjustments and processes adopted to take both low- and high-power photomicrographs under those conditions.

The experiments detailed in Chapter VI supply all the data required for obtaining the best illumination possible, critical light, and an even field. The following rules are based upon them, and should always be followed for medium-and high-power objectives used either with or without eyepieces. Low-power objectives are also best treated in the same way provided suitable condensers are available, but in most cases it is necessary with objectives of $2^{\prime \prime}$ and lower power to make use of the methods described in the last chapter to get an evenly illuminated field.

The number of operations that must be carried out before the whole apparatus is in adjustment for critical light may seem unnecessarily tedious for simple work which does not really require such care, but, if the baseboard or optical bench has been properly constructed, the microscope, lamp, condensers, and camera will be found to be almost exactly centred. Only very small final adjustments should be required, and these are so quickly effected that there is no need to omit them at any time. 
Vertical Cameras.-The instructions given in this chapter and the accompanying illustrations refer particularly to horizontal cameras, but they apply equally well to vertical cameras, and can in some respects be carried out more easily under those conditions. The adjustments are just the same, except that the direction of the beam of light is changed by the mirror. The microscope is placed under the camera, and centred by means of the disc of light projected on the ground glass. Centration of the light is effected very easily by movement of the mirror, while the condensers, light-filters, cooling trough, etc., are disposed exactly as for a horizontal camera. If the mirror can be arranged on the level of the condensers and lamp, their optical axis is kept horizontal, otherwise the condensers are tilted down, as shown in Fig. 7. Light-tight connection between the camera and microscope is made either by the double cells or velvet sleeve (Figs. 4 and 71).

Preliminary Examination of the Object.-Before proceeding to take a photomicrograph it is necessary to make oneself familiar with the object and its characteristics, and decide what details, if any, are to be particularly brought out, and under what magnification or method of illumination it is best displayed. The examination is usually carried out in the following way, after a careful centration of the condenser, etc.

1. Search the whole preparation with a low-power, and decide whether the photomicrograph is to include the whole or only part of the object, and find the positions that best display the particular points of importance.

2. Locate the desired points, if necessary, by means of the verniers fitted to the mechanical stage, so that they may be rapidly found when using a high-power at any future time.

3. Examine the object with various combinations of objective and ocular, and decide which combination is 
best. A camera extension of $10^{\prime \prime}$ will give a photomicrograph of similar amplification to that observed directly in the microscope. Variations in camera length give proportional variations in magnification.

4. Examine the effect produced by closing the iris diaphragm, and decide on the best aperture to employ; it should be kept as large as possible, as diffracion effects which are easily overlooked become distinct in a photomicrograph.

5. Place various colour screens in the substage ring or between the lamp and mirror, and select the most suitable, as explained in Chapter IX.

6. Try the effect of dark ground and oblique illumination (Chapter XI), but use the latter with great caution as it may produce false images.

Besides this careful examination of the specimen there are various optical details that are best attended to before the microscope is transferred to the photomicrographic stand. Of these a correct centration of condenser and objective has already been mentioned. For high-powers it is necessary to consider the thickness of the cover-glass, and make any adjustment of the correction collar or tube length that may be found desirable, as explained on page 69 . And above all, the lenses and slide must be examined for any specks of dirt, and, if found, their location on objective, eyepiece, or condenser may be decided by turning each of these round in turn and noticing whether the specks move. The dirt is then removed by a silk handkerchief or soft tissue-paper.

Having decided whether the object is to be photographed as a whole or in part, and what magnification and method of illumination best display the necessary detail, attention must be directed to the camera and optical bench, and such dispositions made as will best attain the desired results. Systematic instructions are given for obtaining critical illumination and an even 



\section{Photomicrography of Geological preparations.}

A Section of Pitchstone $\times 60$

Objective ... ... Leitz No. 3 Achromat. $16 \mathrm{~mm}$. N.A. 0.3.

Ocular $\quad \ldots \quad \ldots$ Leitz 0 .

Condenser ... Watson's Universal Achromatic. Stopped down.

Illuminant $\quad \ldots$ Liliput 4 amp. arc.

Aux. Condenser Double convex lens, giving parallel beam.

Screen $\quad \ldots \quad \ldots$ Wratten's B (green) and E (orange).

Plate $\quad \ldots \quad \ldots$ Wellington Anti-Screen.

Exposure ... ... $1 \mathrm{~min}$.

The section of Pitchstone was rather thick, and in order to get depth of focus the substage iris was closed down to about a quarter of its full aperture. This, together with the use of the two screens $\mathrm{B}$ and $\mathrm{E}$, necessitated a comparatively long exposure.

B Transverse Section of Fossil Wood (Lepidodendron selaginoides) $\times 13$, from Halifax.

Objective ... .... Seibert's 0 Achromat. $36 \mathrm{~mm}$.

Ocular .... ... None.

Condenser ... Abbe with front lens removed.

Illuminant ... Liliput 4 amp, arc.

Aux. Condenser Double convex lens, giving parallel beam.

Screen _...... Green glass.

Plate $\quad \ldots$.... Ilford Chromatic.

Exposure ... ... 4 sec.

Developer ... Pyro-formosulphite. 


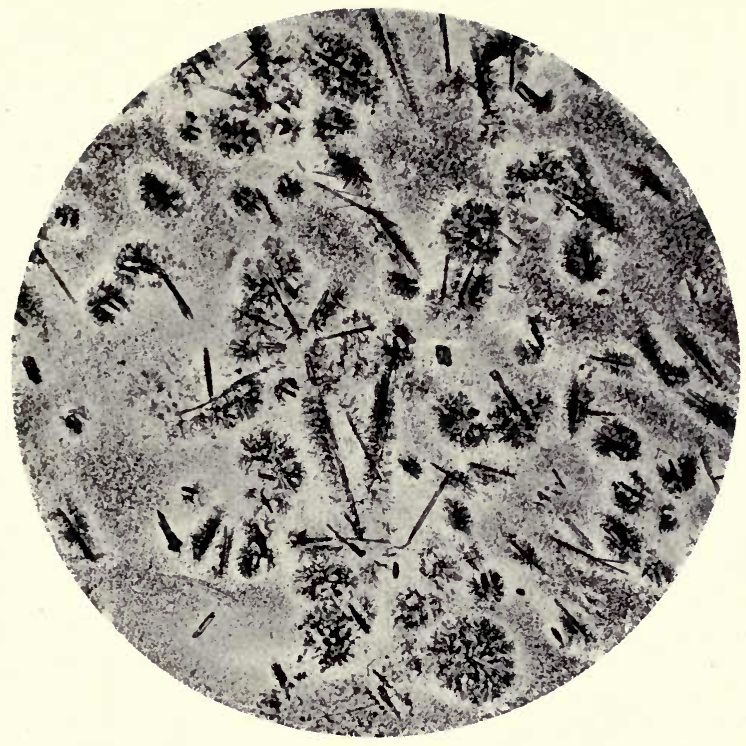

A

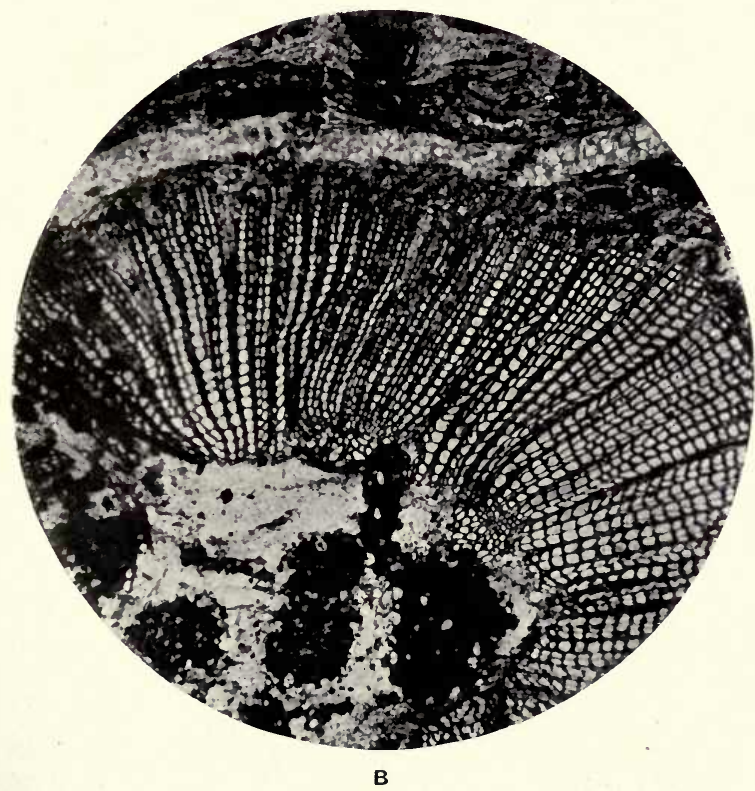



field without an auxiliary condenser, followed by a description of the adjustments necessary when an auxiliary condenser is used to give a parallel or convergent beam. Under proper conditions any of these methods of illumination can be used, the choice often depending on the apparatus available. The principles underlying them have already been explained, and the processes themselves described, so that it is only necessary to give the complete scheme in outline.

The microscope is placed on the optical bench, and connected with the camera. The lamp is placed as accurately as possible in the optical axis. A white card used in one of the ways already described will be found preferable to the ground glass for adjustment of the illumination, and the camera may if preferred be removed while the adjustments 4 to 6 are carried out.

\section{ILLUMINATION WITHOUT AN AUXILIARY CONDENSER}

1. Centre the Microscope.-Fit a low-power objective, but no eyepiece, and see that the disc of light appears in the centre of the focussing screen.

2. To Centre the Light.-Remove the objective, condenser, and focussing screen, look through the back of the camera down the microscope tube. Move the illuminant until it appears in the centre of the tube. With vertical camera move the mirror until the light appears central.

3. Centre the Substage Condenser.-Screw a low-power objective on to the microscope, place in a low eyepiece, or dispense with an eyepiece altogether; close the iris diaphragm to a pin-point and focus its small aperture on the ground glass by racking the microscope tube up or down. If the image of the diaphragm is not central, adjust the centring screws until this is so.

4. Adjust Magnification.-Place the object on the stage, attach the objective and ocular it is decided to use, 
and extend the camera or move the card so as to obtain the desired magnification, or to make the image fall within the limits of the plate.

Adapt the substage condenser to the objective in use by removing the front lens if necessary.

5. Obtain Critical Illumination.-Open the iris and focus the object on the ground glass with a low or medium objective. Now rack or slide the condenser (with its front lens removed, if that is not to be used) down or up until the image of the illuminant appears in focus at the same time and across that of the object. If the image of the flame is not quite central move the lamp or mirror until centrality is obtained ; do not alter the adjustment of the condenser.

6. To obtain Even Illumination.-Focus the object on the screen. Move the slide on the stage till a clear space is in front of the objective, and very carefully examine the disc on the ground-glass or white screen, and see that the illumination is perfectly even.

If a change of objective has been made it may be necessary to slightly alter the centring of the condenser, but the movement should be at the most very slight. If the image of the radiant does not cover the field, slightly rack up the condenser till the illumination is even. Remove the ocular and examine the back lens of the objective; if not full of light it is better to use an auxiliary condenser as described next.

7. Adjust the Substage Iris.-Carefully examine the image on the ground glass while gradually closing the iris diaphragm. Use the aperture which gives the best result in regard to clearness of image, and sufficient depth of focus without any signs of diffraction. Examine the back lens of the objective to find what portion of its aperture is utilised.

8. Focus.-Bring the image of the object into position over the marked area on the ground glass corresponding 
to the plate to be used. Carefully examine the image for specks of dust on lenses or slide, and to see that the condenser aperture is correct. Place the colour screen in its position and carefully focus ; if apochromatic lenses are used focus can be found in white light and then the screen adjusted, but this must not be done with achromatic objectives. It is as well to focus finally on the clear glass, for which purpose a focussing lens must be used, and held in such a position that the fine cross marked with a diamond on the inside of the screen is in focus. As the image is liable to go out of focus on account of vibrations or the heat of the lamp, it should be examined again before inserting the dark slide if there is any delay before exposure.

9. Exposure.-Place the dark slide in the camera without shaking the latter, place a piece of card between the microscope and lamp, draw the slide and move the card aside for the time of exposure. Replace the card and slide. During the exposure allow as little extraneous light as possible to fall on the lens or object.

\section{ILLUMINATION WITH AUXILIARY CONDENSERS}

Parallel Light.-Operations 1 to 5 are carried out as already described.

6a. Adjustment of Paralleliser.-The auxiliary condenser or paralleliser is placed with its plane or concave side towards the lamp, and between it and the microscope, with the radiant at its principal focus. It is correctly placed when it appears quite evenly full of light, and projects a perfectly even disc on to the substage condenser. To determine whether the beam is parallel blow tobacco smoke across it, or move a white card backwards and forwards between the paralleliser and microscope, also examine the disc projected on the card for any unevenness. 
7a. Refocus Substage Condenser for Parallel Light.-This is done by shutting down the iris of the auxiliary condenser, if it has one, or by covering it with an opaque disc having a small central aperture, or by holding a pencilpoint in front of it. Focus the image of either of these on the ground glass by racking the substage condenser up or down.

8a. Centre the Auxiliary Condenser.-Centre the image of its iris by means of the centring screws attached, or move the condenser and lamp together until the image of the iris in the object plane is centred. A good method of examining the collimation of the whole apparatus is afforded by a cap with a very small central aperture fitting the microscope in place of the eyepiece. When the substage iris and auxiliary iris are closed to their smallest apertures light should pass uninterruptedly through the three pin-holes, but any error in centration will prevent that.

Auxiliary Iris.-It is of the greatest importance that the entire aperture of the substage condenser be covered with an even white disc of light if its full aperture is to be used. To make sure that this is so, a card is held in front of the substage condenser : a shadow in the centre of the disc shows that the lamp and condenser require adjustment. Any colour fringes must lie entirely beyond the aperture and should be cut off by means of the auxiliary diaphragm.

Cooling trough and colour screens are then placed between the condenser and microscope, and focussing and adjustment of the substage iris are carefully carried out as before, and the exposure made.

A more intense illumination, but still furnished by a parallel beam of light, may be obtained in the way shown in Fig. 47, which is a diagram of Beck's Optical Bench and Imperial Microscope. An image of the radiant in the lantern is projected on the bench diaphragm placed at the focus of a small aplanatic condenser, which directs 
the rays in a parallel beam on to the substage condenser. The lantern condenser is partially corrected.

The adjustments are substantially the same as are required when the radiant, instead of its image, is at the focus of the paralleliser.

The Cooling Trough.-Whenever rays from an intense source of light are concentrated on to the microscope, the

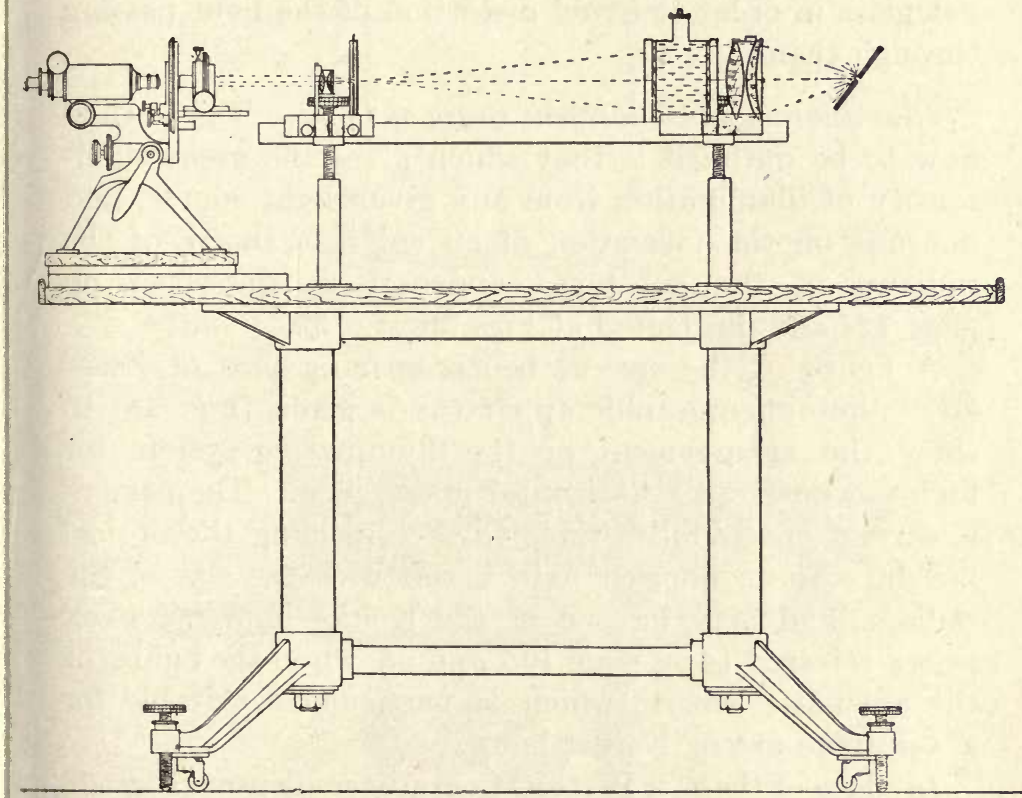

Fig. 47

ILLUMINATION BY A PARALLEL BEAM OF LIGHT

necessity arises for precautions to avoid damage to lenses and preparation by the heat of the radiant, and a water-trough must be inserted as indicated in Fig. 47. Water is the only liquid required, but the trough must be of ample thickness: $3^{\prime \prime}$ or $4^{\prime \prime}$ is none too much for a 30 -ampère arc lamp; a 5-ampère lamp will require a thickness of about 1 ". With less intense light sources there is no neces- 
sity to introduce a cooling trough if care is taken to keep the light on no longer than is necessary, but its use is to be recommended whenever valuable preparations are on the microscope. The construction of small troughs is described in the section devoted to light filters, for which purpose they are most frequently used. The large ones illustrated here and at Fig. 48 are made of good flat glass in order to avoid distortion of the light passing through them.

Illumination by Convergent Beam of Light.- The method now to be outlined is that which gives the greatest intensity of illumination from any given light source, and depends on the formation of an enlarged image of the radiant on the substage condenser as described on page 124 and illustrated at Figs. 38 and 39.

A figure of the optical bench forming part of Zeiss' large photomicrographic apparatus is given (Fig. 48) to show the arrangement of the illuminating system for high-powers with a horizontal microscope. The camera is carried on a similar table to that forming the optical bench. The condenser used varies with the size of the radiant, and may be one of the Köhler double convex lenses referred to on page 105, and shown in the figure, or the aplanatic lens $1 c$ which is particularly suitable for a 4-ampère arc or Nernst lamp.

In place of these, a Watson-Conrady condenser (Figs. 38 and 39), a Nelson, or other aplanatic condeniser, or a simple double convex lens such as is shown at Fig. 7 may be used.

The microscope and lamp are arranged for critical light as described above (operations 1 to 5 ).

$6 b$. The condenser is then placed on the optical bench in such a position that it projects an image of the radiant on the substage diaphragm. Small aplanatic condensers require to be placed much closer to the radiant (about $1 \frac{1}{2}$ ) than the Köhler lens figured. 

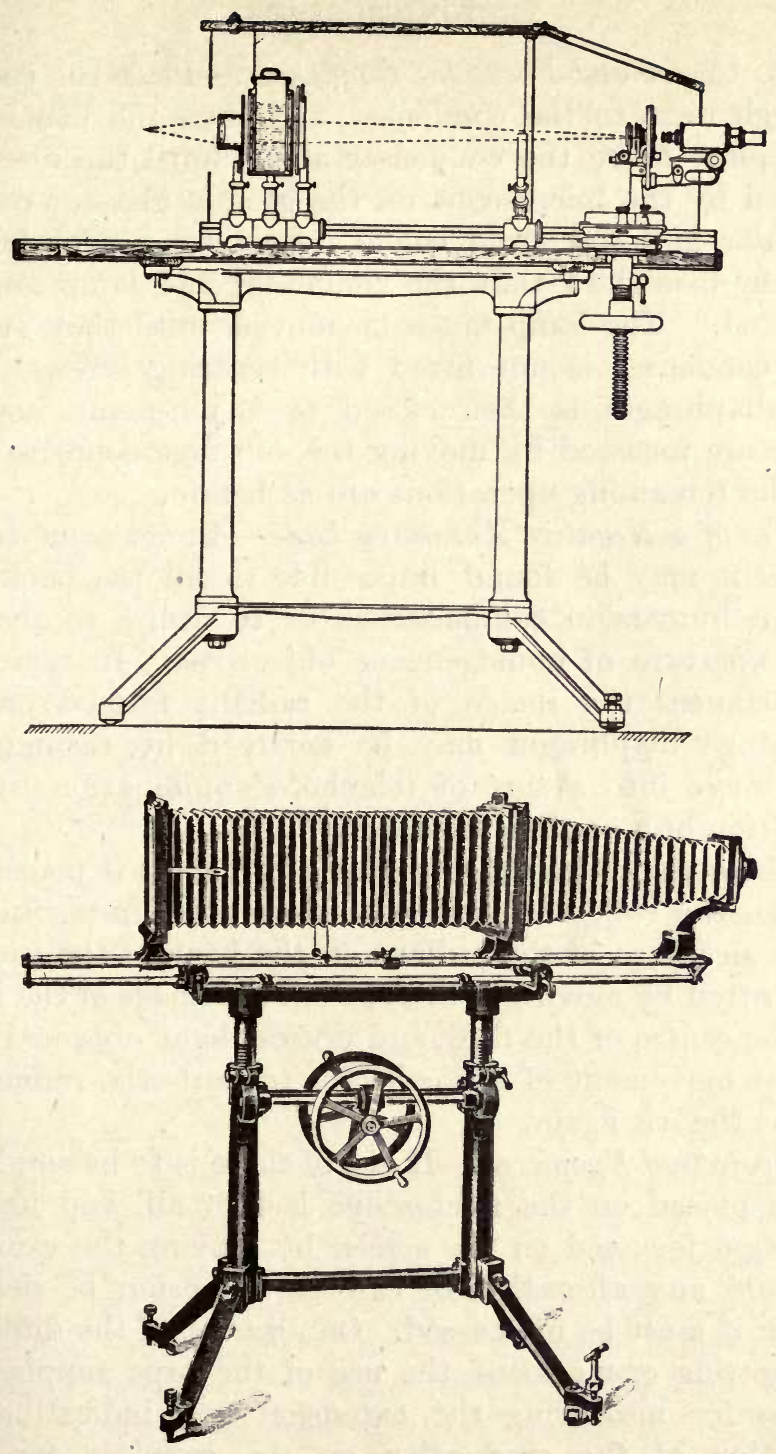

Fig. 48

ZEISS' LARGE CAMERA AND OPTICAL BENCII 
7b. Centre and Focus the Condenser.-Place the cooling trough next to the condenser, and then the bench diaphragm. Move the condenser about until the disc projected by the microscope on the ground glass or card is circular and free from colour. Spots or colour fringes on the disc show that the condenser and lamp are not centred. The lamp must be moved until they are, as the condenser is not fitted with centring screws. The iris diaphragm is then closed to a pin-point, and its aperture focussed by moving the substage condenser.

The remaining operations are as before.

Use of a Negative Auxiliary Lens.-Under some conditions it may be found impossible to fill the back lens of an immersion condenser so as to utilise to the full the aperture of homogeneous objectives. In these circumstances the image of the radiant formed on the substage diaphragm may be enlarged by turning the bull's-eye into a sort of telephoto combination by the addition of a concave or negative lens.

The concave lens (about 6 or 7 in. focus) is placed between the collecting lens and microscope, in position to give an image of the radiant on the back of the iris. It is centred by moving it about until the image of the iris is in the centre of the field, and critical light obtained by a slight movement of the collector towards the radiant to focus the iris again.

Projection Eyepieces.-If one of these is to be employed it is placed on the microscope last of all, and its diaphragm focussed on the screen by moving the eye-lens. Should any alteration of camera extension be decided upon it must be refocussed. On account of the difficulty of getting exact focus, the use of the tape supplied by Zeiss for measuring the extension and indicating the position of the graduation on the eyepiece for that extension is to be recommended. 


\section{Colour Screens for control of contrast and improvement of definition.}

A, B, and C stem of Clematis vitalba. Transverse Section $\times 28$

stained by immersion in picro-aniline blue. The lignified cell walls are colomed yellow (Y), and the collulose walls blue (B).

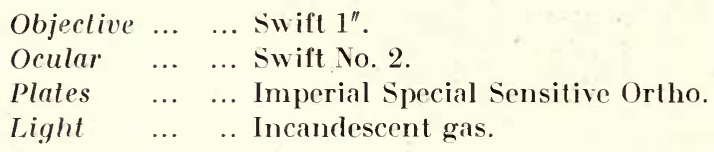

A No screen. Exposure 1 second. The yellow appears darker than the blue.

B Wratten's C (blue-violet) screen. Exposure 10 seconds. Screen nearly complementary to the yellow stain. The contrasts are increased, the yellow becomes darker and the blue lighter.

C Wratten's B and E screens, giving monochromatic yellowgreen light of wave length 5600-6000 Exposure 10 minutes.

This figure gives a fairly correct representation of the visual appearance of the section, the blue being darker than the yellow, with a notable improvement in definition and detail, due to the use of monochromatic light of wave length suited to the corrections of the objective.

D Leaf of Cycas revoluta. Transverse section $\times 25$, slained red and blue.

Objeclive ... ... Leitz No. 3 Achromat.

Ocular ...' ... Leitz No. 2.

Condenser ... Abbe.

Illuminanl ... Incandescent gas.

Screen $\quad \ldots \quad \ldots$ 1. Left side, without screen.

2. Right side, yellow screen (5 times).

Plate ....... Imperial Special Rapid Ortho.

Exposures $\quad$... 2 sec. and $10 \mathrm{sec}$.

The section is stained mainly blue. The illustration shows the advantage of a yellow screen in giving the blue portions their correct depth of tone, and due contrast.

These four photomicrographs all taken on Orthochromatic plates. 



\section{CHAPTER IX}

COLOUR SCREENS AND COLOUR SENSITIVE PLATES

Light Filters or Colour Screens.-It has been already pointed out that the complexity of white light and the different refrangibilities of its component rays lead to defects in the image formed by achromatic objectives, and attempts to take photomicrographs with ordinary white light will soon convince the worker that, in many cases, other defects occur which cannot be referred to these causes. Besides a possible lack of sharpness, due to the imperfect corrections of the lenses, there may be a lack of contrast or of differentiation in the parts of a preparation, or of an object from the field in which it lies, and no care in the adjustment of the illuminating system or in the use of the most perfectly corrected lenses will suffice to give a perfect photomicrograph. The insertion of a piece of coloured glass in the system between the radiant and condenser very frequently produces a vast difference in the resulting negative. The glass acts as a "Light filter" or "Colour screen," and only allows a certain portion of the rays emitted by the radiant to pass through it. The photomicrograph is therefore taken with light of a selected colour onlygreen, for example - and becomes sharp, instead of slightly out of focus in appearance, on account of the improved performance of the objective under these conditions. Better contrast and increased resolution may also be apparent in the negative, and in fact properly selected colour screens are practically indispensable in photo- 
micrography, and it is essential that their action should be clearly understood in order that the best selection should be made for whatever object is under examination. Absorption Spectra-Monochromatic Light.-The spectra of most radiants consist of a continuous band of colours - violet, blue, green, yellow, orange, and redgrading the one into the other, but if the light is passed through a piece of coloured glass or through a coloured liquid, and then examined by means of a spectroscope, the spectra are found to have quite a different appearance. They are no longer continuous, but broken here and there by dark spaces or "Absorption bands." The bands of colour still remaining depend on the nature of the screen, and may be narrow and almost pure in colour, or broader and show several colours; they sometimes end quite sharply on one or both sides, but in other cases gradually fade away. These bands of colour constitute the "Transmission bands" of the filter in question, and the spectrum made up of coloured bands and dark spaces is called the "Absorption spectrum" of the dye or colouring matter ; if it consists of a single narrow coloured band in any region, the light transmitted by the screen is "Monochromatic." Strictly speaking, this term should refer to light of one wave length, giving a spectrum consisting of one line only, but this is not often obtained in practice, except when the light from an incandescent vapour is used, as in the Mercury Vapour lamp.

The Efficiency of Light Filters depends upon the particular rays of light that they respectively transmit or absorb, upon the sharpness of the transmission band or bands, and on the amount of light transmitted. In regard to the last point a screen giving monochromatic light, with very sharp demarcations between the transmission band and the regions of absorption on either side, would be of very little use if there was much loss of 
intensity in the transmitted part. There is always some absorption of light in the transmission bands, and the aim of the screen-maker is to make that loss as small as possible, consistent with a sharply defined band and total absorption in the other parts of the spectrum. The majority of filters do not transmit monochromatic light, but a band extending over a considerable length of the spectrum, or several such bands, and it is impossible to tell merely by inspection whether a screen is monochromatic, but the required information is very readily obtained by examining the transmitted light by means of a direct-vision spectroscope.

Material of Screens. - Screens may be made of coloured glass, dyed gelatine either unmounted or mounted on glass, or of solutions contained in glass cells. Of these ordinary glass is liable to refract the light that passes through it more or less unevenly, and on that account its use is to be deprecated for critical work, as it is no good taking every care that lenses and condensers should be free from aberrations, and then introducing these with the screens. For low- and medium-power and even highpower work, when high resolution is not required, glass may be used without fear, if it is of reasonably good quality and parallel-sided. The same remarks apply to the use of ordinary glass for making the troughs for liquids.

Glass flats are now made in various colours, and, as their surfaces are as carefully worked as those of a lens, they do not introduce these errors, and form the best material for colour screens and the sides of glass vessels ; but unfortunately their cost is in many cases prohibitive, and it is difficult to get a series with the desired absorptions. Dyed gelatine forms an excellent substitute for glass, so far as efficiency and freedom from errors are concerned, but with the disadvantage that screens made of it are very liable to injury. Gelatine, however, has the 
advantage that it may be easily dyed to any desired colour in order to produce a series of screens of definite transmissions.

Sets of Screens.-Messrs. Sanger-Shepherd and Messrs. Wratten and Wainwright make screens of dyed gelatine film for photomicrography whose absorption spectra are given, and such a series puts light control on a definite basis and cannot be too strongly recommended. The makers provide a list of the wave lengths transmitted by all of them separately, and by pairs which together transmit approximately monochromatic bands. It is best to have these screens mounted between optical flats, as the film is very easily scratched; to cement them between ordinary pieces of glass may do well enough for low powers, but the liability to the introduction of errors is then even greater than with ordinary glass screens. They are obtainable in $1^{\prime \prime}$ and $2^{\prime \prime}$ squares.

To Make Screens of Dyed Gelatine.-It is possible to make screens of this type by clearing old lantern slides in Farmer's Reducer and dyeing the film to the desired colour. To preserve the screen a cover-glass is bound over it, as with lantern slides, or two may be bound together face to face to increase the absorption. Better screens can be made by coating thin optical flats with gelatine and then dyeing them, rather than by making use of cleared photographic plates or lantern slides.

The solution of gelatine is made by soaking one part of best gelatine in ten to thirteen parts of water until well softened; complete solution is obtained by placing the containing vessel on a water bath, or in a shallow saucepan of boiling water. The solution is filtered whilst hot through glass wool and a little poured on the clean glass. By carefully tilting first to one corner and then to the others an even coating is easily obtained, and any superfluous gelatine run back into the bottle. When the film has set, it is dyed by immersion in the dilute dye 
solution, 1 part dye in 1000 parts water, until it is judged to be dark enough, then rinsed in water and left to dry.

The dyes employed for microscopical work, picric acid, etc., can be used for dyeing the gelatine. The absorption bands of a few suitable dyes are included in the diagram of absorption spectra, page 159, but the screens made from them will generally be found to give a longer transmission band or pass other colours to a greater or less extent, and the limits of the absorption bands are not often clear-cut. On that account the screens are more or less unsatisfactory, and the only way to determine their suitability is to examine their transmissions with a spectroscope or to make trial exposures. Their transmissions are in many cases very different from what their colour suggests. Screens with sharply defined transmission bands extending over a small part of the spectrum can be obtained by careful combination of screens, or by incorporating two or more dyes in one screen, but as such excellent sets are obtainable at small cost it is seldom worth the trouble of making them.

It is also impossible by inspection to judge of the transmissions of ordinary coloured glasses, but, if suitable pieces of orange, green, blue, or red glass can be obtained, there is no more convenient colour screen to be had than a circle which will fit the ring provided for stops beneath the substage condenser. If used in that position it is much more necessary that the glass be optically flat than if placed at a distance from the condenser.

Liquid Filters.-Solutions are in certain respects better even than glass. They pass more light, a larger range of colours may be made, and they can be more easily adapted to the subject in hand by addition of the colouring matter or by dilution as required, and several very useful solutions are available which cannot be used to dye gelatine. In use they are unfortunately not quite so easy to handle, and many workers on that account 
dispense with them altogether in favour of gelatine and glass. The strength of solution required naturally varies very much with the thickness of the containing vessel and the intensity of the illuminant. Metallic salts can be dissolved in water until a saturated solution is obtained; the amount of solid that a certain quantity of water will dissolve differs for each, but if a few crystals remain at the bottom of the bottle, after due time has been given for solution with frequent shaking, the solution is of maximum strength. Dyes may generally be used in the proportion of 1 part of the dye to 1000 parts of water. These strong solutions are required in narrow cells up to about a centimetre in width, they should be diluted for use in larger vessels.

Filter Diagram.-A diagram of the absorptions and transmissions of a few of the more important solutions is given opposite. The unit of wave length used on the scale is the ten-millionth of a millimetre, the Angström unit, often called the "tenth metre." Wave lengths are also expressed in micra (thousandths of a millimetre), $\mu$, and in millionths of a millimetre, $\mu \mu$. The wave length of the yellow D line of sodium may, for instance, be expressed in any of the following ways, of which the first is here adopted :-

$$
\begin{array}{cc}
\lambda=5896 \cdot 16 \quad \text { A.U. or t.m. } \\
\text { or } & 589 \cdot 616 \mu \mu \\
\text { or } & .589616 \mu \\
\text { or } & 5 \cdot 89616 \times 10^{-5} \mathrm{~cm} .
\end{array}
$$

The absorptions given are those obtained when a Nernst lamp is used with solutions of the strengths mentioned in cells of $\frac{1}{4}^{\prime \prime}$ width. The only solution whose preparation is not clear from the description given in the table is the ammoniacal copper sulphate, and this is made by adding sufficient strong ammonia to the saturated copper sulphate solution to dissolve the precipitate at 


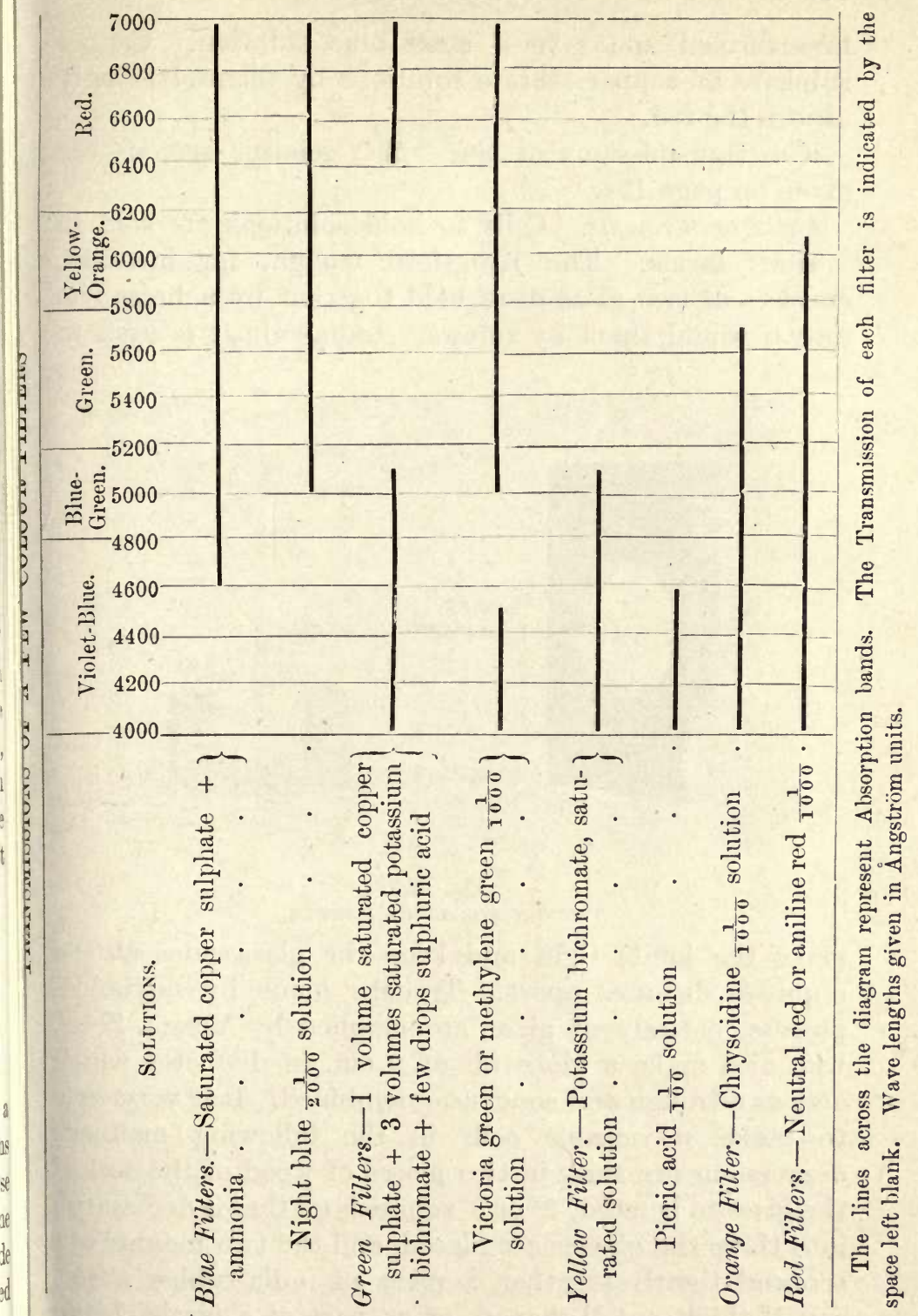


first formed and give a deep blue solution. Copper sulphate or copper acetate solutions by themselves only absorb the red.

The transmissions of the "M" gelatine screens are given on page 174 .

Cells or Troughs. - Cells to hold solutions are sold in various forms. The Kingsford trough, for instance, consists of two glass dises held together by a brass rim drawn round them by screws. India-rubber is used to
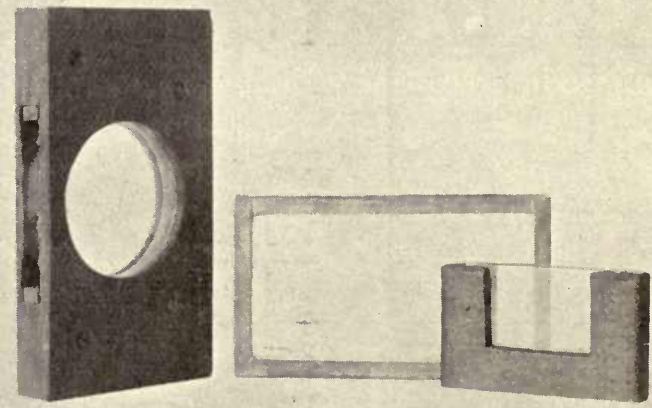

Fig. 49

TROUGHS FOR LIGHT FILTERS

make the joints tight and keep the glass sides at the requisite distance apart. Troughs made by Leybold's process, entirely of glass, are supplied by Messrs. Zeiss, who also make a globe about $9 \mathrm{~cm}$. in diameter which acts as a trough and condenser combined. It is very easy to make serviceable cells in the following manner: depressions are sunk in two pieces of wood of the size of the glass to be used, $2^{\prime \prime}$ or $3^{\prime \prime}$ square is all that is necessary ; into these the glasses are placed, and the two mounts are screwed tightly together, a piece of india-rubber sheet, say $\frac{1}{4}$ "thick, cut V-shaped, or as part of a circle, being 



\section{Direct Colour Photomicrographs.}

Screen plate processes. Autochrome and Paget's duplicating.

A Hoof of Horse $\times 6$

Objective ... ... Seibert's No. 0 Achromat. $36 \mathrm{~mm}$. focus.

Eye-piece ... ... None.

Condenser ... Abbe, front lens removed.

Illuminant ... Limelight.

Aux. Condenser Bull's-eye.

Screen $\quad \ldots \quad \ldots$ Lumière's special screen for limelight.

Plate $\quad \ldots \quad$... Lumière's Autochrome.

Exposure $\ldots \quad \ldots 5 \mathrm{sec}$.

Positive $\quad \ldots \quad \ldots$ The original plate reversed.

B Horizontal Section of Human Scalp $\times 45$, showing the hair follicles.

Objective $\ldots$... Zeiss $16 \mathrm{~mm}$. Apo.

Eye-piece $\ldots \ldots$ Zeiss projection $\times 4$.

Condenser .. Watson's Universal, front lens removed.

Illuminant ... 4 amp. arc.

Aux. Condenser Biconvex lens, parallel light.

Screen $\quad \ldots \quad \ldots$ Wratten's $\mathrm{K}_{1}$.

Plate $\quad \ldots \quad \ldots$ Paget, duplicating process.

Exposure ... ... $1 \mathrm{sec}$.

The positive from which this reproduction was made, is on a Paget special transparency plate, developed with Hydroquinone, and bound up in register with a viewing screen. 


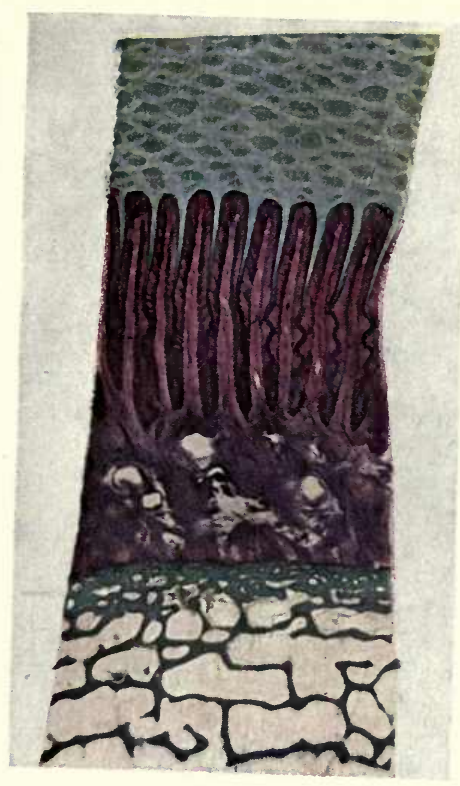

A

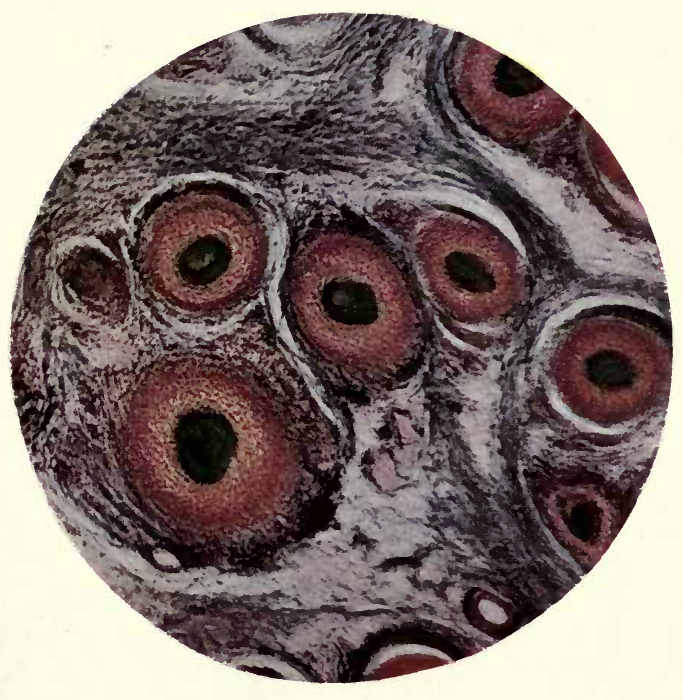






\section{Illustrating the use of Colour Screens in the Photo- micrography of Coloured Sections.}

'The sections were the same as those shown on Plate 18 in their actual colours.

Hoof of Horse (longitudinal section) $\times 7$

Seibert's No. 0 objective, $36 \mathrm{~mm}$. No eye-piece. Watson's Achromatic condenser, back combination. Incandescent gas. No auxiliary condenser. Barnet ortho. plate.

A Red screen, Wratten's A. Exposure, $10 \mathrm{~min}$.

B No screen. Exposure, $\frac{1}{2} \mathrm{~min}$.

Section of Human Scalp (horizontal section) $\times 45$

Seibert's No. 0 objective, $36 \mathrm{~mm}$. Watson's holoscopic eye-piece $\times$ 10. Watson's Universal Achromatic condenser. Liliput arc lamp. Bull's-eye condenser, parallel light. Wratten's Panchromatic " $M$ " plate.

C Wratten's B +E screens, giving yellow-green monochromatic light of wave length 5600-6000 A.U. Exposure, 15 secs.

D Wratten's A+D screens, giving red monochromatic light extending from 6400 to red end of spectrum. Exposure, 15 secs.

$\mathrm{A}$ and $\mathrm{B}$ were taken on orthochromatic plates.

$\mathrm{C}$ and $\mathrm{D}$ on panchromatic plates.

A The effect of the red screen is noticeable, making the green and blue-purple parts of the section, to which it is complementary, dark, while the red parts become light, although the plate is not red sensitive.

B The red parts of the section are darkest on account of the sensitiveness of the plate being least to the light they transmit. The green parts are over-exposed.

C The green screen emphasizes the difference in colour between the orange and red parts of the hair follicles, the contrast being greatly increased on account of the more complete absorption of the light transmitted by the red parts.

D The light given by the red screen more nearly approaches the transmission of the follicles, and the contrast between red and orange is reduced. The structure of the connective tissue is better shown by the red light. 


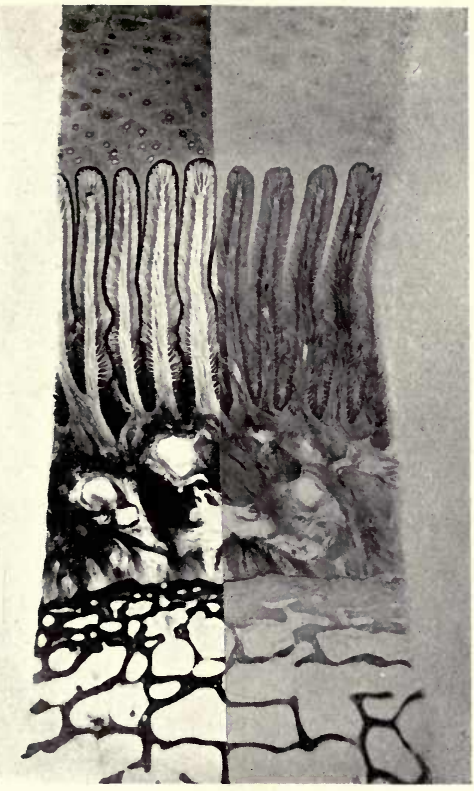

A

B

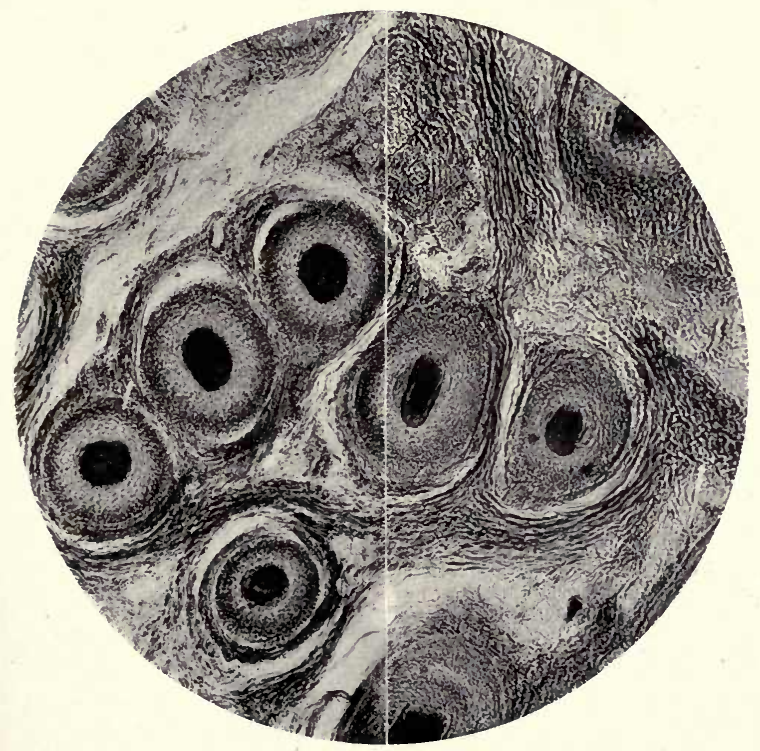

c

D 

placed between the two glasses. Fig. 49 illustrates two of these, and another in which the glasses are bound together in a zinc frame with aquarium cement.

The Use of Colour Screens.-The main objects for which it is necessary to modify the colour of the light by light filters are to ensure :-

1. Good definition and resolution.

2. Adjustment of contrast in the photomicrograph.

3 . Correct colour rendering.

Good definition and Resolution.-The connection between resolution and wave length was mentioned on page 61 , and the improvement produced in a photomicrograph by using blue or green light was noted. Green screens are usually the best for this purpose as the spherical corrections of the objective are most accurate for green light, and also because the light they transmit is more luminous than that passed through a blue screen, and enables an accurate focus to be obtained more readily.

Adjustment of Contrast.-This is often the most important use to which colour screens are put. However accurately an exposure may have been gauged, and in spite of the most careful use of the best optical equipment, there is often a lack of contrast in the negative between the object and background unless a suitable colour screen is used with a suitable plate. This is generally the case if the object possesses only a faint colour, or is faintly stained, particularly if the stain is blue.

On the other hand, the contrast between the background and the object may be excessive, but detail in the object lacking. This is most marked in deep yellow preparations or in those objects which have been stained a deep red; and occasionally the contrast between different parts of an object requires reduction.

It is frequently desirable to emphasise certain parts 
of a preparation, or one colour over the other in doublestained sections. Colour screens are useful for all these purposes.

The degree of contrast in the photomicrograph depends on the colour of the object itself, and on the sensitiveness of the plate to the various colours of the spectrum, and has little or nothing to do with the performance of the lens. Fortunately the means adopted to overcome any lack of contrast in many cases obviate the results of aberrations, and improve definition and resolution at the same time.

Increase of Contrast.-The greatest contrast in a photograph is obtained when part of the subject is black and part white, so that any details or parts of a microscopic object illuminated by transmitted light will stand out in the greatest possible contrast to the surrounding parts if they can be made to transmit no light and appear black. How such a state of things may be brought about will perhaps be best understood if the object is considered for the moment as a light filter passing a definite band of colour depending on the stain used in its preparation. For instance, suppose a section is stained with picric acid and has an absorption corresponding to that of the dye which is indicated on page 159 as extending from the blue end to 4600 . Then suppose an ammoniacal copper sulphate filter is placed in the course of the beam of light before it reaches the preparation. This solution has an absorption which exactly covers the transmission of the picric acid, and the effect will be that the section and screen together will transmit no light and appear black, and the condition for the greatest contrast between object and background in the photomicrograph is thus attained.

Two screens, such as ammoniacal copper sulphate and picric acid solutions, whose transmissions together make up a complete spectrum band, are known as "comple- 



\section{The use of Colour Screens for Contrast and Detail.}

Fairy Fly, Gonotocerus $\delta \times 25$.

Objective ... ... Zeiss Planar. $25 \mathrm{~mm}$.

Condenser ... Back combination of Watson's Universal.

Illuminant $\quad$... Liliput 4 amp. arc, alternating current.

Aux. Condenser Bull's-eye, for parallel beam.

Plate $\quad . . . \quad$... Wratten's "M" Panchromatic.

Screen A Wratten's A+D deep red light 6400 to red end.

B Wratten's D+H violet light 4200-4600.

Exposure A $40 \mathrm{sec}$.

B $10 \mathrm{sec}$.

The contrast in transparency between the delicate wings and the yellowish-brown body, antennæ, and legs of the Fly is considerable, although the preparation is a very fine one. If no screen is used it is difficult to get detail in the body without almost obliterating the wings through over-exposure.

A To reproduce the detail in the coloured portions as well as possible without over-exposing the wings, the photomicrograph is taken with monochromatic red light.

B To get the maximum contrast between the body and wings and background, monochromatic violet light approximately complementary to the yellow-brown of the body is used. 


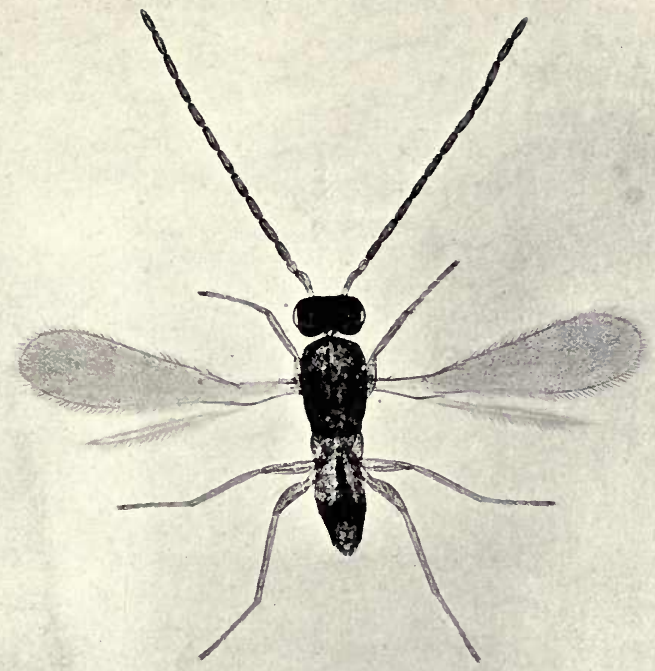

A

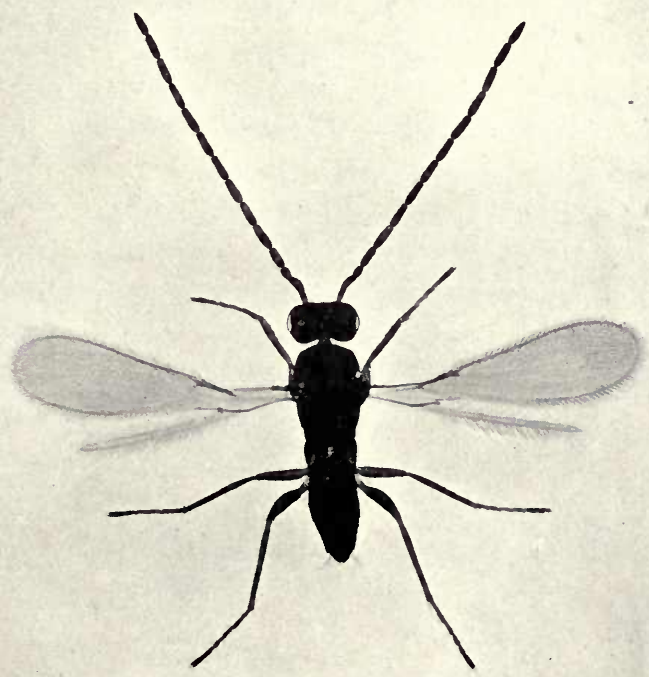



mentary," and one will absorb all light transmitted by the other.

Such accuracy in the adjustment of object and filter can hardly ever be attained, and the opacity of the object is often of much more importance than its colour in determining the absorption of light.

Decrease of Contrast and Increase of Detail.-Increase of detail is particularly required in preparations transmitting yellow or red light, which, being non-actinic, may in extreme cases appear uniformly black in the photograph. Small differences in these colours give details which are easily discerned by the eye, but they may be entirely missed by a plate which reacts mainly to the blue rays, as these are absorbed practically to the same extent by all parts of the object. In order to obtain detail the photograph must be taken by light approximating in colour to the transmission of the preparation, and this involves the use of an orange or red screen and a plate sensitive to orange or red. If there are any colourless or faintly coloured parts. in the preparation they may be so over-exposed as to be entirely lost. before the detail in the yellow part is registered. By using a dark orange or red screen the difference in actinic property of the light passing the yellow and colourless parts of the preparation is so reduced that it is possible to get detail in the one before the other is hopelessly over-exposed. In any case the excessive contrast is much reduced, and whether the two parts will print sufficiently well when a mean exposure is made, depends upon the latitude of the plate, and the amount of over-and under-exposure given to the two parts. If a filter can be found that reduces the errors of exposure in proportion to that actually given, sufficiently to bring them within the latitude of the plate, a print giving detail in all parts of the specimen can be obtained.

Correct Colour-rendering is not often of great import- 
ance in photomicrography as it does not necessarily tend to give the contrast that is so essential. In colour photomicrography, on the other hand, it is of the first moment, and whenever it is required a screen, usually yellow, must be very carefully selected to counteract the greater actinic effect of the blue ; and in this connection the proportion of blue to other colours in the light, and relative sensitiveness of the plate used to the different colours, must be carefully considered. These factors are discussed on page 165 .

The following rules governing the use of filters can be stated; their application will be made clearer by a study of the examples given on Plates 17, 19, and 20.

\section{Rules for the Use of Colour Screens}

1. To Improve Resolution and Definition.-(a) Use a monochromatic screen with a transmission in the yellowgreen corresponding to best corrections of the objective.

(b) In certain cases, when light of very short wave length is desired, use a monochromatic blue screen; this is generally not so satisfactory as $(a)$ with achromatic objectives.

2. To Increase Contrast.-Use a screen complementary in colour to the object, or to the part of the object that it is desired to make darkest in the photomicrograph.

3. To Decrease Contrast or Increase Detail.-Photograph by light corresponding in colour to the transmission of the object, or the part in which detail is required. This is most useful for yellow or red preparations.

4. To get Correct Colour-rendering.-Use a screen properly adjusted to the light and plate, to absorb just the correct amount of the more actinic rays, in order that all the colours of the spectrum may act on the plate in the proportion of their visual luminosities.

Colour of the Illuminant.-In adjusting the colour of 
the light used to the object under examination, the colour screen is not the only factor: the nature of the illuminant must also be taken into consideration, as different radiants have different spectra, or emit the various coloured rays in different proportions. The nearest approach to sunlight that can conveniently be used is the electric arc; the majority of artificial illuminants differ from it in the greater proportion of green, yellow, and red rays compared to the blue which they emit. That this is so can very easily be seen by examining a colourless object under the microscope, using a light blue screen, first with daylight, and then with oil. With daylight it appears blue, with oil-light green, owing to the excess of yellow over blue rays in the radiant. Similarly a double- or triple-stained specimen has an entirely different appearance when examined with artificial light and daylight. Consequently it is essential to treat the illuminant and screen together as a complete light source : any change in the illuminant necessitates a complementary change in the screen if the same result is to be obtained.

Colour Sensitive Plates.-The examples given of the use of colour screens introduce the colour sensitiveness of the plate as a factor in the case, as it is obviously useless to attempt to photograph with red light on a plate that is only sensitive to blue. If a red screen is required to get the best results from a certain preparation, it may be possible to get a better photograph on a yellow sensitive plate with the screen in position than can be obtained without the screen, but the full advantage offered by the red screen cannot be made use of unless a red sensitive plate is employed. If taken on an orthochromatic plate the exposure will be much lengthened, and is really taken by the blue and green light that passes the screen, and not by the red at all.

Plates are broadly divided into four classes according to their colour sensitiveness. 
1. Non-colour Sensitive or Ordinary Plates.-Sensitive to violet and blue only.

2. Orthochromatic Plates.-More or less sensitive to green and yellow as well, and made by incorporating certain red dyes, such as erythrosin, in the emulsion used for ordinary plates.

3. Non-screen, Non-filter, Auto-screen, etc-Orthochromatic plates which have been further dyed in such a way that their blue sensitiveness is reduced. They give the same effect as an orthochromatic plate used with a pale yellow screen to cut off some of the blue light.

4. Panchromatic Plates.-As nearly as possible sensitive to the whole spectrum, and made by bathing the finished non-colour sensitive plates in certain dyes, for example, the isocyanines.

In many cases the enhanced sensitiveness of the plate to colours other than blue will suffice in itself to adjust the contrasts in the negative. And generally, when there is very little colour difference or contrast between parts of the object or between the object and background, a panchromatic plate is very much superior to any other kind for recording these differences, and giving a negative with detail and contrast. Even an orthochromatic plate shows to great advantage over ordinary plates in these and other cases, but to a less extent than a panchromatic.

The four photographs on Plate 21 will serve to show how very dependent the proper realisation of contrast between differently coloured parts of an object is on the character of the plate used. They were taken in the way described on page 128 , with a $7^{\prime \prime}$ anastigmat lens.

There are, therefore, three factors to be considered simultaneously :-

The constitution of the light emitted from the radiant. The transmission of the screen.

The colour sensitiveness of the plate.

So that to eliminate the blue rays, for instance, in the 
most effective way, a radiant deficient in blue rays should be used with a screen transmitting light at the red end of the spectrum, and a plate sensitive to red. And to a certain extent control over contrast may be had by a suitable selection of the illuminant. In practice this is not usually nearly so convenient as control by colour screens, but the effect of a change from a white to yellow light should not be overlooked, and in this connection the following considerations should also be kept in mina, although fairly obvious.

A screen capable, for instance, of cutting off both yellow and green rays, would have no advantage over one cutting off the yellow only, if the radiant did not emit green rays, or if the plate were insensitive to green; and a screen that is able to cut off all the yellow from a light of low intensity might be quite inadequate to do so with a brighter light.

Choice of Screen.-The readiest way to decide what screen to use is to examine the preparation under the microscope with various colours, and select the one which gives the best result, the greatest contrast, the greatest amount of detail, or the best resolution, as may be desired. Inspection in this way is, however, not always a satisfactory guide, as the transmissions of screens are not always such as their colour would suggest. Many yellows and greens, for instance, have incomplete absorptions in the blue and violet, and blue dyes often give a red transmission band. Methylene blue, for example, shows two such bands, one from the red end to 6800 , and another between 6200 and 6500 . The photographic result obtained when such screens as these are used would not be quite what was desired, and in order to determine their transmissions with certainty a spectroscope must be employed.

Focussing when Screens are Used.-It is essential to focus the object with the screen in position when achro- 
matic objectives are used, but an apochromat should give a sharp photomicrograph if the focus is found in white light and the screen then inserted before exposure. A colour screen that does not pass sufficient light to focus by must be rejected; this difficulty arises more especially when using blue filters.

Exposure with Screens.-The increase of exposure necessary when using colour screens is rather difficult to decide, except by experiment. It depends both on the colour and amount of light transmitted, and on the plate used. The matter is discussed in the next chapter. 



\section{Comparison of Ordinary and Colour Sensitive Plates.}

Burnet Мoth : Upper-wings, bronze-green with crimson spots; underwings, crimson with black border.

Common Blue Butterfly.

Swallow-Tall: Yellow and black, with blue markings and red "eye" on under-wings.

Photographed as described on page 128 with a Goerz 7 " anastigmat. The reproductions are reduced from originals of natural size.

A Ordinary plate. Wratten's "Instantaneous."

The crimson-black and bronze-green are not differentiated. Blue is given as white. Yellow is too dark.

B Orlhochromatic plale. Wratten's "Allochrone."

The plate is just sufficiently sensitive to bronze-green to show the black spots on the wings in contrast to it.

Red still appears black, but blue is slightly lower in tone than with an ordinary plate and yellow is lighter.

C Panchromatic plale. Wratten's "Panchromatic."

All detail is shown; in particular crimson is rendered light in accordance with its visual luminosity.

The yellow is the high light. Blue is still too light, but rather darker than in B.

D Orlhochromatic plate and yellow sereen. Allochrome plate and " $\mathrm{K}_{: 3}$ " screen.

Both Panchromatic and Ortho. plates are proportionately much more sensitive to blue and violet than to other colours, and the best possible colour rendering is only obtained by absorbing all the ultra violet and a suitable proportion of the more actinic rays from the blue end of the spectrum by a yellow screen. Under these conditions a correct rendering of the whole range of colours is obtainable on a Panchromatic plate.

This figure shows the effect of a $\mathrm{K}_{3}$ screen on an Ortho. plate. Blue takes its correct grey in the monochrome scale. The green on the upper wings of the Burnet and the yellow are made lighter, but red is not affected. 

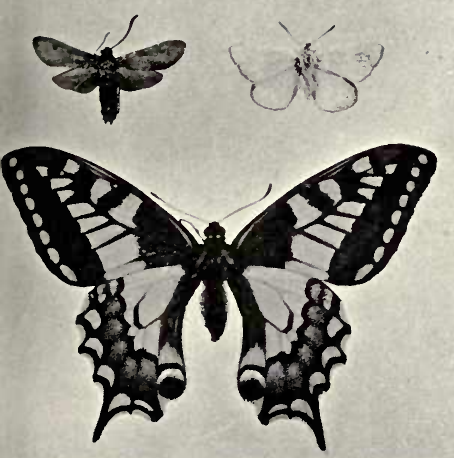

A
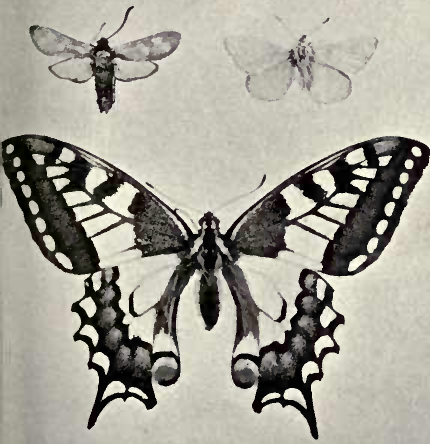

C
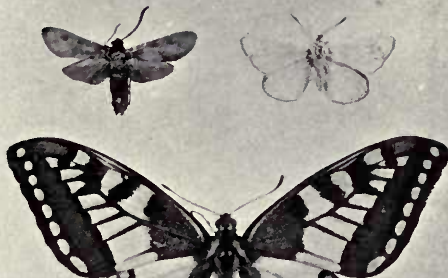
Shan

B
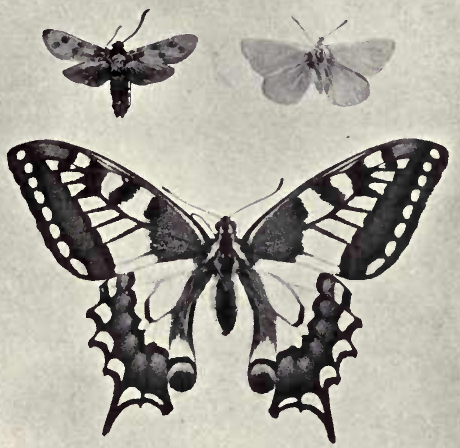

D 



\section{CHAPTER $\mathrm{X}$}

\section{EXPOSURE}

THE quality of a negative depends very largely on the accuracy of the exposure given, though errors discovered before development can be counteracted to a large extent by suitable modification of that process. It is, however, impossible to give any very definite instructions to cover all cases, the times required are modified by so many different factors. Actinometers and sensitometers are made to measure the actinic value of the light and the rapidity of plates, but they are not satisfactory when using colour sensitive plates and colour screens.

Trial Exposures.-When there is doubt as to the exposure required the best way to decide on the time is to make trial exposures in the following way. Arrange the object and illuminant, and focus on the ground glass. Make as near an approximation to the required exposure as experience or appearance on the ground glass permits, say 10 seconds. Then insert the plate, draw the slide, and give an exposure of half the time thought to be correct, that is 5 seconds. Push the slide in one quarter of its distance and expose again for 5 seconds more. Then push it in another quarter and expose a further 10 seconds. Finally, push it in another quarter and expose 20 seconds longer. On development for the correct time demanded by the developer used, four strips of different densities appear on the negative, corresponding to the different exposures given to the four quarters of the plate, 5,10 , 20 , and 40 seconds respectively. The time corresponding 


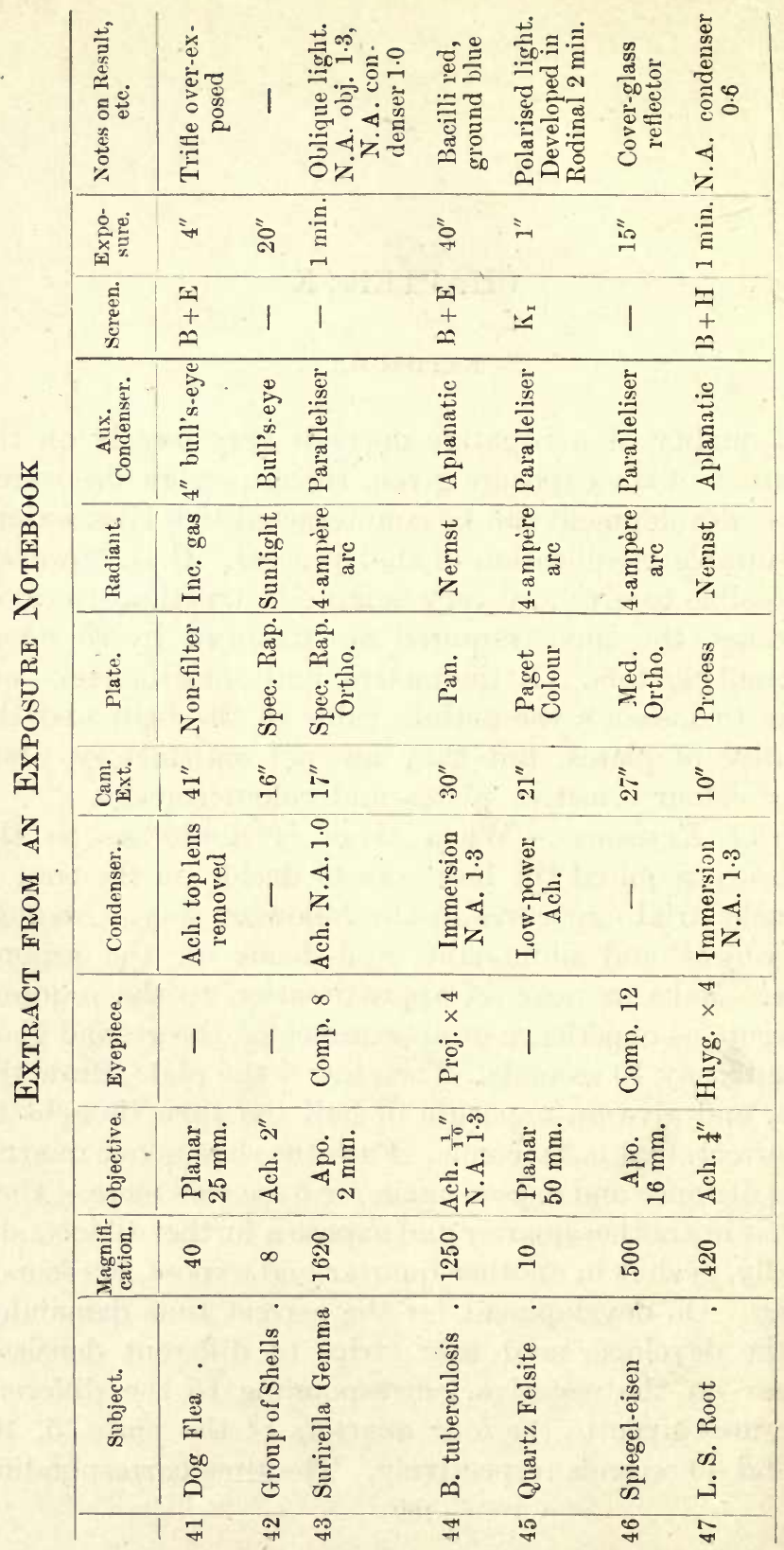


to the strip of best printing density is the correct exposure for the subject, plate, and light used. Trial exposures should always be in geometrical progression, and not with equal intervals between them.

Record of Exposures.-Experience enables one to judge with very fair accuracy the exposure required under any given conditions, but particulars of all photomicrographs taken should be entered in a notebook at the time they are made, with details of subject, light, lenses, screens, magnification, and exposure. In particular, making a note of any peculiarities in the subject or errors made. A systematic entry of such details as those indicated in the accompanying excerpt from an exposure notebook is of the greatest assistance even to an experienced worker, who can turn back at any time, when in doubt, for the particulars of work previously done of a similar nature to that for which he requires guidance. The best magnification to give, the best combination of lenses or aperture of condenser to use, the most suitable screen to employ, can be more easily decided upon when the results obtained in previous experiments are recalled.

Every negative is numbered when made either by writing on the glass side with a diamond, or on a corner of the film side in pencil or ink. If pencil is used the figure should be written on the plate before development, as it takes the writing more easily then.

Factors Influencing Exposure.-The most important factors which govern exposure are the following :-

1. Light source ...... Intensity and colour.

2. Colour screen ...... $\left\{\begin{array}{c}\text { Spectral transmission and } \\ \text { transparency. }\end{array}\right.$

3. N.A. of objective.... Exposure varies as $\frac{1}{(\text { N.A. })^{2}}$. 
4. Magnification...... $\left\{\begin{array}{l}\text { Exposure varies as (magnifi- } \\ \text { eation) })^{2}\end{array}\right.$

5. Condensing system... Amount of light utilised.

6. Immersion medium.. $\left\{\begin{array}{l}\text { Air or oil between condenser, } \\ \text { objective, and slide. }\end{array}\right.$

7. Eyepiece ........ \{ High eyepieces require a largely increased exposure. 8. Object......... $\left\{\begin{array}{c}\text { Its density, contrasts, colour, } \\ \text { and mounting medium. }\end{array}\right.$

9. Plate........... Speed and colour sensitiveness.

Systematised Exposure.-A worker who does not greatly vary the conditions under which he makes his exposures can give definite values to some of these factors, and arrive at a fairly accurate exposure time by multiplying them together, and then multiplying his unit of exposure by the product; the unit of exposure being the time required under certain definite conditions.

As an example some tables are appended, taken by permission of Messrs. Wratten and Wainwright from their booklet Photomicrography, referring to their "M" panchromatic plates and " $M$ " screens. Other plates with different colour sensitiveness, and screens with greater or less absorptions, will require different factors, which can be determined by experiment.

Effect of N.A.-Exposure varies as $\frac{1}{(\text { N.A. })^{2}}$ :-

\begin{tabular}{r|c|c}
\hline Oljective. & Average N.A. & Exposure Factor. \\
\hline $2^{\prime \prime}$ or $50 \mathrm{~mm}$. & $\cdot 15$ & 10 \\
$1^{\prime \prime}$ or $24 \mathrm{~mm}$. & $\cdot 25$ & 4 \\
$2^{\prime \prime}$ or $16 \mathrm{~mm}$. & $\cdot 35$ & 2 \\
$\frac{1}{2}^{\prime \prime}$ or $12 \mathrm{~mm}$. & $\cdot 45$ & $1 \frac{1}{4}$ \\
$1^{\prime \prime}$ or $8 \mathrm{~mm}$. & $\cdot 50$ & 1 \\
$\frac{1}{4}^{\prime \prime}$ or $6 \mathrm{~mm}$. & $\cdot 8$ & $\frac{2}{5}$
\end{tabular}




\begin{tabular}{l|c|c}
\hline \multicolumn{1}{c|}{ Objective. } & Average N.A. & Exposure Factor. \\
\hline$\frac{1}{6}^{\prime \prime}$ or $4 \mathrm{~mm}$. & .85 & $\frac{1}{3}$ \\
$\frac{1}{8}$ or $3 \mathrm{~mm}$. & .9 & $\frac{1}{3}$ \\
$\frac{1}{12}$ or $2 \mathrm{~mm}$. & $1 \cdot 0$ & $\frac{1}{4}$ \\
do. with objec- & & \\
tive and con- & & \\
denser oiled to & $1 \cdot 3$ & $\frac{1}{8}$ \\
slide. & & \\
\hline
\end{tabular}

Effect of Magnification.-Exposure varies as (M)²:-

\begin{tabular}{c|c} 
Magnification. & Exposure Factor. \\
10 & $\frac{1}{100}$ \\
25 & $\frac{1}{16}$ \\
50 & $\frac{1}{4}$ \\
100 & 1 \\
250 & 6 \\
500 & 25 \\
1000 & 100
\end{tabular}

Effect of Light Source.-This factor varies very greatly with the colour sensitiveness of the plate used. The figures given are based on those required by Wratten's special photomicrographic panchromatic plate "M." Those in the columns headed "Orthochromatic" and "Ordinary" refer to plates of the same speed in daylight, namely, 100 Watkins or $68 \mathrm{H}$ and D. The "Allochrome" plate would require the orthochromatic factors to be multiplied by $\frac{2}{5}$, as its speed in daylight is $2 \frac{1}{2}$ times as great as that of the " $M$ " plate, but if orthochromatic plates of other make are used, the simple ratio of their speed numbers in daylight will not necessarily be correct, as their greater or less sensitiveness to yellow, etc., is of much greater importance, so that the factor for each must be determined by experiment. The column headed 
"Ordinary" gives approximately the factor for any non-colour sensitive plate of medium speed.

\begin{tabular}{c|c|c|c}
\hline Light Source. & $\begin{array}{c}\text { Factor for } \\
\text { “M” Plate. }\end{array}$ & $\begin{array}{c}\text { Orthochro- } \\
\text { matic. }\end{array}$ & Ordinary. \\
\cline { 1 - 2 } Oil . . . . . . . . . . & 1 & 4 & 8 \\
Incandescent gas . & $\frac{1}{3}$ & $\frac{3}{4}$ & $\frac{4}{3}$ \\
Nernst (1 amp.) .. & $\frac{1}{12}$ & $\frac{1}{5}$ & $\frac{1}{3}$ \\
Acetylene . . . . . . & $\frac{1}{12}$ & $\frac{1}{6}$ & $\frac{1}{4}$ \\
Direct arc (4 amp.). & $\frac{1}{50}$ & $\frac{1}{33}$ & $\frac{1}{33}$ \\
do. (30 amp.) & $\frac{1}{1000}$ & $\frac{1}{700}$ & $\frac{1}{700}$ \\
\hline
\end{tabular}

The most noticeable feature is the very great advantage of panchromatic plates so far as speed is concerned with the yellower illuminants. The figures are only approximate, and vary with the particular lamp employed.

Exposure Factor of the " $M$ " Screens with Various Illuminants.-These factors refer to the panchromatic " M" plates, and will, of course, not hold for orthochromatic plates or any others of different colour sensitiveness. They are given for the single screens, and for pairs used together to give monochromatic bands. Their spectral transmissions are given in Angström units.

\begin{tabular}{|c|c|c|c|c|c|c|}
\hline Screen. & Transmission. & $\begin{array}{l}\text { Factor } \\
\text { for Oil }\end{array}$ & Nernst. & Arc. & $\begin{array}{l}\text { Incand. } \\
\text { Gas. }\end{array}$ & $\begin{array}{l}\text { Acety- } \\
\text { lene. }\end{array}$ \\
\hline A scarlet. & red end to 5900 & 3 & 6 & 6 & 6 & 5 \\
\hline $\mathrm{B}$ green & $6000-4600$ & 12 & 12 & 12 & 12 & 12 \\
\hline $\mathrm{C}$ blue-violet & $5100-4000$ & 25 & 16 & 12 & 12 & 12 \\
\hline E orange. & red end to 5600 & 2 & 3 & 6 & 6 & 4 \\
\hline F pure red & red end to 6100 & 6 & 6 & 8 & 12 & 8 \\
\hline G strong yellow & red end to 5100 & $1 \frac{1}{2}$ & 2 & 4 & 4 & 3 \\
\hline $\mathrm{H}$ blue . & $5400-4200$ & $24^{\circ}$ & 16 & 12 & 16 & 16 \\
\hline $\begin{array}{l}\mathrm{K}_{3} \text { (for orthochro- } \\
\text { matic reproduction) }\end{array}$ & 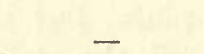 & $1 \frac{1}{2}$ & $1 \frac{1}{2}$ & $1 \frac{1}{2}$ & 3 & 2 \\
\hline$A$ and $\mathrm{D}$ deep red. & red end to 6400 & $60^{2}$ & $90^{2}$ & $240^{2}$ & 240 & 120 \\
\hline $\mathrm{B}$ and $\mathrm{E}$ yellow-green & $5600-6000$ & 120 & 60 & 250 & 120 & 90 \\
\hline $\mathrm{G}$ and $\mathrm{H}$ pure green. & $5100-5400$ & 1000 & 1600 & 1600 & 1600 & 1600 \\
\hline $\mathrm{B}$ and $\mathrm{C}$ blue-green & $4600-5100$ & 1000 & 600 & 600 & 1000 & 800 \\
\hline $\mathrm{I}$ and $\mathrm{H}$ violet. & $4200-4600$ & 200 & 150 & 64 & 160 & 90 \\
\hline$B$ and $G$ green . & $5100-6000$ & 25 & 25 & 64 & 20 & 30 \\
\hline
\end{tabular}


The variation of these factors with the nature of the illuminant is very noticeable. Liquid screens of the same spectral transmission have, as a rule, lower factors.

Unit of Exposure for the Above Tables.-To determine the exposure for any object under definite conditions, make an experiment with a good clear specimen, using an oil lamp and no screen at 100 magnifications, with an objective of .5 N.A. on an "M" plate. Suppose that 10 seconds proves to be the correct exposure.

Then for any other conditions, so far as they are covered by the tables, the exposure required is

10 seconds $\times$ factor for N.A. $\times$ factor for light

$\times$ factor for magnification $\times$ screen factor.

To take an example. An object is to be photographed

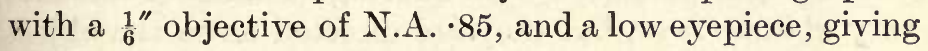
a magnification together of 250 times. Green light by screen B, 1-ampère Nernst lamp, and " $M$ " plate used. The exposure will be

\section{0 seconds $\times \frac{1}{3} \times \frac{1}{12} \times 6 \times 12$}

\section{$=20$ seconds.}

This method of working out exposures from a consideration of the individual factors governing it is rather complicated, but it is the most satisfactory method when making any change in arrangement. The basis is a definite exposure determined under the conditions usually employed. As a rule a worker does not employ a wide range of different conditions, and it is fairly easy to arrive at a unit of exposure for the plate commonly used with each of the screens employed; the other factors for magnification and N.A. are then very rapidly made use of. More frequently still, previous experience has decided a correct exposure under certain conditions, and the alteration made necessary by a change in one of them is all that is required. 


\section{CHAPTER XI}

\section{OBLIQUE AND DARK GROUND ILLUMINATION,}

CINEMATOMICROGRAPHY-POLARISED LIGHT

Oblique Illumination.-The methods of illumination hitherto described all depend on the employment of an axial cone of light, and great stress has been laid on the correct "centration of the condenser. The wide-angle cone of light projected by a substage condenser includes very oblique rays, but they are directed on to the object from all sides equally. What is usually understood as "oblique illumination" is obtained by stopping out the greater part of the cone of light, and allowing a pencil from a portion only of the peripheral zones of the condenser to impinge on the object. The perfection of the image given by a microscope depends on the numerical aperture of both the objective and condenser, and consequently it can hardly be expected that by obscuring a great part of the aperture of the condenser an accurate representation of minute structure is obtained, and, in fact, the results must always be regarded with great caution, as false images, which may be easily taken for a true representation of the structure under examination, are very liable to be produced. Oblique illumination is, however, sometimes employed in the resolution and study of very minute periodic structures such as occur in certain diatoms, though whether the appearance shown represents the true structure of the diatom or is only a diffraction effect must be decided by other methods of research, one of which is afforded by ultra-violet light, 



\section{Direct Colour Photomicrograph.}

Screen-plate process (Dufay).

Group of Diatoms $\times 56$

Dark ground illumination.

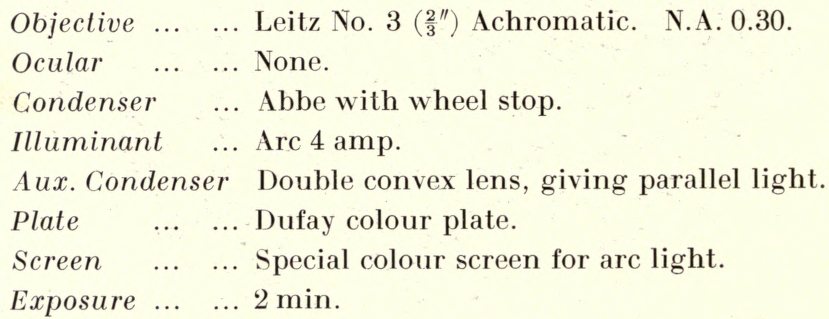

The diatoms are colourless with transmitted light, but show varie and delicate tints, due to interference phenomena, when examine under dark ground illumination. 


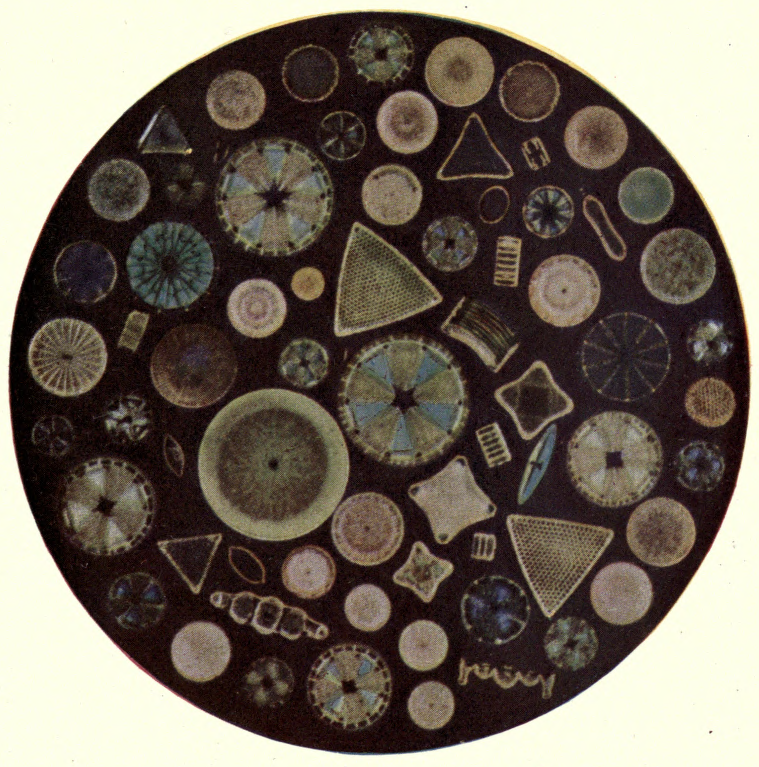






\section{Dark Ground Illumination of Transparent Objects.}

A wheel stop placed beneath sub-stage condenser.

A FaIRY FLY $\times 50$

B Group of Sponge Spicules $\times 50$

Objective ... ... Leitz No. 3. $16 \mathrm{~mm}$. Ichromat.

Ocular ... ... Leitz No. 3.

Condenser $\quad$... Abbe, with wheel stop.

Illuminant ... Limelight.

Aux. Condenser Zeiss Köhler condenser, parallel light.

Plate $\quad \ldots \quad \ldots$ Warwick.

Exposure ... ... 5 sec.

Developer ... Pyro-soda.

Davis nose-piece diaphragm used. 


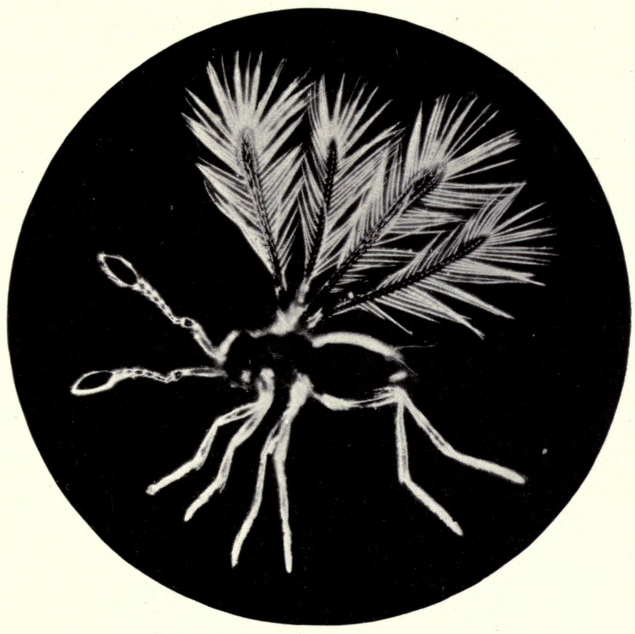

A

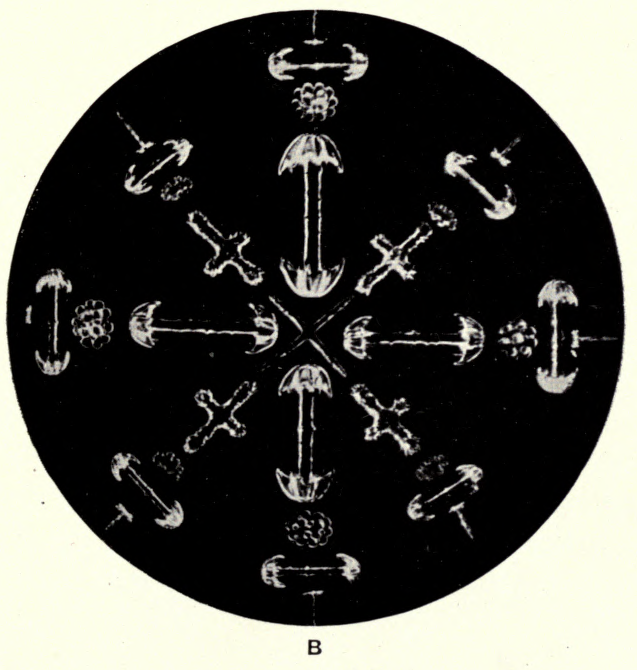



which gives much greater resolution than can be obtained by ordinary light.

The Continental substage is particularly well-adapted to give oblique light in any direction that may be desired, by racking the iris diaphragm to the edge of the ccndenser and rotating it until illumination is in the desired azimuth. The control over the aperture of the diaphragm and over its position is so complete that the best adjustment can be obtained while the object is actually under examination. Patch stops of various shapes, two of the most useful of which are shown at Fig. 50, are required to give oblique illumination with the English substage. They are dropped into the ring and rotated until the aperture is in the desired position; or the direction of the
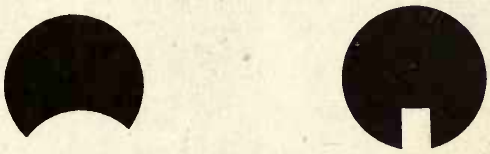

Fig. 50

STOPS FOR OBLIQUE ILIUMINATION

light in reference to the object is adjusted by rotating the stage instead.

The photomicrograph of Amphipleura pellucida (Plate 43) may be cited as an example of the use of oblique light. When examined with a wide-angle cone of direct light, and an oil-immersion objective, this diatom is resolved into parallel lines at right angles to its length. It is difficult, however, to obtain a photograph in this way, but if one of the stops, preferably the second, shown in Fig. 50, is placed in the substage ring with its aperture in what may be conveniently described as the " north " or "south," while the diatom lies "north and south," these transverse lines are made more distinct, and the photomicrograph can then be taken with comparative facility, although even then a considerable amount of skill is 
required to get a good result. If the stop is then rotated until its aperture is in the "east" or "west," longitudinal lines appear, while if it is in the "north-west," "south-east," etc., resolution into dots instead of lines is sometimes obtained. The procedure just described is that always employed with oblique illumination, the beam of light is caused to fall perpendicularly on to the direction of the lines to be resolved or emphasised.

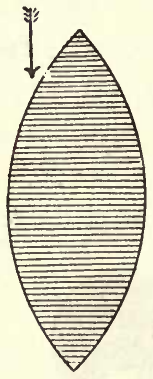

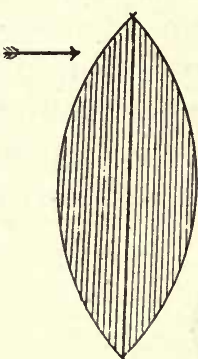

Fig. 51

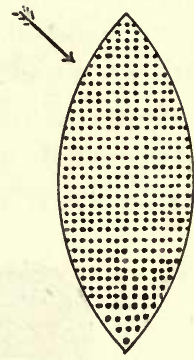

RESOLUTION BY OBLIQUE LIGHT

Fig. 51 illustrates diagrammatically the procedure adopted to resolve the details of such diatoms as $\mathrm{Am}$ phipleura pellucida or Navicula rhomboides. Navicula is the simpler, and the resolution can usually be effected

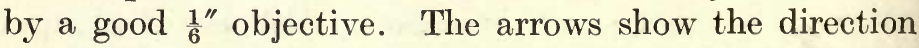
of the oblique light necessary to resolve the diatom as indicated.

Amphipleura pellucida is often considered as a good test object for oil-immersion objectives, but the resolution into dots with oblique light depends more on the specimen than on the objective, and, if a suitable specimen can be obtained, an objective that cannot claim to be first-class will resolve it, but such specimens are rare. Navicula rhomboides and Surirella gemma can be resolved into dots in axial light, but the dots appear with greater contrast when oblique is employed. 
An oil-immersion condenser or an Abbe oiled to the slip should always be used when experiments are to be made with oblique light, in order that rays of sufficient obliquity may reach the objective.

Dark Ground Illumination.-Opaque objects are easily mounted and illuminated in such a way that they are exhibited on a dark ground, but the method described in this chapter is that employed to display transparent preparations in a similar manner. By using a hollow cone of light, whose apex is in the plane of the object, the particles of the preparation are made to appear as if selfluminous in a black field. To ensure a completely black field, and sufficient contrast between it and the object, no light must be allowed to pass directly from the condenser to the objective, the light forming the image being diffused from the object itself. The effects produced are in many cases very beautiful, but. until recently the scientific interest attached to this method of illumination was small. Since the introduction of new dark ground illuminators, suitable for use with high-power objectives, its usefulness has greatly increased, particularly in the domain of bacteriology. Organisms and flagella, ordinarily almost invisible, are rendered distinctly on a black field, and can be detected and photographed in a living state, whereas, heretofore, difficult methods of preparation and staining were essential.

Dark Ground Illumination by Central Stops.-The spot lens and Wenham's paraboloid were formerly employed to produce dark ground illumination, and from them the new illuminators have been evolved. The earlier forms did not prove satisfactory on account of the chromatic aberrations they introduced, and because the use of any one was limited to low-power objectives of a N.A. to which the amount of central light it cut off was suited. However, good pictures can be obtained when they are employed, though a much better method, for use with 
an ordinary substage condenser, is to place a metal or cardboard stop under the condenser to block out the central rays of light and to permit the passage of the peripheral rays only. The size of this stop must be adjusted both to the focal length of the condenser and the N.A. of the objective, in order to cut out entirely the dioptric beam and yet leave a sufficiently large hollow cone to illuminate the object brilliantly. The stop must be large enough to occlude an aperture slightly larger, say $10 \%$, than the N.A. of the objective, and its diameter is determined, according to a rule given by Dr. Spitta in Microscopy, by multiplying the equivalent focus of the condenser by twice the N.A. to be stopped out. They are

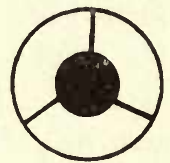

Fig. 52

WHEEL STOP

made in the shape shown in Fig. 52, and placed in the ring beneath the condenser, or they can be made with a central pin-point, over which dises of correct size for the objective in use are placed. The Traviss expand-

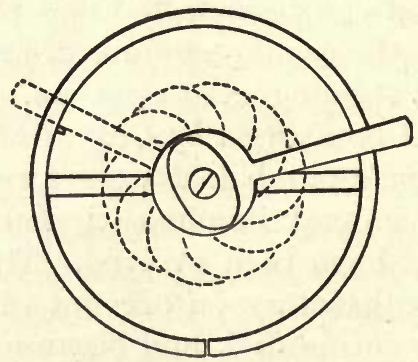

Fig. 53

TRAVISS' EXPANDING STOP

ing stop, Fig. 53, is very useful for the purpose, as the size of the stop can be adjusted to a nicety by moving 
the lever while the object is actually under examination.

The diagram Fig. 54 shows the course of the rays

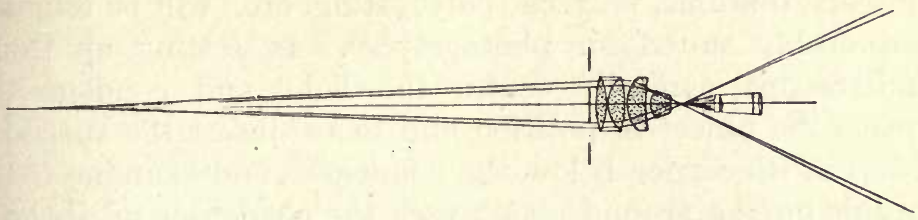

Fig. 54

DIAGRAM OF DARK GROUND ILLUMINATION BY CENTRAL STOP

through Watson's universal condenser when a central stop is placed beneath it. As only the marginal rays are used to illuminate the object, and they do not enter the objective, the image is formed by rays diffused from the object only, and exposure is rather long unless a powerful illuminant is used. The condenser must also be of considerably greater aperture than the objective; for a $\frac{1}{6}$, for instance, it is almost essential to use an oil-immersion condenser, and its entire aperture must be illuminated by means of an auxiliary condenser. It is advantageous to have at hand some means of decreasing the N.A. of the objective; an internal conical stop is now supplied for use with $\frac{1}{12}$ " oil-immersion objectives, but it is usual with lower powers to employ a Davis iris diaphragm.

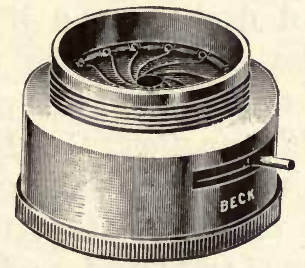

Fig. 55

DAVIS' DIAPHRAGM

(Fig. 55) between the objective and tube of the microscope. 
Dark Ground Illumination for Low Powers.-There are a great number of objects that may be most beautifully displayed by this method with a central stop; many insects, diatoms, polyzoa, polycystina, etc., will be found admirably suited for photography. In setting up the microscope, carefully centre the light and condenser, place the object in position and focus, insert the special stop in its carrier below the condenser, and examine the result on the ground glass : rack the condenser up if the best result is not obtained. If the background is not sufficiently dark try a larger stop or shut down the iris of the Davis diaphragm until the best result is produced. Colour fringes are frequently produced around the object, as imperfections in the optical system are made most apparent. If they occur a sharp negative is sometimes impossible unless a monochromatic screen is used. In other cases the production of interference colours gives a most beautiful effect without any lack of sharpness in the negative. Plate 22 shows the appearance often presented by diatoms under these conditions of illumination. It is essential that the slide be scrupulously clean, as any particles of dust, scratches, etc., will show up as brilliant points and lines of light, or as diffraction effects, and ruin the negative.

The photomicrographs on Plates 23 and 24 are given to illustrate the use of dark ground illumination for displaying some of the smaller forms of natural objects which come within the scope of low-power work.

Dark Ground Illumination for High Powers.-The method just described is unsatisfactory for high powers unless a condenser of the most perfect chromatic and spherical corrections is employed, and Leitz has recently introduced a more highly corrected modification of his oil-immersion condenser with the special object in view of providing an illuminator suitable for all purposes.

Greater brilliance and more complete freedom from 



\section{Dark Ground Illumination.}

A

Lophopus crystallinus

$\times 20$

Objective ... ... Crouch $2^{\prime \prime}$ Achromat.

Ocular

Condenser ...

Illuminant ...

Aux. Condenser

Plate ... ...

Exposure ... ...

\section{Leitz 0.}

Spot lens.

4 amp. arc.
$6 \mathrm{sec}$.
B

Group of Polycistina

$\times 45$

Leitz $3,16 \mathrm{~mm}$.

None.

Abbe with wheel stop. Limelight.

Double convex lens, giving parallel light.

Imperial Special Rapid.

$2 \mathrm{sec}$.

The spot lens referred to is a plano-convex lens of about $1 \frac{1}{2}{ }^{\prime \prime}$ to $2^{\prime \prime}$ focal length, with the top surface partially obliterated by a black patch.

A Davis nose-piece diaphragm was used for both photomicrographs and closed down until the best result was obtained.

Lophopus, one of the fresh-water Polyzoa, is an almost clear gelatinous organism forming a very beautiful object for dark ground illumination. The slide was mounted by Clarke \& Page in a special fluid which, to a great extent, preserves the natural appearance of the Polyps. In the photomicrograph several individuals are seen extending from the common body.

Polycistina are fossil siliceous skeletons of minute marine unicellular organisms (Protozoa); by deposition as Radiolarian oozes and ultimate consolidation they have built up rock masses in Barbadoes and other parts of the world. 


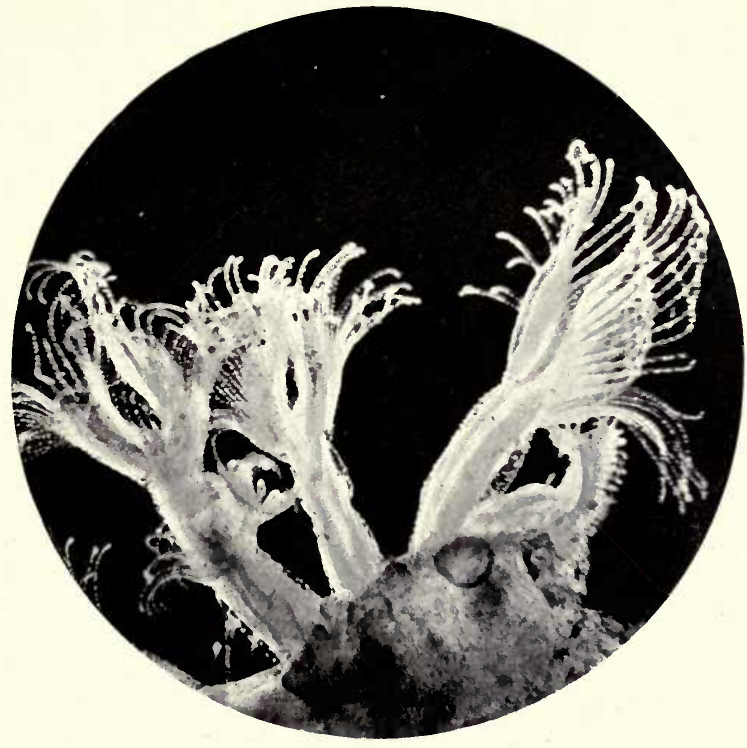

A

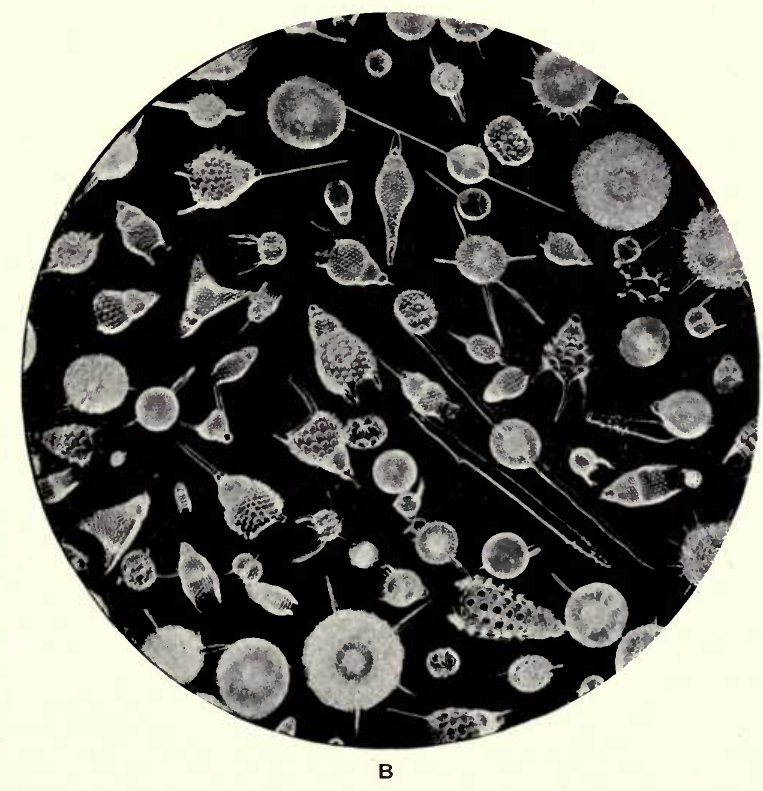



chromatic effects are even then obtained by the use of the new dark ground illuminators, in most of which light is passed to the objective by reflection only. Fig. 56 shows

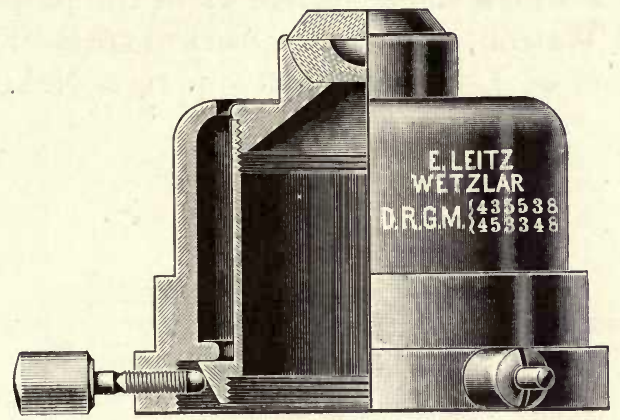

Fig. 56

LEITZ' DARK GROUND ILLUMINATOR

Leitz' dark ground illuminator. The Nelson dark ground condenser, Fig. 57, made by C. Baker, involves refrac-

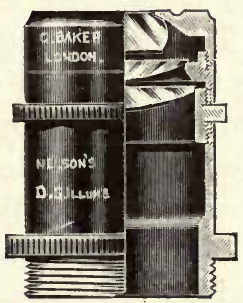

Fig. 57

NELSON'S DARK GROUND CONDENSER

tion, but the results given by it compare very favourably with those given by the reflecting illuminators. Its corrections may not be so accurate as those of some of the reflectors, but it is easier to work with, as it is not quite so sensitive to errors in centration, and does not require so intense an illuminant for photographic purposes.

The reflecting condensers of different makers vary in construotion, though the principles involved are the same. 
A central stop prevents the passage of light direct to the objective at any angle less than that corresponding to a N.A, of 1 ; beyond this, light is admitted to the illuminator, and reflected at a single surface as in the paraboloids of Zeiss and Watson, or at two spherical surfaces as in the illuminators of Leitz and Swift, up to a N.A. of about

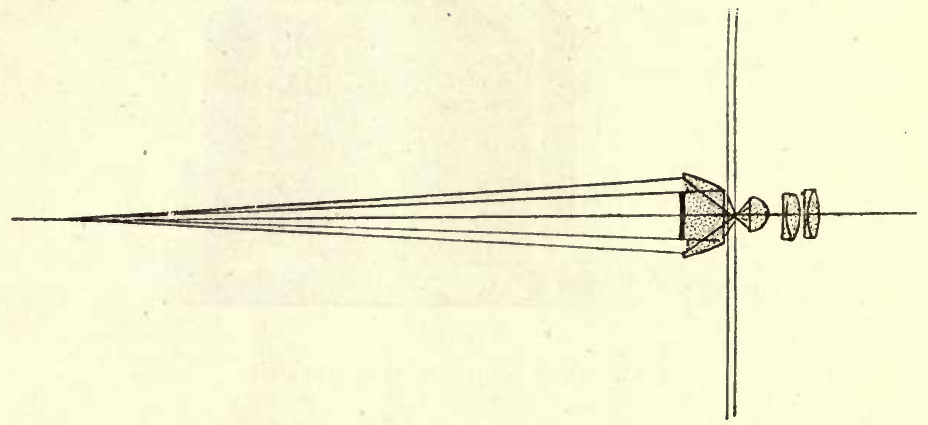

Fig. 58

DIAGRAM OF ILLUMINATION BY WATSON'S PARABOLOID

1.35 or 1.4 . Fig. 58 shows the course of rays in the Watson paraboloid. The light diffused from the object is represented as filling the aperture of the objective, but it is not possible to show what becomes of the direct light as each ray is reflected back at the under surface of the cover-glass in exact coincidence with a corresponding ray from the opposite side of the paraboloid, the light, as it were, returning upon itself.

The reflecting surfaces are so accurately shaped in several of these illuminators that spherical aberrations are practically eliminated, and the rays are brought to a point with great nicety, as indicated in the diagram of the passage of light through the Leitz dark ground illuminator (Fig. 59). As a consequence it is necessary that the microscope-slides used with these condensers be of such thickness that the focus of the rays is in the plane of the object: the required thickness is given with each make of condenser, and is generally about $1 \mathrm{~mm}$. 


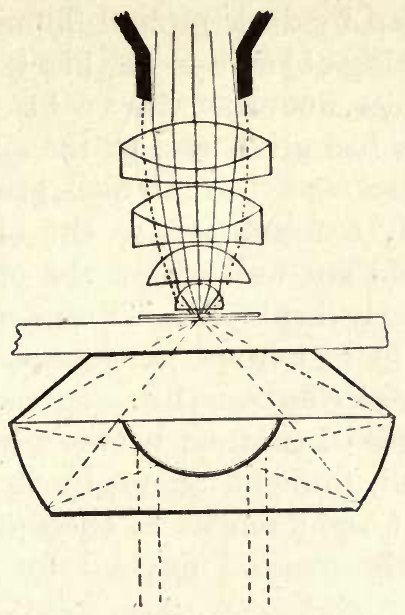

Fig. 59

COURSF OF RAYS IN LEITZ' DARK GROUND ILLUMINATOR

In order that the rays, which, as indicated in the figure, are very oblique, shall not suffer total reflection at the under surface of the slip, the latter is always oiled to the condenser. The light consequently passes through to the object, and when a dry objective is used, any rays that may reach the cover-glass are totally reflected, and the only rays utilised by the objective are such as are deflected by the particles of the preparation, and emitted at an angle within the aperture of the objective. On the other hand, when the objective is oiled to the cover-glass these oblique rays can pass direct to it, and be more or less utilised according as its aperture is greater than N.A. 1. This would prevent the formation of a dark field, so a conical stop restricting the aperture to 1.0 is screwed into the objective mount with its apex close to the back lens.

The Ultramicroscope.-Small particles in a preparation are made visible in proportion to the contrast with which they are displayed against the background, and the 
contrast produced by dark ground illumination, between the brightly shining object and the black field, is so great, that minute bodies, not otherwise visible, can be seen very distinctly. This has given rise to the application of the term "Ultramicroscope" to the dark ground condensers, but this is really a misnomer, as the objects for which they are intended are well within the ordinary range of visibility if properly displayed. Ultramicroscopy properly refers to much more minute particles, such as are made visible in colloidal solutions by a special application of the same principle of contrast between bright object and dark background, brought about by illumination by a beam of light at right angles to the optical axis of the microscope, in the method devised by Siedentopf and Szigmondy.

Radiants for High-Power Dark Ground Photomicrography.-The contrast necessary to display blood corpuscles, bacteria, and other minute bodies, for which the new condensers are particularly useful, sufficiently brilliantly for photography, is only produced by very intense illuminants, more especially as the particles are generally in fairly rapid movement in a fluid medium. Exposures of about $\frac{1}{25}$ or $\frac{1}{50}$ of a second are then the longest permissible, and are only possible with sunlight, arc-light, or limelight; exposures of 1 to 5 seconds are required by Nernst lamp or incandescent gas combined with a good condensing system. A cooling trough is also frequently essential on account of the deleterious effect of heat on the living organisms.

Adjustments for the Dark Ground Illuminators.-A vertical camera is generally the most convenient on account of the movements produced in a liquid preparation when placed on a microscope in the horizontal position. The mirror is centred with great care, the condenser is oiled, placed in position, and accurately adjusted. This is effected by racking it up until the 
bright spot it produces on the object is as small as possible, this bright spot is then centred with the lowest-power eyepiece in position. The Nelson condenser has a small ring engraved on the centre of its top lens which can be focussed and centred.

The most convenient objectives are $\frac{1}{4}^{\prime \prime}, \frac{1}{6}{ }^{\prime \prime}$, or $\frac{1}{8}$, and apochromats show to great advantage over achromats. Care must be taken to correct for cover-glass thickness, and, as any departure from the best adjustment causes a deterioration in the image, it is well to use the lower-power dry objectives, which are not so sensitive ; magnification can be made up by eyepiecing. The $\frac{1}{12}$ " oil-immersion, with its aperture cut down to 1.0 by means of a conical stop screwed inside the mount, combined with a low eyepiece, is also suitable, and is generally the best achromat to use when apochromats are not available.

As errors of correction in the objective are made rather more apparent with dark ground than with ordinary illumination, it is well to use monochromatic light with achromatic objectives whenever possible. The photography of moving bacteria under these conditions is, however, impossible unless a very powerful illuminant is employed to reduce the exposure.

Air bubbles in the immersion fluid must be avoided, as they effectually cut off the light, and the mount must be as thin as possible, as any particles not quite in focus are very annoying. There must also be no dust or scratches on the cover-glass to show up as bright specks and lines. Care must finally be taken to fill the back of the condenser completely with light, as any restriction of the hollow cone that it gives will very much redice the brightness of the image.

Cinematomicrography.-Dr. Comandon, in conjunction with Messrs. Pathé Frères, has applicd dark ground illumination to the cinematography of living and moving micro-organisms and blood preparations. By the courtesy 
of Messrs. Pathé an example of these films is given on Plate 38 ; by their projection on a screen a most wonderful reproduction of the original object as seen under the microscope is produced. The movements of larger organisms, etc., can be displayed equally well by this or the ordinary methods of illumination, and in many cases the radiant need not be so intense as is necessary for bacteria. For a serial photograph intended for display in a cinematograph at the actual rate of movement of the object, 16 exposures a second should be made, each with a

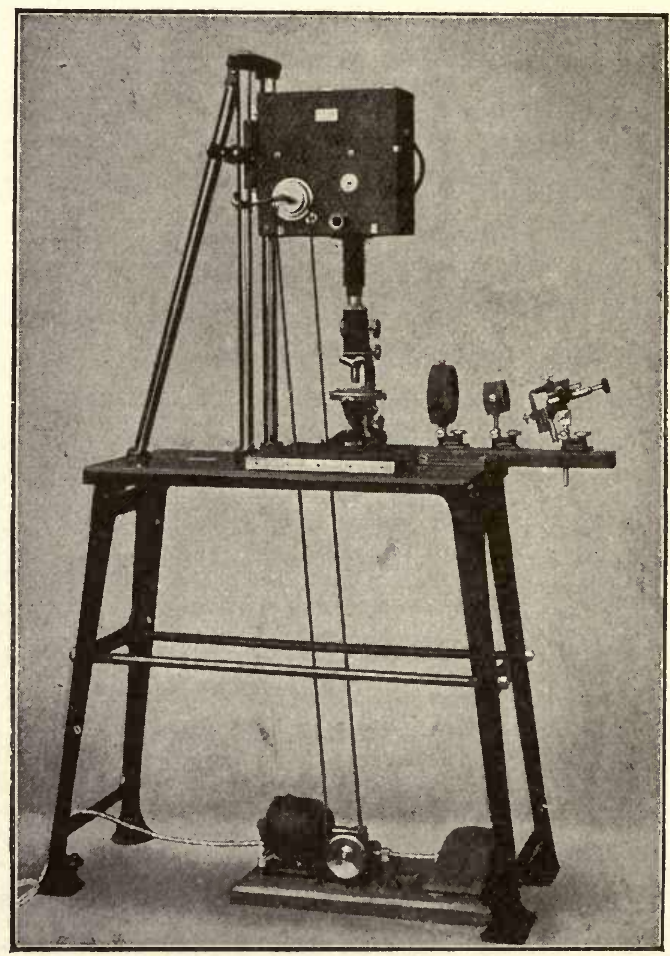

Fig. 60 



\section{Polarised Light.}

A Crystals of Cinchoninine with Crossed Nicols $\times 45$

B Section of Quartz Diorite with Crossed Nicols $\times 20$

A $\quad$ B

Objective ... ... Leitz No. 3 Achromat. Crouch $2^{\prime \prime}$ Achromat. $16 \mathrm{~mm}$. N.A. 0.3 .

$\begin{array}{lllll}\text { Ocular } & \ldots & \ldots & \text { Leitz } 0 . & \text { Leitz } 0 .\end{array}$

Condenser ... Back combination of Watson's Universal Achromatic, placed above the polariser.

Illuminant $\quad . . \quad$ Inc. gas, inverted. Liliput 4 amp. arc.

Aux. Condenser Bull's-eye, covering the lower face of the polariser with a convergent beam.

\begin{tabular}{|c|c|c|c|}
\hline Screen & $\ldots$ & $\cdots$ & Wratten's K 1 \\
\hline Iate & $\ldots$ & $\ldots$ & Wratten's “M”'Pan. \\
\hline
\end{tabular}

$\begin{array}{llll}\text { Exposure ... ... } & 2 \mathrm{~min} & 5 \mathrm{sec} .\end{array}$

Developer $\quad$... Pyro-soda.

An endeavour is generally made to reproduce the appearance of polariscope objects as closely as monochrome tones allow. The yellow screens $K_{1}$ and $K_{3}$ were used with this purpose in view, they were selected in accordance with the conditions of light and plate in conjunction with the colours of the specimen. When reds and yellows largely predominate a Panchromatic plate must be used, but an Ortho. plate is quite satisfactory where blues and greens are the chief colours.

If it is desirable in any case to emphasise one colour in contrast to the others suitable screens can easily be selected. 


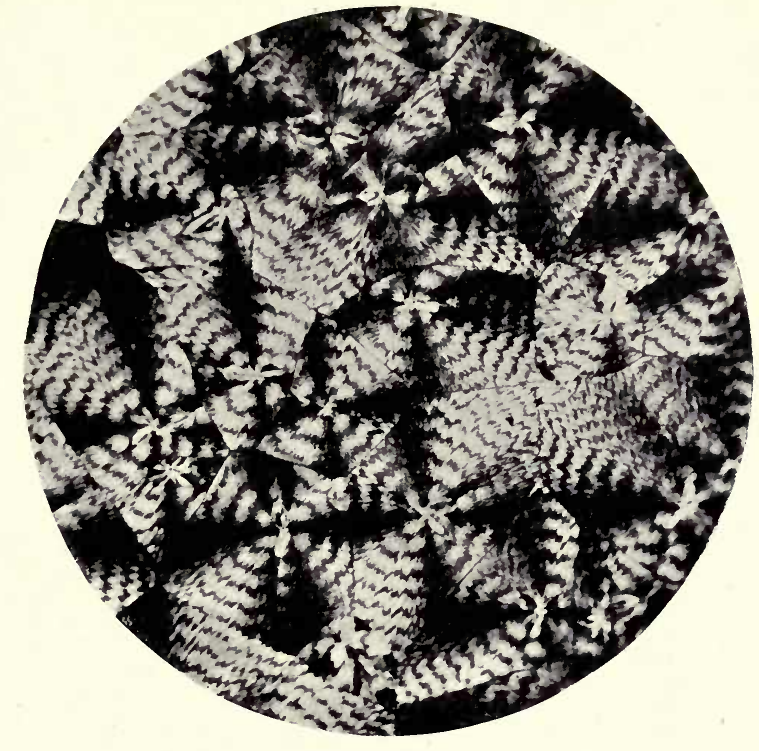

A

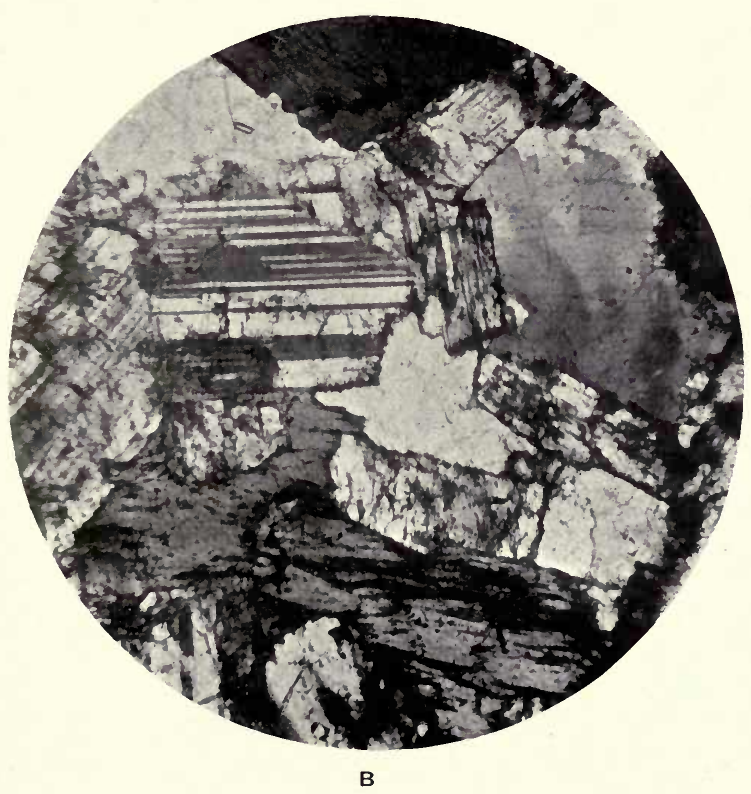



duration of $\frac{1}{3}$ of a second. In the case of bacteria, blood preparations, etc., magnified 400 diameters and illuminated with a dark ground condenser, an arc lamp or limelight and condenser are essential. Fig. 60 shows a cinematomicrographic apparatus by Ernemann. The arc lamp should be automatic, otherwise it is difficult to keep the crater at one point for a sufficient time. A condenser and cooling trough are placed between it and the microscope. The camera is arranged vertically, and fitted with a handle, by means of which it may be swung rapidly into position after adjustment of the microscope and lamp, and when a suitable field has been found. A sidetube is also provided with an arrangement to fit over the eyes so that the image on the film can be watched during exposure. The mechanism for moving the film can be worked by a handle, but a small electric motor geared to its shaft by a belt is preferable.

Photomicrography with Polarised Light.-Polarising apparatus is indispensable on a microscope intended for petrology, and most makers supply special stands fitted with analyser, polariser, selenites, and completely rotating and graduated stage, etc., built on the general lines of their ordinary stands. These are, of course, most desirable when any amount of petrological work is done, but by the addition of a polariser to the substage, and an analyser fitting in a nose-piece or above the ocular, almost any microscope can be used for this purpose. Either the analyser or polariser must rotate, and when the axes of the Nicol's prisms they contain are crossed, no light passes unless certain refractive bodies are in the field of view, in which case the play of colours is frequently not only extremely beautiful, but of use in studying the object. This is one of the cases in which colour photography is particularly applicable, and examples are given on Plates 1,10 , but even prints in monochrome are useful (Plate 25). Obviously the best results cannot be obtained 
unless panchromatic plates are used, without which the differentiation of yellows and reds is impossible. Long exposures are necessary with radiants of low intensity, but a few seconds are adequate with lime- or arc-light. The colour plates, of course, require considerably longer exposure. The particulars accompanying the plates give sufficient indication of the exposures and adjustments required. 


\section{CHAPTER XII}

\section{OPAQUE OBJECTS-METALLOGRAPHY}

The methods of photomicrography as applied to opaque objects may be conveniently considered under two heads, those used when only low magnifications are required, and those employed for high powers. The former find considerable application for natural history objects and the like, and the latter in metallography.

\section{Natural History and other Objects Requiring Low}

Powers.-No microscope is required in many cases, and the methods given in Chapter VII apply; but when somewhat higher magnifications are desirable, and the microscope is employed, more intense illumination is necessary. The method adopted to take the photographs reproduced on Plate 26 was to place the radiant in front of the microscope stage, and to direct a converging beam on to the object by means of a bull's-eye condenser. The beam should impinge on the object as nearly vertically as possible, so as to obtain even illumination, and to obviate as much as possible the production of excessive shadows. But when working with objectives of $\frac{1}{2}$ " or $\frac{1}{3}$ " focal length, the beam of light will of necessity have to be oblique, otherwise a shadow of the objective will be cast on the object itself. This method will be found unsatisfactory for objectives higher than $\frac{1}{3}$.

By placing a light and condenser on each side of the object, more even illumination may be obtained in some cases and shadows may be eliminated to some extent, though the presence of shadows, if not too intense, is 
useful in giving an appearance of relief to the finished photograph. If a powerful illuminant is used, a watertrough should be placed between it and the condenser, or concentration of the heat rays may ruin the slide. When the objects to be photographed are solid and fairly large, it will be found necessary to place a stop in or above the objective to increase the depth of focus. A set of cylindrical metal stops having apertures of $\frac{1}{6}{ }^{\prime \prime}, \frac{1}{12} \mathbf{1}^{\prime \prime}$, and $\frac{1}{2 \bar{z}} "$ in diameter may be made to fit in the objective mount between the component glasses, or the Davis diaphragm can be used for the purpose, and is frequently more convenient, though not quite so satisfactory.

On account of the relatively small amount of light reflected from the object, a very long exposure will be required, particularly if the objective is stopped down to any great extent, and for this reason an intense illumination is necessary for this kind of photomicrographic work. The presence of a cover-glass on the preparation may give rise to reflections, and, whenever possible, should be removed before photographing.

There are other means of illuminating opaque objects, namely, by the use of a parabolic side reflector or a Lieberkuhn; but, as a rule, the results obtained by these are in no way superior to those that can be produced by a careful adjustment of the bull's-eye condenser, and the latter should form part of every microscopist's outfit. One form of the parabolic reflector is shown at Fig. 64 among the accessories supplied with the Rosenhain microscope. It is a little silvered mirror carried in the optic axis of the microscope by a long dovetailed slide which fits into a groove on the body. Light is thrown on to the reflector by means of a condenser, and focussed on to the object by sliding the reflector up or down. The latter nearly touches the object or slide when in position.

Stereoscopic Photomicrography.-The best representation of the forms of small solid objects is obtained by 



\title{
Low-power Photomicrography of Opaque objects displayed on a black background.
}

\author{
A Hind-leg of Honey Bee, inner surface $\times 16$ \\ B Leaping Spider $\times 12$
}
Objective .. ... Crouch $2^{\prime \prime}$ Achromat. Stopped down. Ocular ... ... None.
Condenser ... Bull's-eye projecting convergent beam.
Illuminant ... Incandescent gas.
Plate $\quad \ldots \quad \ldots$ Imperial Special Rapid.
Exposures $\quad \ldots 12$ and 10 minutes.

A small stop placed between the component lenses of the objective to get sufficient depth of focus.

Hind-leg of Bee mounted as a dry preparation on dead black paper in the centre of a glass slip. No cover glass used.

The largest segment of the tarsus of the 3 rd leg is provided on its inner surface with ten rows of stiff hairs, the pollen combs, which are used to comb out the pollen from the hairs on the body and legs. Two pollen grains can be seen adhering to one of the combs.

The spider was completely immersed in methylated spirit in a watch glass lined with black paper. 

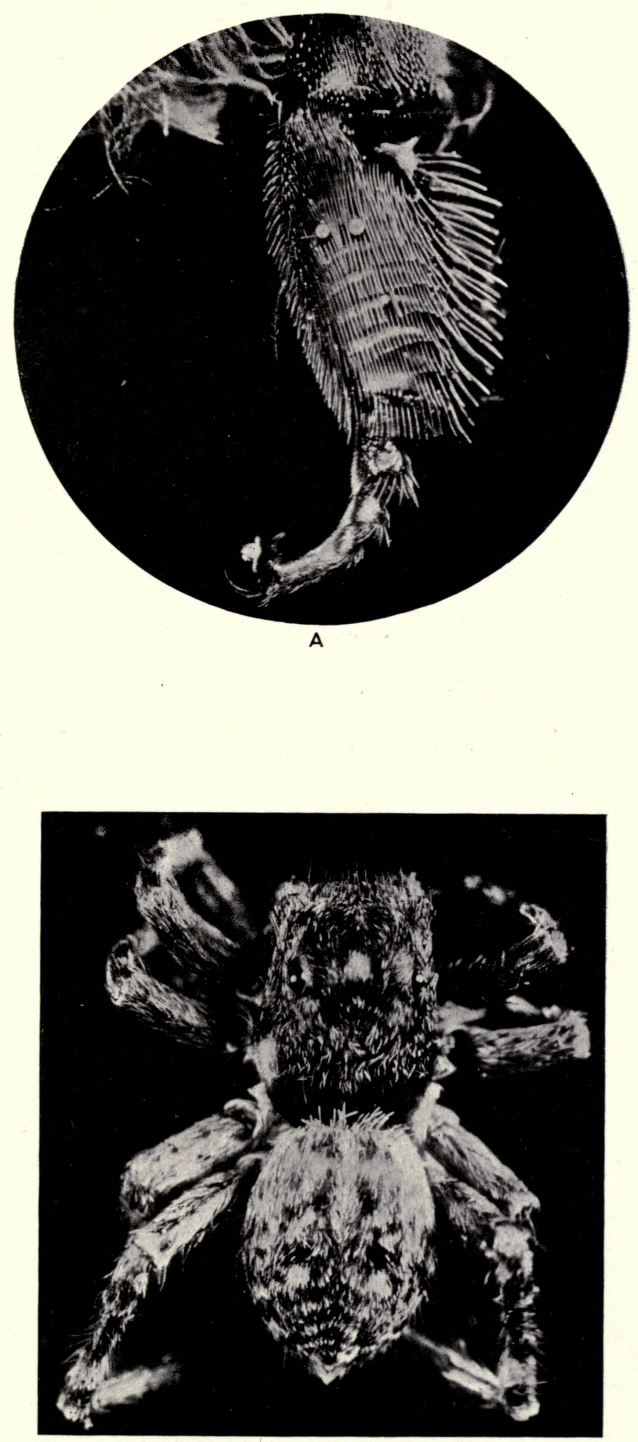




\section{Stereoscopic Photomicrography.}

Grodp of Insect Eggs mounted as Opaque Object $\times 14$

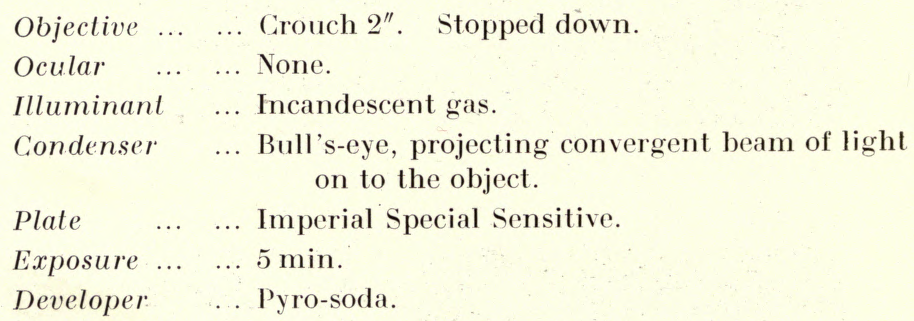

The method employed is as described on page 193, involving a slight movement of the preparation on the stage, and two exposures on a half-plate. 

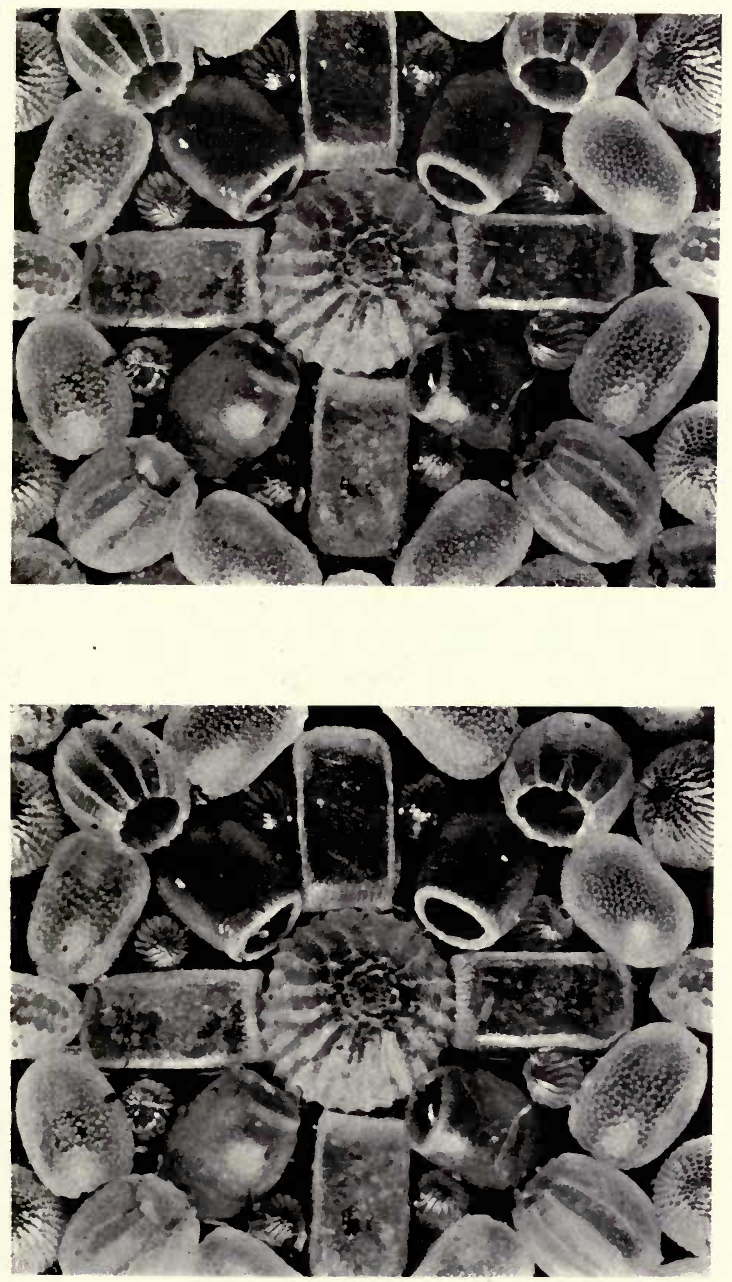

taking two photographs from positions on either side of the perpendicular on the centre of the object, and combining prints or transparencies from the negatives in a stereoscope. If a half-plate camera is used there is no difficulty in getting the two negatives of an object illuminated as above described. Two distinct dots should be made on the ground glass $2 \frac{1}{2}$ " apart, and equidistant from the centre. The object is focussed and so arranged that the centre of the image coincides with one of the dots, and a photograph is taken; the object is then moved until the centre of the image coincides with the second dot and another photograph taken, the exposure given being the same in both cases. Both photographs can be taken on the same half-plate if one half of it be covered by an opaque card previous to the first exposure, after which the slide is taken to the darkroom and the card placed on the exposed part of the plate; the second exposure is then made on the unexposed half. The two images will develop up to the same density if correctly exposed. If it is preferred to use quarter-plates, suitable carriers can be cut out of black cardboard, or dark slides are obtainable constructed to carry two quarter-plates or a special stereoscopic size, and with shutters that permit of two exposures being given. As a rule the condenser and lamp will require readjustment before the second exposure is made, as the movement of the object affects the equality of the illumination. A mechanical stage is of great service for stereoscopic work, as it obviates any difficulty in the movement of the object from one position to the other.

The need for moving the object, with the readjustment of the illumination thereby rendered necessary, can be avoided either by moving the objective on a sliding or centring nose-piece to the extent required to form the two images on the ground glass, or by covering up first one 
half of its back lens and then the other, and making the two exposures under these conditions. The best stop to use for this purpose is one that will just fit over the back lens, and provided with a small circular aperture excentric in position (Fig. 61). After the first exposure this

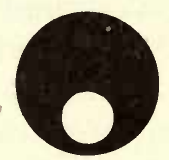

Fig. 61

OBJECTIVE STOP FOR STEREOSCOPIC PHOTOMICROGRAPHY

stop is turned round through $180^{\circ}$ and the second made.

Very beautiful stereoscopic results are obtained with microscopes fitted with two exactly similar objectives, whose axes converge on the object. These microscopes have two tubes to which a camera for two plates is attachable.

Stereoscopic effect necessarily requires a considerable depth of focus, and as generally only low-power objectives are used, this is as a rule easily attained. The circular stops behind the objective help in this way, and a Davis diaphragm is also very useful.

To obtain pleasing results it is well to give fairly full exposures and make moderately soft prints: any very harsh contrasts are unpleasantly obtrusive in the stereoscope, and mar the effect. When the two prints are made on one piece of paper from the two negatives on one plate, it is necessary to cut them and reverse their positions left to right on mounting.

Metallography.-The photography of the minute structure of metals provides one of the most important applications of photomicrography, and most of the refinements devised for the study of opaque objects under medium and high powers have been designed with the 
intention of facilitating "Metallography." Invaluable evidence is thereby obtained of the mechanical or physical treatment to which a metal has been subjected, and as to its state of purity or the nature of its alloyed components, and so forth. The details of the preparation of the surface of the specimen by polishing and etching vary with the nature of the metal. An outline of a useful general process, practically Stead's, is given with Plate 29, others will be found in works on metallography. To get the best results, particularly with medium- and high-power objectives, a beam of light must be projected vertically on to the prepared surface which, to prevent avoidable reflections causing a haze, is not covered with a cover-glass, and consequently dry objectives should be corrected to work under those conditions.

The vertical illuminator in a form that can be adapted to any ordinary microscope consists of a nose-piece which screws into position above the objective, and carries a circle of thin optical-glass or cover-glass adjustable about

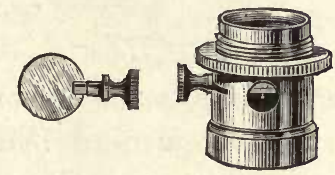

Fig. 62

VERTICAL ILLUMINATOR

a horizontal axis by a small milled head (Fig. 62). A pencil of light is projected by means of a condenser strictly at right angles to the microscope into the vertical illuminator, and reflected thence through the objective, which acts as a condenser and focusses the light on the object. The image-forming rays from the object pass through the reflector without hindrance.

Another form of vertical illuminator contains a reflecting prism in place of a cover-glass circle, and is shown as 
made by Zeiss at Fig. 63. A beam of light is projected through the opening $p$, and reflected by the prism through half of the objective, while the image-forming rays pass up through the other half. It will be noticed that the objective mount in the figure is very short, reflections at the back lens are thereby minimised. The makers also supply stops suited to different powers, which slide into the mount between the prism and objective for the same

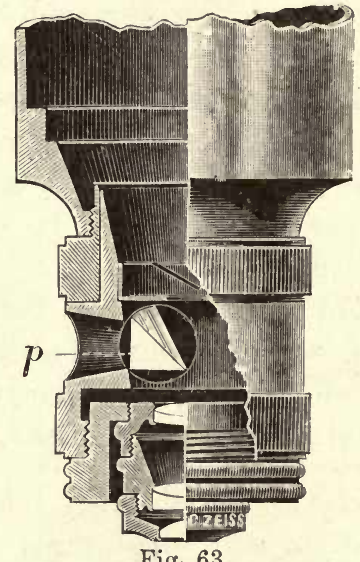

Fig. 63

PRISM VERTICAL ILLUMINATOR

purpose. The prism gives a more brilliant illumination than the cover-glass reflector, and can consequently be used with a less intense illuminant or a higher power objective. There is also less haze over the image of a highly polished metal surface, but the resulting photomicrograph is liable to show an embossed structure in which the crystals stand out as a slightly raised pattern, in contra-distinction to the smooth surface represented when the cover-glass illuminator is used.

Critical illumination is obtained in a manner analogous to that employed for transparent objects. The lamp is placed about two feet from the microscope, and a condenser, preferably with iris, placed in such a position 
as to project an image of the radiant on to the reflector. The objective acting as a condenser forms a minified image of the condenser aperture on the metal surface. Haze-producing reflections at the various surfaces are among the greatest difficulties encountered, and, as a further help to their elimination, the illuminated area on the specimen should be cut down by means of the iris in front of the condenser until it is just that shown in the eyepiece or on the camera ground glass. An iris is also frequently fitted to the nose-piece.

It is obvious that this method of illumination, which depends on the projection of a beam of light into a small aperture in the tube behind the objective, could only be carried out with great difficulty if the objective were focussed on to the object in the usual way. To avoid this difficulty and consequent loss of time, microscopes for metallography are constructed in such a way that the stage can be moved up and down in the optical axis, in place of, or as well as, the tube. On some stands the stage is fitted with a coarse and a fine adjustment, on others the fine adjustment is fitted to the tube, as the very small motions caused by final focussing with it scarcely necessitate any alteration of the position of the beam of light. The stage itself is frequently made in a special manner, it may be a plane stage without any aperture, and so suited for opaque bodies only, or it may be fitted to hold moderately large pieces of metal between suitable jaws which can be turned about to present the desired surface to the objective. And further, certain stands have no stage at all, but are made to clamp on to any large piece of metal that it is desired to examine. Mirrors are frequently mounted above the stage for reflecting light on to the object. Most makers now make a stand embodying these requirements as an elaboration of their ordinary type, and many of them can be used for any class of work, and are not necessarily restricted to metallography. 
Metallurgical Microscopes.-A figure of the Rosenhain microscope, made by Beck, is. given as an example of the

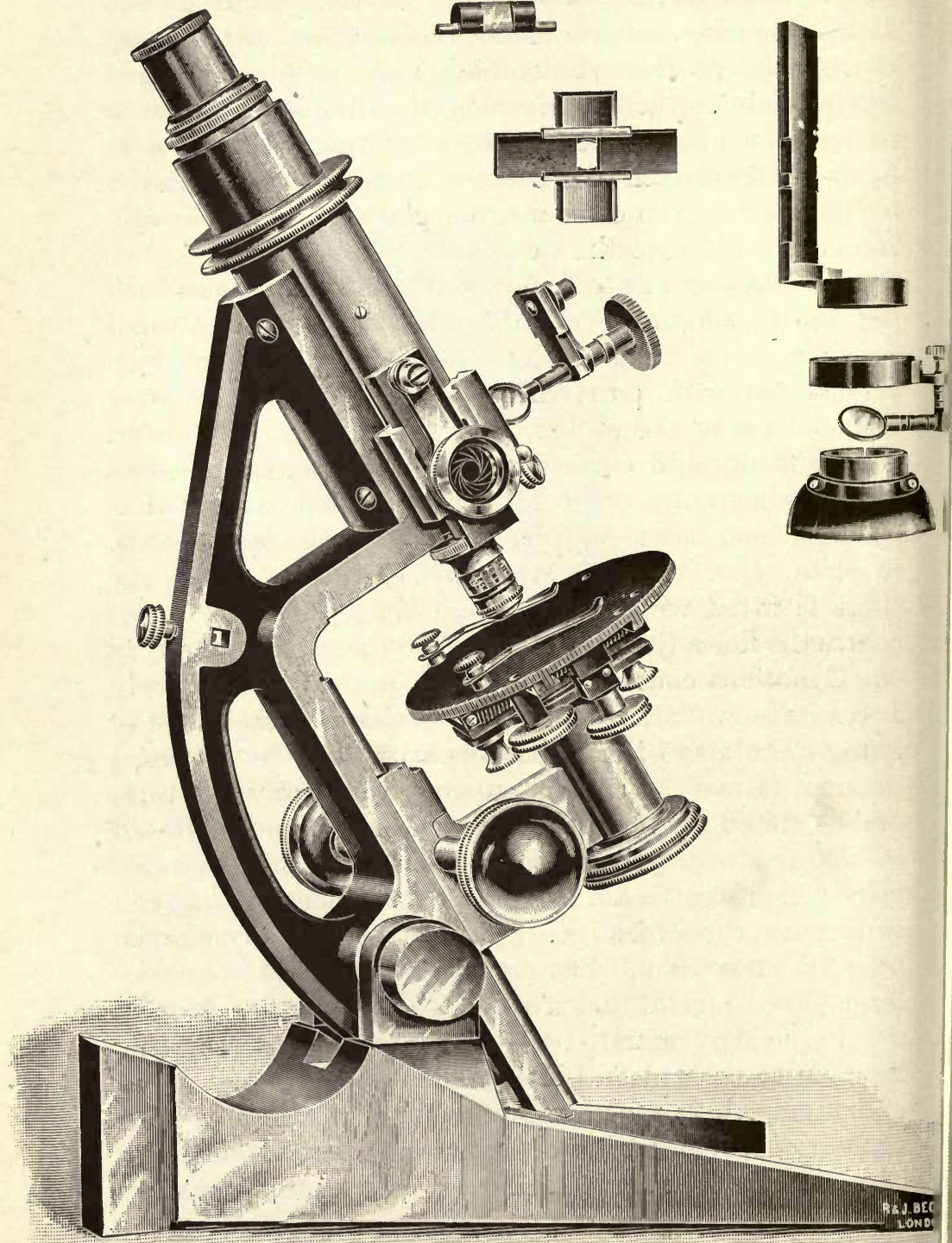

Fig. 64 
specially designed metallurgical stands (Fig. 64). It shows the general principles which guide the construction of these instruments, but has many special features in design, such as the massive foot and limb, which give great stability in any position. The coarse and fine adjustments both actuate the stage. The various illuminating appliances used in metallography are carried in dovetailed slides on the body. Of these, the iris diaphragm is shown in position, the cover-glass and parabolic reflectors at the side. The camera used with metallurgical microscopes may be either vertical or horizontal, but there are microscopes, the Marten's stand made by Zeiss for instance, which work only in a horizontal position.

Adaptation of Ordinary Microscopes to Micro-metallography.-Fig. 65 shows how an ordinary microscope may

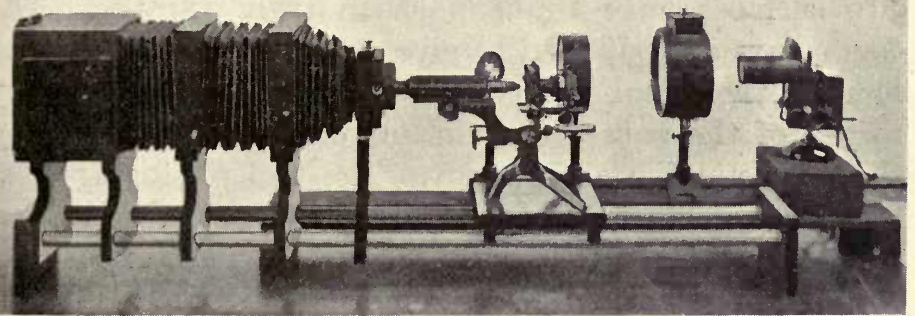

Fig. 65

ARRANGEMENT OF CAMERA FOI MICRO-METALLOGRAPHY

be adapted for photomicrography of metal surfaces. A word may be given to the camera itself, which was built at home, and is another example of easily made apparatus. It is of half-plate size, of very long bellows extension, and carried on two pieces of nickel-plated bicycle tubing. The door at the side can be opened to focus on a piece of card placed in the camera back. Stands for the micro- 
scope and lamp can be similarly made for this optical bench, but in the illustration Zeiss' triangular bar, carrying cooling trough, Köhler condenser, and mirror, on saddle stands, is used instead, and placed parallel to the microscope. A convergent beam of light is projected by condensers and mirror into a nose-piece vertical illuminator, and reflected thence by the cover-glass through the objective on to the prepared surface of the metal. The microscope is the Edinburgh Student's "H," and has not got a focussing stage. The substage is therefore made use of for carrying and focussing the metal. A tube is screwed in the position usually occupied by the condenser; it is filled with plasticine and the metal imbedded in this, care being taken that its prepared surface is horizontal by pressing it against a slide held across the microscope stage. This method of holding the metal has the advantage that only one surface of the latter has to be prepared, whereas if it is laid on a stage two surfaces have to be polished truly parallel. The condensers are adjusted to give critical or nearly critical light on the metal surface, which is brought into focus by means of the substage rack and pinion, and finally by the ordinary fine adjustment. The two photographs of Spiegeleisen given on Plate 28 were taken in this way, and the particulars given there will show the exposures, etc., required. The optical bench of this camera is as a rule fixed in line with that of the microscope, and is only moved to the side for such special purposes as metallography.

Another device whereby an ordinary microscope, fitted with vertical illuminator, can be used to photograph an object laid upon its stage is shown in diagram at Fig. 66 . A reflecting prism is attached to the nose-piece and directs a parallel beam of light into the illuminator however much the microscope tube is moved during focussing. 



\section{Micro-metallography.}

A Spiegeleisen $\times 45$

B Diтto $\times 500$

A

Zeiss Micro-Planar, $25 \mathrm{~mm}$.

Ocular

Illuminator

Illuminant

Aux. Condenser

Plate

Exposure

None.

B

Zeiss $16 \mathrm{~mm}$. Apo.

Zeiss Comp. $\times 12$.

Cover-glass reflector.

Liliput 4 amp. arc.

Biconvex lens, convergent beam, directed on to cover-glass reflector.

The specimen was polished, and etched with saturated solution of picric acid in absolute alcohol to exhibit its structure. Spiegeleisen is a highly crystalline variety of white cast-iron containing manganese.

Photographed with the apparatus of Fig. 65. using a cover-glass reflector. The aperture of the condenser restricted by an iris diaphragm to prevent haze when using the higher power objective. 


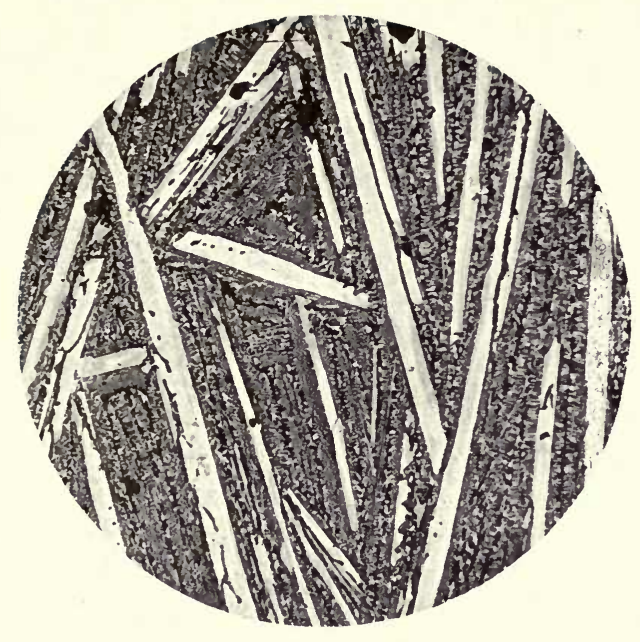

A

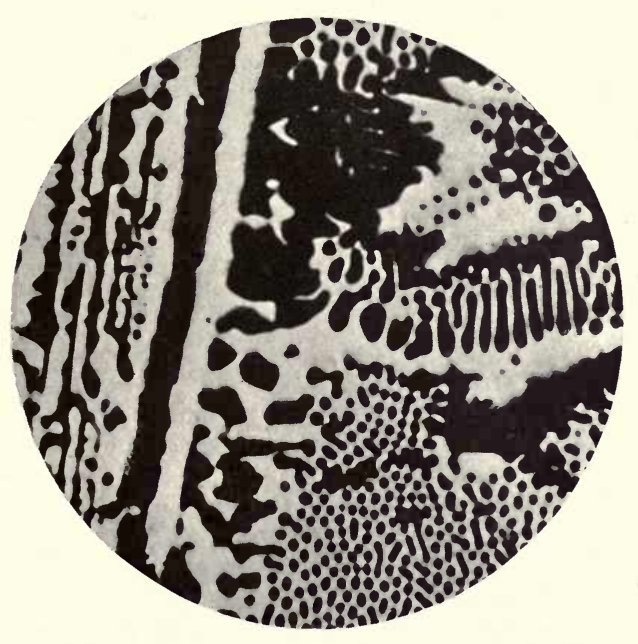

B 



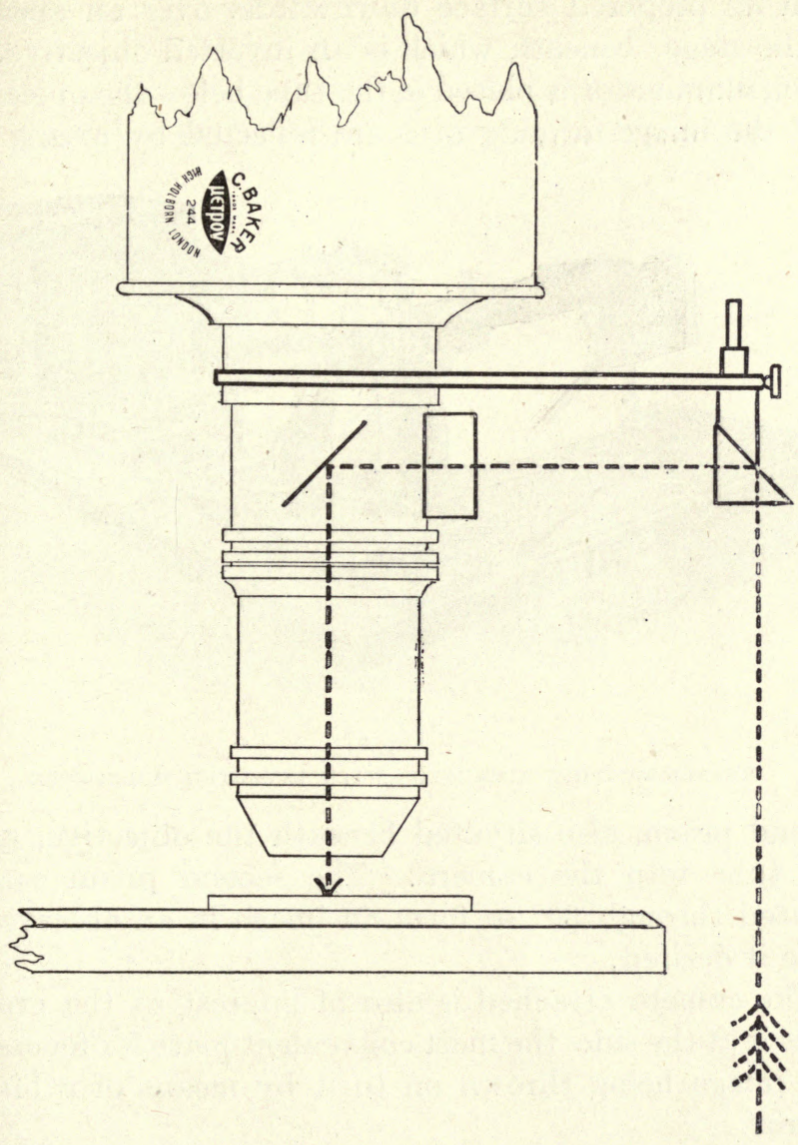

Fig. 66

PRISM ATTACHMENT FOR VERTICAL ILLUMINATOR

Micro-metallographs.-One or two firms (Leitz and Reichert) make special outfits on an entirely different design from the ordinary microscope and camera, to which they give the name of Micro-metallographs. That made 
by Reichert is shown at Fig. 67. The piece of metal is laid with its prepared surface downwards, over an aperture in the stage, beneath which is an inverted objective. A prism illuminator is placed in the tube below the objective, and the image-forming rays are reflected by means of a

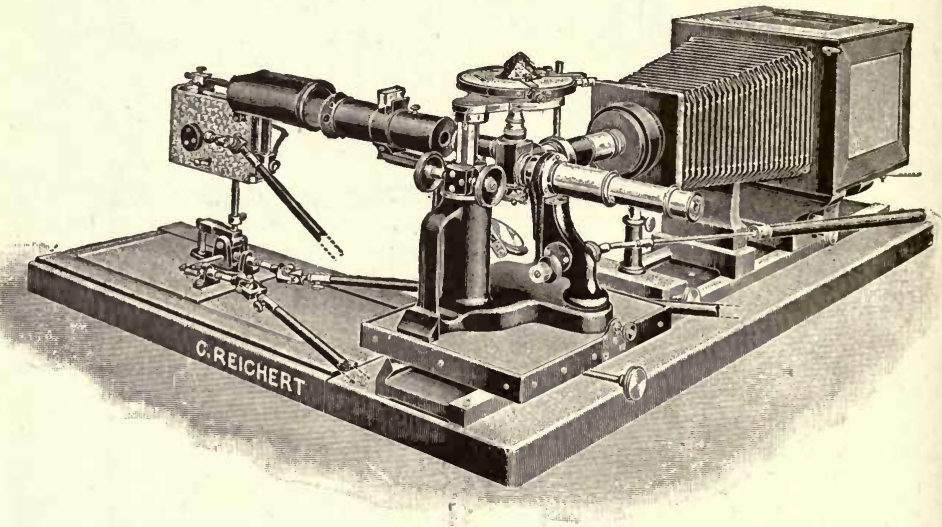

Fig. 67

METALLOGRAPIIC APPARATUS WITH INVERTED MICROSCOPE

second prism, also situated beneath the objective, along the tube into the camera. The second prism can be rotated through $90^{\circ}$ to form an image in an observation tube if desired.

The camera attached is also of interest as the ground glass is at the side, the most convenient place for focussing, the image being thrown on to it by means of a hinged mirror. 



\section{Micro-metallography.}

A Mild Steel $\times 50$ (carbon 0.25 per cent.).

Specimen polished, and etched with saturated solution of picric acid in alcohol, shows sorbitic pearlite with ferrite meshing.

B Monel Metal (copper nickel alloy) × 150 (33 per cent. copper, 64 per cent. nickel).

Etched by an electrolytic method, the ordinary methods being unsuitable. The alloy was connected to the anode in dilute sulphuric acid.

Vertical camera used, and cover glass nose-piece illuminator.

Preparation of steel surface (Stead's method).-The surface is first levelled by rubbing down by hand on a smooth file, the latter being laid flat on a table. Then on a fairly smooth English emery cloth, next on two or three French emery papers of increasing fineness, and finally polished on a revolving block over which is stretched black cloth impregnated with diamantine powder. 


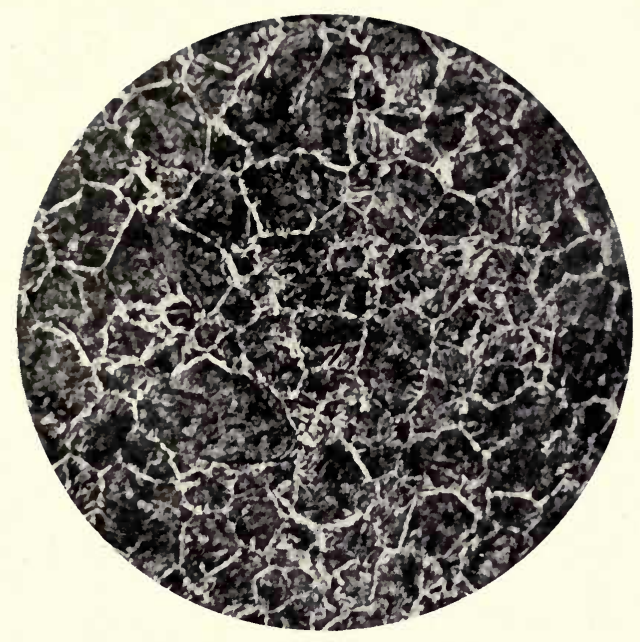

A

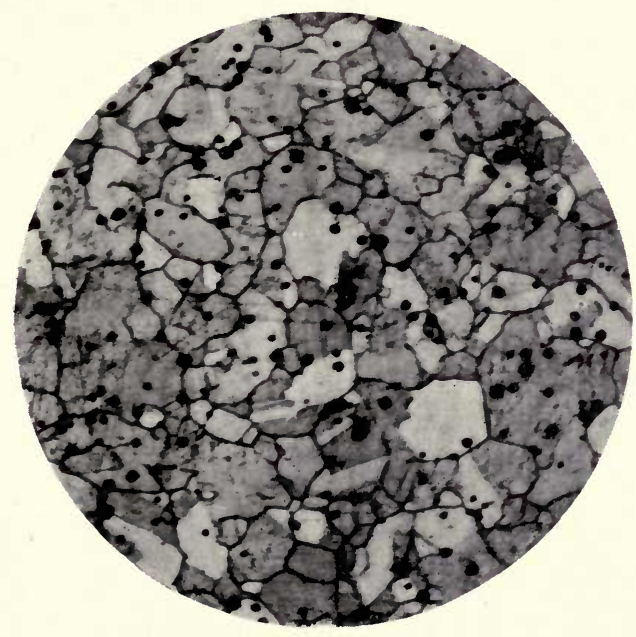

B 



\section{CHAPTER XIII}

\section{COLOUR PHOTOMICROGRAPHY}

THE possibilities of an exact reproduction of the appearance of a microscopic object have been immensely in. creased by the application of colour photography to photomicrography. A monochrome rendering of the majority of preparations is quite adequate for most purposes, but the great use made of staining processes and the advantages offered by these as a means of differentiation, owing to the selective action of both animal and vegetable tissues for various stains, are sufficient to call attention to the desirability of sometimes photographing the object in its actual colours; and blackand-white prints of naturally coloured objects can never be quite so satisfactory as pictures in which the original colours are faithfully reproduced.

The colour processes at present most in use result in the production of transparencies, which, however satisfactory for hand examination or projection, are inconvenient for storage. Efforts are being made with a considerable amount of success to perfect methods of reproduction on paper analogous to ordinary printing processes, but so far they are not of great use for photomicrography. Loss of detail is particularly noticeable in many of the prints so made, and any method, to be suitable for photomicrography, must give prints equalling in detail the original negative or transparency.

Reproductions of transparencies made by the Autochrome, Dufay, Paget, and Three-Colour processes are 
given as a frontispiece and on Plates 3, 10, 18, 22, 30 and 37.

The processes mentioned may be classified according to the method of application of the light filter into "Screen-plate" and "Three-Colour" processes. Both ultimately depend on analysis of the image-forming rays by their passage through three light filters-blue-violet, green, and red - each one of which absorbs the colour complementary to it, and transmits only a definite portion of the image-forming rays to give a negative on a panchromatic plate corresponding to one of the primary colours which make up the object. They differ fundamentally in the methods of employing the screens, and the process by which the coloured positive is built up, in one case, from three negatives taken independently through the three filters, and in the other, from one negative taken through an ingenious combination of the three filters on one plate. In the latter case the single negative combines in itself the three elementary images intimately blended.

It is obvious that three screens which will divide daylight into three components, so accurately balanced that a coloured positive can be obtained by suitable methods from the three negatives, will not do for artificial light relatively poor in blue rays, unless a variation can be made in the three exposures which will compensate for the incorrect colour analysis. If this is not possible the resulting negative formed by the blue rays would be relatively too weak by an amount depending on the deficiency of blue rays in the artificial light, and the positive would consequently be quite false to the original. This difficulty is nearly always encountered in photomicrography, as daylight is not often used, and the methods for overcoming it are detailed below in connection with the description of both processes.

Three-Colour Processes.-The analysis of the imageforming rays, and the subsequent synthesis of the coloured 
picture, are most simply explained by reference to the Sanger-Shepherd three-colour process. Three negatives are taken on panchromatic plates, one through each of three screens of the primary colours, blue-violet, green, and red. Sanger-Shepherd, and Wratten and Wainwright include filters suitably adjusted for their panchromatic plates in their sets of photomicrographic screens, and they are used in the way previously described for any other colour filter. Three negatives are taken, one after another, focussing carefully with each screen. Apochromatic objectives and compensating eyepieces are naturally most useful here, as any considerable error in the colour correction might produce images of unequal size on the plate; but good-class achromats are quite satisfactory. No other special apparatus is necessary, although a triple-repeating back to hold the three plates is a convenience.

The exposures necessary depend on the plate and the screens in conjunction with the illuminant used. In the case of Sanger-Shepherd tricolour screens and colour plates the exposures with daylight are the same through each screen.

With Wratten and Wainwright's screens in conjunction with their " $M$ " plate, the three exposures are in the following ratios for various illuminants.

\begin{tabular}{l|c|c|c|c|c}
\hline & $\begin{array}{c}\text { Daylight } \\
\text { or arc. }\end{array}$ & Nernst. & $\begin{array}{c}\text { Incandes- } \\
\text { cent gas. }\end{array}$ & Oil. & Acetylene. \\
\cline { 1 - 3 } & 1 & 1 & 1 & 1 & 1 \\
A. Red & 2 & 2 & 2 & 4 & $2 \frac{2}{5}$ \\
B. Green & 2 & $2 \frac{2}{3}$ & 2 & $8 \frac{1}{3}$ & $2 \frac{2}{5}$ \\
C. Blue & 2 & & & \\
\hline
\end{tabular}

The exposures must be carefully calculated in accordance with these factors, or any variant from them given in the plate box, and the plates should be from one batch to ensure their being of the same speed. This variation 
of the exposures through the three screens is all the compensation needed for the different constitution of the light from various sources. Mark the plates A, B, C with pencil on the corner of the film before development to correspond with the particular taking screen used.

To ensure equality of density in all three negatives the three plates must be developed at the same time in the same dish after accurately timed exposures. Development must be carried out in darkness for the proper time, depending on the developer and temperature; suitable times are usually given in the plate box for a definite developer. The light given by the safelight, allowable with the panchromatic plates, is not sufficient to enable a correct judgment of the density to be made by visual examination.

Three positive transparencies are made on bichromated gelatine on a celluloid base from the three negatives. Special celluloid films coated with gelatine and silver bromide emulsion can be purchased from Sanger-Shepherd. They may be obtained ready sensitised, but it is better to sensitise the films for oneself. To do this the film is placed in a solution of ammonium bichromate (p. 273) and bathed for about five minutes, and afterwards dried in the dark.

The films are then placed with the shiny celluloid surface (not the sensitive side) next the negative and printed like P.O.P., using an actinometer to get correct depth of printing. A suitable guide to the time of printing is obtained by printing a piece of P.O.P. under a negative of similar density side by side with the colour negative, and removing the film when the P.O.P. is printed to a depth somewhat lighter than is usual for that type of print. Each film must be marked A, B, or C to correspond with the negative from which it was printed.

After printing, the films are developed by immersion in warm water (about $100^{\circ} \mathrm{F}$.) until the parts of the 
gelatine unacted upon by light, and still soluble, have been dissolved away. The presence of silver salts in the emulsion renders development easy, as all details of the image can be distinctly seen. To get rid of all traces of silver salt in the emulsion the films are placed in hypo until the creamy appearance entirely disappears and three perfectly transparent positives are obtained. They are then washed thoroughly and dyed, as follows, in solutions complementary in colour to their respective taking screens.

Film A from the red negative is dyed in a dilute acid solution of fast green.

Film B from the green negative is dyed in a solution of erythrosine, and afterwards bathed in a $5 \%$ solution of copper sulphate, which fixes the dye to a certain extent and renders the colour less liable to fade on exposure to light.

Film $\mathrm{C}$ from the blue-violet negative is dyed in a dilute acidulated solution of napthol yellow.

After dyeing, and before drying, the three films are placed one above another in correct superposition to see if the colours are of the right tint, as the correct rendering of the colours of the original depends on the correct tint being obtained in each of the coloured transparencies. Any deficiency or excess of one or other of the colours can be made good by further dyeing, or longer soaking of the particular film in water to which a few drops of acetic acid have been added. When a correct appearance has been arrived at the three positives are dried. The red one is then carefully superimposed on the blue one, and the two fastened together. Finally the yellow print is adjusted on the others, and all three bound together between two cover-glasses.

In dyeing the positives it is advisable to use very dilute solutions of the dyes, and to leave the prints in them for a considerable time, as this tends to give more vigorous 
prints with better half-tones. (Formulæ are given on p. 273.)

The blue-green transparency may be made on an ordinary lantern plate in the usual way for black tones, and toning in one of the Leto toners (the blue one), or by immersing it in a bath of Potassium ferri-cyanide and Ferric ammonium citrate (p. 273). It should be borne in mind that this procedure intensifies the image, so that the black transparency should not be quite so dense as is required in the final blue image.

The mouse's head on Plate 30 is an example of the three-colour process, and the particulars given with it will give some idea of the exposures and other working data.

Two-Colour Process.-It is not so essential that the colours of stained sections should be as accurately reproduced in a photograph as the colours of a naturally coloured object. The chief object of differential staining is the emphasis and discrimination of the salient features of the section. The various stains are of use principally on account of their selective properties, and the depth of tint produced can be varied at will. This gives great latitude in colour photomicrography, and renders unnecessary a very nice adjustment of the dyed positives in the majority of eases, and even permits of the use of a very simple "Two-colour process." Plant or animal sections, for instance, which have been double-stained in approximately complementary colours, say red and green, or blue and yellow, can be satisfactorily photographed through two screens made with the identical dyes used for staining the section, by soaking cleared gelatine plates in dilute solutions, as described on page $\mathbf{1 5 6 .}$

To photograph a green and red section, for instance, take two negatives, one through the green and the other through the red screen. Make positives by means of the bichromated gelatine process, and stain that from the 



\section{Direct Colour Photomicrograph.}

Three-colour process.

Transverse Segtion Head of newly-born Mouse $\times 8$

Objective ... ... Zeiss Planar. $50 \mathrm{~mm}$.

Condenser ... Watson's Universal Achromatic, back combination.

Illuminant ... Incandescent gas.

Aux. Condenser Zeiss Köhler condenser, parallel light.

Plates $\quad . . . \quad$... Wratten's “M” Panchromatic.

Three negatives were taken through Wratten's tricolour filters A (scarlet), B (green), C (blue-violet).

Exposures-A, 3 seconds; B and C, 6 seconds each.

The positive from which the reproduction was made is a transparency composed of three Sanger-Shepherd celluloid films, dyed as described on page 206 .

Preparation stained in bulk with borax carmine, counterstained in aniline blue and picric acid. 


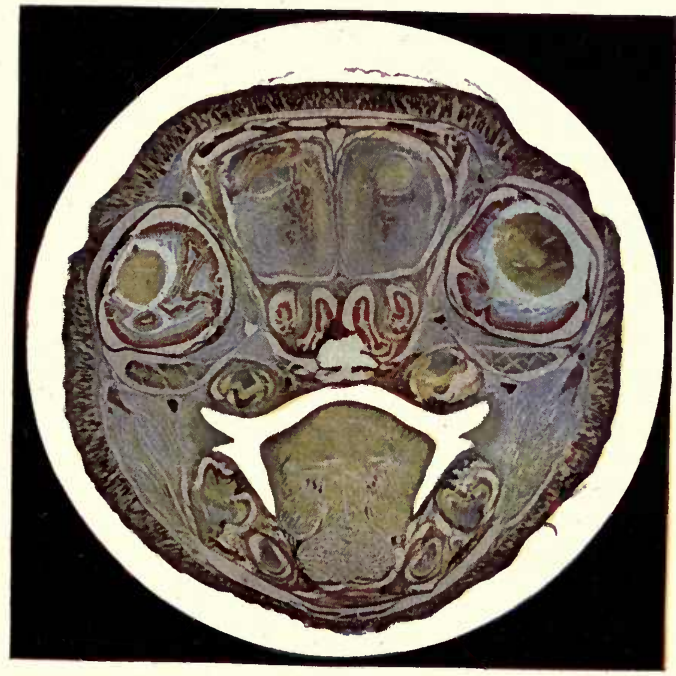


$\infty$ 
red negative with the green dye, and that from the green negative in the red dye, binding them together as described in the three-colour process.

If a section is stained blue and yellow, or blue and green, satisfactory negatives may be obtained on orthochromatic plates ; it is only when an orange or xul colour has to be reproduced that it is absolutely necessary to use panchromatic plates. Generally, however, the reproductions only approximate to the colours of the original, but for the purposes for which the process is suitable this is immaterial.

Screen-Plate Processes.-The Autochrome, Dufay, and Paget colour plates are typical of the "Screen-plate" processes, which differ from the "Three-colour" in that only one negative is taken. There are other processes besides these, but only the three of which examples are given are here described. Instead of the three-colour filters previously described, only one is used, consisting of minute elements of red-orange, green, and blue-violet entirely covering the plate. The elements are so small that at the normal distance of distinct vision the eye can hardly detect them, and the screen should appear of a neutral grey colour. They consist in the Autochrome plate of irregularly scattered grains of starch suitably dyed, while the screen of the Paget and Dufay plates is made by dyeing a collodion or gelatine film in such a way that it is covered with a mosaic of regularly arranged square or rhombic elements.

The colours used in the screen are not the same as those of the three-colour screens. Each element fulfils the double purpose of a taking and viewing screen, and consequently they are mutually adjusted for that purpose. The exposure through each element is the same, and no allowance for a variation in the nature of the illuminant can be made in that way. A yellow lint filter properly adjusted to the screen and plate is an essential part of the 
colour system, and it offers a means of adapting the plate to any source of light. Paler yellow screens, or sometimes even a green one, are used with artificial illuminants to compensate for the smaller proportion of blue rays or over-abundance of red rays. Suitable screens can be obtained in several cases from the plate maker, and accurate colour-rendering cannot be obtained unless a correctly adjusted one is used. The $\mathrm{K}_{1}$ and $\mathrm{K}_{2}$ screens of Wratten and Wainwright are also useful for this purpose.

"Single" Processes.-Autochrome, Dufay, or Paget. The glass support is coated with the colour screen, and a panchromatic emulsion then spread on top of that, the whole forming a single plate. When loading the dark slide, in darkness or by one of the special safelights, the plate must be placed with the emulsion surface to the back, so that exposure is made through the glass and colour screen. A piece of card is placed behind the film to protect its delicate surface from abrasion. To focus accurately the ground glass is reversed, and used with the ground side away from the microscope in register with the film side of the plate. The result of exposure and development is the formation of a negative consisting of dots corresponding to the different coloured elements of the screen. Red light, passing through the red elements only, produces (after development) a black spot under each, and, if the negative so produced be held up to the light, these spots prevent light passing through the red parts, and the plate appears a complementary blue-green from a blending of the light passing through the blue and green elements.

A transparency showing the colours of the original is obtained by removing the black dots of the negative, and replacing the clear parts of the negative by black. Thus, in the example given, red light only can pass through the plate after the removal of the black dots under the red 
elements and the subsequent blocking out of the green and blue. This process is called "Reversal," and is a conversion of the negative to a positive.

The whole process of development and reversal is very quickly carried out, the principle of the reversal being an oxidation of the black parts of the negative by potassium permanganate or bichromate, and a subsequent darkening of the parts not hitherto developed, by immersion of the plate in the original developer in bright light. The plate is finally fixed in hypo (although this is stated by Messrs. Lumière to be unnecessary), washed, dried, and varnished. What is judged to be the right quantity. of varnish is poured on to one corner of the plate, and is caused to flow evenly over the entire surface by judicious tilting of the plate towards each corner in turns. Any excess is run back into the bottle.

General formulæ suitable for any screen plate are given in the appendix (pp. 273-4) with an outline of the process, but each maker advises some method particularly suited to his plates; or some special developer, such as Quinomet. It is unnecessary to give these, as the makers supply the details, but advantage undoubtedly arises from carefully following the instructions accompanying the plates when they differ from the general formulæ given.

A true colour-rendering depends very largely on correct exposure; an idea of what is necessary can be gathered from the descriptions accompanying the colour plates. Over-exposure gives a too dense negative, and consequently on reversal a thin positive, very poor in colour. Too short exposure leads to a dense positive, through which the colours can only with difficulty be seen. The Howard Farmer reducer and silver or mercury intensifiers can be used to improve defective positives.

"Duplicating" Process.-A drawback, of the single process is that each plate embodies a screen, which is an expensive item, and the Thames Plate Co. overcame this 
by making the screen on a separate piece of glass, so that it is still of use even if the plate is spoilt, and exposing a panchromatic plate in the camera behind the screen film to film. The Thames plate is not now on the market, but the Paget Prize Plate Co. make a screen of this sort and supply it together with suitable plates.

The negative is made as in the single method, but it is fixed, and not reversed. From it clear black positives are made on lantern plates; metol-quinol is a good developer for these, and they are bound up in register face to face with a viewing screen similar to that used for taking the photograph. The process of binding up in exact register requires a considerable amount of care. Some practice, however, makes it fairly rapid and easy. The first appearance when the screen and positive are put together is somewhat like a Scots plaid,. and by moving the two plates slightly the size of the pattern is varied. When it is as large as possible the plates are gripped with 'a bull-dog clamp, and a very slight movement brings them into exact register, when correct colourrendering is seen. The plates are then bound up together with lantern-slide binding. It is very easy to get a reproduction in colours complementary to the original by a slight error in register, and care must be taken that the adjustment is such that the colours are correctly rendered.

Comparison of the Colour Processes.-The best results, the most brilliant colours, and clearest slides with sharpest definition are undoubtedly produced by the three-colour process, but it is at the same time the most tedious to work. Any number of slides can be made from one set of three negatives.

The single-screen process is the simplest, but has the drawback that only one positive is obtainable from each exposure. The duplicating-screen process enables as many positives to be made as may be desired from one negative, and in that respect is very superior to the single process. 
All the screen plates have the disadvantage of a grain of the coloured elements over the surface, which prevents the reproduction of fine detail, and may on close inspection or projection be troublesome.

The Dufay and Paget plates give positives that are considerably more transparent than the Autochrome, and can be projected more easily in a lantern. It is also possible to wash the emulsion from spoilt plates of these makes, leaving a serviceable screen for the duplicating process.

The screen plates require rather long exposures, which in certain cases is a drawback. Messrs. Lumière now make a special sensitising bath by which the speed of their plates can be increased 4 or 5 times, and supply a screen suitable for use with the extra-sensitised plates. 


\section{CHAPTER XIV}

\section{PHOTOGRAPHIC OPERATIONS}

Plates.-There is no need to describe in detail the ordinary routine of photographic processes, but the points of chief importance in photomicrography require more than passing mention, particularly on account of the great use that is made of colour sensitive plates. The advantages of panchromatic plates have been mentioned in the chapter devoted to colour screens, and they would always be used were it not for their cost and the diffculties of manipulation-though these are really not great after a little experience-due to the necessity of developing them either in darkness or in a very dull green light. For these reasons orthochromatic plates are much more frequently used than panchromatic, and for many purposes their lack of sensitiveness to the orange and red rays is of no great moment. Non-colour sensitive plates are as a rule not nearly so satisfactory for the reasons which have already been given.

Panchromatic Plates.-There are not many makers of panchromatic plates, but the following are well-known to us. Wratten and Wainwright's "Panchromatic " and "M" plates, the latter specially made for photomicrography with a fine grain. Ilford's Bathed plates, Paget's "Process Panchromatic" and "Hydra Panchromatic," Gem "Tricol," and Sanger-Shepherd Colour plates. Some workers prefer to sensitise ordinary plates to yellow and red by bathing them in certain dyes as required, both on account of the expense of panchromatic plates and their liability to lose their colour sensitiveness in time. It is well to select fine-grained ordinary or medium plates for this purpose, and the 
process together with a formula for the dyeing bath is given in the appendix, page 270.

Orthochromatic Plates.-Practically all manufacturers make orthochromatic plates in two or three speeds, and some with different degrees of colour sensitiveness. Some brands are dyed in such a way as to be proportionally less sensitive to blue, and work as if they were orthochromatic plates with a pale yellow screen. They are known under such names as Non-Filter, Anti-Screen, Non-Screen, etc., and can be well recommended when the influence of a pale yellow screen would not be disturbing. Plate Speeds.-Plates are roughly divided, according to their speed, into Process, Ordinary, Medium, Rapid, and Ultra-Rapid, different makers having different names for these groups. As a rule the speeds marked on the box are only a rough guide to the exposure required. They apply to exposures in daylight, and the exposures in artificial lights, more particularly when using a colour screen, depend on the colour sensitiveness of the plate sometimes to a greater extent than on their speed, and even a process panchromatic plate might require less exposure for a certain subject than an ultra-rapid noncolour sensitive plate. Rapidity is, as a rule, gained at the expense of fineness of grain, latitude, and resistance to fogging during development. The rapid plates also tend to give less contrast and density. For these reasons the slower the plate that can be used the better. Process or ordinary plates are usually quite fast enough for arc or limelight, but with less powerful illuminants medium or rapid plates must generally be used, as it is most desirable that exposures be cut down to avoid trouble due to vibration and heat. Plate makers are constantly extending the desirable characteristics of slow plates, such as latitude, ease of development, freedom from fog, sharpness of image, etc., to the more rapid kinds, and very few of the rapid brands will be found to offer any difficulties. 
Resolving Power of Plates.-Resolution depends mainly on the optical arrangements of the photomicrographic apparatus, but to a certain extent the nature of the plate limits the fineness of detail that can be sharply reproduced. The transition also from dark to light in the photographic reproduction of lines and points on a light ground, or bright detail on a dark ground, is more or less gradual and indefinite, and causes very minute, closely packed lines, etc., to merge one into another. Dr. C. E. K. Mees examined this question and found that the "Resolving Power of Plates" increases as the size of the grains in the emulsion diminishes. An extra-rapid plate with grains .0015 to $.004 \mathrm{~mm}$. in diameter could just separate lines $.030 \mathrm{~mm}$. apart, while a process plate, in which the grains are .0010 to $.0015 \mathrm{~mm}$. in diameter, resolved lines $.018 \mathrm{~mm}$. apart. The increase in resolving power does not go much beyond this, as smaller grains set up diffractions which act in the opposite direction.

More recently,Dr.E.Goldberghasinvestigated the spreading of the light action from the lines and points of detail into the surrounding parts of the plate, and in a paper communicated to the Royal Photographic Society stated that the "Sharpness of a Plate" depended on the ratio of the "Development factor" to the "Turbidity factor" of the plate. The first of these factors expresses the gradations produced by the plate, the second is the ratio of the light scattered in the film to thatfalling on it. The greatest sharpness of image is given by the plate which has the smallest turbidity factor and the greatest development factor.

The Lippmann grainless plates have a low development factor-that is, are very soft-working or give flat gradations, but their turbidity factor is so exceedingly small that the quotient of the first factor by the second is so high as to determine a very sharp image.

Process plates which are distinguished for their capacity to give sharp reproductions are very hard- 
working, and have a comparatively small turbidity factor, and the ratio of the two factors is high. Rapid plates, as a rule, show a greater spreading of the light action consequent on a lower development factor and greater turbidity factor. The spreading does not, however, depend on the size of the grain of the plate; some fine-grained plates give a greater spreading than others with a coarser grain.

The sharpness with which detail is reproduced also depends on exposure. With rapid exposures there is less spreading than with more prolonged, but a regular alternation of black and white elements, for which a given short exposure would be equally good over the whole surface, is not often encountered in photomicrography. More generally it is necessary to represent fine detail against a light background in one part of the preparation, and at the same time detail in another part which is thick or coloured. An exposure, small enough to prevent spreading in the former, would probably give no sign of detail in the dense part. And a correct exposure for the latter would hopelessly blur, or entirely obliterate, the fine detail in the thin part or against the background by the encroachment of the high lights over it.

Halation.-This term is generally understood to cover the obliteration of fine detail in this way, and the spreading of the effects of light action over the adjacent shadow areas. It also refers to a second action on the back of the film, caused by light which reaches the back of the plate, where it is reflected at the glass and air surface back again through the glass to the under side of the film, producing there a secondary image on either side of the primary one.

Fig. 68 includes several photomicrographs of the nearly closed slit of a spectroscope taken by a $1 \frac{1}{2}$ " achromatic objective on one plate, and originally published in the Amateur Photographer. Seven exposures were given in the ratio $1: 2: 4: 8: 16: 32: 64$, and the 
plate moved along a little after each. Half the plate was coated on the back with a thin film of caramel. The print shows the spreading of light described in the last section as limiting the resolving power of the plate, and indicates that it is least with a short exposure, and gradually increases. The secondary images, caused by
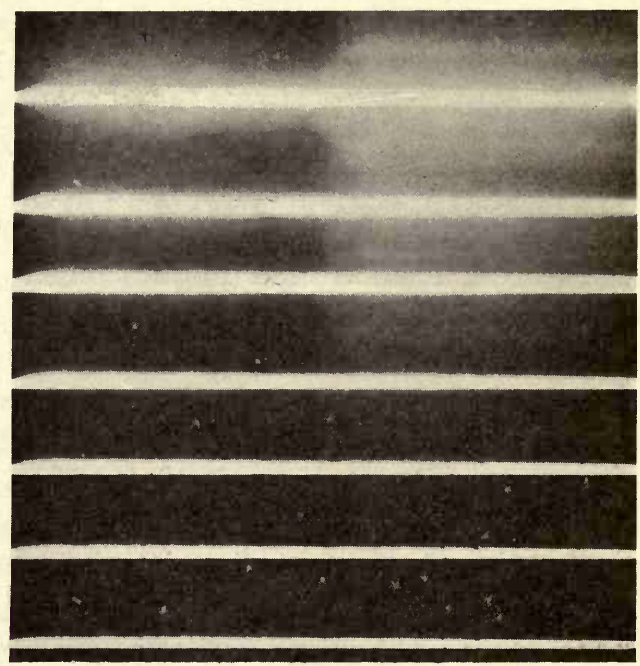

Fig. 68

HALATION

light which has passed from the brightly lighted lines on the film to the back of the plate and was there totally reflected, are seen to occur only when gross overexposure has been given. As has been pointed out, over-exposure is liable to occur in parts of a photomicrograph, and it is advisable to take such preventive measures as will avoid the reflections. They are not apparent on the backed half of the negative, showing that the coating of caramel is an effective preventive, but it is obvious on examining the figure that the backing 
does not prevent the spreading of light in the film, but it does cause a decided improvement in the general brightness of the negative.

Films and Backed Plates.-The thinner the glass the nearer the secondary images approximate to the central one, and on films they are practically coincident. Although films have this great advantage over plates and are much more convenient for storage, they are not so satisfactory on the whole, and most workers find it easier to get good results on plates.

Backed plates should always be used in order to avoid halation as much as possible. The backing takes several forms. A soluble coating of caramel is very satisfactory and need not be removed before development, as it dissolves in the solution; the developing solution becomes black, but if only used for one or two plates no harm results. Some makers mix insoluble black or red pigments in the backing material, in which case it should be removed with a damp sponge before development, otherwise pieces may float on to the surface of the film, from whence they are difficult to dislodge. It is usual to buy plates ready backed, but the glass side of unbacked plates can easily be coated with caramel, which is specially sold for that purpose. If too thick to spread evenly the syrup should be thinned somewhat with alcohol, and rubbed on the back of the plate with a glass rod over which a piece of rubber tubing has been passed. The rod is conveniently shaped like a $\mathrm{D}$, the tubing covering the straight side. A very thin layer of backing only is required, and this soon dries; but if the plate is wanted immediately the backing should be covered with a piece of paper before placing in the dark slide. It is very difficult to back panchromatic plates on account of the necessity for working in the dark, and they should always be bought ready backed. An anti-halation film between emulsion and glass is adopted in the Chromo- 
Isolar and Paragon plates. It dissolves out during fixation and forms a very clean backing.

Developers. - There is a very long list of developers from which to choose, and their characteristics vary to some extent. There are quick- and slow-acting developers, developers that tend to give a yellow negative, and others that give a grey-black image. Some are greatly affected by the temperature of development, while others are not so sensitive in this respect, and so on. A few reliable formulæ are given in the appendix, but the inferiority of others is in no way implied. Suitable formulæ for most developing agents are to be found in such useful books of reference as the British Journal Photographic Almanac and The Photographic Annual, and practically any of them, if rightly used, will give first-rate negatives for photomicrographs. It is necessary, however, to know the characteristics of whatever developer is selected, and to use it with judgment in order to obtain a negative of the desired quality. Some developers tend to give negatives with great contrasts, while others more readily give softer results, but an equally good negative could be obtained with either sort if used properly.

Developers do not, as a rule, keep long in dilute solutions, and for intermittent use are most conveniently obtained in tabloid form and dissolved as required, or kept in concentrated solutions and diluted for use. Of the latter type there are many on the market containing, as a rule, para-amidophenol and a caustic alkali, such as Rodinal, Azol, Victol, Certinal, ete., and they are particularly useful, serving either for plates, papers, or lantern slides. Tabloid Rytol is a similar universal developer. If development is frequent it is more economical to make up one's own solutions from chemicals bought in suitable quantities.

Pyro Soda has the advantage of being among the cheapest as well as the best developers, but it has the 
disadvantage that the pyro does not keep well in solution and that the solution stains the hands. A good plan is to prepare the No. 2 solution as stock, and to add weighed quantities of dry pyrogallol when required, but, if sublimed pyro in powder is used, it is almost equally accurate to measure it. For this purpose a teaspoon may be used, the weight of the contents of which is known. This developer is not suitable for papers on account of its staining property. A yellow-stained negative is often, on the other hand, better than a clear black one. The very non-actinic image gives a more contrasty print.

Metol-Quinol is an exceedingly fine developer, acting more quickly than pyro soda, and useful for papers as well as plates. It has, however, the great drawback that metol may cause a painful skin affection, and for that reason the fingers should be dipped into it as little as possible, papers being manipulated by means of forceps. A rinse in a very dilute hydrochloric or acetic acid solution is a preventive of the poisoning. The formula given is the concentrated solution of Mr. R. L. Boyd, and keeps good for a long time in a tightly corked bottle; it is diluted as required for use.

Hydroquinone or Quinol tends to give very harsh contrasts, and is selected when that is required, but the mixture with metol is generally preferable. The metol supplies detail and rapidity.

Potassium Bromide in Developers.-There is a growing tendency to the omission of potassium bromide in developers, but as it generally helps to give crispness to a negative and prevent fog it is well to include it for photomicrography, as bright contrasty negatives are required. One or two drops of a $10 \%$ solution is usually sufficient per oz. of developer.

Development of the Plate.-The aim of development is to produce a negative having sufficient contrast to show 
all detail clearly, and of the right density to print well. This can be assured, after correct exposure, by carefully watching the plate during development, when a good ruby light is allowable. It is difficult or impossible, however, to watch the course of development of panchromatic and, to a less extent, orthochromatic plates, and a mechanical or automatic means for controlling their development has been sought for and found in "Time development." This process is now very largely used, and can be relied upon to give results at least as good as, and generally better than the older methods.

Time Development.-Experiment has shown for a given brand of plates and the same developer used at a definite temperature, and within certain limits of exposure depending on the latitude of the plate, that :-

1. The same degree of contrast or gradation is always obtained by development for a given time.

2. The density attained in that time depends on the exposure given.

Most plate makers give the times at $55^{\circ}, 60^{\circ}$, and $65^{\circ}$ $\mathrm{F}$., for which it is necessary to immerse their plates in a given developer to get a good negative, and similar lists of the times required by well-known plates are given with most proprietary developers. Unless otherwise stated, these times will produce a negative of medium gradation suitable for landscapes, and must be increased by a quarter or a half to obtain the greater contrast desirable in a photomicrograph. Suitable development times are given with the developers in the appendix, but as different brands of plates behave in different ways, they may require lengthening or curtailment to produce the desired result with the particular plate used. One or two trials will determine the correct time in any given case.

The chief difficulty in time development is to keep the 
temperature of the solution constant. The simplest way, when the dark room is not too hot or cold, is to allow the developer and dishes to stand until they attain its temperature. Errors in the time of development, following correct exposure, are evidenced by a too great or too small degree of contrast in the resulting negative, and the time should be raduced or increased for future negatives.

Stand or Tank Development is a modification of time development, whereby half a dozen or a dozen plates are developed at once in a dilute solution of developer. The ordinary dish development is more economical when only a few plates are to be developed, but a tank is invaluable when many plates can be developed at once, saving time and rendering needless a long stay in the dark room. The plates are placed in a grooved holder in a covered box, and sufficient developer is poured in to cover them well, care being taken by judicious movement of the plates or of the box as a whole, at frequent intervals, to prevent air bubbles, and to keep the developer sufficiently shaken up. The solution is used very much more dilute than in a dish, and development is correspondingly prolonged, say from 15 to 30 minutes, according to developer and temperature. The only part of the process that need be carried on in the dark-room is that of filling the tank with plates and pouring on the developer. With many of the tanks on the market the latter operation, and also washing and fixing, may be done in daylight, a lighttight funnel being fitted to allow admission of solutions. But the slow pouring in of developer often leads to lines across the negative due to momentary high-water marks as the solution creeps up. Times for stand development are given with the suitable developers on page 269. Particular attention must be paid to keeping the temperature constant if time is to be relied on alone, and it is advisable to examine each plate before placing it in the fixing bath to en- 
sure correct development. There are many excellent tanks on the market, such as those of Griffin, Houghton, Kodak, and Watkins, but a covered enamelled iron box with grooves down each side to hold the plates may be used instead.

Factorial Development.-When it is possible to watch the development of a plate, the rapidity with which the image appears is a valuable indication of the correctness of the exposure given. Over-exposure is followed by a very quick appearance, while under-exposure determines a slow appearance with a hanging back of the dark parts of the object. Mr. Watkins has found that if the time required for the first appearance of the salient part of any image is noted, the best result in regard to density and contrast will be obtained if the plate is kept in the developer for a fixed multiple of that time; the multiple depending on the developer and type of negative required, and being independent of the temperature. Factors are given with each developer in the appendix; they are correct for the majority of photomicrographs, but may require to be increased or decreased at the judgment of the operator if a more or less contrasty negative than usual is desired. The method is a great favourite with many workers, but is not easily carried out with coloursensitive plates on account of the difficulty of seeing the first appearance of the image.

Fixing and Washing.-It is hardly necessary to insist that fixation be complete as indicated by the absence of any trace of white, such as is seen on looking at the back of imperfectly fixed plates. It is advisable to rinse plates after removal from developer and before placing in the fixing bath, particularly with pyro, otherwise the fixing bath rapidly becomes discoloured and may stain. To prevent any discolouration and stain an acid fixing bath is advantageous. Potassium meta-bisulphite is generally mixed with the hypo for this purpose. A clearing bath is useful for brightening negatives and 



\section{Pathological Sections.}

Section of a Cancer of the Womb.

Magnification ...

Objective ... ...

Ocular $\quad \ldots \quad \ldots$

Condenser

Illuminant $\quad$...

Screen $\quad \ldots \quad \ldots$

Plate $\quad \ldots \quad \ldots$

Exposure ... ...
100.

\section{A \\ A}

Swift $\frac{21}{3}$ Achromat.

Watson's Holos. 10.

Watson's Universal, front lens removed.
B

700 .

Zeiss $4 \mathrm{~mm}$. Apo.

Zeiss Comp. 12.

Watson's Universal.

N.A. 0.6 .

The sections show masses of growth cells arranged in alveolar formation with very little fibrous tissue between them. They are stained with hæmatoxylin and eosin.

For sections of portions from the same Cancer after treatment with Radium, see following plate. 


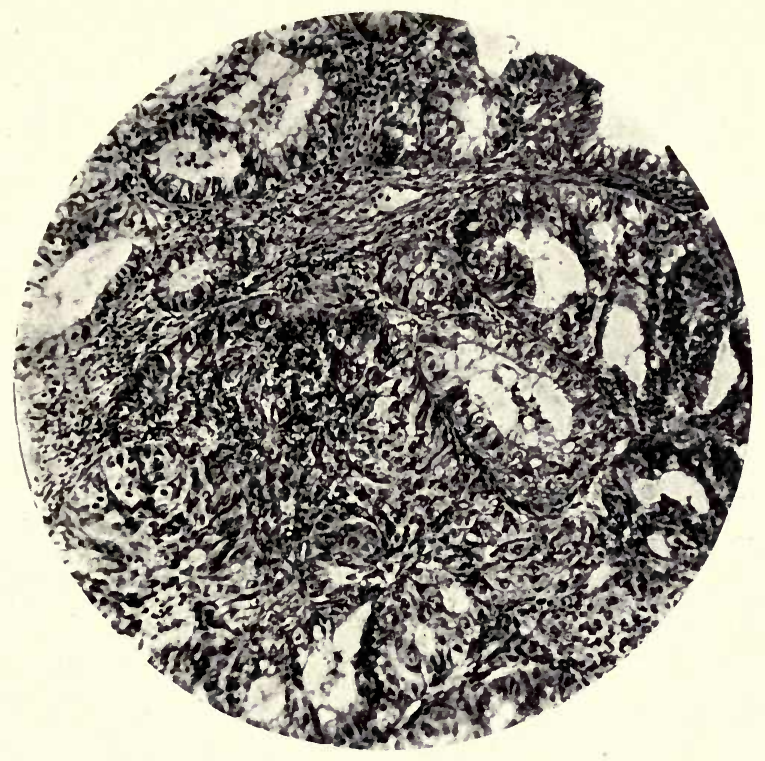

A

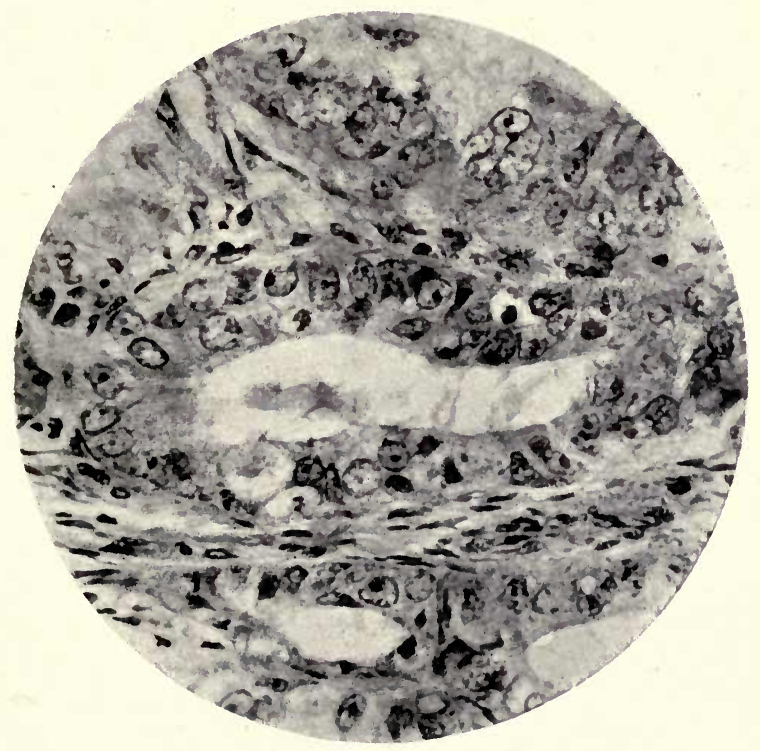






\section{Pathological Sections.}

Secisions of portions of the same Cancer after treatment with Radium. (Compare Plate 31.)

The photographic data are exactly the same as those given for A and B, Plate 31 .

The effect of the Radium treatment may be gathered from the changed appearance of the sections. Masses of fibrous tissue are visible, together with blood vessels with very much thickened walls, and a few patches of necrotic growth.

The four photomicrographs, cancer sections, are selected as an example of the use of photomicrography in pathology. The best general view of the section is obtained by using a $\frac{2}{3}$ " objective with a medium eye-piece giving a magnification of 100 times, but the structure of the cells cannot be readily made out unless an amplification of 500 to 700 times is employed. For this purpose a $\frac{1}{6}{ }^{\prime \prime}$ of low N.A. with a high eye-piece or, better, a long camera extension is most suitable.

Photos. H. L. H. from sections kindly

supplied by Dr. N. S. Finzi. 


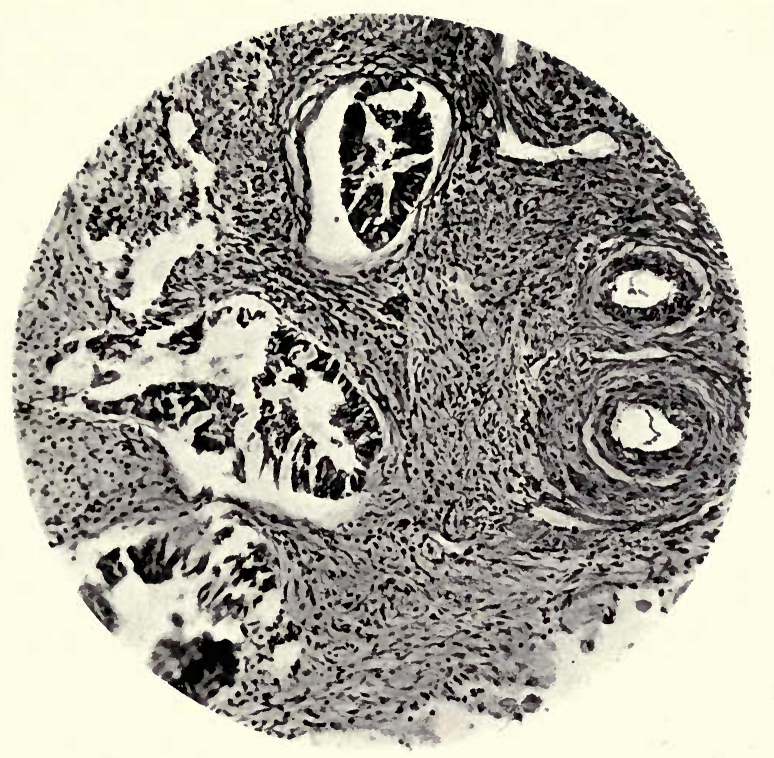

A

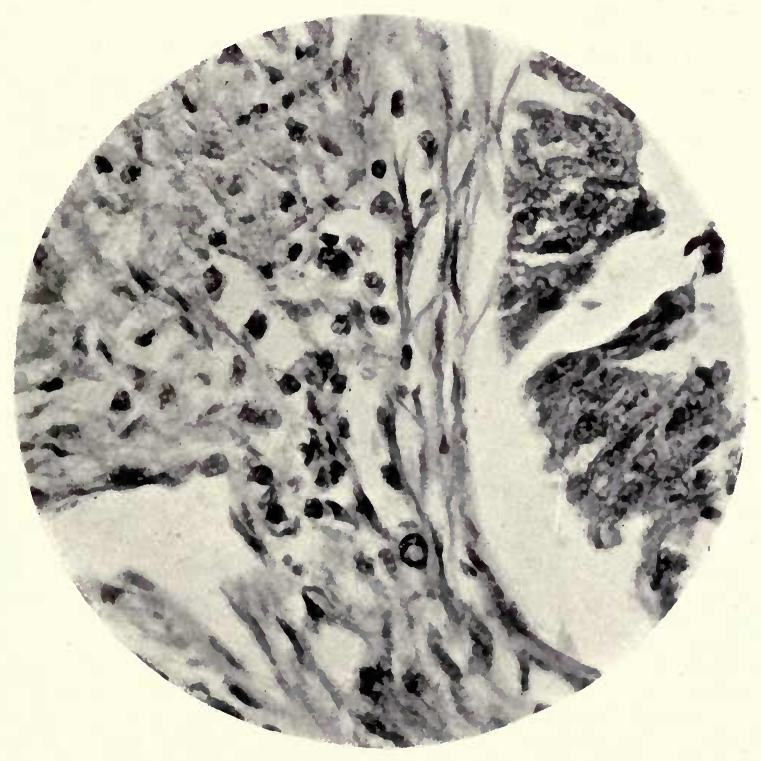

B 

prints before final washing; chrome alum and citric or hydrochloric acid are useful for this purpose. Washing after fixation must be complete : an hour in running water is sufficient if the water is continually removed from the bottom of the dish. Otherwise let the plates soak for five minutes in each of twelve changes of water.

Hypo Eliminators.-If it is desired to eliminate the hypo as rapidly as possible, the plates are immersed in several changes of a very dilute solution of potassium permanganate until no change in the pale, pink-coloured solution takes place; a short rinse in water is then all that is necessary.

Rapid Drying of Negatives.-To dry quickly, drain the plate and then immerse for a few minutes in alcohol; on withdrawal the plate will rapidly dry if placed in a warm place or in a current of air. Or harden in formalin and dry near a fire.

Hardening Negatives.-Negatives may be hardened if desired by immersion in formalin solution; they are then much less liable to damage. A varnish may also be carefully poured over the negative as a further protection.

Control by Exposure and Development.-A satisfactory printing density, revealing the details in both light and dark parts of the negative, is as necessary as proper contrast, and depends on exposure. If the latter is reasonably correct, time development will unfailingly produce good negatives, and most plates, especially the slower kinds, allow of considerable latitude on exposure. Correct exposure and development have so far been understood to mean such as will give a photomicrograph agreeing as closely as possible with the original, or possibly having a somewhat steeper gradation, as that is more often than not desirable in a photomicrograph. The degree of contrast shown between the various parts of an object must, however, be materially altered in many cases, either increased or decreased, to 
give an adequate representation. The use of colour screens for this purpose has been described in Chapter IX, and their effect depends ultimately, to a considerable extent, on exposure, but a great deal can be done in this direction by adjusting the exposure and development. Under-exposure, followed by a lengthened development, especially if a hard-working developer is used, will increase the contrast between light and dark parts of an object, but probably at the expense of detail. A longer exposure and shorter development will decrease the contrast, but give the shadow detail.

Errors in exposure can be detected in the negative by the following appearances, provided the plate has remained in the developer for the normal time and the errors are not too great.

Over-Exposure.-The contrasts of the negative are good, the detail is all there, but the negative is too dense to print easily. The tendency when developing an overexposed plate by inspection is to withdraw it too soon, or when the density appears correct. This leads to flat negatives, often, but erroneously, looked upon as the necessary result of over-exposure.

Under-Exposure.-The negative will be thin but of good contrast. A hard negative giving a " soot and whitewash" print lacking in detail may easily result from under-exposure, owing to the tendency to keep the plate in the developer long enough to force up detail in the shadows, by which time the lighter parts may be hopelessly dense and have lost all detail.

Improvement of Poor Negatives.-When inconvenient or impossible to repeat an exposure, it is often desirable to improve the printing qualities of a negative. Retouching is, of course, out of the question for scientific photographs, as alterations in detail might be introduced thereby, but there are a few permissible expedients.

Spotting is resorted to to cover pin-holes caused by dirt 
on a negative. Photopake or some pigment in water or gum-water is applied with a fine brush.

Intensification.-When a negative is too thin, or its contrasts too weak, silver, mercury, or other body is deposited on the image until the desired density is built up as nearly as possible. A mercury and a chromium formula, both veryserviceable for this purpose, are given on page 271. To ensure success it is necessary that all hypo should be eliminated from the plate by thorough washing, and that the plate be well washed between the bleaching and blackening, and after the latter. A dried plate must be immersed in water for half an hour before intensification.

If a negative is so thin that parts of it print too deeply before details appear elsewhere, yellow water-colour may be applied to the back of the negative on those parts, and if judiciously done the details may print there almost equally with the rest of the negative.

Reduction.-If negatives are too dense to print in reasonable time, but contrasts are not too great, an immersion in Howard Farmer's reducer will improve them. This solution acts first on the shadows, that is the faintly darkened parts in the negative, leaving the high lights less acted upon. The result is that contrasts are increased, but there is a danger of removal of faint details. Great care must therefore be used in its application. Only a dilute solution should be used, and a dish of water kept at hand to plunge the negative into at any moment to stop reduction. A careful application also removes fog and brightens a negative. Ammonium persulphate is another reducer with a different action; it attacks the high lights first, and consequently reduces contrast. It is occasionally useful for over-developed negatives.

Printing Processes.-The selection of a suitable printing process has no small influence on ?he production of 
a good photomicrograph. It is generally essential that the detail present in the negative be transferred to the paper without loss, but sometimes it is necessary to obtain as great contrast as possible in the print, even though this may involve a slight loss in the delicate halftones and detail. Convenience may decide whether it is better to employ a paper that is printed out in daylight, or whether a development paper that only requires a short exposure to artificial light followed by development is to be used. Most papers can be obtained in a variety of grades with glossy, semi-glossy, matt, or rough surfaces. The glossy give the most detail, and the rough are practically useless for photomicrographs.

Printing-Out Papers.-There is no paper like glossy "P.O.P." for the reproduction of fine detail, and it is generally the best paper to select for use with good negatives, when daylight printing can be resorted to. The manipulation is simple, and details are given in the packets.

Self-toning papers are the simplest of all to use, and require fixation only after printing, but as a rule some loss of detail is apparent in comparison with a toned P.O.P. print.

Development Papers are broadly divided into two classes-" Gaslight" and "Bromide." Their manipulation is practically identical, except for the length of exposure required. Bromide papers must be worked in yellow or red light; gaslight papers will not fog even if the room is lighted by gas, provided they are not exposed directly to it, and reasonable care is taken to shield them during development, etc. The paper is placed with the negative in a printing-frame, and exposed to the illuminant for the required time. Different brands vary in speed, but bromides may be expected to require only a few seconds at two feet from a 16 c.p. electric light, while gaslight papers require $\frac{1}{4}$ to several minutes at one 
foot from the light, according to the density of the negative, and if very dense may be exposed to diffused daylight for a few seconds. Correct exposure is easily found by exposing a piece of the paper beneath the negative for a time that is judged to be about $\frac{1}{4}$ or $\frac{1}{8}$ of the correct time, and then successively covering up $\frac{1}{5}, \frac{2}{5}, \frac{3}{5}, \frac{4}{5}$ of the negative with a card, and continuing the exposure at each step by times which are to the first exposure as $1,2,4$, and 8 . On development the paper will be divided into strips which have received exposures which are to each other as $1: 2: 4: 8: 16$, and the correct time can be readily judged for future exposures. The developer is then rapidly poured over the paper in a dish, and development is complete with gaslight papers in a few seconds, while bromides take 2 to 3 minutes. They are fixed in $15 \%$ hypo containing $5 \%$ potassium meta-bisulphite, and then washed well.

These three printing processes provide all that is necessary to get the best results from a wide variety of negatives.

P.O.P. will give the finest detail from good negatives.

Gaslight paper is most suitable for flat or thin negatives, or in any cases where the greatest possible contrast is desirable.

Bromide paper does not give such contrasty prints as gaslight, and should be chosen for very hard or dense negatives, and is used for enlargements.

The matt grades of the development papers are more useful than matt P.O.P., because when these papers are chosen the attainment of detail is not as a rule so important. The semi-glossy varieties can be particularly recommended.

Platinotype and Carbon Processes.-The platinotype process is not so much used for photomicrography as its merits might warrant. It suffers in comparison with the others on the score of expense and susceptibility to 
atmospheric moisture, but with care no other black-andwhite prints can compare with platinotype for beauty and permanence. Good negatives are essential, and those grades of paper with the smoothest, almost semi-glossy surface are the best: Black Japine Platinotype, for example. The matt varieties do not give such fine detail as P.O.P. The paper is printed-out in daylight until all detail is visible, then developed in potassium oxalate solution, and fixed in dilute hydrochloric acid.

Carbon printing does not render fine detail very well, and red and brown pigments are worse in this respect than the blacks and blues. Carbon may, however, be very useful in circumstances demanding prints of a definitely selected colour.

Glazing Prints.-Glossy papers are glazed in order to give them the highly polished surface which brings out fine detail to the best advantage. The finished prints are hardened in formalin, given a short wash in water, and squeegeed down while wet on ferrotype sheets or plate glass. The ferrotype sheets are previously rubbed over with waxing solution, and the glass polished with French chalk.

Enlarging.-There are several ways in which enlarging on bromide paper forms a most useful photomicro. graphic process. In certain cases, for instance, it may be impossible to use a high-power objective on account of its small depth of focus, or because its definition breaks down when used with a high eyepiece or long extension. A low-power photomicrograph on enlargement may, under these circumstances, give a much better result than a negative taken directly at a higher power. But it must always be remembered that, if anything, there will be a loss of definition and detail in the enlargement, and that the low-power objective cannot give the same resolution as the high-power. Large prints for demonstration are also much more cheaply made by enlarge- 
ment than from a large negative. An example of a highpower photomicrograph made by enlargement is given on Plate 43 , where a portion of a photomicrograph of Amphipleura pellucida, the original magnification of which is $\times 1250$, has been further enlarged, giving a magnification of 3750 times. This should be compared with the photomicrograph of Pleurosigma angulatum (Plate 42), in which the original magnification is 5000 diameters.

Many of the photomicrographic apparatus already described can be adapted for enlarging, and Fig. 69 shows

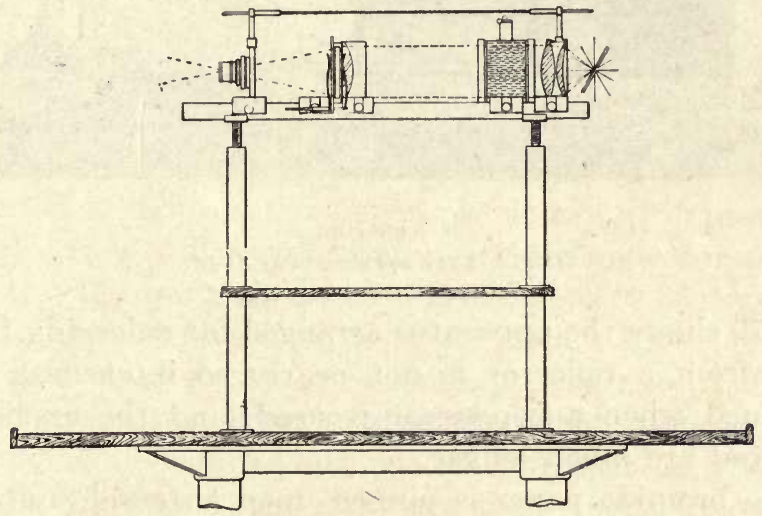

Fig. 69

OPTICAL BENCH ARRANGED FOR ENLARGING OR PROJECTION

Beck's large optical bench arranged for that purpose. The diagram of the light rays indicates that the negative is placed close to a condenser whose diameter is sufficient to cover the whole of the part to be enlarged. $4^{\prime \prime}$ or $4 \frac{1}{2}$ " condensers are usually large enough for quarterplate negatives, when the whole surface is not required. The second condenser throwing a parallel beam into the converging lens is not essential, though advantageous; a single one can be. used, focussing a convergent beam over the objective. The lamp must be carefully centred until a uniform disc of light is projected on a screen. 
- Equally good results can be obtained without a condenser at all, by throwing light through the negative from a reflector in the manner described in Chapter VII.

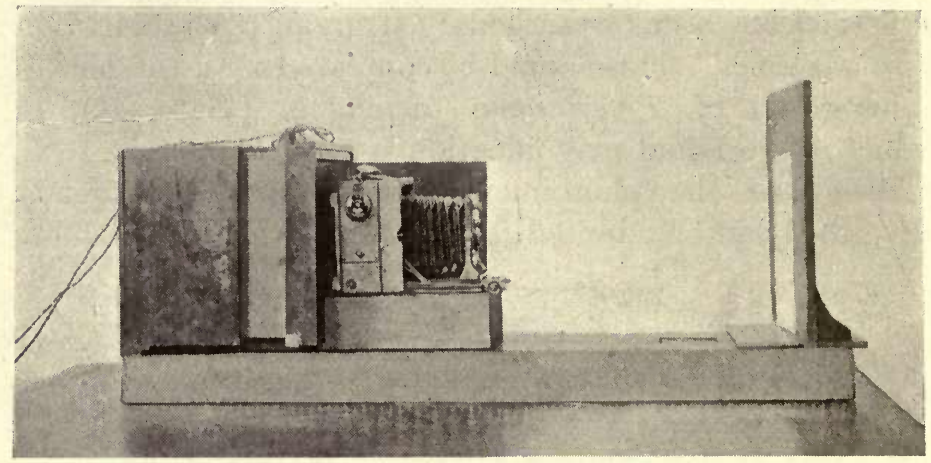

Fig. 70

SIMPLE ENLARGING APPARATUS

Fig. 70 shows the apparatus arranged for enlarging. The light from a reflector is not nearly so intense as that obtained when a condenser is used, and the exposures required are much longer.

The bromide paper is pinned upon an easel, and it is evident that no actinic light beyond that forming the image must be allowed to reach it. If enlargement is done in a dark room, a curtain or focussing-cloth can be used to cut off extraneous light from the lamp; a rod on which a curtain can be carried is shown in Fig. 69 . A better plan is to make use of a bellows between condenser or reflector and lens, as in Fig. 70. The radiant in any case is enclosed. In order to carry out enlargement in a lighted room a bellows must be used to protect the bromide paper. In Zeiss' large camera there is a central panel to carry the lens, the bellows on one side of it carries a suitable holder for the negative, while the bromide paper is placed in a dark slide fitting the other 
bellows. Focussing is carried out on the ground glass. Alternatively a large and a small camera can be used together for this purpose. Daylight can be used if desired for enlarging by pointing the camera towards a white reflector or tilting it towards the sky.

The process in brief is as follows. The apparatus is set up, and the disc of light projected on the screen carefully examined, and any unevenness corrected by moving the radiant. The negative is inserted in its carrier, and the distances between the lens and negative on one hand, and lens and screen on the other, adjusted to give the desired magnification, and the image carefully focussed. The bromide paper is placed in the dark slide or pinned to the easel, after covering the lens with a shutter or piece of red glass, and exposure given. Trials can be made on a strip of the paper previously, if exposure is uncertain; it will vary from a few seconds to several minutes, according to the illuminant, density of negative, speed of paper, and scale of enlargement. Development is carried on as usual for bromide papers, except that it is well with large sheets to soak the paper in water for a few moments before pouring the developer over it. A smooth, vigorous paper gives the best results for photomicrography: Moderately short-focus anastigmats, up to say $7^{\prime \prime}$, are the best lenses to use: the $3^{\prime \prime}$ microanastigmats are suitable if only part of the negative is required to be enlarged, but will not cover the whole of a quarter-plate. The distances required between the negative and lens, and between the lens and bromide paper, in order to get any desired enlargement are given on page 276 for lenses of moderate focal length.

Lantern Slides.-The use of photomicrographs for teaching and lecturing is becoming so widespread, that a brief outline of the process of lantern-slide making is not out of place here. Lantern plates are coated with emulsions similar to those used on bromide and gaslight 
papers, and prints are obtained on them by contact, reduction, or enlargement, in exactly the same way. Equally good lantern slides can be made on the fast and slow plates, provided the exposures are accurately gauged and development is carried to the right point, which is easily learnt by examining a few trial slides in the hand and in the lantern.

The Paget Prize Plate Co. have introduced a lantern plate called the "Hydra Lantern Plate," which can be printed-out in daylight and treated in the same way as a self-toning paper. It gives very bright slides and is less troublesome than gaslight printing and development, particularly when only odd slides are required.

Lantern Slides by Reduction.-Lantern slides are often required from negatives or parts of negatives of greater area than the lantern plate, and these must necessarily be made by reduction. Any of the enlarging apparatus described can be used for this purpose by reversing the relative distances between the lens and the negative on one hand, and the focussing screen on the other, and adjusting the two until the required reduction is obtained. The dark slide is fitted with an adapter to carry the lantern plates. Fast lantern plates are the most convenient, and require very short exposures with a good light and condenser. Fifteen seconds to a minute or two, according to the density of the negative, will suffice with the reflecting arrangement of Fig. 70 and two 32 c.p. lamps. If daylight or a good illuminant and condenser is used the exposures just mentioned are sufficient for the slow plates. After-Treatment of Lantern Slides.-Lantern slides must be bright, clean, and delicate, and with practice good ones can be made with certainty; but sometimes it is desirable to correct defects. Thin and flat slides can be improved by the methods of intensification given on page 227 , while dense or fogged slides may be brightened by a short immersion in Howard Farmer's reducer. 
There are many toning processes involving the use of copper, vanadium, gold, and other solutions, and these are very useful when brown, green, blue, or other coloured images are required. Formulæ for these and for special developing methods can be found in the almanac of the British Journal of Photography, or cartons of the salts, such as the Leto toners, may be bought for the purpose.

In many cases colouring gives a very good effect, and the process is very simple when the transparent Japanese colours or aniline dyes are used. The colour is applied in thin washes, and gives a most realistic result without calling for any great degree of artistic ability.

When finished, perfectly dry, and preferably varnished, the slides are suitably masked and bound up in contact with a piece of plain glass. Binding strips with a very strong adhesive must be used, and we have found the "Specialist" spot-binders very good : a strip with the spots on is placed along the top edge of the slide with the spots to the front, and serves as a guide to the lanternist when placing the slides in the lantern.

The Dark-Room Lamp.-The illumination of the darkroom calls for some attention on account of the almost universal use of colour-sensitive plates. The colour of the light used must be selected according to the sensitiveness of the emulsion to the various parts of the spectrum, and it should be made to give the greatest amount of light that can be used without fogging the sensitive materials. In the best lamps the light is not projected direct from the radiant through the light filter or safelight, but reflected from the back, while the radiant is screened from the front. By this means a brighter illumination can be used with safety, and there is no risk of damaging the safelight. The simple enlarging apparatus shown at Fig. 70 forms a most excellent lamp of this type if the necessary safelight is placed in the front, the camera being removed. 
A series of glass or fabric screens should be obtained, sliding in a frame over the window, if daylight is to be used, or fitting into the lamp. A canary-yellow can be used for gaslight papers, but ordinary plates must be developed in ruby light. Orthochromatic plates may require two thicknesses of ruby glass, or glass and fabric, and panchromatic plates can only be developed in darkness, or at most in a dull green light adjusted to their region of least sensitiveness. A properly made safelight by such firms as Wratten and Wainwright or Sanger-Shepherd can be relied on not to fog the plates for which it is adjusted, while an ordinary piece of red glass or fabric which does not pass nearly so much light might be quite unsafe. In any case it is advisable to expose coloursensitive plates as little as possible to light of any kind ; the dishes should be kept covered during development, and the plates examined as little as possible.

The difficulty of developing in the dim light used for orange and red sensitive plates may be partially overcome by grading the safelight. Wratten's No. 3 safelight for panchromatic plates, for instance, is made up of two glasses, one coated with a green and the other with a yellow film, bound up face to face with a piece of green paper between. The three transmit a very feeble green light, which is practically of no use unless one remains in the dark room a considerable time for the eyes to become accustomed to it, but a brighter light can be obtained by taking the binding off three edges and cutting a vertical strip about an inch bro off the green paper. The paper is then replaced and the whole bound up again, forming a screen with a narrow strip down one side which transmits considerably more light than the rest. A panchromatic plate would be fogged if exposed more than momentarily to this light, but by keeping that part shaded, and only using it for rapid examinations of the plate from time to time, or for reading the watch, de- 
velopment is made easier. A similar device may be used for red lights, and is particularly useful when two or more shutters cover a window ; one may be drawn aside and the light from the narrow section of the others used as above.

Testing Safelights.-The safety of the lamp screen can be examined by developing a plate, half of which has been exposed for ten minutes to the usual developing light, while the other half was kept covered. The development should be carried out in a covered dish for the normal time required by the developer used, and any darkening of the exposed half as compared with the other will indicate that the light must be attended to. 


\section{CHAPTER XV}

\section{SOME APPLICATIONS OF PHOTOMICROGRAPHY}

An outline of the photographic processes applied to metallography, so largely used as a control and record in the great steel and other metal industries as to constitute the most specialised and important application of photomicrography, has been already given. Apart from these, the ordinary methods detailed for low, medium, and high powers, suffice for practically any application, and there is usually little difficulty in modifying them for work requiring special treatment. In this chapter, however, some of the threads are gathered together, and those devices more particularly applicable to certain branches of science and study collected from the sections in which they are fully described. It is impossible to mention more than a tithe of the purposes for which photomicrography is employed in the control of textile, food-stuff, and other industries, or as a record of adulteration and falsification. Wherever the microscope finds application, and permanent records of observations are required, photomicrography is the readiest, most convenient and accurate means available. Prints can be stored with the written notes of analyses and examinations, and have not only the value of records of known facts, but of points unknown or unrecognised at the time, which would inevitably be missed in any written notes, but may become of the highest importance in future researches, and afford accumulated evidence which it would be impossible to obtain otherwise. Its value in 
teaching and lecturing need hardly be emphasised, and it is in this connection that the process of lantern-slide making is described.

The topics selected for discussion are confined to those illustrated by the plates; though restricted in this way they are sufficient to give some idea of the wide range of photomicrography, its value in various professions and sciences, and great superiority, in most particulars, over any method of drawing. The modifications of general processes, and the special methods described refer particularly to the plates, but may be applied with very little variation to many other subjects. In order to make the descriptions of the plates as complete as possible an outline is given of the methods employed to prepare some of the objects. They are in the main the ordinary processes familiar to microscopists, but may be found useful by some readers, as success in photomicrography depends in no small degree on suitable preparation of the specimens. Further details and devices applicable to special cases must be sought for in one or other of the many excellent text books dealing with micro-technique.

The use of Photomicrography in Pathology and Medical Practice (Plates 31 and 32).-There are many directions in which photomicrography is of service in medical study and practice, and, as the subjects which present themselves range from the most minute bacteria to entire growths and organs of considerable size, an acquaintance with the methods of high-, medium-, and low-power photomicrography is desirable. To be prepared for any contingencies a set of objectives such as that mentioned at the foot of page 81 (No. 1) should be obtained. No. 3 on page 82 is the set usually in the hands of medical men, but the omission of a lower power than $\frac{2}{3}$ " is rather a drawback.

A series of photomicrographs attachable to the notes forms an excellent record of progress in certain inoperable 
cases in which it is possible to remove small portions of a growth while under treatment ; for example, inoperable cancer under Radium treatment, and rodent ulcer under $\mathrm{X}$-rays, Ionic medication, or Carbon dioxide snow. Plates 31 and 32 are from such a series and show the change brought about in cancer by Radium treatment. Such photographs are more convenient for reference than the original slides, and quite satisfactory if typical portions of the section are picked out.

Photographic records may also be kept of conditions of which other evidence cannot be conveniently preserved; casts occurring in urine, and the changes in the blood corpuscles which occur in cases of the progressive leukæmia, may be cited as examples.

Photomicrographs of sections should be taken at both low and medium magnifications to fully demonstrate the general relationships of the tissues, and the more minute structure of their component cells. Powers of $1 \frac{1}{2}$ " and $\frac{2}{3}$ $(36 \mathrm{~mm}$. and $16 \mathrm{~mm}$.) giving magnifications of 50 to 100 diameters, and $\frac{1}{4}{ }^{\prime \prime}$ or $\frac{1}{6}$ " $(6 \mathrm{~mm}$. and $4 \mathrm{~mm}$.) objectives giving 300 to 700 times, are generally suitable. The $\frac{1^{\prime \prime}}{6}$ is advisedly of moderate N.A., about 0.7 rather than 0.9 , as the increased depth of focus is frequently of value. A green screen is the most generally useful for contrast, but an orange filter may prove more satisfactory with blue sections. It is well to employ a cooling trough to preserve the sections from the concentrated heat of an intense illuminant.

The photography of large specimens is described later under the heading "Solid Objects," and the bacteriological methods, which are rather specialised, are dealt with in the next section.

Stained Preparations of Bacteria (Plates 33 and 34).Diffraction effects, usually noticeable as white lines round the organisms, lack of contrast, and hazy images, are the faults most generally encountered in photo- 

PLATE 33.

\section{Bacteria.}
A Bacillus anthracis with SPOREs $\times 1200$
B Bacillus typhosus, FLAGELLE $\times 1200$
C Klebs-Löffler Bacillus $\times 1200$, swab from throat in diphtheria
D Spirochata plicatilis $\times 600$
E Micrococcus IN PUS $\times 1500$

A and B

C

\begin{tabular}{|c|c|c|}
\hline Objective ... & $\begin{array}{c}\text {.. Baker's } \frac{1}{12} " \text { oil immers. } \\
\text { Achromat. }\end{array}$ & $\begin{array}{c}\text { Swift's } \frac{1}{12} \text { " oil imm } \\
\text { Achromat. }\end{array}$ \\
\hline Ocular & ... Watson's Holos. $\times 10$ & Swift's No. 4. \\
\hline Condenser & \multicolumn{2}{|c|}{ Watson's Universal Achromatic. } \\
\hline Illuminant & \multicolumn{2}{|c|}{4 amp. arc. } \\
\hline Aux. Condenser & \multicolumn{2}{|c|}{ Double convex lens. } \\
\hline Screen & Yellow-green B+E. & $\mathrm{E}$ (orange). \\
\hline Plate & Wratten's “"M.”, & $\begin{array}{l}\text { Imperial Special } \\
\text { Rapid Ortho. }\end{array}$ \\
\hline Exposure & $30^{\prime \prime}$. & $2 \mathrm{~min}$. \\
\hline Developer & M.Q. & Pyro-soda. \\
\hline & D & $\mathbf{E}$ \\
\hline
\end{tabular}
Objective $\ldots \quad \ldots \quad \operatorname{Leitz}_{\frac{1}{10}}{ }^{\prime \prime}$ oil immers.
Achromat.

Zeiss $2 \mathrm{~mm}$. oil

Ocular $\quad \ldots \quad \ldots \quad$ Leitz No. 0 . immers. Apo.

Condenser $\quad \ldots$ Abbe N.A. 1.4, oiled. Zeiss projection $\times 4$.

Watson's Holos. immers. N.A. 1.30 .

Illuminant ...

Aux. Condenser

Screen

Liliput 4 amp. arc.

Double convex lens, convergent light.

Wratten's B+E, monochromatic yellow-green 5600-6000

\begin{tabular}{lllccc} 
Plate & $\ldots$ & $\ldots$ & $\begin{array}{c}\text { Wellington } \\
\text { Anti-Screen. }\end{array}$ & Imperial N.F. \\
Exposure & $\ldots$ & $\ldots$ & $30^{\prime \prime}$. & $1 \mathrm{~min}$. \\
Developer & $\ldots$ & $\ldots$ & \multicolumn{3}{c}{ Pyro-soda. }
\end{tabular}

Further photographic details and methods of preparation given on page 240.

Photos. H. L. H.

W. B. R. 


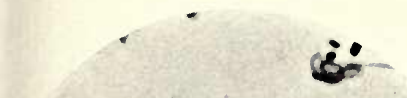

$=\frac{1}{8}=$

A
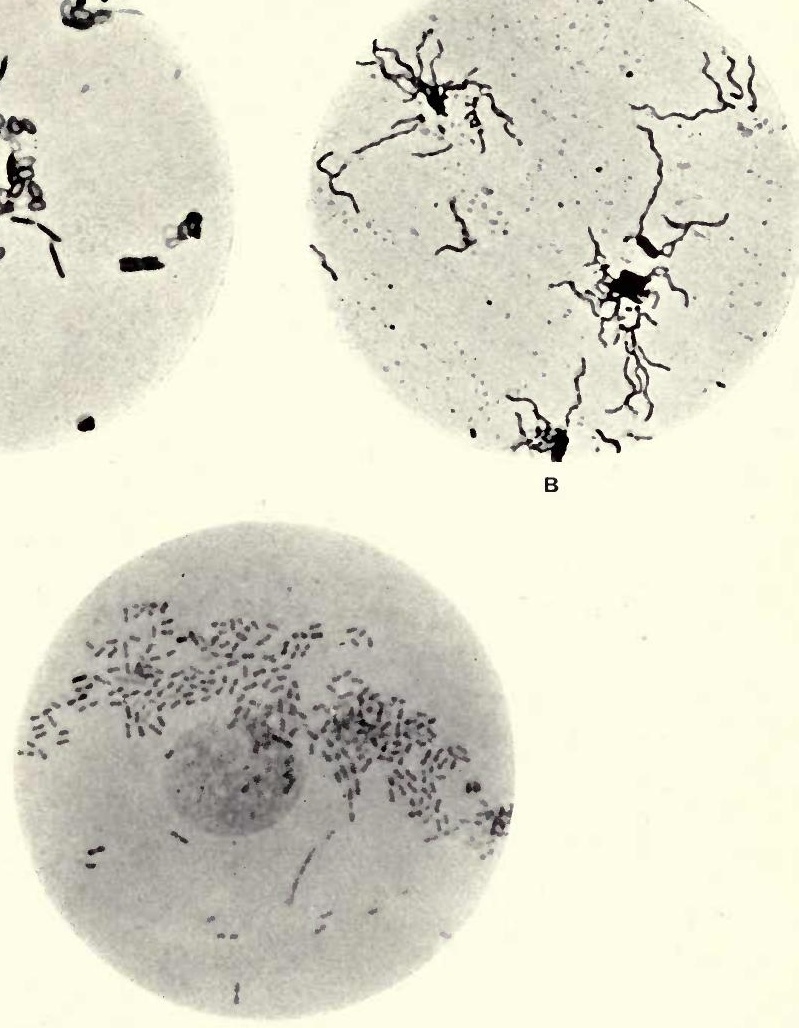

c
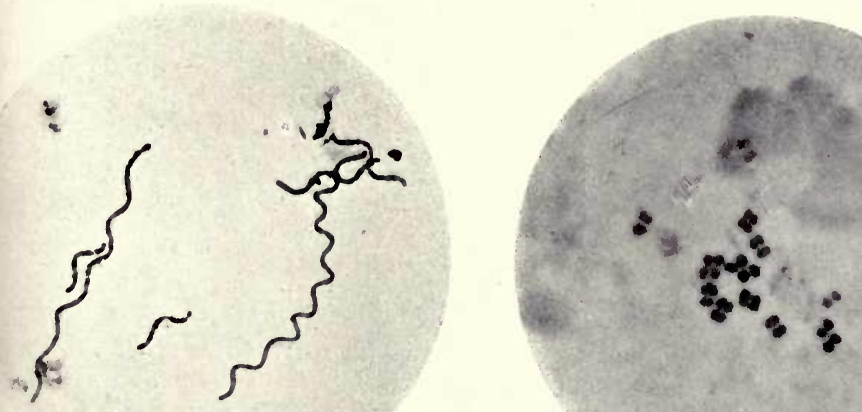




\section{Bacteria.}

A Bacteria fron an infusion of Peas $\times 1250$

The preparation contains several forms of bacteria derived from air-borne spores which have settled in an infusion of peas and developed in a few days. The most noticeable is a comparatively large organism containing granules which have taken up and hold the stain more tenaciously than the rest of the protoplasm.

Stained in gentian violet.

\section{B Bacillus tuberculosis $\times 1250$}

A cover-glass preparation of Sputum made to determine the existence of tuberculosis.

The bacilli stained red with carbol fuchsine, the nuclei of the pus cells counterstained in methylene blue.

\begin{tabular}{|c|c|}
\hline & $\frac{10^{\prime \prime}}{10}$ oil immersi \\
\hline Ocular $\quad \ldots$ & Zeiss Projection $\times 4$. \\
\hline Condenser & $\begin{array}{l}\text {.. Watson's Holos. immersion, N.A. 1.30, oi } \\
\text { slip, full aperture. }\end{array}$ \\
\hline Illuminant & .. Liliput 4 amp. arc. \\
\hline Aux. Condenser & $r$ Double convex lens, giving parallel be \\
\hline Screen & .. Wratten's B (green) and E (orange). \\
\hline Plate & Imperial N.F. \\
\hline Exposure ... & .. 5 sec. and $10 \mathrm{sec}$. \\
\hline Developer & ro-soda. \\
\hline
\end{tabular}

The manipulative details for the photomicrography of bacteria are given on page 240 . The use of a wide cone of light, and of yellowgreen monochromatic light $\lambda=5600-6100$ to increase contrast and improve definition should be noted.

The methods of preparation are given on page 242 .

Bacteria as test objects. The sharpness of the images of bacteria taken under these conditions is a good criterion of the qualities of an objective. The beaded structure of B. tuberculosis is characteristic. 


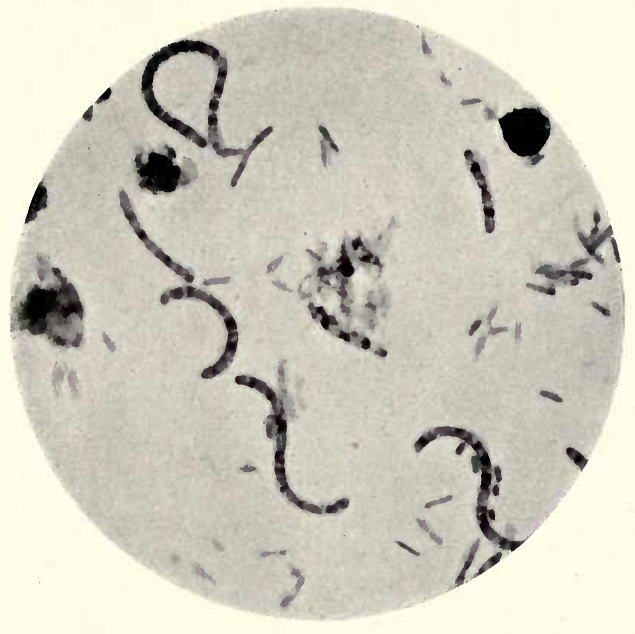

A

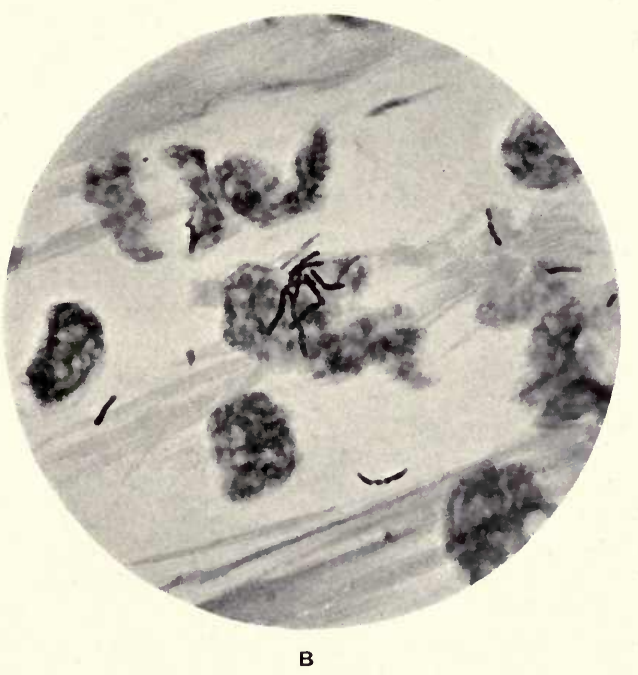






\section{Photomicrography of Blood Parasites.}

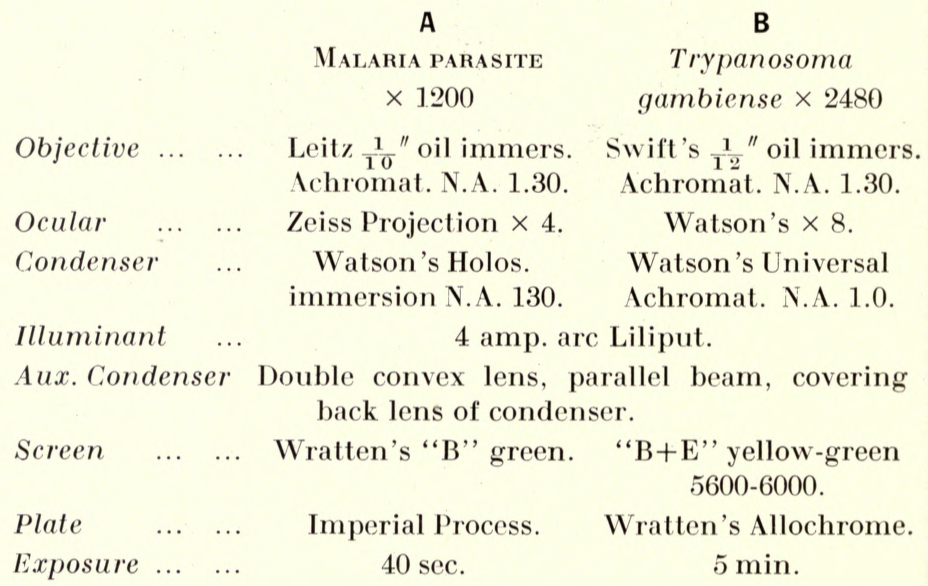

Both these organisms are parasitic Protozoa occurring in the blood. That of Malaria exhibits one of the phases of its life-history, known as the "crescent stage." The Trypanosoma gambiense is the cause of sleeping sickness.

Both preparations coloured bluish-violet with red nuclei by means of Leishman's stain. 


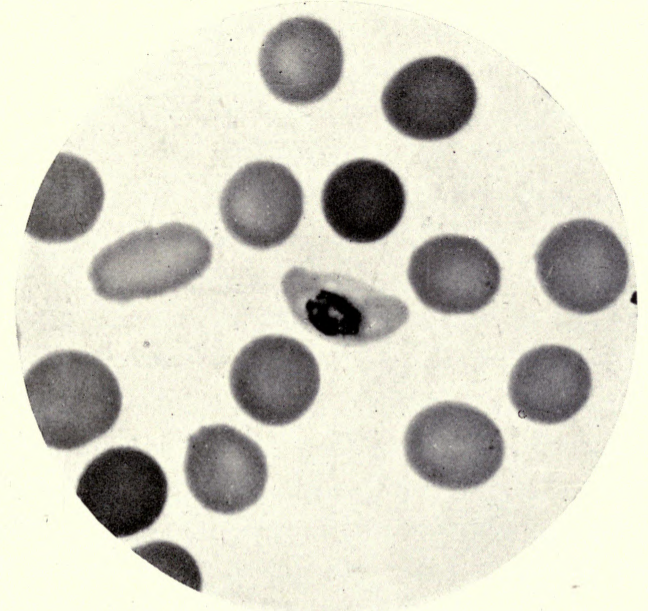

A

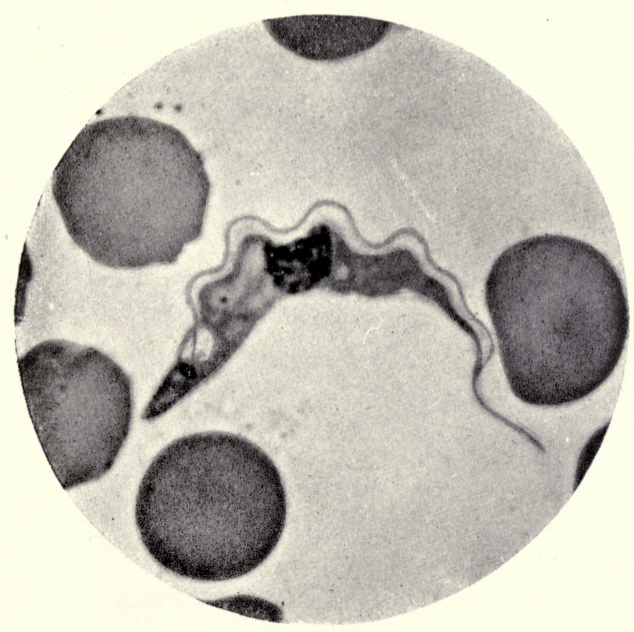



micrographs of bacteria, etc., and every effort must be made to obtain a sharp, distinct image. A good objective and proper adjustment of the illumination are essential to success. Freedom from diffraction effects is ensured by the use of the largest a vailable aperture ; oil-immersion objectives of $\frac{1}{10}$ " and $\frac{1}{12}$ " are almost always employed, and it is advisable to use an immersion condenser, although an achromatic condenser of N.A. 1.0 usually gives perfectly good results if its back lens is filled with light. A wide condenser aperture also has the effect of eliminating the structure of the more faintly-stained tissues of sections in which bacteria occur, and so enhancing the contrast of the well-stained bacteria.

To get proper contrast the preparation must be a good one and sharply stained, but now that several good series of contrast screens are available the colour of the stain used is not of such great consequence as it was before their introduction. A screen with a transmission band. complementary to that of the stain works wonders in improving contrast, particularly if its colour is suited to the corrections of the objective, but it is difficult to get even passable results from faded preparations. A table is given on page 267 indicating the most suitable screens for use with various stains. Panchromatic plates are generally to be preferred, and are essential with some screens to obtain the best results and allow of reasonably short exposures, but in the majority of cases orthochromatic plates are satisfactory. Slow or medium plates, particularly process plates, should be used. when possible in preference to the very fast varieties.

The tricolour process is much more suitable than the screen-plate processes for the colour photography of bacteria, on account of the clearness of the white background and freedom from granularity, but a two-colour process, or a single dyeing of the positive on bichromated 
gelatine in the colour with which the bacteria are stained, is generally all that is required.

Preparation of Bacteria for Photomicrography.-The photomicrographs of Bacillus typhosus and Bacillus anthracis were from preparations by Baker; the typhoid bacillus is stained by a process of deposition in order to render the flagellæ distinct, and in consequence a deposit of black spots over the field is unavoidable. Very simple methods, of which the following is an outline, were employed for the other preparations. A small drop of the growth in liquid culture medium, or a small portion of sputum, etc., is placed on a cover-glass, covered with another, and the twoglasses pressed together withfingerand thumb to obtain as thin and even a film as possible. The cover-glasses are then slid apart and set aside to dry, and afterwards passed rapidly through a Bunsen flame three times to fix the bacteria. The staining process which follows differs for the different organisms. Formulæ for the stains are given on page 266.

Spirochceta plicatilis from water containing decaying algae. Spirillum from an infusion of Peas (Plates 33 and 34).These were stained by the simplest process, immersion of the cover-glass in a dilute solution of the dye, Gentian violet, for a few minutes, followed by a wash in water, and afterwards in $\frac{1}{2} \%$ acetic acid if too deeply stained; finally washed in water and put aside until quite dry, or dehydrated in absolute alcohol and cleared in xylene. A drop of Canada balsam was placed on the centre of the preparation, the cover-glass turned over on to a slip, gently pressed, and the slide allowed to dry.

Swab from the Throat of a Diphtheria Patient.-Plate 33.C showing the Klebs-Löffler bacillus. The preparation was immersed in Löffler's methylene blue solution for 3 minutes, washed in water and finished off as above.

Bacillus Tuberculosis from Sputum (Plate 34.в).Carbol-fuchsine warmed in a test-tube was poured on 
the warmed cover-glass preparation and kept 2 minutes. The excess of stain was blotted off, and the cover-slip immersed in $25 \%$ nitric or sulphuric acid The retention of the red colour by Bacillus tuberculosis under this treatment is characteristic. The preparation was washed for 1 to $1 \frac{1}{2}$ minutes in tap water, and counterstained by immersion in a saturated watery solution of methylene blue, or in Löffler's methylene blue.

Micrococcus in Pus (Plate 33.E). - Stained by Gram's method (page 266), by which the bacteria are stained but the pus cells are decolourised. This was followed by immersion in Bismark brown until the pus cells took a light brown colour, but owing to the use of a yellowgreen screen the brown comes out light and the purplestained bacteria very dark.

Living Yeasts, Moulds, Bacteria, etc. (Plate 36).Research in connection with the life history and functions of various species of yeasts and other microorganisms forms a very important part of the scientific control in Brewing and the Fermentation industries, and a true photomicrographic record of the appearance of the organisms met with is of great value. Bacteria are almost invariably prepared for photomicrography by some fixing and staining process, but the application of heat or reagents involves a certain amount of risk of alteration in the appearance of any microorganism, and this is particularly the case with yeasts and moulds, which many workers consequently prefer to photograph in their living state. There are, however, many difficulties to be encountered arising from their small size, their transparency and slight differentiation from the surrounding field, and from their motion in the mounting fluid. They are usually mounted in a drop of water or of the culture medium in which they are growing, with a cover-glass lightly pressed down on it. There is not much risk of damage to the smaller organisms if the 
slide is turned over on a sheet of blotting-paper and a slight even pressure applied, but yeast cells are very easily ruptured by a more localised pressure such as may be given by accidentally bringing the objective down on the cover-slip. It is necessary to apply a certain amount of pressure in order to get as thin a film as possible, otherwise it may be difficult to find a field in which the organisms all lie in one plane. The mount should be laid aside for some time to allow the cells to settle down on the slip, and it is as well to ring the cover-glass with melted paraffin wax or thick oil to prevent evaporation of the liquid and consequent movement in the preparation during examination and exposure. Every care must be taken to prevent such movement among the organisms, and a vertical camera is much the most suitable for this reason. Bacteria may remain in motion, vibratory or otherwise, for a long time, and the only possible way to get a sharp picture is by means of a shutter with speeds of $\frac{1}{15}$ to $\frac{1}{100}$ of a second. A very intense light is needed for such exposures with high magnifications ; a 4-ampère arc or limelight is suitable, and the use of a cooling-trough is advisable as the preparations are very susceptible to heat.

In order to obtain sufficient contrast between the organism and background the iris of the substage condenser is closed until only about half of the aperture of the objective is utilised. Extreme care must be exercised in manipulating the diaphragm, as it is very difficult to get the necessary contrast without producing a white diffraction ring round each cell. The diffraction effect may make itself very apparent in the photograph even when very difficult to detect during the preliminary examination of the object and adjustment of the iris. A green screen may be used with advantage, but it must often be dispensed with in order to allow of a sufficiently short exposure, and the best results are obtained on panchromatic plates. 



\section{Living Micro-organisms.}

A Micrococci found in Ropy BeER $\times 1000$

B Brewing Yeast, Saccharomyces cerevisice $\times 700$

C Spirochaeta pallida, PURE CUlture $\times 1000$

D Brewing Yeast $\times 700$

E A “WiLd" YeAst, Saccharomyces $S p ? \times 700$

A tendency to collect together in masses is characteristic of this yeast.

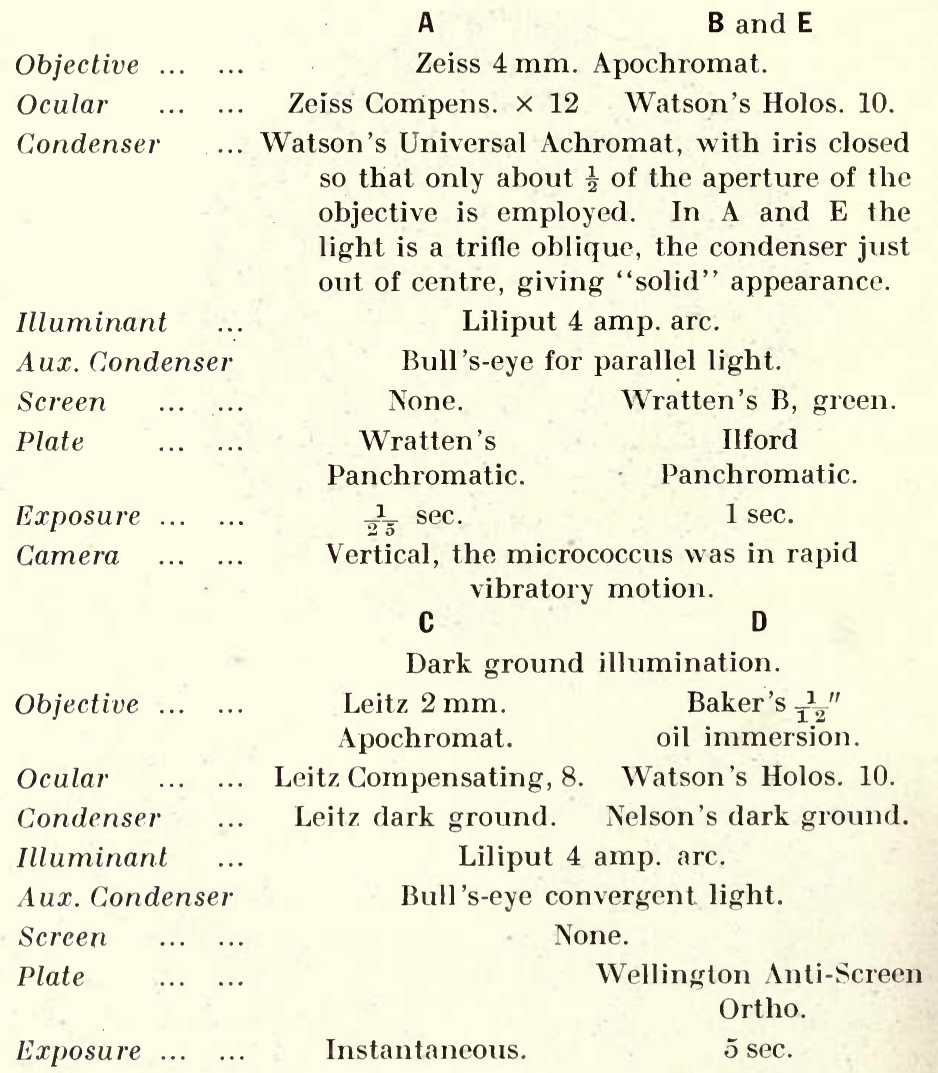

Details of manipulation given on page 243 . 

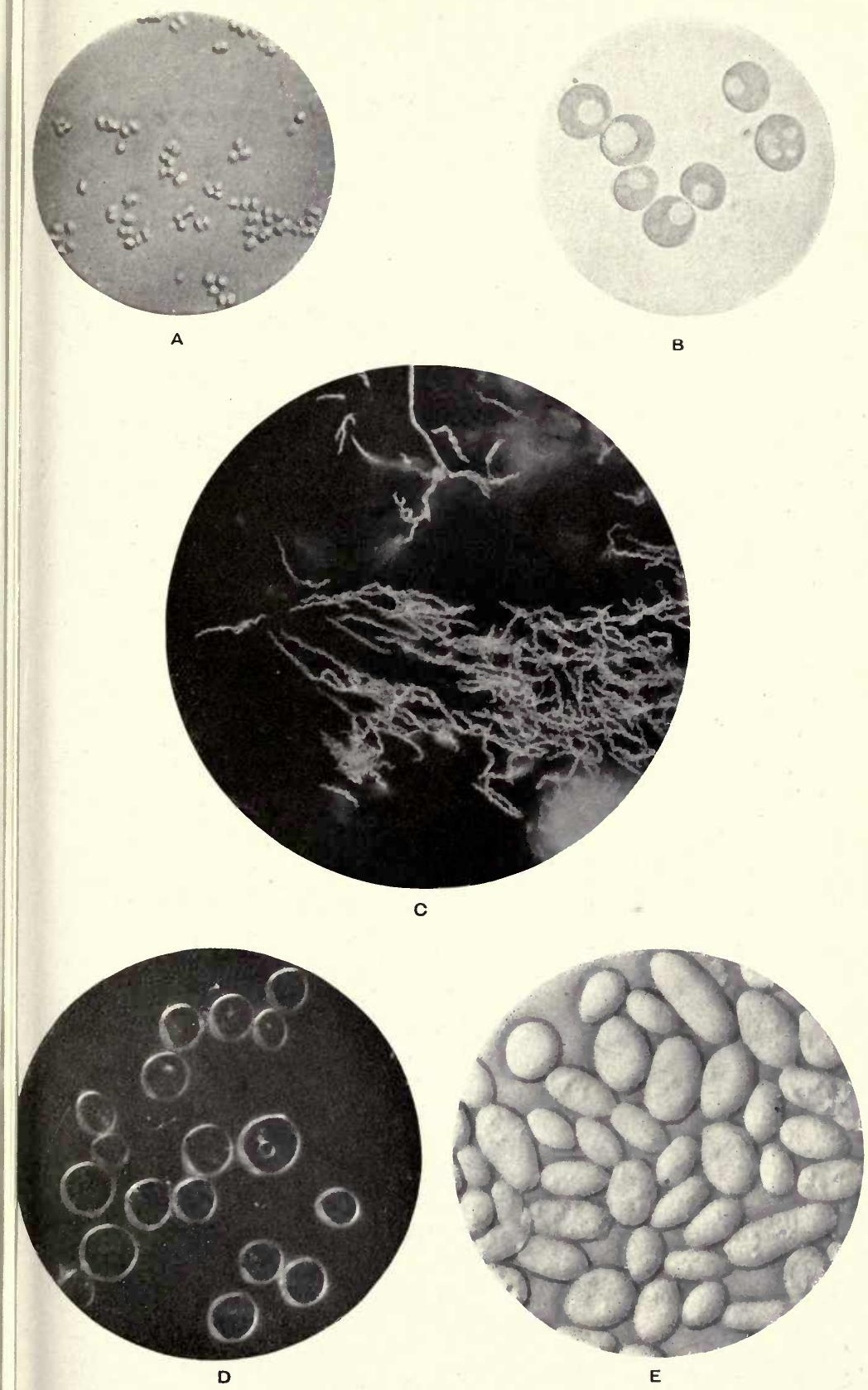

Magnifications of about 1000 are required for bacteria, but 500 times is enough for yeast cells, and even lower magnifications are ample for some moulds. The most useful lenses are the $\frac{1}{12}$ " or $\frac{1}{10}$ " oil-immersion with a medium eyepiece, a $\frac{1{ }^{\prime \prime}}{8}$ with $a \times 6$ or $\times 8$ eyepiece, or a $\frac{1}{6}$ " apochromat with a $\times 8$ or $\times 12$ eyepiece. The dry lenses have the advantage that they do not disturb the cells by communicating movement through a film of oil, but they require relatively longer exposure than the immersion objectives.

The high-power dark ground illuminators are useful for living micro-organisms, particularly for the rapid diagnosis of such forms as Spirochoeta pallida, etc., which otherwise require special staining methods for detection; a reference has already been made to their use in cinematography. The living cells in a mount of yeast are shown with a very bright outline, while dead or shrivelled cells can be rapidly picked out by their shining appearance. An arc lamp is required to allow of exposures of $\frac{1}{30} "$, but the more sluggish or non-motile organisms can be photographed with a less powerful illuminant, a Nernst for example. Two photomicrographs of living organisms on a dark field are given on Plate 36 .

It is very easy to produce alternative or even false appearances in a picture that depends so largely on diffraction for the display of its detail, and, in fact, the colourless transparent protoplasm of bacteria or yeast when photographed by transmitted light can be represented by dark structure on a light ground, or by light on a dark ground. The former is more frequently used, and Plate 36.в is an example, but the white image gives a much better idea of the solidity of the cell (Plate 36.A and $\mathrm{E}$ ). Either image can be obtained at will by a careful adjustment of the condenser and mirror, or even by a slight alteration in focus, and that which shows best the structure or detail required should be chosen. A slight 
obliquity of illumination, produced by decentring the substage condenser to a very small extent, gives the solid image which more nearly reproduces the contour of the cells than the flat image obtained by exact central illumination.

Culture Tubes and Plate Cultures (Plates 37 and 4).A difficulty arises in the photography of culture tubes from reflections on the tube, which give white lines in the print and quite obliterate the detail of the growths. This is easily overcome by immersion of the tube in a trough of water, alcohol, or glycerine, as shown in the Plate. The reflections are there noticeable on the portions of the tubes above the water. Flat-sided light filter or cooling troughs can be used for this purpose, or cleaned photographic plates may be cemented together with an aquarium cement, such as that given on page 276 . Condensed moisture on the inside of the tubes should, whenever practicable, be removed by means of a roll of filter paper.

The structure of most growths is best shown up by illumination from the front, but in some cases illumination from behind gives a better result. The simple apparatus of Fig. 40 is suitable in either case, the tubes being placed close to the background and illuminated from one or both of the lamps according to the shadows required, or brought in front of the lamp reflectors for transmitted light reflected from the white background. If a more intense illumination is required the principle remains the same, but condensers are used to throw light from one or more lamps in front of or behind the tubes, at whatever angle gives the best relief and shows the -structure to the greatest advantage. The lens is shaded from direct light from the lamp. Diffused light, daylight or artificial, may be more convenient than the above; an electric are without a condenser was used for Plate 37. White or black backgrounds are selected according to the nature of the object. 



\section{Photography of Culture Tubes.}

1. Cunture of Bacillus prodigiosus.

2. Culture of Sarcina.

Direct colour photograph. Screen-plate process (Autochrome).

Objective ... ... Goerz “Dagor”' Anastigmat, 7", by Ross. Illuminant $\quad$... Arc, diffused light.

Plate $\quad \ldots . . .$. Autochrome.

Screen $\quad \ldots \quad \ldots$ Lumière's special screen for arc-light.

Exposure ... ... 15 secs.

The production of the pigments is typical of the growth of these organisms on various solid media. The $B$. prodigiosus causes the red spots on bread giving rise to the appearance known as "bleeding bread."

This photograph is reproduced with the object of showing the device employed to prevent reflections on culture tubes, \&c. The tubes are immersed in water in a flat-sided trough, whereby the reflections, visible on the top half of the tubes, are obviated. 


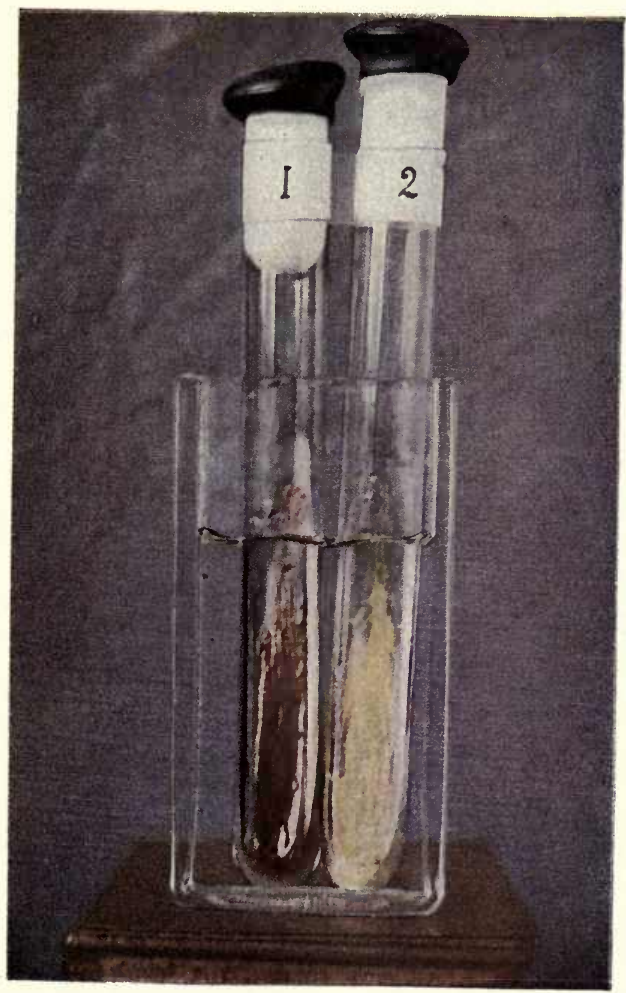



The colonies on plate cultures are photographed in a similar manner, illuminated from the front or from behind with reflected light, or by means of condenisers placed at suitable angles. Proper contrast for light-coloured colonies is obtained by supporting the plate or Petri dish some distance in front of a black background and illuminating from the front. Small colonies are sometimes displayed to the best advantage by dark ground illumination with objectives of $\frac{2}{3}$ to $2^{\prime \prime}$ focus. For this purpose the plate is laid on the microscope stage and a wheel-stop placed beneath the condenser (Plate 4.в).

Photography of Larger Micro-organisms in the Living State.-Pond Life, etc. (Plate 38).-In order to take a photomicrograph of the minute animals and plants found in ponds, etc., whilst alive and perhaps moving with considerable rapidity, instantaneous exposures are essential, and some means for focussing and watching the organisms right up to the moment of exposure must be devised, in order that they may be taken at the moment when properly expanded and in a good position in the field. A reflex camera fitted up in the way described on page 13 is well adapted to the purpose, but the Leitz demonstration eyepiece shown at Fig. 71 is in many respects even better. The special eyepiece is placed in the microscope tube like an ordinary eyepiece. It has a lateral tube along which some of the image-forming rays are directed by a reflecting prism through a second eyepiece, giving an image which can be examined direct, or projected on to a suitable surface and there focussed right up to the moment of exposure, and watched during exposure if desired. There is no chance of vibration from the movements of a mirror, and no resetting required as in the reflex camera. In the figure the card on which the image is focussed is fixed in a clamp; with a horizontal camera the card or paper screen may be placed on the table. 
To take a photograph the light and condenser are adjusted as usual, and an image of the object sharply focussed on the ground glass of the camera. The lateral eyepiece is then adjusted, by rotating the top lens, until the image it forms on the card is simultaneously in focus with that projected on the ground glass. The dark slide

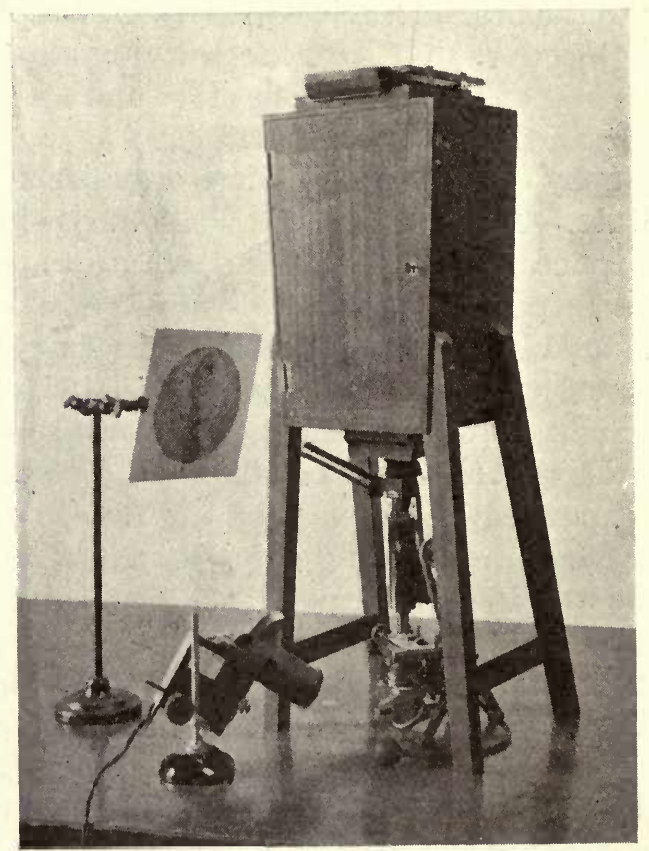

Fig. 71

APPARATUS FOR LIVING ORGANISMS

is then introduced, the automatic shutter situated above the eyepiece set to the time required, and attention directed to the image on the card until the proper instant for exposure presents itself. Alterations in the focus to sharpen the image on the card will have the same effect on the image in the camera. The loss of light due to the 



\section{Instantaneous Photomicrography.}

A Daphnia pulex $\times 20$

B Cyclops quadricornio $q \times 18$, dark ground illumination

Photographed from living specimens by means of the apparatus deseribed on page 247 .

Objective ... ... Swift 1" Achromat.

Ocular ....... Swift No. 2.

Condenser ... A. Watson's Universal Achromat, top lens removed.

B. Abbe, with wheel stop.

Illuminant ... Liliput 4 amp. arc.

Aux. Condenser Biconvex lens, parallel light.

Plate $\quad \ldots \quad \ldots$ Imperial Special Sensitive.

Exposure ... … A. $\frac{1}{25}$ sec.

B. $\frac{1}{10}$ sec.

Developer ... Pyro-soda.

These are two minute crustaceans belonging to the group Entomostraca. They abound in ponds and ditches and swim very actively. Daphinia is known as the "water flea." Cyclops is so named from its possession of one single cluster of ocelli only. The individual photographed is a female with two egg sacs attached; in these the ova undergo the earlier stages of their development.

C Portion of Cinematograph Film of living Trypanosomes of Sleeping Sigkness among Blood Corpuscles $\times 400$

Dark ground illumination.

Photos. A and B, W. B. R.

C, Messrs. PATHÉ FRÈRES. 


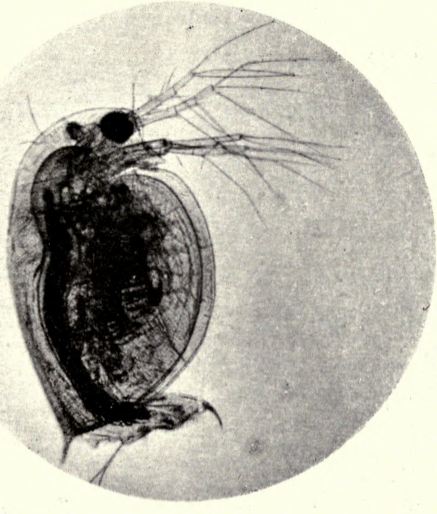

A
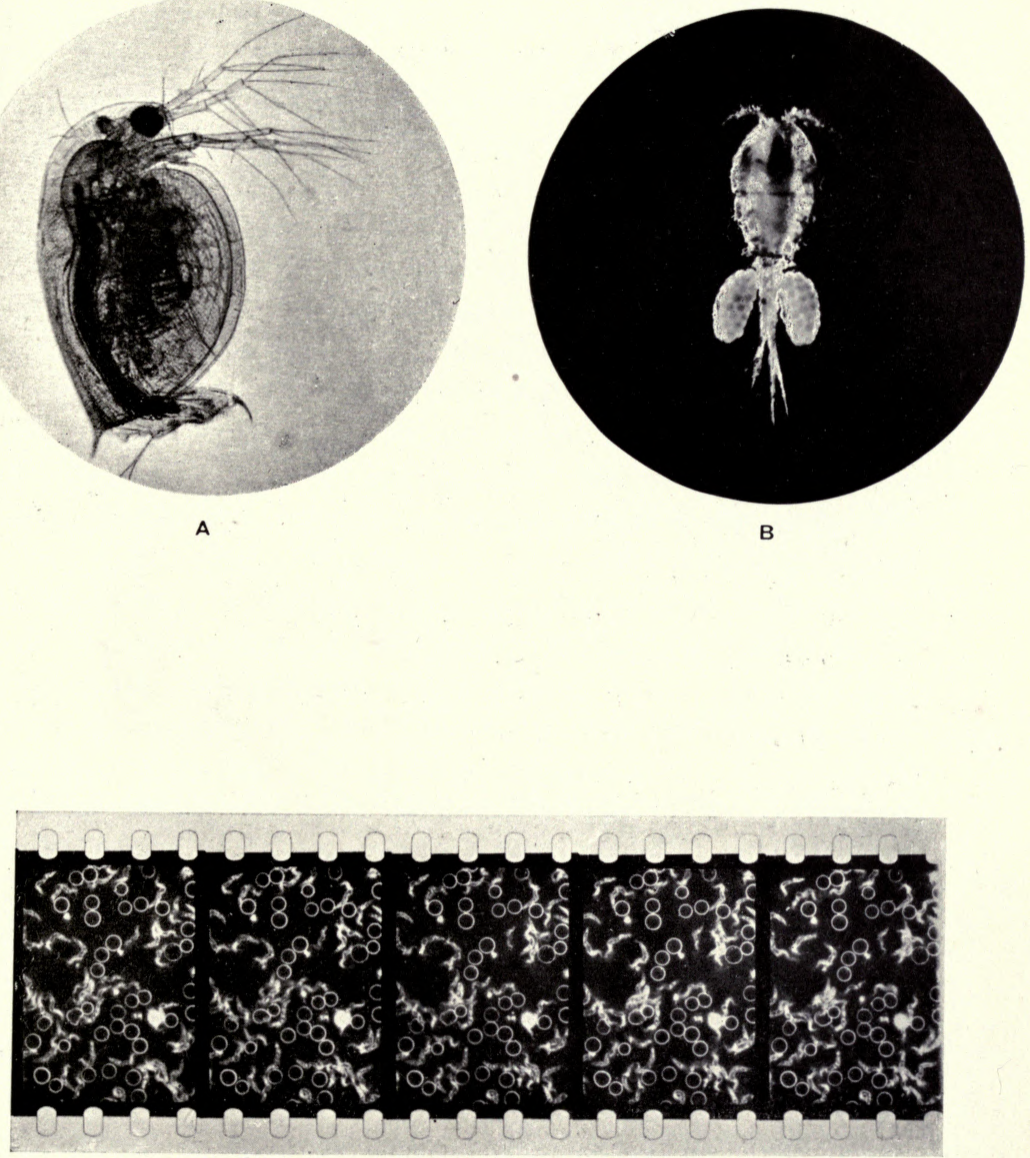

C 

prism system in the eyepiece is not great, and very short exposures can be given with a good light. Photographs taken with this apparatus are reproduced on Plate 38 .

Dark ground illumination was used for Cyclops quadricornis, and is particularly useful for such forms as Rotifers and Polyzoa. The preparation of permanent mounts of such delicate organisms as Rotifers is a difficult operation, involving a very careful narcotisation, followed by killing and fixing at the right moment with $\frac{1}{4} \%$ osmic acid or Hermann's solution. They are mounted in $2 \frac{1}{2} \%$ formalin on slides with a central depression. This difficulty in obtaining lifelike permanent mounts is a strong argument in favour of photography of the living organism whenever possible, or when the rapidity of their movements is not too great. Advantage must often be taken of some method to slacken the movements sufficiently; in some cases, Cyclops for instance, gently warming the slide will effect this. Mr. C. F. Rousselet, F.R.M.S., whose work on Rotifers is so well known, advises cocaine as a narcotic, and the solution given on page 268 may be added drop by drop to a watch-glass of water in which the Rotifers are swimming until their movements are seen to be slow enough to permit of a suitable exposure.

Photomicrography of Solid Objects.-The following notes apply to a very wide range of subjects; natural history specimens of all sorts, small objects of virtu, and the like, that can be photographed entire. Some specimens may be dry, others preserved in fluid ; some require a microscope for adequate magnification, for others a microscope cannot be employed, and a camera with a short-focus anastigmat lens is used. They are illuminated by sunlight or diffused daylight, or by lamps in conjunction with condensers or reflectors. The arrangement of the illuminant is important, as good contrasts and an appearance of relief and solidity depend on its proper management. Either vertical or horizontal cameras can 
be used, but the vertical arrangement has several points in its favour ; the object can be readily laid on a table below it, and the camera can so easily be carried into the best position for daylight. Colour photography is frequently of great value, and stereoscopic photomicrography finds its greatest application in this field. Plates 3 and 27 are examples.

Objects in Preservative Fluid, Dissections, etc. (Plates 2.A and 26.B).- Specimens preserved in bottles should be removed when possible and immersed in a dish of the preservative fluid or water for photography. Immersion in a liquid has the effect of considerably increasing the apparent depth of focus of the objective, and greatly improves the appearance of many specimens by supporting hairs, tentacles, and the like. Tremors on the surface of the liquid must be carefully guarded against. A vertical camera as illustrated at Fig. 42 is the most convenient to use under these conditions.

The Chick in Egg (Plate 2.A), and the Spider (Plate 26.в), were immersed in water and spirit respectively, a piece of black paper, previously soaked in spirit to prevent the formation of air bubbles on its surface, being laid on the bottom of the dish to form a suitable background. Plasticine can be used to support the object firmly. Specimens that cannot be laid in a dish or supported therein may be suspended in a flat-sided trough, the most convenient arrangement when a vertical camera is not available.

The precautions against reflections, detailed in the section on culture tubes, must be observed when photographing delicate specimens that cannot be removed from the jar in which they are preserved.

Dry Specimens (Plates 26.A and 4.A).-The advantage of immersion in liquid was referred to in the last paragraph, but immersion is not always applicable or desirable, and in such cases some simple device must be 
adopted to support the specimen and display it to the best advantage. The apparatus of Fig. 41 is specially made by Leitz for the photography of small insects. The specimen is carried on a needle attached to a special stage and is illuminated by light reflected from two lamps, one of the best posible arrangements for the class of objects under discussion. The simple home-made device of Fig. 40 is equally efficient, and the specimen can be supported on a special holder or by plasticine. Shadows cast on the background, and an appearance of granularity due to its rough surface, are prevented by supporting the specimen some distance in front of it. When a vertical camera is employed the object is placed on a piece of clear glass about 2 or 3 inches above the velvet or paper against which it is displayed. Small specimens such as the Leg of the Honey Bee and the Group of Shells (Plates 26.A and 4.A) are mounted on a slide and laid on the microscope stage, with the piece of black paper or velvet on the substage ring and quite out of focus.

Parts of an object illuminated by lamp and condenser are liable to be in deeper shadow than is desirable, and a great improvement is produced in the photomicrograph by placing one or more reflectors of white card in suitable positions above or at the sides to relieve this.

Insects-Transparent Preparations (Plates 13, 14 and 20).-Entire insects are largely photographed by the methods mentioned in the last section, but they are also very often mounted as transparent preparations, and considerable difficulty is sometimes encountered in their photography owing to the very great contrast between the body and wings. Either the wings are obliterated by over-exposure before any detail is given in the body, or the body appears as a black mass when the wings are correctly exposed. The same difficulty occurs with other objects, but perhaps most frequently with insects, and was referred to on page 163, where the use of a screen 
with the same transmission as the dark parts of the preparation was indicated. The body of an insect absorbs most of the blue light, and in consequence, under ordinary conditions, is shown in very great contrast to the wings or other parts which transmit blue. If blue light is excluded by a screen, and the photograph taken by red light, the difference of exposure required by the clear and brown parts is much reduced, and both will be properly reproduced. Panchromatic plates are evidently the best to use under the circumstances, and Plate 20 shows the difference obtained by photographing in blue and in red light. As a rule, however, orthochromatic plates are quite satisfactory for a well-cleared insect, but detail in the yellow parts will almost invariably be lost, and brown or yellow markings emphasised if a non-colour sensitive plate is used; both these defects are shown by the photograph of the Window Gnat (Plate 14.A). In extreme cases it may be well to increase the contrast of very delicate preparations by means of a green or blue screen, and this was done for the Flea (Plate 13) and the Louse (Plate 14.B). The Louse was so transparent and the balsam had become so yellow, that it was necessary to increase the contrast as much as possible.

The delicate hairs which surround many insects are easily obliterated by halation, or appear double or surrounded by white lines by diffraction (Plate 12), and care must be taken to avoid these defects in the photomicrographs by using backed plates, and not closing the substage iris too much.

Preparation of Insects. - A good photomicrograph of an insect depends so much on the preparation that the method employed for clearing the specimen and making it as transparent as possible is given.

Immerse in $10 \%$ solution of caustic potash until soft; this requires times varying from a few hours to weeks, according to the nature of the insect. 
Wash in water, and soak in glacial acetic acid for a day or more, then wash in water again.

Place on a slide in a saucer of water, and arrange the appendages by means of a needle and brush; raise the slide from the water, drain it, and make final adjustments of the legs, wings, etc.

Lay another slide down on the first, and tie the two together. Place in methylated spirit for a day or more to harden the object.

Remove the specimen from between the slides and place in methylated spirit.

Clear in clove oil.

Soak in xylene for a few minutes to remove the clove oil, and mount in Canada balsam.

Photomicrography of Diatoms (Plates 39 to 43).Diatoms present some of the greatest difficulties encountered in photomicrography. In visual work the greatest care in making all adjustments of the microscope has to be taken in order to display their structure to the best advantage, and this holds good, but to a greater degree, in photomicrography; for though the detailed structure of many diatoms ean be made out with comparative ease visually, provided the focussing and other adjustments have been carefully earried out, yet it is often difficult to obtain a satisfactory photomicrograph of the object, owing to lack of sufficient contrast. In fact this lack of contrast is one of the chief difficulties to be overcome, particularly with high-power work. Apart from the satisfaction to be obtained from photographing such beautiful objects, the experience gained in making the necessary adjustments is very beneficial to the photographer, and there is scarcely any type of object which offers such good training in accurate focussing, in teaching the value of critical illumination, and in general photomicrographic technique, as diatoms ; and, as the structure of typical forms such as Navicula 
lyra, Surirella gemma, etc., is fairly constant, the beginner has the advantage of being able to compare his results with figures and descriptions published by other workers, and as test objects they are of the greatest value.

Critical and monochromatic illumination, and objectives of great numerical aperture and perfect corrections are needed to give the best results. Apochromatic objectives are very desirable, but good results are to be obtained with achromats and suitable screens. The most useful powers are $\frac{1 "}{6}, \frac{1}{8}$, and $\frac{1}{12}$. The colour filters should transmit a light approximately monochromatic, and of small wave length to increase resolution as much as possible. On account of the difficulty of focussing with blue light, green screens are easier to use, and achromatic objectives generally work to better advantage with green light. In certain cases, owing to insufficient contrast, it is impossible to get all detail brought out by axial light, and oblique illumination must be resorted to, but great care must be exercised in its use, and it should only be employed to show up in greater contrast structures that can already be made out by axial light. The excessive use of oblique light tends to the formation of false images, and it is no uncommon thing to be able to make out and photograph dots or striæ lying outside the diatom, which must, of course, be purely optical phenomena. When using objectives of high numerical aperture it is often necessary to cut down the aperture slightly by means of the iris diaphragm of the condenser, in order to prevent flooding of light and to get contrast. Great care must be taken in closing down the iris as diffraction effects are so easily produced (Plate 12, Fig. D).

It is advisable to commence work on one of the large, flat diatoms, such, for example, as Arachnoidiscus (Plate 39.A), and to take the photograph with a low objective and long camera extension; there will be no great resolution, but such a diatom calls more for flatness of 
field and penetration, and these could not be obtained by using an objective of high N.A.

A comparison between the use of low- and high-power objectives for diatom photography is seen in the illustration of Triceratium (Plate 40). In Fig. A, the diatom was photographed with a low-power objective and long camera extension; the whole of the diatom is sharply rendered, but there is no trace of secondary structure; whilst in Fig. B, which is of identically the same magnification, but taken with an objective of higher N.A., the secondary markings are clearly visible, but the photograph is sharp only in the centre; that is, resolution has been obtained at the expense of penetration.

Actinoptychus heliopelta (Plate 41, Figs. A, B) offers another example of the necessity of employing a lowpower objective to obtain a sharp photograph of an entire diatom. This object is by no means flat, and it is necessary to use a narrow cone of light from the substage condenser to obtain sharpness. When photographed at higher magnification and with an objective of high N.A. the secondary structure can be resolved without difficulty.

Navicula lyra (Plate 41, Fig. D) is a favourite diatom for photography. The dots can be made out with a good $\frac{1}{6}$ " objective. They should appear perfectly white, and do so if the objective is an apochromat, but with an achromat they are generally tinged with colour, but by using a green screen excellent photographs can be obtained.

Navicula crabro (Plate 41, Fig. D) presents no special difficulty; we have photographed this diatom for the purpose of showing how easily diffraction effects are produced by closing the iris diaphragm too much. Two negatives were taken with Swift's $\frac{1}{4}$ " objective of N.A. $0 \cdot 88$, in one case working with nearly full aperture, in the other case with the iris closed down to greatly restrict the working aperture of the objective. In the illustration 
concentric lines, due to diffraction, surrounding the diatom can be easily made out on the right half of the figure, and there are also round marks on the background due to minute specks of dust which have been brought into prominence by the narrower cone of light passing through the eyepiece.

Pleurosigma angulatum (Plate 41, Fig. C, and Plate 42 ) is one of the most difficult of diatoms to photograph. The secondary markings can be made out either as white dots, circular apertures or hexagons, or, by a slight alteration of focus, as black dots with a white surround. There is a diversity of opinion as to which is the correct focus; the white dot is undoubtedly the easier to photograph. We give two illustrations of the diatom, both taken with axial illumination; one at medium magnification (Plate 41, Fig. C) was taken with a student's microscope and home-made box camera, merely to show that, if care is taken, satisfactory photographs can be obtained with the simplest form of apparatus possessing none of the refinements and accessories that are often regarded as absolutely necessary when using immersion objectives.

The other illustration (Plate 42) is of very high magnification, viz. 5000 diameters. It was obtained by using a $\frac{1}{12}$ "immersion objective together with a high eyepiece and long camera extension; but it is doubtful if any advantage is to be obtained by carrying direct magnification to such an extent, and in many cases a sharper print can be obtained by enlargement from a negative taken at 1000 or 1500 diameters (cp. Plate 43, Fig. C).

Surirella gemma (Plate 43, Fig. A). It is difficult to resolve the secondary markings in this diatom unless oblique light is used. If the specimen is mounted in realgar the dots can be seen with axial light, but there is not sufficient contrast to obtain a satisfactory photomicrograph, hence the need of oblique illumination. 



\section{Diatoms.}

A Arachnoidiscus ehrenberghii $\times 375$

B Orthoneis splendida $\times 620$

$\begin{array}{llll}\text { Objective } & \ldots & \ldots & \text { Zeiss } 16 \mathrm{~mm} \text {. Apo. N.A. } 0.3 . \\ \text { Ocular } & \ldots & \ldots & \text { Zeiss Compensating, } 12 . \\ \text { Condenser } & \ldots & \text { Watson's Universal Achromatic. } \\ \text { Illuminant } & \ldots & \text { Liliput } 4 \text { amp. arc. } \\ \text { Aux. Condenser } & \text { Double convex lens, giving parallel beam. } \\ \text { Screen } \quad \ldots & \ldots & \text { Wratten's B (green). } \\ \text { Plate } \quad \ldots & \ldots & \text { Imperial N.F. } \\ \text { Exposures } & \ldots & 3 \text { sec. and } 7 \text { sec. }\end{array}$

These are examples of diatoms of moderate thickness, for which it is necessary to employ a low-power objective to give covering power, and combined with a high eye-piece or long extension to give sufficient magnification to show all the detail. The use of a large aperture to resolve fine secondary detail is of less importance. 

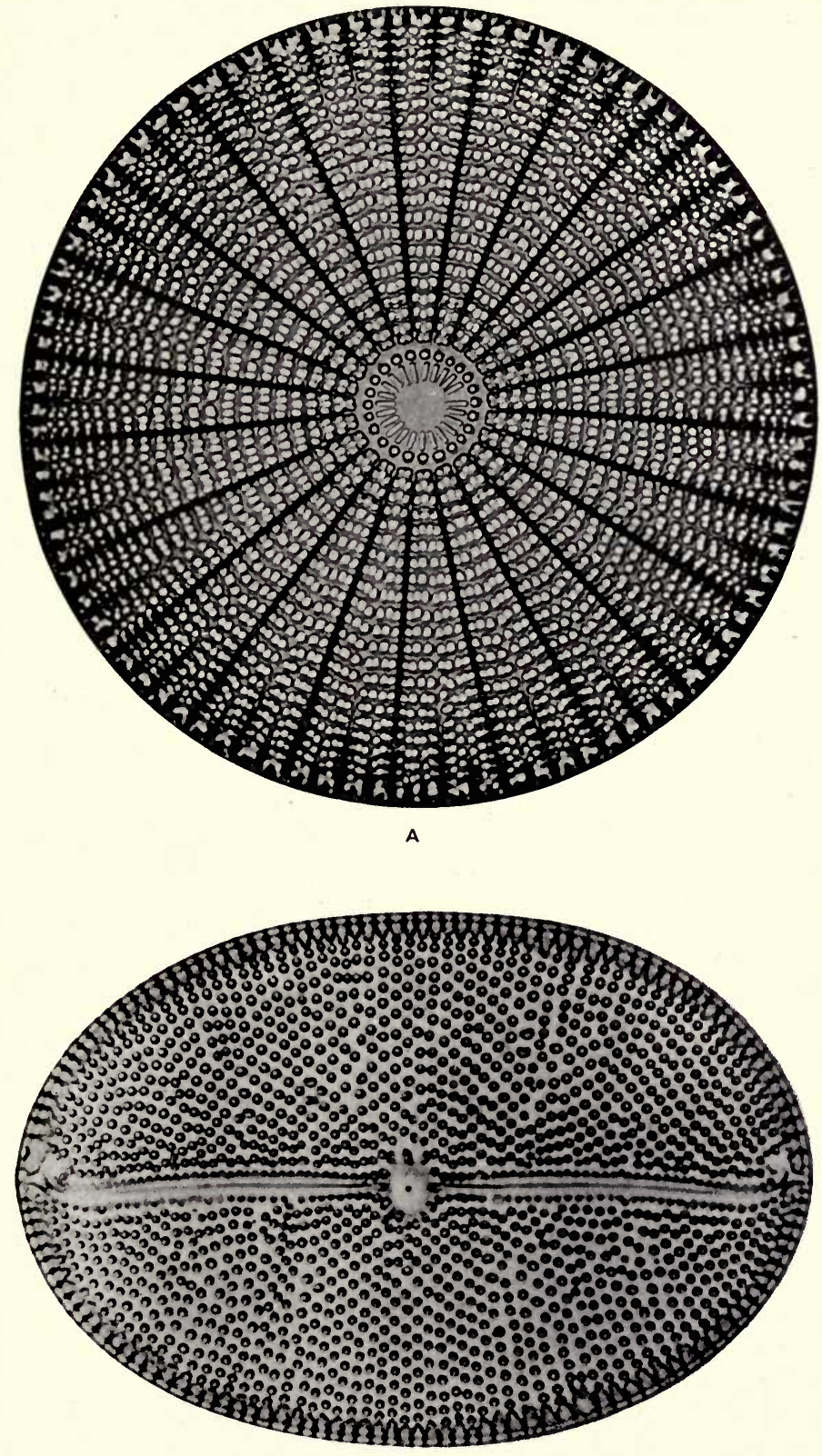




\title{
Comparison of the resolution produced by objectives of high and low N.A.
}

\author{
Suitable eye-pieces and camera extension were used to give \\ equal magnifications.
}

Dıатом-Triceratium favus $\times 620$

A

Objective $\ldots \quad \ldots \quad$ Zeiss Apo., $16 \mathrm{~mm}$.

N.A

Ocular

Condenser

Illuminant

Aux. Condenser

Screen $\quad \ldots . . .$.

Plate

Exposure .......

Developer

$$
0.30 \text {. }
$$

Zeiss Comp. $\times 12$.

Holos, top lens removed.

4 amp. arc.

Biconvex lens, parallel light.

Wratten's D+H. Violet light, 4200-4600.

Imperial N.F.

30 sec.

$5 \mathrm{sec}$.
B 0.95 .

Zeiss projection $\times 4$. Holos, stopped down to N.A. 0.8 .

The magnification produced by the $16 \mathrm{~mm}$. objective and $\times 12$ ocular is "empty," in that no more detail is visible than could easily be seen at a much less magnification. The objective of high N.A. gives a much greater resolution, and reveals detail that can only be properly seen at a comparatively large magnification. Its penetration is low, and only a small portion of the diatom can be focussed and resolved at one time.

The $\mathrm{D}+\mathrm{H}$ screens giving monochromatic violet light were used for resolution; they also diminished the excessive brightness of the image on the ground glass and made focussing easier. 


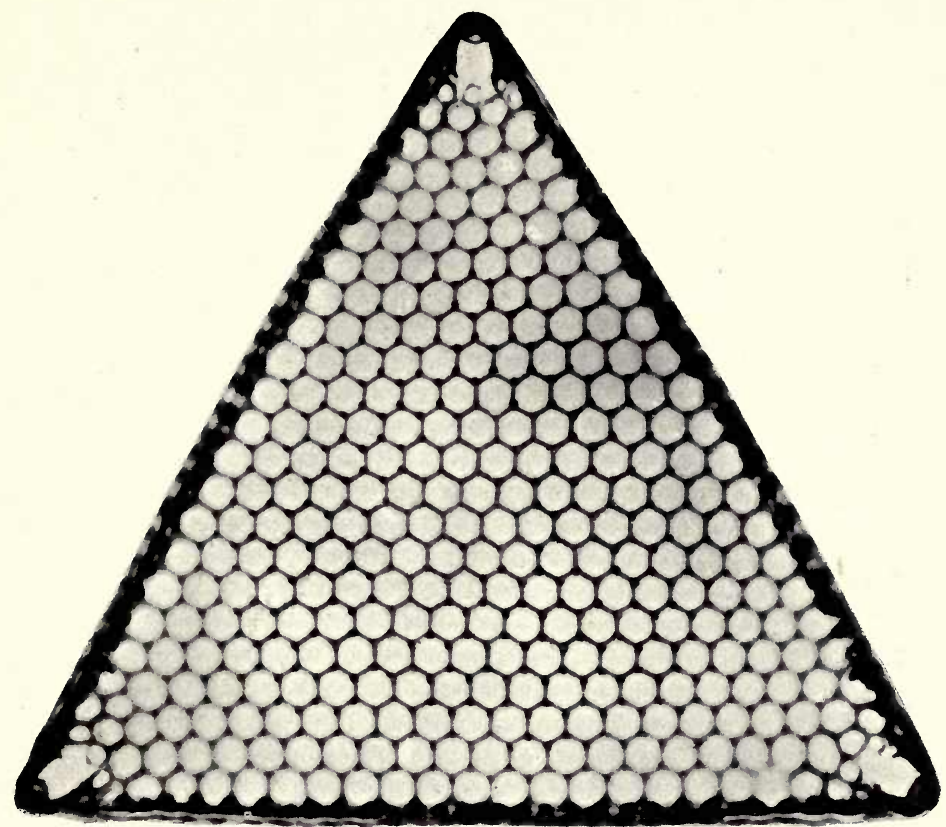

A

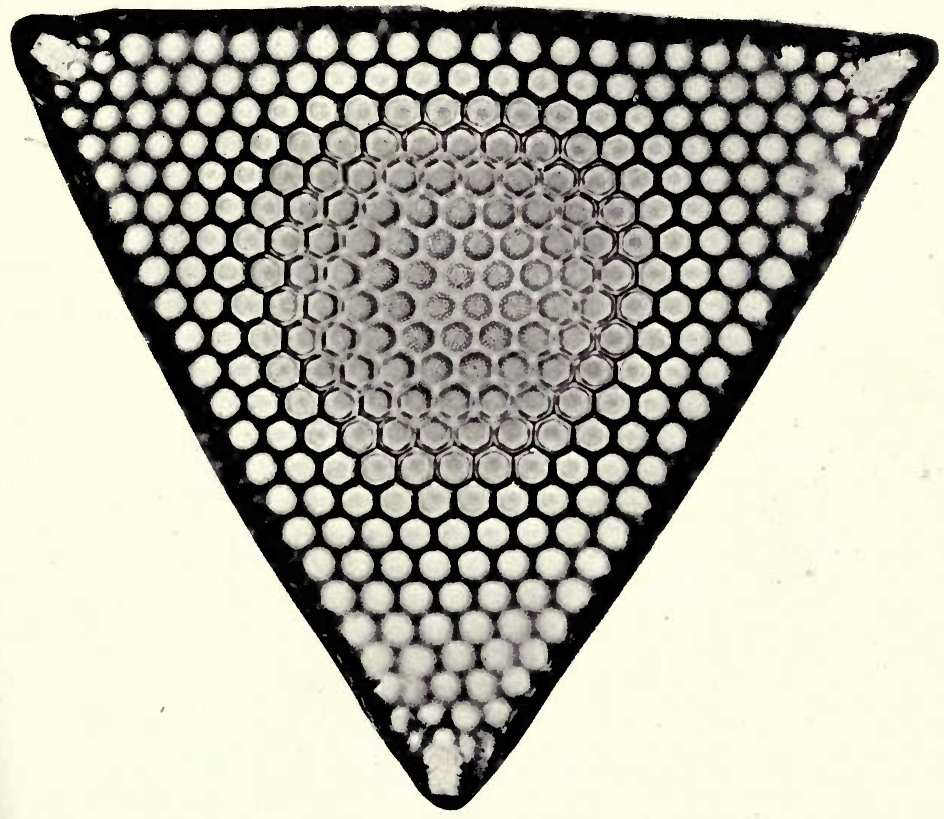






\section{Diatoms.}

A Actinoptychus heliopelta $\times 270$

B Part of the same Diatom $\times 1250$

A

B

Objective ... ... Leitz No. 3. $\frac{23^{\prime \prime}}{3} \quad$ Leitz $\frac{1}{10} "$ Achromatic, oil immersion.

Ocular $\quad \ldots \quad \ldots$

Condenser $\quad$...

Illuminant ...

Aux. Condenser

Screen $\quad \ldots \quad \ldots$

Plate $\quad \ldots \quad \ldots$

Exposure ... ...
Watson's No. $2 \times 6$.

Watson's Universal Achromatic.

Liliput 4 amp. arc.

Double convex lens, giving parallel light.

Green. Wratten's B.

Imperial Process. Wellington's Anti-

Screen.

$10 \mathrm{sec}$.

A gives the general appearance of the diatom, but does not exhibit any secondary structure. The condenser was stopped down to give depth of focus, as this diatom is comparatively thick.

B The secondary structure dots are shown in the primary hexagonal areolations. These can be easily made out with an immersion objective of $\frac{1}{10}$ " or $\frac{1}{12}$ " focus, and no difficulty should be experienced in demonstrating them with a good $\frac{1}{8} "$ or $\frac{1}{6}$ ".

\section{Pleurosigma angulatum $\times 720$}

Baker $\frac{1}{12} "$ Achromatic oil immersion objective. Leitz No. 4 ocular. Abbe condenser. Liliput arc lamp. Bull's-eye condenser. Green screen, Wratten's B. Imperial process plate. Exposure $40 \mathrm{sec}$.

This photomicrograph was taken with the simple box camera described on page 21 and Fig. 9, to show that with care satisfactory results can be obtained with the simplest apparatus.

D Navicula lyra $\times 1035$

Leitz $2 \mathrm{~mm}$. Apochromatic, oii immersion, N.A. 1.32. Zeiss compensating ocular $\times 4$. Watson's Universal Achromatic condenser N.A. 1.0. Incandescent gas. No auxiliary condenser. No screen. Imperial process plate. Exposure, $10 \mathrm{~min}$.

This photomicrograph shows the "white dot resolution"; by a slight alteration in the fine adjustment the dots appear black. The white dots provide a good test for colour correction. There is a slight colour with achromats, but the dots must be brilliant and sharply defined. 


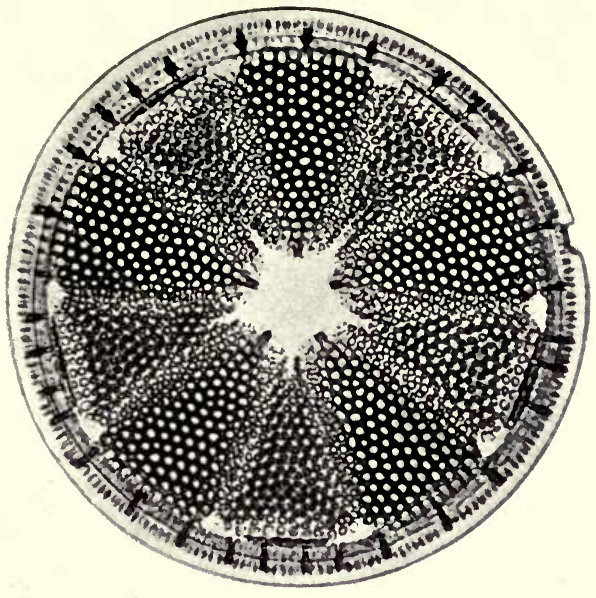

A

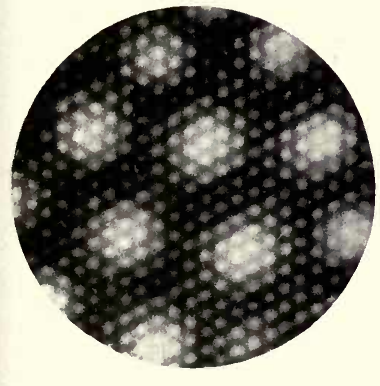

B

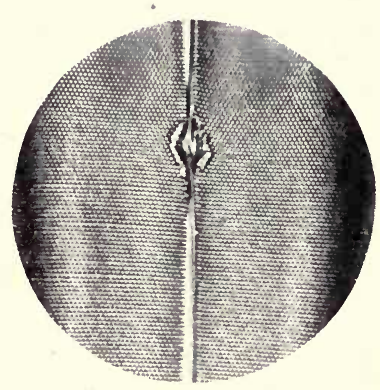

C

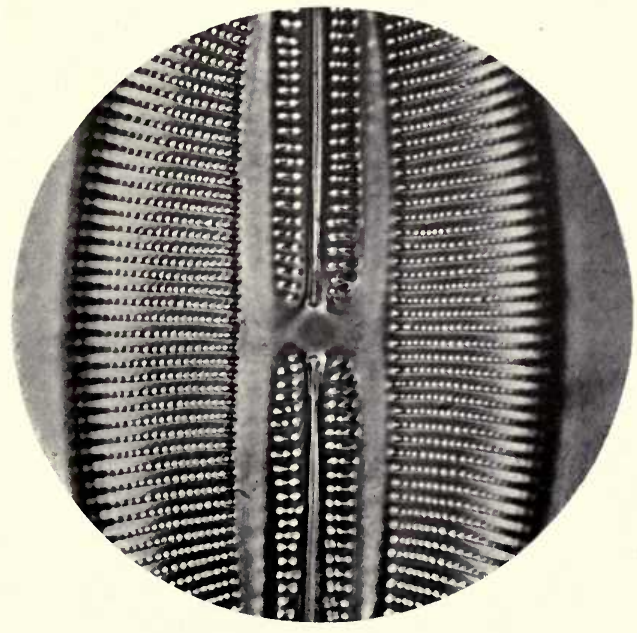






\section{Diatom.}

Pleurosigma angulatum $\times 5000$. Mounted in realgar.

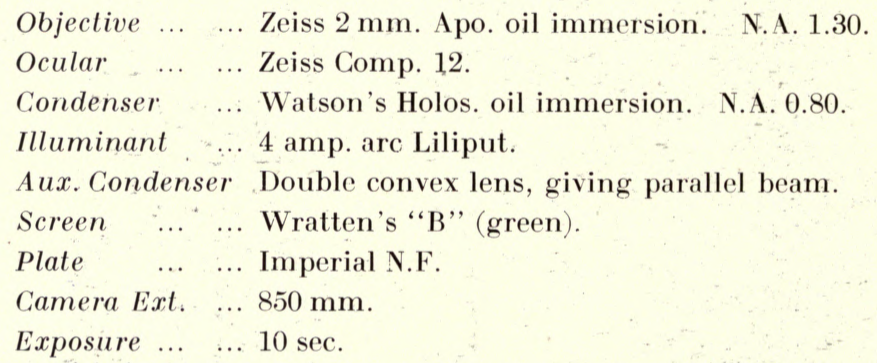

The resolution shown here is called the "white dot"; by a slight alteration of focus the dots appear black on a lighter ground.

As test object. An objective of low N.A., less than about N.A. 0.6, shows no secondary structure, or gives a resolution into crossing lines. The sharpness with which the white or black dots are revealed by an oil immersion objective is a good criterion of its quality.

An amplification of 5000 is rather unduly stretching the magnification obtainable directly from an objective. 




\section{Resolution of Diatoms by means of Oblique Illumination.}

A

Surirella gemma $\times 1620$
B

Amphipleura pellucida $\times 1250$

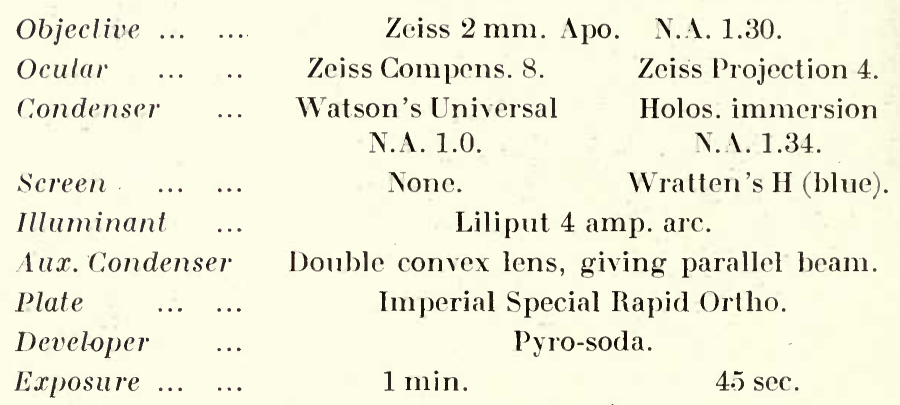

$A$ crescent or slotted stop was placed in the ring beneath the substage condenser, to direct the light obliquely on to the diatom, in a direction determined according to the principle stated on page 178. Parallel to the length of the frustule in the case of Amphipleura to show the transverse striations.

\section{Amphipleura pellucida $\times 3750$}

From a bromide enlargement of the same negative as 13 .

It is a very delicate matter to get a sharp photomicrograph at such a high magnification as this, and as a rule the simplest process is to take a negative at a lower amplification, provided that all the detail is resolved, and to enlarge it, as in this example.

The photomicrograph of $S$. gemma shows the white dot focus; a good objective reveals the dots very sharply defined. 1. pellucida may also in certain cases be resolved into dots, or, by altering the direction of the light, into longitudinal striations, but the resolution depends so much on the individual specimen that ils value as a test object is less than it would otherwise be. 

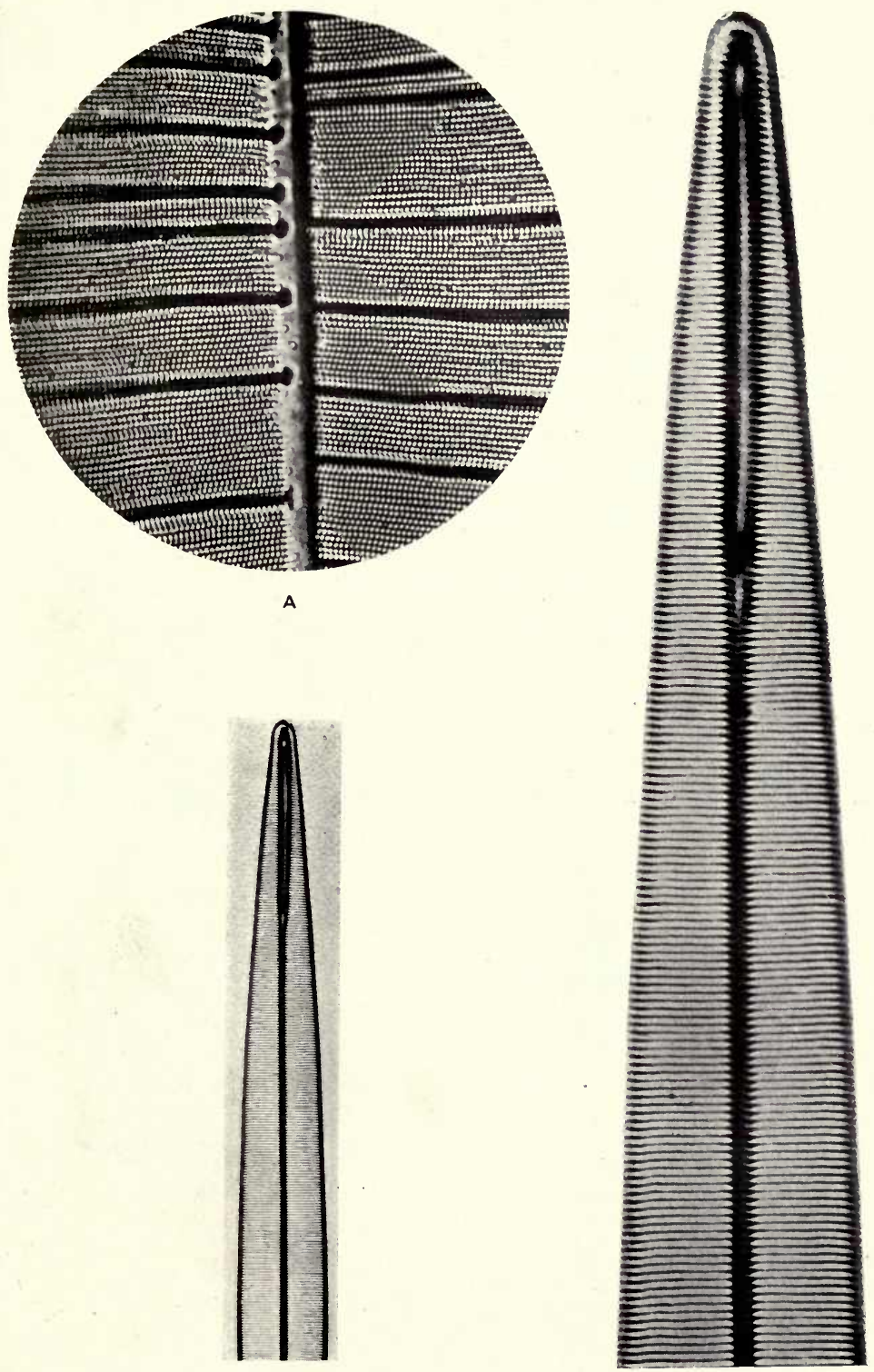

Amphipleura pellucida (Plate 43, Figs. B and C) is undoubtedly a fine test for accurate focussing; to obtain the transverse lines sharp and distinct requires patience and nicety of adjustment of condenser and illuminant. The lines can be discerned with axial illumination, but again there is lack of sufficient contrast for photography, and oblique light is required to obtain this. The slotted stop (Fig. 50) should be placed in the condenser and arranged so that light passing through the condenser impinges on the diatom in the direction of its long axis (p. 178). By carefully adjusting the stop a position will be found in which the transverse lines will be seen to the best advantage. Longitudinal striæ can sometimes be made out when the slot in the stop is at right angles to the long axis of the frustule, and it is even possible to resolve the striæ into dots with the stop placed midway between these two positions; but this requires great skill and depends to a great extent on the specimen itself, and very few permit of a distinct resolution into dots, although some present a distinctly beaded appearance in the striæ.

Exposure and development are important factors in the success or otherwise of a diatom photomicrograph; too short exposure results in a hopelessly thin negative, and the flatness produced by over-exposure is equally unsatisfactory. A developer giving good density and contrast should always be used. Pyro-soda and hydroquinone with a moderate amount of bromide are the most satisfactory. Gaslight papers give the most contrasty prints, but for fine detail glossy P.O.P. is sometimes better.

Plant and Animal Sections (Plates 5, 6 and 11, etc.).Very little need be said about the photomicrography of plant and animal sections, as the notes accompanying the plates eall attention to the chief points. An objective with a flat field is essential, and a large N.A. is very rarely 
required ; the micro-anastigmats are best for low magnifications. Monochromatic yellow-green light gives the best definition with most objectives, and should be used whenever the colour of the section permits ; the advantage of staining with a red dye is therefore obvious. For sections stained with other colours or double stained, a suitable selection of screens, as indicated on Plates 17 and 19 , is important.

Fixing the Tissues.-It is essential that both plant and animal tissues be preserved in such a manner that as little change as possible should be undergone by the constituent cells. Fixing fluids are used for this purpose, but there is no universal reagent which can be used indiscriminately for both plants and animals; different tissues require different methods of fixation in order that the best and most faithful results may be obtained. Alcohol will suffice for some of the harder stems and roots of plants, but is liable to cause a shrinkage of the cells of more delicate tissues. For these, particularly where accurate fixation of the protoplasm and nucleus of the cells is required, chromo-acetic fluid is a better reagent (p. 263). For animal tissues a useful general fixative is corrosive-acetic fluid (p. 263), but the solution employed depends greatly on the nature of the tissues to be fixed. The fixing methods are given with the formulæ in the Appendix.

Cutting the Sections.-After fixation the objects are ready for cutting and staining. Botanical preparations are usually stained after cutting, but animal tissues are as a rule stained in bulk.

Stems and roots of plants preserved in alcohol may be cut by hand with a razor, the blade of which is flooded with spirit, small or thin objects such as leaves may be placed between two pieces of elder pith, which act as a support and greatly facilitate cutting. A Catheart or other simple form of microtome enables one to obtain 
more complete sections. Animal and delicate plant tissues must be imbedded in paraffin to enable thin sections to be made. The paraffin wax employed should have a melting-point of from $45^{\circ}$ to $52^{\circ} \mathrm{C}$., and be kept melted in a water bath, the temperature of which should not be allowed to rise more than one or two degrees above the melting-point of the wax.

Imbedding in Paraffin.-The material, hitherto preserved in $70 \%$ alcohol, is dehydrated by washing in $90 \%$ alcohol and transferring to absolute alcohol for a period varying from 1 to 12 hours according to its size, the alcohol being changed once or twice in the meantime. It is then placed in cedar oil or xylene until translucent, then immersed in melted paraffin until thoroughly impregnated. This may take from 1 to 6 hours. The object is transferred to a watch-glass and melted wax poured over it, and then rapidly cooled by floating on water. When the wax is quite cold it is ready for cutting.

An alternative method that is generally adopted for plant material is as follows :-

The dehydrated material is placed in a mixture of absolute alcohol and chloroform, and then in pure chloroform until cleared. Then placed in a saturated solution of paraffin in chloroform for some hours, afterwards transferred to melted wax until all traces of chloroform have been driven off and the object is thoroughly impregnated with wax, finally imbedded in a watch-glass as previously described.

Cutting and Mounting Sections of Imbedded Material.A microtome is necessary for cutting imbedded sections ; very good results can be obtained with one of the less expensive kinds such as Cathcart's, but for serial sections one of the more elaborate forms, such as the Minot or the Cambridge rocking microtome, is essential. For large objects, as, for example, longitudinal sections 
of the mouse (Plate 15), a sliding microtome of the Thoma pattern is necessary.

To mount the section, a carefully cleaned slide is flooded with water and the section floated on with a fine brush. The slide is gently warmed until the section flattens out and all creases disappear. After cooling, the slide is tilted and the water drained off, the section carefully arranged and allowed to dry; this process may be hastened by placing the slide in a water oven at a lower temperature than the melting-point of the wax. When quite dry the section will adhere tenaciously to the slide, if the latter was perfectly clean.

The wax is removed by immersing the slide in xylene. If the material was stained in bulk and does not require double staining a drop of Canada balsam is placed on the section and a cover-glass gently lowered on to it.

Staining Sections on the Slide.-After removal from xylene the slide is placed in absolute alcohol to wash out the xylene, then washed in $90 \%$ alcohol, and, if an aqueous stain is to be used, the slide is passed through a graded series of alcohols $(70 \%, 50 \%, 30 \%)$ to water; then immersed in the stain for the requisite time, washed in water and taken through the graded alcohols in ascending series until absolute alcohol is reached, cleared in xylene and mounted in Canada balsam. If the stain to be used is an alcoholic solution it may be unnecessary to pass the slide through alcohol of lower strength than $70 \%$. Passage through graded alcohols must never be omitted when sections are transferred from alcohol to water or vice versa, and the steps between the various grades must not be too great, or the diffusion currents set up will be liable to damage delicate preparations.

Staining the Preparations.- The following is an outline of the processes used for the preparations of which photomicrographs are given.

Small Objects Mounted Entire.-Embryo Chick (Plate 
11). Fixed in Perenyi's fluid, a very useful fixing agent for embryological work. Stained in Mayer's carmalum for a few minutes, washed in water to remove alum, dehydrated and mounted in balsam. It is often advisable to dilute the stain with water and leave the object in it for a longer time. This is a particularly good stain for Polyzoa, Hydrozoa, larval echinoderms, and other small objects.

Double Staining Sections.- Sections of stems, roots, and leaves are double stained to differentiate between those cell-walls which consist of pure cellulose, and others that have been modified by lignification. The method depends on the selective affinity of some dyes for cellulose, and of others for lignified tissues.

Clematis vitalba. Transverse section of stem (Plate 17, Figs. A, B, and C).- Stained by immersion in a single solution, picro-aniline blue. Cellulose walls become blue, and lignified tissues yellow.

Cycas revoluta. Transverse section of Petiole (Plate 6).-Stained in Ehrlich's hæmatoxylin and counterstained in safranin. Hæmatoxylin stains cellulose but not lignified tissues, the safranin stains the latter red. Immerse the section in Ehrlich's hæmatoxylin for a few minutes, wash out excess of stain in $70 \%$ alcohol. Wash in tap water, the bluish-purple colour is converted to deep blue. Counterstain by immersion for a few minutes in alcoholic solution of safranin, wash out excess of colour in $95 \%$ alcohol, dehydrate in absolute alcohol ; clear in xylene and mount in Canada balsam.

Staining in Bulk and Counterstaining Sections.-Most animal tissues, and such plant structures as root apices and flower and leaf buds, are stained in bulk prior to imbedding and cutting the sections. The sections after fixing on the slide may be counterstained in a coal-tar dye or picric acid.

Dividing Nuclei of Plant and Animal Tissues (Plate 
44, Figs. A and B).-The root apex of Fritillaria was fixed in Hermann's fluid. The developing egg of Ascaris in acetic alcohol. These two reagents are valuable for cytological work, particularly for karyokinesis.

Both were stained in Heidenhain's iron hæmatoxylin according to the procedure given on page 265. This stains the chromatin sharply. The cytoplasm was counterstained in eosin (Fig. A), or in orange G. (Fig. B).

Sections of Mouse (Plates 15 and 30).-Fixed in Perenyi's fluid. Stained in bulk in borax carmine (p. 264), the sections counterstained in aniline blue. The colours intensified and further differential staining obtained by washing in picric acid, dissolved in alcohol or in the clearing xylene. 



\section{Medium and High-power Photomicrography of moderately thick sections.}

A Fritillaria imperialis, Longitu dinal Section of Root $\times 420$, showing nuclear division.

B Section through Fertilized Egg of Ascaris megalocephala $\times 1200$ First cleavage after fertilization.

A

B

Objective ... ... Leitz $\frac{1}{10}$ " oil immersion. N.A. 1.30.

Ocular $\quad . . \quad \ldots \quad$ None. Zeiss projection $\times 4$.

Condenser ... Watson's Holos. immersion. Stopped down to N.A. 0.8 .

Illuminant $\quad$... Liliput 4 amp. arc.

Aux. Condenser Double convex lens, adjusted for parallel light.

Screens .... ... Wratten's B (green) B (green). and $\mathbf{H}$ (blue).

\begin{tabular}{lllll} 
Plate & $\ldots$ & $\ldots$ & \multicolumn{2}{c}{ Imperial process. } \\
Exposure & $\ldots$ & $\ldots$ & $1 \mathrm{~min}$.
\end{tabular}

A Several stages in nuclear division can be made out in this figure. Nuclei with the chromatin in a reticulum, others in which the chromosomes are differentiated; an equatorial stage where the chromosomes are grouped in the middle of the cell, and also stages at the completion of nuclear division in which the new cell wall can be seen separating the daughter nuclei.

B The chromosomes are arranged at the equator, the nuclear spindle is shown, also the centrosomes at the poles.

Both these sections were a trifle thick, but sufficient depth of focus was obtained by restricting the aperture of the condenser. It is generally advantageous to use a lower power objective with smaller N.A. and increase the camera extension until the necessary magnification is obtained, when considerable penetration is required. 


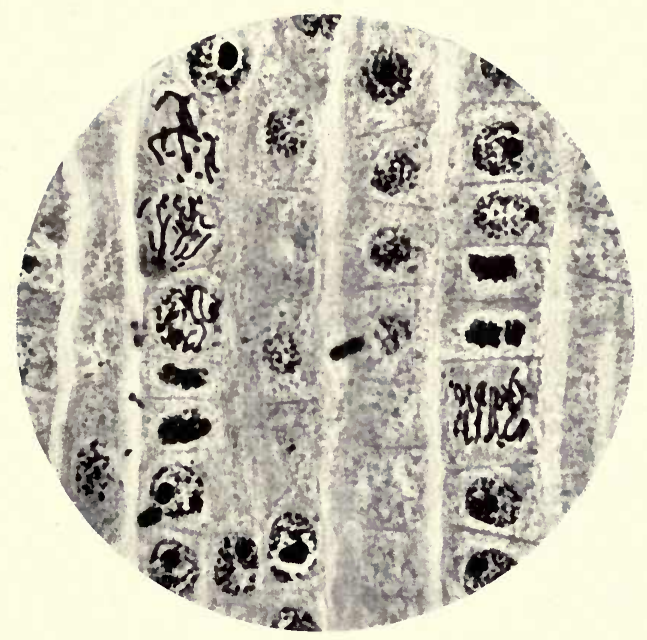

A

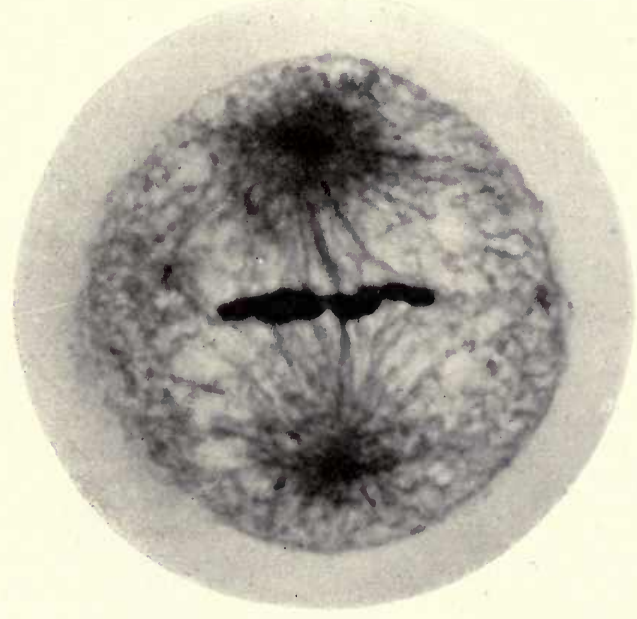





\section{APPENDIX OF FORMULA, ETC.}

\section{FIXING AND HARDENING REAGENTS}

Chromo-Acetic Acid Solution.

Chromic acid ................ 1 gram.

Glacial acetic acid............... .5 c.c.

Water ..................... $98 \cdot 5$,

This solution is a good all-round fixative for plant material. It can be used full strength for stems, leaves, buds, etc., but must be diluted with equal quantities of water, or even more for delicate objects such as algæ. The length of time for which the material should remain in the fluid depends on the size of the object, in any case it should be several hours.

After fixing, the objects are placed in a muslin bag and washed in running water for 6 hours, after which they are passed through graded alcohols, $30 \%, 50 \%, 70 \%$ to $90 \%$.

Corrosive-Acetic Fuuid.

Saturated solution of corrosive sublimate ...100 c.c. Glacial acetic acid..................5 c.c.

After fixation wash in $70 \%$ alcohol to which a few drops of tincture of iodine have been added, then wash in $70 \%$ alcohol until all colour of iodine has been removed. Objects can then be kept in this strength of alcohol until required for use.

Perenyi's Fluid (Chromo-nitric Acid).

Alcohol $(92 \%)$..................

Nitric acid $(10 \%) \ldots \ldots \ldots \ldots \ldots \ldots . . . .4$,

Chromic acid $(0.5 \%) \ldots \ldots \ldots \ldots \ldots . . . .$.

Objects are fixed in this fluid for 5 or 6 hours, then treated with $70 \%$ alcohol for 2 days, changing the alcohol frequently, passed on to $92 \%$ alcohol for some days, and finally treated with absolute alcohol for 2 to 3 days.

Hermann's Fluid (Platino-aceto-osmic Acid).

Platinic chloride $1 \%$ solution .......15 parts

Osmic acid $2 \%$ solution $\ldots . \ldots \ldots \ldots 4$,

Glacial acetic acid.............. 1 part 
Flemming's Fluid (Chromo-aceto-osmic Acid).

Chromic acid $1 \%$ solution ........25 parts

Osmic acid $1 \% \quad, \quad \ldots \ldots \ldots \ldots 10$,

Acetic acid $1 \% \quad, \quad \ldots \ldots \ldots \ldots 10$,

Water.........................,

This is a cheaper fluid than Hermann's, but equally useful.

Neither of these fluids penetrates very well, consequently the tissues to be fixed should be cut into very small pieces. Objects may remain in these fluids for several hours or even days; they are afterwards washed in running water for 6 hours and passed through graded alcohols.

\section{ACetic Alcohol.}

Absolute alcohol .............6 parts

Glacial acetic acid.............. part

Chloroform .................... parts

This is very useful in such cases as the segmenting eggs of Ascaris, which are covered with a shell that resists the passage of fixing fluids such as Hermann's or Flemming's.

After fixing, the objects are washed in strong alcohol (95\%) until all traces of smell of acetic acid have disappeared.

\section{STAINING REAGENTS}

Borax-Carmine (Grenacher).

To a $4 \%$ solution of borax in water add $3 \%$ of carmine and boil, to this add an equal quantity of $70 \%$ alcohol, leave standing for 2 days and filter.

Objects to be stained in bulk should be left in the stain until thoroughly penetrated, from 2 hours to several days, according to size. They are then placed in acid alcohol (alcohol 70\% 100 c.c., hydrochloric acid 4 drops) and left in this until they have a bright red translucent appearance, they are then washed in $70 \%$ alcohol to remove all traces of acid.

For staining sections this fluid is not so satisfactory as Carmalum.

Carmalum (Mayer).

Carminic acid ............. 1 gram.

Alum ................... 10,

Hot distilled water .............200 c.c.

To the solution add a few drops of thymol as a preservative. The material after staining must be washed in water prior to placing in alcohol, otherwise crystals of alum are liable to be deposited. 
EhrLich's ACID Hæmatoxylin.

Water...................... 100 c.c.

Absolute alcohol .................100,

Glycerin ..................100 ,

Glacial acetic acid............. 10,

Hæmatoxylin................ 2 grammes

Alum in excess

This mixture must be left in the light to ripen, which will take some weeks or even months; it gradually acquires a dark red colour.

Sections are stained in this fluid in a few minutes.

Material in bulk should remain in the stain from 2 hours to 2 or 3 days, afterwards washing in $70 \%$ alcohol.

\section{Heidenhain's Iron Hamatoxylin.}

Solution A-

Iron alum ................ 4 grammes

Distilled water..............100 c.c.

Solution B-

Hæmatoxylin crystals ......... 5 gram.

Distilled water ............... 100 c.c.

Sections are placed in A for from half an hour to two hours, then washed in tap water and transferred to B for half an hour. They are then rinsed in tap water and again placed in $\mathbf{A}$. The stain is gradually washed out of the sections, and the progress of differentiation should be carefully observed under the microscope. When the stain has been washed out of all parts of the section except the chromatin of the nuclei the slide should be washed in running water for 10 minutes, counterstained, dehydrated, and mounted.

\section{Picro-Aniline Blue.}

To a saturated solution of picric acid in $92 \%$ alcohol add aniline blue until the liquid becomes deep blue-green in colour.

Aniline Blue.-Counterstain to Borax-carmine.

Aniline blue ............... $\cdot 2$ gram.

Alcohol $(92 \%)$................ 100 c.c.

Safranin.-Counterstain for Hæmatoxylin.

Saturated solution in $92 \%$ alcohol.

This can be diluted with an equal quantity of water. 
Eosin.-After Hæmatoxylin (animal tissues).

Eosin................. 1 gram.

Alcohol $(92 \%) \quad \ldots \ldots \ldots \ldots \ldots \ldots$ c.c.

Orange G.-After Heidenhain's Iron Hæmatoxylin and sometimes after Ehrlich's Hæmatoxylin.

It is a precise plasma stain.

Saturated solution in water.

Iodine Green.-Counterstain to Carmalum, stains lignified tissues in plant sections.

Iodine green............. 1 gram.

Alcohol $(92 \%) \quad \ldots \ldots \ldots \ldots \ldots \ldots$...........

Picric Acid.-After Borax-Carmine.

Saturated solution in alcohol.

As the picric acid dissolves out in alcohol it is sometimes advisable to add it to the xylene used for final clearing before mounting.

Note.-Industrial methylated spirit may be used in place of $92 \%$ alcohol.

\section{Stains FOR BaCteria}

Carbol-Fuchsine.-

Fuchsine .................. 1 part

Absolute alcohol ................ 10 parts

$5 \%$ aqueous solution of phenol (carbolic

acid) $\ldots \ldots \ldots \ldots \ldots \ldots \ldots \ldots \ldots \ldots \ldots \ldots \ldots \ldots \ldots \ldots$,

LöffleR's Methylene Blue.-

Caustic potash solution ( 1 in 10,000 ) ....100 parts

Saturated alcoholic solution of methylene

blue ..................... 30 ,

Gram's Method.-

A. Saturated alcoholic solution of Gentian

violet................... 11 parts

Saturated aqueous solution of anilin oil ..100 ,

Mix and filter.

B. Iodine 1 part. Potassium iodide 2 parts. Water 300 parts.

C. Saturated aqueous solution Bismark brown. 
Transfer preparation from alcohol to A for 1 to 3 minutes. Wash in alcohol, place in B for 3 minutes. Wash in alcohol, place in $\mathrm{C}$ for $\mathbf{5}$ minutes. Dehydrate and clear as usual.

In a section of animal tissues this stains most bacteria while clearing the stain from the nuclei.

\section{Double Stain for Sporulating Bacteria}

Stain in hot carbol-fuchsine for 5 minutes. Decolourise in $5 \%$ acetic acid. Wash in water.

Stain in Löffler's methylene blue. Spores remain red, bacteria blue.

\section{Contrast Screens for Various Stains}

The following table from Wratten and Wainwright's Photomicrography gives the screens of the " $\mathrm{M}$ " series most suitable for use with various stains.

\begin{tabular}{|c|c|c|c|}
\hline Stain. & Spectral Absorption Bands. & Screens. & $\begin{array}{l}\text { Band } \\
\text { used. }\end{array}$ \\
\hline nil & 5 & $\& \mathrm{E}$ & -60 \\
\hline & Gener & & \\
\hline o $R_{e}$ & & & $46-51$ \\
\hline & & & $51-54$ \\
\hline $\cos$ & & & $1-5$ \\
\hline & & & $51-$ \\
\hline Ger & & & $56-6$ \\
\hline $\begin{array}{l}\text { Hæms } \\
(\mathrm{Kl}\end{array}$ & $\begin{array}{l}\text { Gradual from Violet } \\
\text { to } 5800\end{array}$ & $B \& C$ & $46-51$ \\
\hline (Ehr & Gradual th & & 51 \\
\hline & & & \\
\hline d & & & $6]$ \\
\hline ei & Ge & & $51-$ \\
\hline & & & \\
\hline & & $B \& E$ & $56-6$ \\
\hline & & & \\
\hline Pic & $51-53$ & & $51-5$ \\
\hline Rose & & & $51-54$ \\
\hline
\end{tabular}

For insects and yellow sections generally, photographFor contrast, with a $C$ Screen ;

For detail in the section, with an F Screen. 
NARCotising Solution for Rotifers (C. F. Rousselet, F.R.M.S.).

$$
\begin{aligned}
& 2 \% \text { solution hydrochlorate of cocaine } \ldots 3 \text { parts } \\
& \text { Alcohol (or methylated spirit) } \ldots \ldots \ldots \ldots .1 \text { part } \\
& \text { Water } \ldots \ldots \ldots \ldots \ldots \ldots \ldots \ldots \ldots \ldots \\
& \text { parts }
\end{aligned}
$$

\section{PHOTOGRAPHIC FORIMULE}

Formule for almost every photographic process are to be found in the British Journal Photographic Almanac, which is an almost indispensable book of reference for photographers. Several of the following formulæ are from that source.

\section{Developers}

Metol-Quinol (R. L. Boyd).-Concentrated stock solution :-

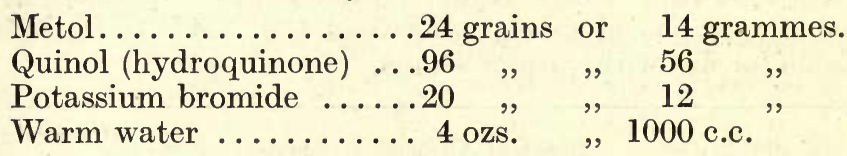

When dissolved add sodium sulphite (crystals crushed small) $1 \frac{1}{2}$ ozs. ( 400 grammes). To the white pasty mass add sodium hydrate (caustic soda) 64 grains (40 grammes).

For Plates and Gaslight Papers.-Take 1 part of the stock solution and add 7 parts of water.

For Bromide Papers and Lantern Slides.-Add 15 parts of water to 1 part stock solution. Add 2 drops per oz. of $10 \%$ potassium bromide solntion for papers and lantern slides.

Watkins Factor, 16.

Time of Development.-At $55^{\circ}$ F., $3 \frac{3}{4}$ minutes; at $60^{\circ}$, 3 minutes; at $65^{\circ}, 2 \frac{1}{2}$ minutes. One oz. of the developer contains metol .75 grains, quinol 3 grains, sodium sulphite (cryst.) 20 grains, caustic soda 2 grains, potassium bromide $0 \cdot 6$ grains.

Hydroquinone.

A. Hydroquinone .....160 grains or Sodium sulphite .... 2 ozs. ", Citric acid .......6 60 grains ", Potassium bromide .. 40 ", ", Water to ....... 20 ozs.

B. Caustic soda........160 grains ", Water to.........20 ozs.

20 grammes 100 8
5

20 grammes. 1000 c.c. 
For Plates.-Use A 1 oz., B 1 oz., water 2 ozs.

Watkins Factor, 6.

Pyro Soda (Ilford Formula).

A. Sodium carbonate (soda crystals) 2 ozs. or 100 grms. Sodium sulphite (crystals) ...2, , , 100 ,, Potassium bromide .......20 grains , 2 , Water up to ...........20 ozs. , , 1000 c.c.

B. Pyro ............. 1 oz. , 50 grms. Water up to...........6 ozs. , 300 c.c.

Nitric acid ............20 drops

For Plates.-Take one part of B, make up to 10 parts, and add 10 parts of $A$.

B keeps fairly well, but it is preferable to weigh out the pyro required each time as follows :-

A $1 \mathrm{oz}$, water $1 \mathrm{oz}$, pyro 2 grains to 4 grains.

Watkins Factor, 7 (6 for negatives of ordinary contrast).

Time of Development with 3 grains pyro per oz.:$55^{\circ}$ F., 6 mins. ; $60^{\circ}, 5$ mins. ; $65^{\circ}, 4$ mins.

Developers in Concentrated Solution.-Such as Azol, Certinal, Rodinal, and Victol should be diluted for use according to the instructions supplied with them. They are somewhat as follows :-

For Plates.-Developer 1 part, water 20 parts.

Bromide Papers and Fast Lantern Plates.-Developer 1 part, water 30 parts.

Gaslight Papers and Slow Lantern Plates.-Developer 1 part, water 15 parts. 2 drops $10 \%$ potassium bromide may be added to each oz. of solution for papers or lantern slides, or even for plates if desired.

Watkins Factor, 40.

Time of Development.-At $55^{\circ}$ F., 8 mins. ; $60^{\circ}, 7$ mins. ; $65^{\circ}, 6$ mins.

Tank Development. - 1 part developer, 100 parts water.

Time for Tank Development. $-55^{\circ}$ F., 30 mins. ; $60^{\circ}$, 25 mins. ; $65^{\circ}, 20$ mins.

The times required for time and tank development vary with the plate, and those given may need reduction by about $\frac{1}{2}$ or increase even up to double the time given for some plates. 


\section{Colour Sensitisers for Plates}

Non-colour sensitive plates are made sensitive to the entire spectrum by bathing in the following solution :-

Pinacyanol stock solution ....... 2 parts.

Pinachrome $", \quad \ldots . . . .3 \%$

Distilled water.................50 ,

The stock solutions contain 1 part of the dye dissolved in 1000 parts alcohol; they can be bought ready prepared, and keep well. The dilute solutions do not keep, and should be mixed immediately before use, and not used more than twice.

Bathing is carried out in the same way as development, either in a dish or tank, in complete darkness or by the light of a panchromatic safelight.

Bathing in a Dish.-Immerse the plates for 3 minutes, rocking continuously.

Tank.-Dilute the above solution 20 times and leave the plates in it 20 minutes, reversing the tank once or twice.

Wash the plates in running water for 5 minutes and place on a rack to dry, preferably in a dark cupboard through which a current of dry air can be passed and kept at about $80^{\circ} \mathrm{F}$.

\section{Fixing Baths}

For negatives. Hypo (sodium

$$
\text { thiosulphate) ...4 to } 8 \text { ozs. }
$$

$$
\text { Water.......... } 20 \text {, or } 20 \text { to } 40 \%
$$

For prints.

$$
\begin{aligned}
& \text { Hypo ......... } 3 \text { ozs. } \\
& \text { Water .........20, or } 15 \%
\end{aligned}
$$

Acid Fixing Bath.-For plates, lantern slides, bromide and gaslight papers. Cleaner than plain hypo, and stainless. Hypo............. to 6 ozs. or 200 to 300 grammes Potassium meta-bisulphite .. $\frac{1}{2} \mathrm{oz} . \quad$, 25 Water...............20 ozs. " 1000 c.c.

Hypo Eliminator.-Place the plates or papers in several changes of water to which sufficient potassium permanganate solution has been added to give it a pale pink colour, until the pink colour is no longer rapidly discharged. Then rinse in water. Too strong a solution acts as a redúcer. 
Hardening Bath

Formalin ................ $1 \mathrm{oz}$.

Water ...................... 10 ozs.

Soak prints or plates for about 10 minutes and wash.

INTENSIFIERS. 1. Mercury

A. Mercuric chloride (corrosive sublimate) $\ldots \ldots \ldots 1 \mathrm{oz}$.

Hot water .....................

This will give a saturated solution; when cold pour off the solution from the deposited crystals and add 30 drops hydrochloric acid.

B. Ammonia $(\cdot 880) \ldots \ldots \ldots \ldots \ldots \ldots$ drops

Water ...................... 1 oz.

Well wash plates till free from hypo, soak in A until bleached, wash again, and blacken in B. Wash for $\frac{1}{2}$ hour in running water. Solution A may be used repeatedly.

2. Chromium (C. Welborne Piper).

Potassium bichromate...10 grains or 23 grammes

Strong hydrochloric acid .. 5 minims , $0 \cdot 3$ c.c.

Water........... $1 \mathrm{oz}$, 1000 ,

Bleach in solution, wash until free from yellow stain, and re-develop in Amidol or other non-staining developer.

Prolonged washing after fixation is not necessary with this intensifier, and the process may be repeated if greater intensification is required.

\section{REDUCERS}

1. Howard Farmer's.-Attacks thin part of image first and increases contrast.

Hypo solution ................... in 5

Add enough Potassium ferricyanide (red prussiate of potash) solution to give a pale yellow colour.

Negatives must be thoroughly soaked in water before immersion in this solution. As soon as reduction has proceeded far enough, plunge into water and wash thoroughly.

2. Persulphate.-For use with negatives that are too contrasty; attacks dense portions first.

Ammonium persulphate.....30 grains or 35 grammes

Water ............... 2 ozs.

Concentrated sulphuric acid .. 1 drop " " 18 drops

Use solution fresh. 
As soon as negative is sufficiently reduced immerse in 5\% sodium sulphite solution, then wash.

\section{Gold Toning Solution}

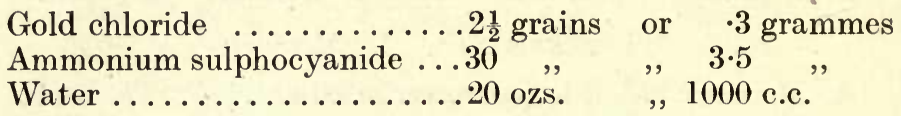

Dissolve the gold and ammonium sulphocyanide separately in half the quantity of boiling water. Then slowly add the gold solution to the other, stirring meanwhile.

If the solutions are made in cold water, allow to stand 12 hours before use.

$2 \frac{1}{2}$ grains of gold will tone 30 quarter-plate prints.

Wash prints for $\frac{1}{4}$ hour in running water before toning, keep moving in toning bath until the desired colour is obtained, wash again, fix and wash for one hour at least. Prints become warmer in tone during fixation, and some experience is required to know when to stop toning to get a desired final colour.

\section{Platinotype Developer}

Stock solution.-Potassium oxalate ... 2 ozs. or 140 grms. Water phosphate. $\frac{1}{2}$ oz. ", 35 "......

Make acid, if necessary, by adding

Oxalic acid .....66 grains or 10 grms.

For use take 1 part stock solution and 1 part water.

Fixing.-Hydrochloric acid .... 1 oz. or 12 c.c. Water ............80 ozs. , 1000 ,

Immerse prints for 5 minutes in three separate baths. Wash for 15 minutes and dry.

\section{Three-Colour Photography}

Sensitising Solution.-

Potassium bichromate .... 1 oz. or 50 grammes

Water .............20 ozs. , 1000 c.c.

Liquor ammonia $(\cdot 880) \ldots .60$ minims,, 6 ,

Blue Toning Solution for Lantern Plates.-

$10 \%$ solution ferric ammonium citrate $\ldots 2$ ozs. or 10 c.c.

$" \quad " \quad$ potassium ferricyanide $\ldots .22, \quad, \quad, 10 \%$,


Dyes for Colour Transparencies (Von Hübl).

Red Bath.-For positive from green negative.

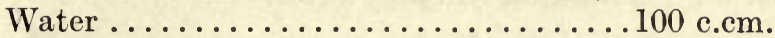

Erythrosine $(1$ in 200$) \ldots \ldots \ldots \ldots \ldots$. 5 ,

Alcohol .................... 10 ,

Blue Bath.-For positive from red negative.

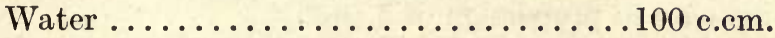

${ }^{1}$ Fast green, bluish $(1$ in 200$) \quad \ldots \ldots \ldots .20$,

Alcohol ................... 10 ,

Acetic acid ..................... 10 drops

1 F. Beyer and Co., London.

Yellow Bath.-For positive from blue negative.

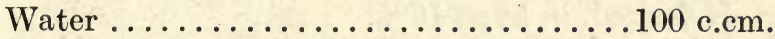

${ }^{2}$ Napthol yellow S.L. $(1$ in 200$)$....... 10 ," Alcohol .................... 10 ,

Saturated chrome alum solution ....... 5 5 Acetic acid ................ 10 drops

${ }^{2}$ Actien Gesellschaft für Anilin Fabrikation, Berlin.

\section{Screen Plate Process}

1. Development.-Rodinal, 1 in 10.

Autochromes ...........

Paget .............. , , ,

Metol-Quinol.-For Autochromes:

Metol...........32 grains or 4 grammes

Hydroquinone .....96 " " 12 "

Sodium sulphite ....4 ozs. " 200 ",

Potassium bromide...50 grains ", 6 ",

Ammonia $(\cdot 88) \ldots \ldots . \frac{1}{2} \mathrm{oz} . \quad$ " 20 c.c.

Water..........20 ozs. , , 1000 ,,

Dilute 1 part with 4 parts water and develop $2 \frac{1}{2}$ minutes at $60^{\circ} \mathrm{F}$.

For Dufay Plates.

Metol.........50 grains or 6 grammes

Hydroquinone .....17 " ", 2 ",

Sodium sulphite $\therefore \ldots 1 \frac{1}{2}$ ozs. " 75 "

Potassium bromide...17 grains ", 2 ",

Ammonia $(\cdot 88) \ldots \ldots . \frac{1}{4} \mathrm{oz}$.

Water..........20 ozs.

12 c.c.

, 1000 , 
Dilute 1 part with equal quantity of water and develop for $4 \frac{1}{2}$ minutes at $60^{\circ} \mathrm{F}$.

Wash for $\frac{1}{2}$ minute in running water.

2. Reversal.-Potassium permanganate. 2 grms. or 17 grns. Sulphuric acid ....... 10 c.c. , $\quad \frac{1}{4} \mathrm{oz}$. Water.............1000, , 20 ozs.

Or potassium bichromate may replace the potassium permanganate in proportion of 5 to 2 .

Reversal is complete in 3 or 4 minutes.

Wash 30 seconds or till stain of bichromate disappears.

3. Redevelopment.-Replace the plate in the first developer in bright light until completely blackened (3 or 4 minutes).

Wash 30 seconds.

4. Fix.-If desired, in hypo $15 \%$.

Wash $\frac{1}{4}$ of an hour.

5. Dry.-Spontaneously in air.

6. Varnish.-Gum dammar 1 part; benzole 10 parts.

Equivalent Weights of Dry and Crystalline Chemicals.Sodium sulphite, dry-

One part =two parts crystals $\mathrm{Na}_{2} \mathrm{SO}_{3} 7 \mathrm{H}_{2} \mathrm{O}$.

Sodium carbonate, dry-

106 parts $=286$ parts crystals $\mathrm{Na}_{2} \mathrm{CO}_{3}, 10 \mathrm{H}_{2} \mathrm{O}$.

(Soda crystals or washing soda.)

The quantities given in the developing formulæ refer to the crystalline chemicals. The sodium sulphite crystals may be replaced by half their weight of the dry salt, and the soda crystals by one-third the given weight if dry carbonate is used.

\section{Relation of English and Metric Weights and Measures}

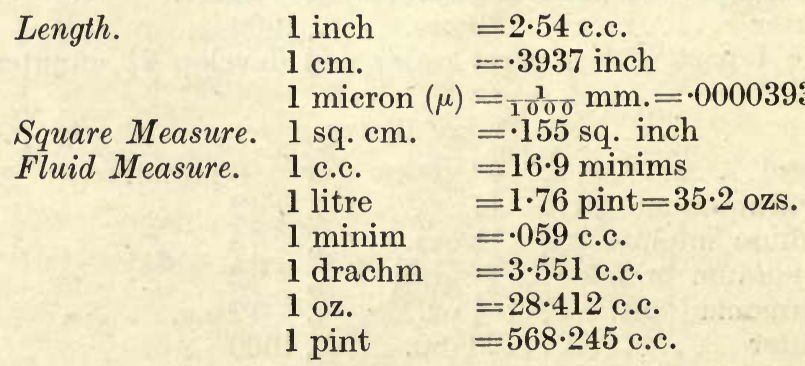



Weight.
1 gramme $=15 \cdot 432$ grains
$1 \mathrm{oz}$.
$=28 \cdot 35$ grammes
1 grain
$=\cdot 0648$ gramme

Useful Memoranda

$\begin{array}{ll}1 \mathrm{~mm} . & =\frac{1}{25} \text { inch } \\ \frac{1}{250} \text { inch } & =100 \text { micra } \\ \frac{1}{25000} \text { inch } & =1 \text { micron } \\ 5 \mathrm{~cm} . & =2 \text { inches } \\ 3 \mathrm{~mm} . & =\frac{1}{8} \text { inch } \\ 2.5 \mathrm{~mm} . & =\frac{1}{10} \text { inch } \\ 2 \mathrm{~mm} . & =\frac{1}{12} \text { inch } \\ 5 \mu & =\frac{1}{5000} \text { inch } \\ \frac{1}{100} \text { inch } & =25 \mathrm{~mm} . \\ 4 \text { litres } & =7 \text { pints } \\ 2 \text { grammes } & =31 \text { grains }\end{array}$

To convert grains per oz. to grammes per litre, multiply grains by $2 \cdot 3$.

To convert grammes per litre to grains per oz., multiply grammes by $0 \cdot 44$.

To convert c.c's. per litre to minims per oz., divide the c.c's. by 2.

\section{Enlargement Table}

\begin{tabular}{|c|c|c|c|c|c|c|c|c|c|}
\hline \multirow{2}{*}{$\begin{array}{c}\text { Focus of } \\
\text { lens } \\
\text { inches. }\end{array}$} & \multicolumn{9}{|c|}{ Times of Enlargement. } \\
\hline & 1 & 2 & 3 & 4 & 5 & 6 & 7 & 8 & \\
\hline 2 & 4 & 6 & 8 & .10 & 12 & 14 & 16 & 18 & ڤ్ \\
\hline 3 & 6 & 9 & 12 & 15 & 18 & 21 & 24 & 27 & \\
\hline 4 & 8 & 12 & 16 & 20 & 24 & 28 & 32 & 36 & $\stackrel{8}{4}$ \\
\hline 5 & 10 & 15 & 20 & 25 & 30 & 35 & 40 & 45 & $\infty$. \\
\hline 6 & 12 & 18 & 24 & 30 & 36 & 42 & 48 & 54 & 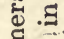 \\
\hline 7 & 14 & 21 & 28 & 35 & 42 & 49 & 56 & 63 & ह \\
\hline
\end{tabular}

The distance between lens and object for any enlargement is obtained by dividing the camera extension, as given in the table, by the enlargement.

\section{Polish for Ferrotype Plates}

Spermaceti wax ....20 grains or 45 grammes

Benzole ........1 oz. , 1000 c.c. 


\section{Aquarium Cement for Troughs}

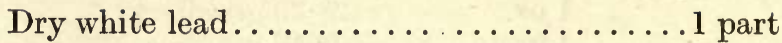

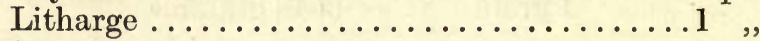

Silversand ....................

Finely powdered resin .............

Mix well and keep in a bottle. When required for use mix with boiled oil and dryers till of the consistency of putty.

Refractive Indices of Mounting Media, etc. $\mu \mathrm{D}$ Water ..................... 1.334

Glycerin ................... 1 $1 \cdot 460$

Cedar oil .................. 1·510

Oil of Cloves. . . . . . . . . . . . . . . 1.530

Canada balsam ................. 1.535

Styrax ................... 1.582

Monobromo-napthalene $\ldots \ldots \ldots \ldots \ldots 1 \cdot 658$

Realgar $\mu \mathrm{E} \quad \ldots \ldots \ldots \ldots \ldots \ldots \ldots \ldots 2 \cdot 549$

Refractive Indices of Typical Glasses, etc.

Fluor $\operatorname{spar} \mu \mathrm{D} \ldots \ldots \ldots \ldots \ldots \ldots . . \ldots 1 \cdot 433$

Crown glass of various kinds from about $1 \cdot 5$ to $1 \cdot 6$

Flint

$"$

,

,

1.54 to 1.7 , and even $1 \cdot 9$ 


\section{PHOTOMICROGRAPHIC APPARATUS}

THE following lists (I-III) of apparatus are suitable for more or less complete photomicrographic outfits, but do not include items designed for specialised work such as metallography. No. I is sufficient for most amateurs, though scarcely up to the standard required for critical photomicrography, No. II is, however, adequate for this purpose. The cost of III is necessarily very much higher than either of the others, but such an equipment is much to be desired if a large amount of critical work is contemplated. Guidance in the selection of the most suitable items for any particular ease will be found in the earlier chapters of this book. For the cost of apparatus reference should be made to the manufacturers' catalogues.

I. Student's microscope.

Objectives (achromatic) and Eyepieces. Set (iv.), p. 82.

Abbe condenser.

Camera. $-\frac{1}{4}$ or $\frac{1}{2}$ plate stand camera adapted.

Baseboard with sliding carriers.

Lamp.-Incandescent gas or Nernst.

Collector.-Adjustable bull's-eye with iris diaphragm. Colour Screens.-Set of nine $1^{\prime \prime}$ gelatine squares, or green and orange glasses.

Cooling trough.

Focussing lens.

Stage micrometer.

Stops for dark ground illumination.

II. Microscope of Group 2.

Objectives (achromatic) and Eyepieces.-Sets (i.), (ii.), or (iii.), pp. 81-82.

Condenser (achromatic).-N.A. 1.0. 
Camera. $-\frac{1}{2}$ plate, and Optical bench with focussing gear. Or a vertical camera.

Lamp.-Nernst, limelight, or incandescent gas.

Collecting lens.-Aplanatic or 4" double convex lens and iris diaphragm to fit Optical bench.

Colour Screens. Set of nine $2^{\prime \prime}$ gelatine squares.

Cooling trough.

Focussing lens.

Stops for dark ground and oblique illumination.

Davis diaphragm.

III. Microscope of Group 1.

Objectives (apochromats and achromats) with Eyepieces (p. 81).

Condensers.-Achromatic N.A. 1.0, together with a low-power illuminator and oil-immersioncondenser.

Large Camera and Optical bench completely fitted with stands for condensers, iris diaphragm and cooling trough.

Lamp.-Arc, 4-amp. Limelight. Nernst or Mercury Vapour.

Collecting Lenses.-Aplanatic condensers with iris diaphragm.

Colour Screens.-Set of nine $2^{\prime \prime}$ gelatine squares mounted in optical flats.

Cooling trough.

Stage to fit Optical bench to hold large objects.

Focussing lens.

Stops for dark ground and oblique illumination.

High-power dark ground illuminator.

Stage and Eyepiece micrometer.

Davis diaphragm. 


\section{INDEX}

Abbe coudenser, 107

- Achromatic, 108

- - and corrected condensers compared, 111

- - Numerical aperture, 107, 111

- Prof., and Apochromatic objectives, 53

- Prof., and Numerical aperture, 60

- test plate, 57

Aberration, Cliromatic, 48

- Spherical, 47

- Zonal, 51

Absorption bands, spectra, 154

- - diagranı, 159

- - of stains, 267

Acetic-alcohol fixing fluid, 264

Acetylene lamps, 96

- gas purifier, 97

Achromatic eyepiece, 74

- objective, 52

- improved objective, 54.

(See also Objective and condenser)

Actinometer for tricolour positives, 206

Actinoptychus heliopelta, 255

Adapters for dark slides, 6

Adjustment, coarse, 31

- Fine, 2. (See Fine adjustment)

Adon telephoto lens, 140

Ammoniacal copper sulphate solution, 158

Ammonium bichromate sensitising solution, 273

- persulphate reducer, 271

Amphipleura pellucida, 257

- - resolution by oblique light,
Amphipleurce pellucida test object, as, 178

Anastigmat lenses, 130

Angström units, 158

Aniline blue, 265

Animal sections, 257

Aperture. (See Numerical aperture)

Aplanatic cone, 102

- condenser, 105

- lens, 52

Apochromatic condenser, 110

- objective, 53. (See objective)

Apparatus, Sets of, 277

Applications of photomicrography, 238

Aquarium cement, 276

Arachnoidiscus ehrenberghii, 254

Arc lamp, 85

- - for alternating current, 86

- Automatic, 87

- - Baker's, 88

- - candle power, 86

- - carbons, 89

- Connections for, 89

- Current for, 85

- Enclosed, 89

- Hand feed, 88

- L Liliput, 7, 88

- Small, 86

- Zeiss' 5 ampère, 87

Ascaris, Developing egg of, 262

Autochrome plate, 210

- - development, 274

- - extra sensitised, 213

Auxiliary condenser. (Sec Condenser)

Azol, 269 
Bacillus anthracis, 242

- diphtheria, 242

- tuberculosis, 242

- typhosus, 242. (See also Bacteria) Backing plates, 219

Bacteria, Colour photographs of, 241

- Dark ground illumination of, 245

- N.A. for, 241

- Photographs of living, 243

- Photography of, 240

- Preparation of slides of, 242

- Stains for, 266

Baker's arc lamp, 88

- R.M.S. nicroscope, 41, 42

Barnard's Geometric Slide Camera, 19

Baseboard, 2, 4

- with sliding carriers, 6,8

Batteries of lenses, 81

Bausch and Lomb's large microscope, 39

- - microscope B.H., 43

- - vertical camera, 17

Beck's Imperial microscope and optical bench, 148

- London microscopes, 45

- Optical bench for enlarging, 231

- Rosenhain Metallurgical Microscope, 198

Bellows, Extra for cameras, 9

- Long extension, 9

Blue toning solution for transparencies, 273

Borax carmine, 264

Brewing, Photomicrography in, 243

Bromide papers, 228

Bull's-eye condenser, 104

Butcher's injector jet, 96

Camera, Adaptation of enlarging, 8

- - hand, 5,13

- - Reflex, 5

- - stand, 6,13

- Adaptability to vertical or horizontal position, 15

- Adjustable Vertical, 15
Camera, Barnard's, 19

- Bausch and Lomb's, 19

- Box, 21

- Convertible Britisher, 5, 13

- Enlarging, 8

- Extension and magnification, 77

- - Formula for, 131

- Long, 9

- Extra bellows for, 9

- Gordon's, 21

- Horizontal, 2 et seq.

- - and Vertical compared, 3

- Insects, For, 129

- Instantaneous, Vertical, 22, 247

- Leitz, 18

- Metallographic, 199

- Micro-metallograph, 201

- living organisms, For, 247

- Size of, 6

- Small, 21

- solid objects, For, 249

- Universal, 17

- Van Heurck, 15

- Vertical, 3, 13, 15, 22, 247

- - and Horizontal, 17, 19

- - for Opaque objects, 130

- - pillar, 16

- Watson's Laboratory, 11

- Zeiss' large, 21, 150

- - Vertical and Horizontal, 19, 134,135

Cameras, 4

Carbol fuchsine, 266

Carbon printing, 230

Carbons for arc lamps, 89

Card for focussing, 15, 19, 134, 247

Carrier for slides and oljects, 133, 136

Cedar oil, immersion fluid, 67

Cells for liquid light-filters, to make, 160

Centration of apparatus, 145

Centring nosepiece, 30

- Substage, 29

Certinal, 269

Chick in egg, 250 
Chromatic aberration, 48

- difference of magnification, $\mathbf{5 5}$

Chromo-acetic fixing fluid, 263

Chromo-isolar plates, 220

Cinemato-micrography, 187

Circle of confusion, 64

Clearing solution, 225

Clematis vitalba, 261

Coarse adjustment, 31

Colouring lautern slides, 235

Colour photomicrography, 203

- - Autochrome, 210, 273

- - Bacteria, for, 241

- Comparison of processes, 212

- Dufay, 210, 273

- - Duplicating process, 211

- - Paget, 210, 273

- - Paget duplicating, 211

- - Process, outline of, 273

- Sanger-Shepherd, 205

- - Screens for artificial lights, 204

_ - - for screen plates, 209

— - - for two-colour process, 208

- - Single process, 210

- - Solutions for, 273

- - Theory of, 204

- - Three-colour, 204

- - Two-colour, 208

Colour prints, 203

- transparencies, 203

Colour screens (or Light filters), 153

- - Carriers for, 8, 19, 138

- - Choice of, 167

- Complementary, 162

- - and contrast, 161

- - and correct colour rendering, 163

- - and definition, 161

- - and detail, 163

- Diatoms, for, 254

- Efficiency of, 154

- - factors for " $M$ " Screens, 174

$\div$ - Focussing with, 167

- - gelatine dyed, 155
Colour screens, To make, 156

- - Glass, 155

- - - circles, 157

- - illuminant and, 165

- - insects, for, 252

- - liquid, 157

- - materials of, 155

- - resolution, for, $61,161,254$

- - Rules for use of, 164

- - Stains, for various, 267

- - Sets of, 156

- - Use of, 161

Colour sensitive plates, 165, 214

- - - Sensitiser for, 270

Compensating Eyepiece, 72

- - with high-power achromats, 74

Condenser, 102

- Aplanatic cone of, 102

- colour fringes, 103

- Experiments with, 115

- Focal length of, 103

- Function of, 102

- N.A. of, 103

- Power of, 103

- Uncorrected, 103

Condenser, Auxiliary (or collecting lens), 102

- - Aperture of, 125

- Aplanatic, 105

- Biconvex lens, 106

- - Bull's-eye, 104

- - To centre, $147,148,152$

- Comparison of corrected and uncorrected, 105, 116

- - colour fringes, 116, 126

- Corrected, 105

- Flasks as, 93, 106

- - focus, To, 147

- - Iris diaphragm for, 106

- K Köhler, 105, 134

- Mounts for, 8, 106

- - negative lens, Use of, 152

- Nelson, 19, 105

- - rays, Diagram of, 124

- - Use of, 124 
Condenser, auxiliary, Watson-Conrady, 105, 124

- Zeiss' aplanatic, 106

Condenser, Substage, 102

- Abbe, 107

- - Achromatic, 108

- - Achromatic, 108, 109, 112

_ - and Abbe compared, 111

- - To centre, 118,145

- Choice of, 111

- Comparison of corrected and uncorrected, 111

- Dark ground, 183

- Focal length of, 108

- - To focus, 118,146

- _ - with parallel light, 148

- - for large objects, 109,137

— Leitz' oil immersion, 109, 182

- Low-power illuminator, 107, 109

- N.A. of, 108,121

- Objectives as, 113

- Oil immersion, 109

- - Power of, 109

- Swift's achromatic and paragon, 109

_- Watson's universal and parachromatic, 109

Confusion, Circle of, 64

Connecting cells, Light-tight, 10

Contrast in photomicrography, 161, 225,253

— in prints, 229,257

- Screens, 161

- - for bacteria, 241

- - for insects, 252

Cooling troughs, 149

Copper sulphate ammoniacal solution, 158

Correction Collars for Objectives, 70

Corrosive acetic fixing fluid, 263

Counterstains, 265

Cover-glass reflector, 195

- - Thickness of, 69

_ $\ldots$ and tube length, 70
Critical light, image, illumination, 83,114

- - and even illumination, 83 , 120,123

- - To obtain, 146

Culture tubes, 246

Cycas revoluta, 261

Dark ground condenser, 183

- - illumination, 179

- - - Adjustment for, 186

- - bacteria, for, 245

- - Central stop for, 179

_ - - high powers, for, 182

- - - instantaneous photomicrography, 247

- - - low powers, for, 182

- - Monochromatic light for,

187

- - Objectives for, 187

_ _ - plate cultures for, 247

- - Radiants for, 186

Dark-room lamp, 235

Davis' iris diaphragm, 181

Daylight diffused. (See Sunlight)

Demonstration Eyepiece, 73, 247

Developers, 220

- Concentrated (Azol, Certinal, Rodinal, Victol, 220, 269

- formulæ, 268

- Hydroquinone, 221, 268

- Metolquinol, 221, 268

- Potass bromide in, 221

- Pyro Soda, 220, 269

- Rytol, 220

- Tabloid, 220

Development, 221

- factor, 216

- Factorial, 224

- papers, 228

- Stand, 223

- Tank, 132, 223

- Time, 222

Diaphragm. (See Stops)

- Auxiliary iris or bench iris, 106, 126,148 
Diaphragm, Davis', 181

- lamp, on, 97

- Nosepiece iris, 181

- Substage, 28

Diatoms: Actinoptychus heliopelta, 255

- Amphipleura pellucida, 177, 257

- Arachnoidiscus ehrenberghii, 254

- Development for, 257

- Navicula crabro, 255

- lyra, 255

- - rhomboides, 178

- Photomicrography of, 253

- Pleurosigma angulatum, 256

- Resolution by oblique light, 177

- Surirella gemma, 178, 256

- Test objects, $57,178,254$

- Triceratum favus, 255

Diffraction effects with bacteria, 240

- and restricted N.A., 121

Dispersive power, 49

Dissecting microscope, 139

Dissections, Photomicrography of, 250

Draw tubes, 34

Drying negatives, 225

Dufay colour plates, 210

Duplicating colour photography, 211

Dust on lenses, 144

Dyes for three-colour process, 207, 273

Ehrlich's hæmatoxylin, 261, 265

Ellipsoid enlarger, 129

Embryo chick, 260

Enlargement table, 275

Enlargements compared to highpower photos, 230

Enlarging, 230

- with photomicrographic apparatus, 231

- with reflecting apparatus, 232

Eosin, 266

Equivalent focal length, and lens, 59

Ernemann's microcinematograph, 189

Ether saturator, 95

Exposure, 147, 169
Exposure, diatoms, for, 257

- Errors in, 226, 257

- factors, 171

- - of "M" Screens, 174

- and immersion objectives, 69

— and light source, 173

- and magnification, 173

- and N.A., 172

- notebook, 170

- Over-, 226

- Record of, 171

- Trial-, 169

- Under-, 226

- Unit of, 175

Extension of camera and magnification, 77, 131

- Extra bellows, 9

Eyepiece, Achromatic, 74

- Compensating, 55, 72

- Demonstration, 73, 247

- Designation of, 74

- Holoscopic, 74

- Huyghenian, 72

- objective, and, 74

- Power of, 76

- - limit, 74, 76

- Projection, 72, 152

Factorial development, 224

False images and diffraction, 245

- - and oblique light, 254

Farmer's reducer, 271

Fermentation industries and photomicrography, 243

Ferrotype, plate polish for, 276

Films, 219

Filters, Light. (See Colour Screens

Fine adjustment, $32-34$

- - Lever, 33

- - Prismatic bar, 32

- - Rapidity of, 32

- - Safety, 34

- - testing, 33

- - Substage, 28, 39

Fixing plates, 224

- reagents, 258 
Fixing fluid, Acetic-alcohol, 264

- - Chromo-acetic, 263

- - Corrosive-acetic, 263

- Flemming's, 264

- Hermann's, 263

- - Perenyi's, 263

- Solution, 270

Flare spot, 138

Flasks as condensers, 93

Flats, optical, 155

Flea, 252

Flemming's fixing fluid, 264

Fluorite, 53

Focal length of condenser, 120

- - Equivalent, of objectives, 59

Focal planes, 59

Focus, 146

Focussing gear, 9, 19, 138

- Hooke's handle, 10

- lens, 11

- screen, Card, 10

- Clear glass, 11

- - Ground glass, 10, 11

- stage for metallography, 197

- substage condenser, 146,148

Foot, tripod and horseshoe, 45

Fritillaria root apex, 262

Gaslight papers, 228, 257

Gelatine colour screens, 155

- - To make, 156

Geometric slide camera, 19

Glass crown and flint, 50

- Jena, 53

- Optical, 53

- Refractive indices of typical, 276

Glazing prints, 230

Glycerin as inmersion fluid, 67

Gnat, 252

Goldberg, Dr. E., on "Sharpness of a Plate," 216

Gordon's camera, 21

Grain of plates, 216

Green screen for definition, 61, 254

Ground glass for even illumination, 122,133
Hageh mercury vapour lamp, 93

Heidenhain's iron haematoxylin, 265

Halation, 217

Hardening negatives, 225

- solutions, 271

Heliostat, 84

Hermann's fixing fluid, 263

Holoscopic eyepiece, 74

Holostigmat, 79

Homogeneous immersion, 67

Horizontal camera, 2. (See Camera)

Horseshoe foot, 45

Huyghenian eyepiece, 72

Hydra lantern plates, 234

- panchromatic plates, 214

Hydroquinone developer, 221, 257, 268

Hypo and acid hypo fixing baths, 270

- Eliminators, 225, 270

Ilford Panchromatic plates, 214

Illuminants. (See Radiants)

Illumination, 145

- auxiliary condenser, with, 147

- convergent light, $124,136,150$

- critical light, 114, 146

- Even, 120123

- Management of, 124

- Parallel, 125

- planars, for, 136

- reflectors, by, 128

- small solid objects, for, 128

- two condensers, with, 125,134

Imbedding in paraffin wax, 259

Immersion fluids, 67

- - and penetration, 65

- objectives, 67

- of objects for photomicrography, 65,250

Incandescent Electric light, 91

- gas, 97

Initial magnifying power, 78

Insects, Camera for, 130

- Photomicrography of, 251

- Preparation of, 252. 
Instantaneous photomicrography, 247

Intensification, 227

Intensifying solutions, 271

Iodine green, 266

Japanese colours, 235

Japine platinotype, 230

Jena glass, 53

Karyokinesis, 262

Klebs-Löffler bacillus, 242

Kingsford trough, 13, 160

Köhler condensers, 105, 134

Lamp casing, 13, 100

- Dark-room, 235

- stands, 100

Lamps. (See Radiants)

Lantern, Optical, 8

- to enclose radiant, 13,100

- slides, 233

- - Binding, 235

- Colouring, 235

- - Improvement of, 234

- - by reduction, 234

- - Toning, 235

Leg of Honey Bee, Photograph of, 251

Leitz's camera for Insects, 130

- condenser, oil immersion, 109, 182

- dark ground illuminator, 183

- Liliput are, 7, 88

- mechanical stage, 27

- micro-metallograph, 201

- microscope, "A," 41

- - students', 44

- photomicrographic apparatus, 18

Lenses, achromatic and apoehromatic, 52

- Batteries of, 81

- Correction of, 49

- for enlarging, 233

- for low-power photomicrography, 79,130

- Micro-anastigmat, 79
Lenses, Telephoto, 139

Leto toners, 235

Leybold's trough, 160

Lieberkuhn, 192

Light, Critical, 114

- tilters. (See Colour Screens)

- - for Mercury vapour lamps, 94

- tight connection for camera and microscope, 10, 138

- Wave length units, 158

Liliput arc, 7,88

Limelight, 95

- blow through and mixture jets, 95

- ether saturator, 95

- injector jet, 96

Liquid light filters, $15 \pi$

Living micro-organisms, Mounting of, 243

- - Photomicrography of, 243, 248

- - Prevention of movement of, 243

Löffler's stain, 266

Louse, Photograph of, 252

Low-power photomicrography, Adjustments for, 132, 138

Lumière's Autochromes, 210, 274

- extra sensitising solution, 213

"M" sereens and plates. (See Colour sereens and plates)

Magnification, 75

- and camera extension, 77, 131

- - exposure, 173

- - eyepiece, 77

- - focal length, 75

- - tube length, 77

- Initial, of objective, 76,78

- of telephoto lens, 140

- To determine, 75

Marten's metallurgical microscope, 199

Mayer's carmalum, 261, 264

Measuring tape for projection eyepieces, 152 
Mechanical stage, 27. (Sce Stage) Medical Practice and photomicrography, 239

Mees, Dr. C. E. K., on " Resolving Power of Plates," 216

Mercury vapour lamp, 92, 93

- - L Light filters for, 94

- - Quartz tubes for, 93

- - Spectrum of, 94

"Meta" Spirit vapour lamp, 98

Metal surfaces, photographs of, 194

Metallography, 194

- Critical illumination for, 196

- Vertical illuminator for, 195

Metallographic microscope, focussing stage, 197

- Marten's, 199

- Ordinary microscope adapted for, 199

- - Rosenhain's, 198

- Substage used as stage, 200

Methylene blue, Spectrum of, 167

Metol-quinol developer, 221, 268

Micro-anastigmat, 79

- Illumination for, 136

- Magnification by, 80

- N.A. of, 80

Micro-metallographs, 201

Micro-metallography, 194

Micron, 158, 275

Micro-organisms, living, Photography of, 243

- - Mounting, 243

Micro-planar, 79

Microscope, 25

- Baker's, 41

- Bausch and Lomb's, 39, 43

- Beck's, 45, 148, 198

- Black finish, 26

- Coarse adjustment, 31

- Cost of, 35, 36

- Dissecting, 139

- Edinburgh student's, 43

- English and Continental compared, 44

- Experiments with, 116
Microscope, Fine adjustment of, 32-34

- Foot, tripod and horseshoe, 45

- Imperial, 148

- Large, 37

- Leitz, "A.," 41

- - Students', 44

- Limb, 26

- London, 45

- Marten's, 199

- Medium, 43

- Metallurgical, 198

- Mirror, 31

- Non-inclinable, 44

- Premier, 37

- Rigidity of, 26

- R.M.S., 41

- Rosenhain, 198

- Ross', 45.

- Spencer, 39

- Stability of, 26, 45

- Stage, Mechanical, 27. (See Stage)

- - Adaptable, 27

- - Rotating, 27

- Standard, 45

- Students', 43

- Substage, 28. (See Substage)

- Swift's, 37

- tube diameter, 35

- - length, 34

- Twin lens, 194

- Van Heurck's, 39

- Watson's, 39, 43

- with inverted objective, 202

- Zeiss' No. 1, 41

Micro-sumnar, 79

Mirror, use of, 117

- to centre, 117

Monochromatic light, 154

- - for dark ground illumination, 187

- objectives, 52

Moulds, Plotography of living, 243

Mounting media, Refractive indices of, 64,276

Mounting sections, 260 
N.A. (See Numerical Aperture)

Napthalene mono-bromide immersion fluid, 67

Narcotic for living organisms, 249

- fluid, 268

Natural History objects, Opaque, 191

Navicula crabro, 255

- lyra, 255

- rhomboides, 178

Negatives, Contrast in, 225

- Improvement of, 226

Nelson's condenser, 105

- dark ground condenser, 183

- definition of critical light, 114

Nernst lamp, 19, 91

- - Zeiss', 92

Nosepiece, Centring, 29

- Diaphragm, 181

- Rotating, 30

- Sliding objective changer, 30

Notebook for exposures, 170

Nurnerical Aperture, Abbe's definition of, 60

- - bacteria, for, 241

- - condensers, of, 103

- - and depth of focus, 64,122

- - diatoms, for, 254

- - diffraction, and, 121, 255

- - dry lenses, 255

- _ "f" ratio, 79

— - flat field, and, 65,255

- - living micro-organisms, for, 244

- - maximum useful, 121

- - objects various, for, 121

- - oil immersion objectives, 67

- - penetration and, $64,122,255$

- - powers, for various, 66

- - resolution, $60,67,255$

- - spherical error, and, 50

- - working distance, and, 66

Objectives, Achromatic, 52

- Apochromatic, 53

- - Brilliance of, 55

- - compared to Achromats, 56
Objectives, Achromatic, N.A. of, 55

- - Round field of, 55

- Superiority of, 55

- - Under-correction of, 55

- Brightness of image of, 63

- Changers for, 30,31

- Combination of, with eyepieces, 74

- condensers, as, 113

- corrected for photography, 52

- Correction Collars for, 70

- cover-glass thickness, and, 69

- Definition of, 65

- Equivalent focal length of, 59

- Flatness of field of, 65

- Fluorite, 54

- Focal length and power of, 59

- Focal planes of, 59

- Holoscopic, 54

- Improved achromatic, 54

- Initial magnifications of, 59,76 , 78

- - - Table of, 81

- Mono-chromatic, 52

- Numerical Aperture of, 60

- Oil immersion (q.v.), 67

- Penetration of $(q \cdot v \cdot), 63$

- Resolving power of $(q . v),$.

- Semi-a pochromatic, 54

- Sets of, 58,80

- Short, for metallography, 196

- Testing, 56

- Under-correction of high-power, 54

- Working distance of (q.v.), 65

Objects, Preliminary examination of, 143

Oblique illumination, 176

- - Amphipleura and, 177, 257

- - Condenser for, 179

- Continental Substage, and, 177

- - false images, and, 176, 254

- - resolution, and, 176,178

- - for Surirella, 256

- - Stops for, 177

Oil immersion condensers, 109 
Oil immersion ohjectives, 67

- - and brightuess of image, 69

69

69

- - cover-glass correction, 70

- - Exposure with, 69

Oil lamps, 99

Opaque objects, Photography of, 191

Optical bench, 2

- - Beck's, 148

- - Geometric slide, 19

- - made of bicycle tubing, 199

- Zeiss' large, 150

- Zeiss' triangular bar, 200

- flats, Coloured, 155

- lantern, 8

- tube length, 76

Orange G., 266

Orthochromatic plates, 166,215

Paget colour plates, 210

- duplicating colour, 211

Panchromatic plates, 166, 214

Parabolic reflector, 192, 199

Paraboloid, Wenham, 179

- Watson's, 184

- Zeiss', 184

Parachromatic condenser, 109

Paraffin wax for imbedding, 259

Paragon condenser, 109

- plates, 220

Paralleliser, Adjustment of, 147

Parallel light, Illumination with, 147

Parasites, Photography of, 252

Penetration, 63

- immersion of object, and, 250

- iris diaphragm, and, 64

- N.A., and, 64

- refractive index of medium, and, 64

Perenyi's fluid, 263

Perfectol lamp, 98

Persulphate reducer, 271

Petrol vapour lamp, 98

Petrology, 189
Photomicrography, Applications of, 238

- evidence, as, 2

- adulteration, and, 238

- Medical practice, and, 239

- research, and, 2

(See also under individual subjects)

Photomicrographic apparatus, Sets of, 277

Picric Acid, 266

Planars, 79

- Illumination for, 136

Plant sections, 257

Plate Cultures, 246

Plates, Autochrome, 210

- Backed, 219

- Chromo-Isolar, 220

- Colour sensitive, 165

- Dufay, 210

- Gem Tricol, 214

- Grain of, 216

- Hydra, 214

- Ilford panchromatic, 214

- Lippmann, 216

- Paget Colour, 210, 212

- Panchromatic, 214

- Panchromatic, 166, 214

- Paragon, 220

- Resolving power of, 216

- Sanger-Sliepherd, 214

- Speeds, 215

- Wratten and Wainwright's " $M$ " and Panchromatic, 214

Platinotype, 22y

- developer, 272

Plentosigma angulatum, 256

Polarised light, 189

Pole paper, 89

Polish for ferrotype plates, 275

Polyzoa, 249

Pond life, 247

P.O.P., 228

Powell and Lealand Apochromatic Condenser, 110

- - Objective, 67

Premier microscope, 37 
Printing processes, 227

- - for contrast, 229, 257

Prints, in colour, 203

Prism vertical illuminator, 196

- attachmeut for vertical illuminator, 200

Projection eyepieces, 72

- - adjusting tape for, 73, 152

Pyro-soda developer, 220, 269

Quartz objectives for ultra-violet light, 63

- tubes for Mercury vapour lamp, 93

Radiants, Acetylene (q.v.), 96

- $\operatorname{Arc}(q . v),$.

- candle powers, 101

- Casing for, 13,100

- Colour of, 164

- Comparison of, 100

- and Critical light, 122

- Electric, 85

- and exposure, 173

- Heat of, 84

- incandescent electric, 91

- - gas, 97

- Lantern to enclose, 13

- - optical, 8

- Lilliput arc, 7, 88

- Limelight (q.v.), 95

- Mercury vapour (q.v.), 92

- Meta, 98

- Nernst (q.v.), 19, 91

- Oil, 99

- - and Critical light, 100

- Petrol vapour, 98

- Selection of, 100

- Spectra of, 84

- Spirit vapour, 98

- Stands for, 100

- Structure of, 84

- Suitability of, 83

Ramsden circle, 119

Rectilineal lenses, 130

Reducers, Farmer's and persulphate, 271
Reduction of negatives, 227

Reflecting condensers for dark ground, 183

Reflections in tube, to eliminate, 139 Reflector for low-power photo, 128

- for enlarging, 232

Reflex camera, 5,13

Refractive indices, 49, 276

- - of mounting media, 64,276

Reichert's micro-metallograph, 201

Resolution and Exposure, 217

- and Grain of plates, 216

Resolving power of objectives, 61

— — dry and oil objectives, 69

- - Limit of, 62

- - and N.A., 60

- - and ultra-violet light, 63,176

- - and wave length, 61

Rodinal, 269

Root sections, 258

Rosenhain's metallurgical microscope, 198

Ross mechanical stage, 27

- microscopes, 45

Rotifers, 249

- narcotization and mounting, 249

Saddlc stands, 13

Safelight for Panchromatic plates, 236

- for testing Panchromatic plates, 237

Safranin, 265

Sanger-Shepherd colour screens, 153

- - - positive, 206

- - films, 206

- - three-colour process and filters, 205

Screen-plate colour photography, 209,273

Screens. (See Colour screens)

- for colour photomicrography, 204, 208,210

- for daylight and artificial lights, 204

- Focussing, 10 
Screens for various stains, 267

- White card, for light adjustment, $10,19,134$

Secondary speetrum, 51

- - structure in cliatoms, 255

Section eutting, mounting, staining, 258-260

- double staining, 261

Semi-apochromats, 52

Semi-glossy papers, 229

Sensitising solution for colour plates, 270

- - for transparencies, 273

Shells, photography of, 251

Sliding objective ehanger, 31

Solid objects, photo of, 249

Solutions for light filter's, 158

Specimens in bottles, 250

Spectacle lens condensers, 107

Spectra, Absorption, 151

- - Diagram of, 159

- of Radiants, 84

Spectrum of Mercury vapour lamp, 94

Spencer microscope, 39

Spherical aberration, 47

Spider, 250

Spirillum, 242

Spirit vapour lamp, 98

Spirochaeta pallida, 245

- plicatilis, 242

Spores staining, 267

Spot lens, 179

Spotting negatives, 226

Stage (microscope), adaptable, 27

- complete, 28

- mechanical, $2 \pi$

- rotating, 27

- Ross micrometric, 27

- Zeiss photomierographic, 41

Staining bacteria, 242

- in bulk, 261

- Counter, 261

- sections, 260

Stains, Aniline blue, 265

- for bacteria, 266

- Borax-carmine, 264
Stains, Carbol-fuchsine, 266

- Carmalum, 261, 264

- Eosin, 266

- Gram's, 266

- Hærnatoxylin, Ehrlich's, 261, 265

- - Heidenhain's, 262, 265

- Iodine green, 266

- Löffler's methylene blue, 266

- Orange G., 266

- Picric acid, 266

- Safranin, 265,

Stand development, 226

- Microscope, 26

- Lamp, 100

Stems, sections of, 258

Stereoscopic photomicrography, 192

- Centring nosepiece for, 194,

- - prints, 194

- - Stops for, 194

- - twin lens microscope, 194

- - two exposures, 194

Stops, conical, for high-power objectives, 181

- cylindrical, for low-power objectives, 192

- Davis, 181,

- for oblique illumination, 177

- for stereoscopy, 194

- Traviss' expanding, 180

-- Wheel, 179, 180

Substage, 28

- centring, 29

- condenser. (See Condenser)

- English and Continental, 44

- fine adjustment, 28

- Fixed sleeve, 28

- iris, 28

- - Adjustment of, 146

- Lateral screw, 28

- Rack and pinion, 28

- ring as object carrier, 200

Summars, 79

Sunlight as illuminant, 84

Surirella gemma, 178

Suspension of camera, 24

Swift's achromatic condensers, 109 
Swift's apochromatic condensers, 110

- Premier microscope, 37

Table for camera and optical bench, 21

Tabloid developers, 220

Tank development, 132, 223

Tape for projection eyepieces, 152

Telephoto lens, 139

- - extension with, 140

Tenth metre, 158

Test objects, 57

- - Diatoms as, 57,178

- - Amphipleura, 257

- - Navicula, 255

- - Pleurosigma, 256

Thames colour plate, 211

Three-colour photography, 204

- - - for bacteria, 241

— - Dyes for, 207, 273

- - Exposures for, 205

- - - Filters for, 205

- - - positives, 206

Time development, 222

Toning lantern slides, 235

- blue solution for transparencies, 273

- P.O.P., 272

Transmission of filters, 154,159

Transparencies in colour, 203

Transparent objects, low-power, 133

Traviss' expanding stop, 180

Triceratium favus, 255

Tricolour process. (See Three-colour process)

Tripod, foot, 26, 45

Troughs for liquid filters, 160

- Kingsford, 13

'Tube diameter, 35

- Wide, 139

- length, 34, 70

- - and cover-glass thickness, 70

- - and magnification, 77

- Mechanical, 70

- Optical, 76

Turbidity factor of plates, 216
Two-colour process, 208

- - - for bacteria, 241

Ultra-microscope, 185

Ultra-violet light for photomicrography, 63

- - and resolution, 63,176

Universal cameras, 17

- condenser, 109

Van Heurck camera, 15

- - microscope, 39

Verniers on stage, 27,143

Vertical cameras. (See Cameras)

- - for solid objects, 250

- illuminators, 195, 196

- - Prism attachment, for, 200

Vibration, prevention of, 21, 23

Victol, 269

Violet light and resolution, 61

Wales microscope stand, 37

Water immersion objective, 67

Watson's camera, 11

- Conrady condenser, 13, 105, 124

- Holoscopic eyepieces, 74

- microscopes, 39,43

- paraboloid, 184

- substage condensers, 109

Wave length units, 158

Weights and measures, English and metric, 274

Wenlaam's paraboloid, 179

Westminster enclosed are, 90

Wheel stop, 180

White light, disadvantages of, 153

Wide angle cone of light and condensers, 109

- $-\ldots$ and objectives, 67

- - - - for bacteria, 241

Wids tube, 139

Working aperture, 111

- distance, 65

- - and N.A., 65

Workroom, 22

Wratten's colour screens, 156, 267 
Wratten's panchromatic plates, Zeiss' mechanical stage for photo214

- three-colour filters, 205

Yeast, living, 243

Zeiss' 5 ampère arc, 87

- camera, large, $21,150,232$

- - vertical and horizontal, 19, 134

- condensers, 105

- aplanatic, 106, 136

- - Köhler, 134, 150 micrography, 41

- mercury vapour lamp, 93

- microscope No. 1, 41

- Nernst lamp, 92

- optical bench, large, 150

- - triangular bar, 200

- paraboloid, 184

- Planar, 79

- sliding Objective changer, 31

- tape for projection eyepieces, 73 , 152

— vertical illuminator, 196

\section{THE END}





\section{FOURTEEN DAY USE}

\section{RETURN TO DESK FROM WHICH BORROWED}

\section{Biology Litbrary}

This book is due on the last date stamped below, or on the date to which renewed.

Renewed books are subject to immediate recall.

\section{. DEC 31955}

\section{JAN 261956}

JUN 4 '57

JUN $4 \quad 1957$

OCr 251958 $260 c^{\prime} 5806$

OCT 211961

COT 21 1901

JUN 41963

AUG 301963

JUN 301966

197

OEC 10 1971

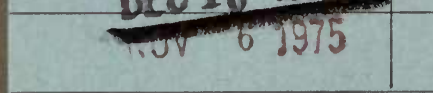




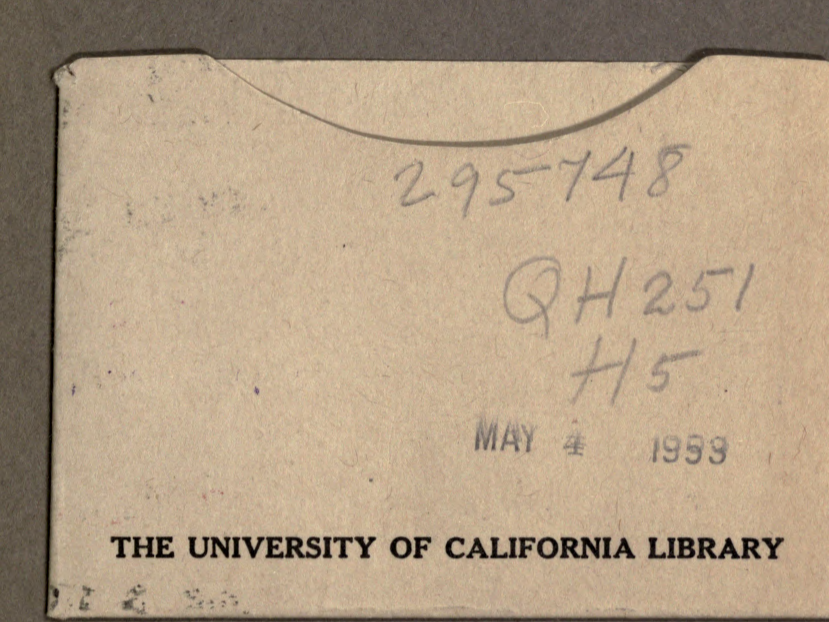


